\title{
RAPID AND PORTABLE ELECTRICAL LYSIS AND ELECTROPORATION DEVICE
}

by

Bashir Iqbal Morshed, B.Sc., M.A.Sc.

A dissertation submitted to the Graduate Studies and Research

in partial fulfillment of the requirements for the degree of

\section{Doctor of Philosophy}

in

Electrical and Computer Engineering

Ottawa-Carleton Institute for Electrical and Computer Engineering

Department of Electronics

Carleton University

and

Medical Devices Centre

University of Ottawa Heart Institute

May 2010

(C) May 2010 


$\begin{array}{ll}\begin{array}{l}\text { Library and Archives } \\ \text { Canada }\end{array} & \begin{array}{l}\text { Bibliothèque et } \\ \text { Archives Canada }\end{array} \\ \begin{array}{l}\text { Published Heritage } \\ \text { Branch }\end{array} & \begin{array}{l}\text { Direction du } \\ \text { Patrimoine de l'édition }\end{array} \\ \begin{array}{l}\text { 395 Wellington Street } \\ \text { Ottawa ON K1A ON4 } \\ \text { Canada }\end{array} & \begin{array}{l}395, \text { rue Wellington } \\ \text { Ottawa ON K1A ON4 } \\ \text { Canada }\end{array}\end{array}$

Your file Votre reférence

ISBN: 978-0-494-67894-7

Ourfile Notre référence

ISBN: 978-0-494-67894-7

NOTICE:

AVIS:

The author has granted a nonexclusive license allowing Library and Archives Canada to reproduce, publish, archive, preserve, conserve, communicate to the public by telecommunication or on the Internet, loan, distribute and sell theses worldwide, for commercial or noncommercial purposes, in microform, paper, electronic and/or any other formats.

The author retains copyright ownership and moral rights in this thesis. Neither the thesis nor substantial extracts from it may be printed or otherwise reproduced without the author's permission.

\begin{abstract}
L'auteur a accordé une licence non exclusive permettant à la Bibliothèque et Archives Canada de reproduire, publier, archiver, sauvegarder, conserver, transmettre au public par télécommunication ou par l'Internet, prêter, distribuer et vendre des thèses partout dans le monde, à des fins commerciales ou autres, sur support microforme, papier, électronique et/ou autres formats.
\end{abstract}

L'auteur conserve la propriété du droit d'auteur et des droits moraux qui protège cette thèse. $\mathrm{Ni}$ la thèse ni des extraits substantiels de celle-ci ne doivent être imprimés ou autrement reproduits sans son autorisation.
In compliance with the Canadian Privacy Act some supporting forms may have been removed from this thesis.

While these forms may be included in the document page count, their removal does not represent any loss of content from the thesis.
Conformément à la loi canadienne sur la protection de la vie privée, quelques formulaires secondaires ont été enlevés de cette thèse.

Bien que ces formulaires aient inclus dans la pagination, il n'y aura aucun contenu manquant. 


\begin{abstract}
The Issue: Cell lysis (rupture of cellular membranes) is one of the most critical step in accessing Deoxyribonucleic Acid (DNA) for genetic analysis. Current cell lysis methods applied to DNA extraction is primarily chemical agent based. Such method is slow, laborious, and requires human intervention. To reduce these limitations, a high electric field based rapid electrical lysis method integrate-able on a portable micro-device was investigated.

Purpose: To innovate a rapid and portable cell lysis device by applying electrical lysis method towards extraction of DNA from non-invasively collected human cells, and study relevant phenomena.

Method: After review of recent scientific articles on electrical lysis and related phenomenon as electroporation, electrical lysis and electroporation were studied though development of three devices iteratively. Device designs, modelling, simulations, experiments and analysis results are presented in this thesis. A novel membrane pore model to represent dynamics of electroporation in the current electrical circuit cell model inside an electric field was also developed and presented.

Results: (1) The initial device demonstrated the cell lysis of non-invasive human cells with a high electric field, but required a high voltage supply (750 to $900 \mathrm{~V})$. (2) The intermediate device produced limited success due to issues like fluidic encapsulation, bubble formation and cell confinement. (3) The final device demonstrated ability of cell lysis and release of DNA from nucleus of cell samples. (4) Cell lysis to release DNA required an electrical excitation of $20 \mathrm{~V}$ for $5 \mathrm{~s}$ duration. (5) Electroporation phenomenon was observed by applying pulses of 5 to $20 \mathrm{~V}$ magnitude and $10 \mu$ s to $100 \mathrm{~ms}$ durations. (6) The proposed pore model simulation results were validated by experimental
\end{abstract}


results. (7) Electroporation quantification terms were defined and validated: (a) Degree of Electroporation, (b) Electroporation Fluid Exchange Parameter (EFEP).

Conclusions: We have demonstrated applicability of electrical lysis for DNA extraction from human cells. This research resulted a novel, small, rapid, portable, battery operable micro-device that is capable of performing both electrical lysis and electroporation effectively, as well as a pore model representing electroporation dynamics. 


\section{ACKNOWLEDGMENTS}

I am indebt to Dr. Tofy Mussivand for problem identification, concept development, and experimental guidelines, and Dr. Maitham Shams for the device designs, manufacturing, modelling, analysis and experiments to proceed through this research. I want to extend my thanks to the staff of Medical Devices Centre at the University of Ottawa Heart Institute and the Department of Electronics at Carleton University for all their helps and suggestions, especially to John Szalas of Medical Devices Centre, Rob Vandusen of Department of Electronics, and Dr. M. Emdadul Haque of Cellular and Molecular Medicine, University of Ottawa. I thankfully recognize the funding provided by Ontario Ministry of Training and Education, Indira Gandhi Fellowship, Graduate Studies of Carleton University, the Department of Electronics, and Medical Devices Centre. I also like to acknowledge significant contributions of my parents, wife and charming daughter for all inspirations, support and encouragement to move forward in some times of despair. 


\section{Contents}

Table of Contents vi

List of Figures $\quad$ xiii

List of Abbreviations and Acronyms xxxii

Notations and Symbols $\quad \operatorname{xxxv}$

1 Introduction $\quad 1$

1.1 Overall Project of a DNA Detection Device. . . . . . . . . . . 1

1.1.1 Conventional DNA detection process . . . . . . . . . 2

1.1.2 Advancements in DNA detection process . . . . . . . . . 4

1.1.3 Limitations of current DNA detection process . . . . . . . 4

1.1.4 DNA detection device project $\ldots \ldots \ldots \ldots$

1.1.5 Rationale of this research $\ldots \ldots \ldots \ldots$

1.2 Overview of This Research Work . . . . . . . . . . . 7

1.2 .1 Objective and scope $\ldots \ldots \ldots \ldots \ldots \ldots$

1.2.2 Some relevant terms and definitions . . . . . . . . . . 7

1.2 .3 Current cell lysis methods . . . . . . . . . . . . . . 9 9

1.2.4 Proposed cell lysis method . . . . . . . . . . . . . 9

1.2.5 Challenges and technology gap . . . . . . . . . . 10

1.3 Research Approach . . . . . . . . . . . . . . . . . 11

1.3.1 Concept development . . . . . . . . . . . . . . . . 11 
1.3.2 Choice of fabrication technology . . . . . . . . . . 11

1.3.3 Device design and modelling ............. 15

1.3.4 Testing and evaluation ............... 15

1.3.5 Data analysis .................. 16

1.4 Summary of Results . . . . . . . . . . . . . . . . 16

1.4.1 Key findings and important results . . . . . . . . . . 16

1.4.2 Distinguishing attributes of the final device. . . . . . . . 18

1.4.3 Contributions .................. 20

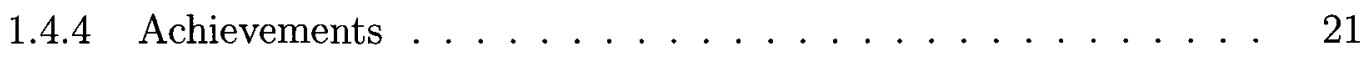

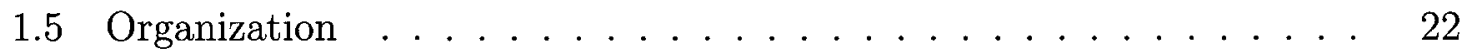

2 Literature Review 23

2.1 Cell Biology . . . . . . . . . . . . . . . . 23

2.1.1 Composition of the cell membrane . . . . . . . . . 23

2.1.2 Nucleus of a cell . . . . . . . . . . . . . . 24

2.2 Dynamics of Electroporation and Electrical Lysis . . . . . . . . . . 26

2.2.1 Development of transmembrane potential and formation of pores 26

2.2.2 Reversible electroporation phenomenon . . . . . . . . . 27

2.2.3 Irreversible electrical lysis phenomenon . . . . . . . . . . 28

2.3 Studies of electroporation and electrical lysis . . . . . . . . . . . 28

2.3.1 Recent advancements in electroporation . . . . . . . . 29

2.3.2 Recent advancements in electrical lysis . . . . . . . . . 31

2.3.3 Chronological list of major findings . . . . . . . . . . 33

2.4 Modelling of Electroporation . . . . . . . . . . . . . 33

2.4.1 Mathematical model ................ 38

2.4.2 Electric circuit model . . . . . . . . . . . . . . 41

2.4.3 Molecular dynamics approach . . . . . . . . . . 43

2.4 .4 Numerical modelling . . . . . . . . . . . . 44

2.5 Applications of Electroporation and Electrical Lysis . . . . . . . . . 45 
2.5.1 Electroporation applications ............. 46

2.5.2 Electrical lysis applications . . . . . . . . . . . . 46

2.6 Other Effects of a High Electric Field on Cells . . . . . . . . . . . . 47

2.6.1 Cellular spin resonance . . . . . . . . . . . . 47

2.6.2 Electrofusion .................. 47

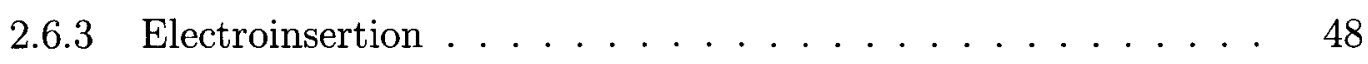

2.6.4 Electroactivation ................. 48

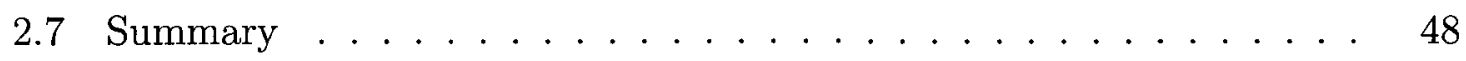

3 Device Designs $\quad 50$

3.1 The Initial Device . . . . . . . . . . . . . . 51

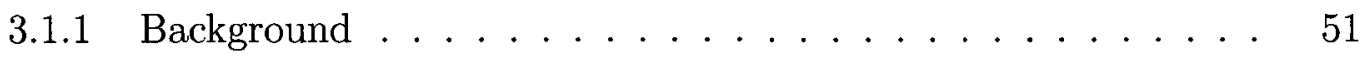

3.1 .2 Device Design ...................... 51

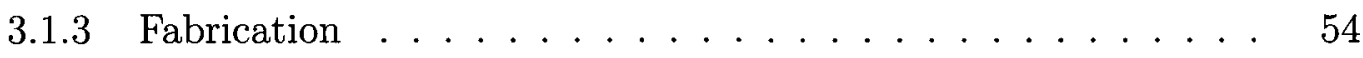

3.2 The Intermediate Device . . . . . . . . . . . . . 56

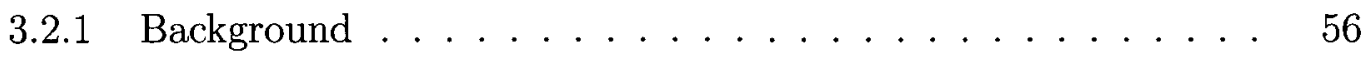

3.2.2 Design and fabrication of the intermediate device . . . . . 56

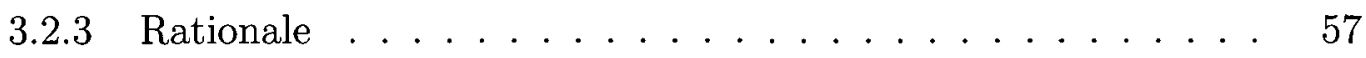

3.2.4 Important design features . . . . . . . . . . . 57

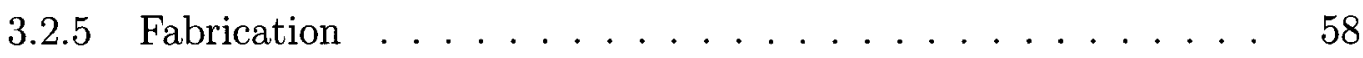

3.3 The Final Device . . . . . . . . . . . . . . . . . . 61

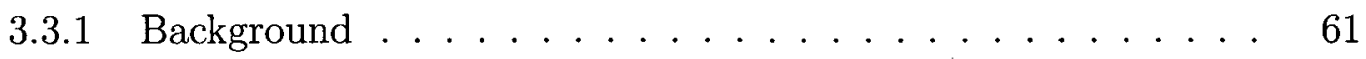

3.3.2 Design of the final device . . . . . . . . . . . 61

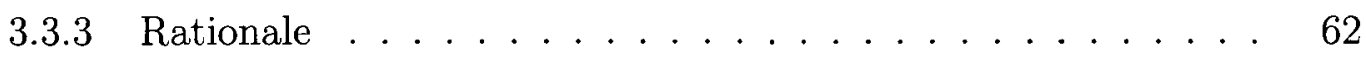

3.3 .4 Important design features . . . . . . . . . . . . 62

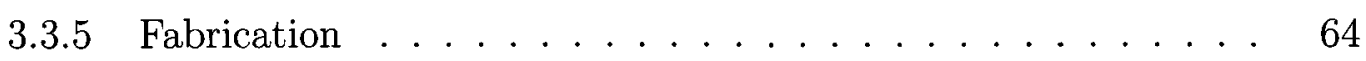

3.4 Discussion on the Novelty of the Final Device . . . . . . . . . . . . 64

3.5 Summary ............................. 68 
4 Simulation Results $\quad 69$

4.1 Simulation of Electric Field Inside Microchannels . . . . . . . . . . . 69

4.1.1 Background and rationale of the analysis . . . . . . . . 71

4.1.2 Ideal model of a microchannel . . . . . . . . . . . . 72

4.1.3 Analytical expression for the ideal model . . . . . . . . . 72

4.1.4 Simulation of an ideal microchannel model . . . . . . . . 74

4.1.5 Model of a microchannel with external electrodes in reservoirs $\quad 79$

4.1.6 Simulations of the microchannel with external electrodes . . . 79

4.1.7 Extended analytical model to reduce estimation errors . . . . 82

4.1.8 Simulation of the initial device. . . . . . . . . . . 84

4.1.9 Electric field simulation of the final device . . . . . . . . 87

4.2 Thermal Simulation . . . . . . . . . . . . . . . . . . . . 89

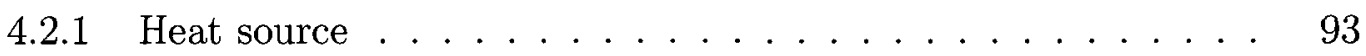

4.2.2 Modelling of the device . . . . . . . . . . . . 93

4.2.3 Settings for sub-domains and boundary conditions . . . . . . 95

4.2.4 Meshing and simulation of the model . . . . . . . . . . . 95

5 Experimental Results $\quad 108$

5.1 The Initial Device . . . . . . . . . . . . . . . . . . . 108

5.1.1 Chemical reagents................. 108

5.1.2 Equipment and setup ............... 109

5.1.3 Sample collection and preparation ........... 110

5.1.4 Brief description of the method ............. 111

5.1.5 Measurement of impedance of microchannels . . . . . . . . 112

5.1.6 Loading of buffer fluids in microchannels . . . . . . . . . . . 114

5.1.7 Moving cells through the microchannels . . . . . . . . . 117

5.1.8 Electrical lysis with a high electric field . . . . . . . . . 118

5.1.9 Summary and discussion ............... 124

5.2 The Intermediate Device . . . . . . . . . . . . . . 126 


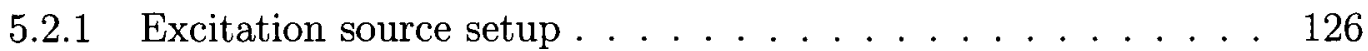

$5.2 .2 \quad$ Hardware programming $\ldots \ldots \ldots \ldots \ldots$

5.2 .3 Drive circuitry for excitation . . . . . . . . . . . . . . . 129

5.2 .4 Fluidic encapsulation . . . . . . . . . . . . . 130

5.2.5 Bubble formation and obstruction of visibility . . . . . . 135

5.2.6 Experimental results of electric pulse excitation $\ldots \ldots \ldots$

5.2 .7 Unresolved issues . . . . . . . . . . . . . . . . . . . . 139

5.2 .8 Discussion . . . . . . . . . . . . . . . . . . . . . 142

5.3 The Final Device . . . . . . . . . . . . . . . . . . . . . 142

5.3.1 Measurement of impedances . . . . . . . . . . . . . . . 143

5.3 .2 Post-processing of the device . . . . . . . . . . . . 145

5.3 .3 Staining of freely flowing cells . . . . . . . . . . . . 145

5.3 .4 Chemicals and equipment $\ldots \ldots \ldots \ldots \ldots$

5.3 .5 Experimental procedure . . . . . . . . . . . . . . . . 149

5.3 .6 Image processing $\ldots \ldots \ldots \ldots \ldots$

5.3 .7 Electroporation experiments . . . . . . . . . . . . . 159

$5.3 .8 \quad$ Electrical lysis experiments . . . . . . . . . . . . . . . . 169

5.3 .9 Reusability of the device . . . . . . . . . . . 175

5.4 Discussion . . . . . . . . . . . . . . . . . . . . . . . . . 179

5.5 Summary . . . . . . . . . . . . . . . . . 179

6 Analysis 182

6.1 Illustrative Diagrams to Represent Electroporation and Electrical Lysis Dynamics . . . . . . . . . . . . . . . . . . 182

6.2 Improvement to the Current Electric Circuit Model of Cell Membrane 187

6.2.1 Current electric circuit model of cell membrane . . . . . . 187

6.2.2 Model parameter extraction with a cylindrical model . . . . 189

6.2 .3 Modelling of pore resistance . . . . . . . . . . . . . . . 192

6.2 .4 Simulation results . . . . . . . . . . . . . . . . . . 197 
6.3 Degree of Electroporation and Electroporation Fluid Exchange Param-

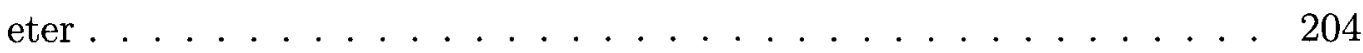

6.4 Discussion . . . . . . . . . . . . . . . . 211

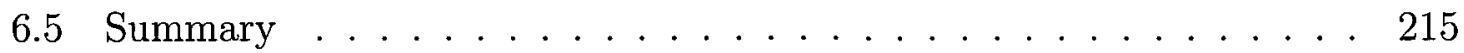

$\begin{array}{lll}7 \text { Conclusions and Future Directions } & 217\end{array}$

7.1 Key Results . . . . . . . . . . . . . . . 217

7.2 Comparison with other devices . . . . . . . . . . . . . . . 219

7.3 Future research directions . . . . . . . . . . . . . . 221

A Device Designs $\quad 222$

A.1 The Initial Device . . . . . . . . . . . . . . . . . . . 222

A.2 The Intermediate Device . . . . . . . . . . . . . . . . . 224

A.3 The Final Device . . . . . . . . . . . . . . . . . . 228

$\begin{array}{ll}\text { B Protocols } & 231\end{array}$

B.1 Developed protocol for fluidic encapsulation . . . . . . . . . . . . 231

B.2 Developed protocol for staining free-flowing cells . . . . . . . . . . . . 233

B.3 Protocol for fixing nanoports on top of fluid ports . . . . . . . . . . . 234

B.4 Protocol for fixing metal electrodes through electrode ports . . . . . . 235

B.5 Developed protocol for reconnecting electrodes . . . . . . . . . . . 237

B.6 Developed protocol for fixing the device on a clean glass surface . . . 237

B.7 Developed protocol for conditioning (wetting) of a microfluidic device 239

B.8 Developed protocol to repair a short circuited integrated electrode . . 239

C Software Codes $\quad 241$

C.1 Script to automate modelling, simulation and data extraction of MFD 241

C.2 Verilog program written for pulse generation of the FPGA board . . . 246

C.3 Visual Basic code listing to extract pixel color info for image analysis 253

C.4 Matlab code to analyze pixel data information and to create plots . . 259 
C.5 Comsol mesh parameters . . . . . . . . . . . . . . . . 261 


\section{List of Figures}

1.1 Steps involved in current DNA detection procedure. . . . . . . . . 3

2.1 (a) Chemical composition of a DMPC molecule - a phospholipid molecule extensively found in cell membranes. (b) Symbolic representation of a phospholipid molecule. (c) A schematic representation of a segment of a cell membrane composed of phospholipid molecules arranged in a BLM formation. . . . . . . . . . . . . . . . . . .

2.2 Model of a cell exposed to an external electric field showing parameters related to the mathematical model. . . . . . . . . . . . . . .

2.3 Equivalent electrical circuit model of a biological cell (with a nucleus) in a suspension medium exposed to an external electric field. . . . . .

3.1 Top view and cross-sectional view of the design template showing the predefined fluidic reservoirs locations and the dimension of the device and reservoirs [Adapted from Protolyne manual]. . . . . . . . . .

3.2 A sketch and a 3D model showing a microchannel structure design in details. Legends: 17- Top glass slide, 18- Bottom glass slide, 19Reservoir, 20- External electrode, 21- Etch pad, 22- Microchannel. .

3.3 A photograph of the fabricated microfluidic device with a cm-ruler beside it. . . . . . . . . . . . . . . . . . . . 
3.4 A SEM photograph of a microchannel of the fabricated microfluidic device showing surface roughness of an etched trench (microchannel) on the top surface of the bottom slide after removing the top slide. .

3.5 SEM photographs taken of an microfluidic device that was sliced off perpendicular to a microchannel length and the top glass-slide removed. The bottom slide was vertically placed with a $45^{\circ}$ horizontaltilt. The photograph shows the surface roughness on the channel bed and the channel wall. These surfaces were imaged at a higher magnification and shown to the right. . . . . . . . . . . . . .

3.6 A photograph of the fabricated and packaged chip (lid uncovered) with a mm ruler beside it. All pins are at the bottom of the package. . . .

3.7 A SEM photograph of two electrodes of Electrode Set 3 showing little surface roughness. A scale bar is indicated. . . . . . . . . .

3.8 A photograph of a portion of the cell lysis chamber on the chip, showing a cell port, a section of a microchannel, and integrated electrodes (interdigitated and thick). The photograph was taken by a reflected light confocal optical microscope. Note that the metal layer is seen in a lighter color due to light reflection. . . . . . . . . . . . .

3.9 A sketch showing the top view of the final device. The configuration of two types of integrated electrodes are shown: i) Interdigitated electrodes (fingers), ii) Thick electrodes. Relevant dimensions of the tapered loading ports, electrodes and microchannel are indicated. .

3.10 A sketch showing the cross section of the final device along a loading port demonstrating configuration of integrated electrodes and mi-

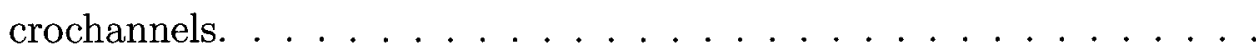

4.1 FEM simulation of an MFD without a microchannel structure. . . . 70

4.2 FEM simulation of the MFD with microchannel structures. Here electric field inside the microchannel sub-domain is only shown. . . . . 
4.3 A simple model of a rectangular box microchannel filled with D-PBS buffer fluid and electrodes on both sides. The whole structure is enclosed within a glass substrate (not shown). . . . . . . . . 72

4.4 Plot representing relationship between the developed electric field strengths $\left(E_{c h}\right)$ with the lengths of the microchannels $\left(l_{c h}\right)$ for various excitation voltages $\left(V_{a p p}\right)$. Normalized deviations $\left(\delta_{d e v}\right)$ are plotted at the bottom. All simulation results include error bars representing standard

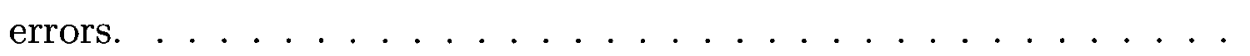

4.5 Plot depicting power dissipations $\left(P_{d}\right)$ for various area-over-length ratios $\left(A_{c h} / l_{c h}\right)$ of the microchannels for various excitation voltages $\left(V_{a p p}\right)$. Here, $\rho_{c h}=0.625 \Omega-\mathrm{m}$. Normalized deviations $\left(\delta_{\text {dev }}\right)$ are plotted at the bottom. All simulation results include error bars representing standard

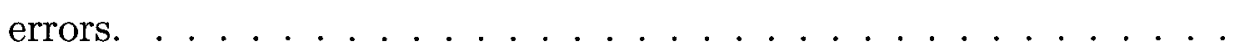

4.6 Plot showing dependance of the energy densities $\left(u_{c h}\right)$ inside the microchannels with the microchannel lengths $\left(l_{c h}\right)$ for various excitation voltages $\left(V_{a p p}\right)$. Here, $\epsilon_{r}=80$. Normalized deviations $\left(\delta_{\text {dev }}\right)$ are shown at the bottom. All simulation results include error bars representing standard errors. . . . . . . . . . . . . . . . . .

4.7 Plot representing the total energies $\left(U_{c h}\right)$ stored versus area-over-length ratios $\left(A_{c h} / l_{c h}\right)$ of the microchannels for various excitation voltages $\left(V_{a p p}\right)$. Here, $\epsilon_{r}=80$. Normalized deviations $\left(\delta_{\text {dev }}\right)$ are given at the bottom. All simulation results include error bars representing standard

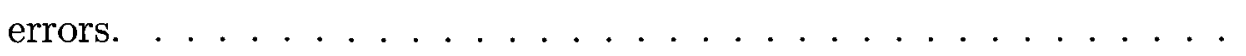

4.8 Top view of a microchannel of the MFD to be analyzed for developed

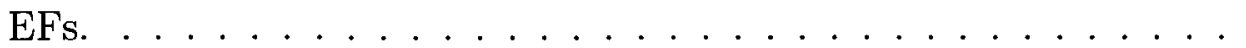
79

4.9 A 3-D view of a portion of the microchannel of the MFD shown in Figure 4.8 is depicted here. The top and bottom glass-slides that compose the glass enclosure, an electrode, an access hole, an etch-pad and a section of a microchannel are indicated. . . . . . . . . . . . 
4.10 FEM simulation of a $8 \mathrm{~mm}$ long microchannel of the MFD for an applied voltage of $800 \mathrm{~V}$. The microchannel width and height are 100 $\mu \mathrm{m}$ and $20 \mu \mathrm{m}$, respectively. (a) Color key legend of the electric field strengths. (b) and (c) Electric field distribution at the reservermicrochannel intersection (top and bottom view, respectively). (d) Electric field distribution along the microchannel and reservoirs (top view). (e) A plot of the electric field strength at the middle of the microchannel (horizontally aligned with (d)). Here electric field inside the microchannel sub-domain is only shown. . . . . . . . . .

4.11 Electric field and voltage profile inside the microchannel with electrodes positioned at various locations (location 1-1 to location 5-5) of the reservoirs are shown (vertically aligned) below the model of the MFD. Five images of various locations of electrodes are superimposed in the model. .........................

$4.12 \mathrm{~A} 3 \mathrm{D}$ view of the model of the micro-device designed using Comsol Modeler for FEM simulations. . . . . . . . . . . . . . 88

4.13 Meshed structure of the micro-device for electric field simulation. . . 88

4.14 Electric field simulation of the final device along xy-plane demonstrating development of fields of $2.5 \mathrm{MV} / \mathrm{m}$ strengths. . . . . . . . . .

4.15 Electric field simulation of the final device along xz-plane with a section (with higher mesh size) enlarged to show distribution of electric fields along the height of the microchannel. . . . . . . . . . . .

4.16 Transient development of the electric field inside the microchannel at a point $1 \mu \mathrm{m}$ above the microchannel bottom surface of the lysis chamber. The excitation was applied at time $0 \mathrm{~s}$ and continued for $10 \mathrm{~s}$. .

4.17 Meshed structure of the micro-device for thermal simulation with enlarged view of two important regions around the microchannel and the outer boundary. . . . . . . . . . . . . . . . . 
4.18 Temperature of the micro-device visualized along a xz-plane (slice plot) of the channel in the cell lysis chamber after and excitation for 10 s. A section of the channel is enlarged to depict the corresponding temperature distribution along the height of the microchannel and the

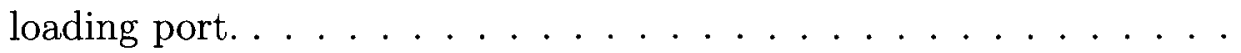

4.19 Simulation results of temperature rise inside the microchannel for lysis due to pulse excitation of $20 \mathrm{~V}$ for $10 \mathrm{~s}$. Temperature at three points were evaluated: (i) at middle of the microchannel $20 \mu \mathrm{m}$ above the bottom surface of the channel, (ii) at the top of the microchannel 40 $\mu \mathrm{m}$ above the bottom surface of the channel, and (iii) at the bottom surface of the microchannel. All three points were directly underneath the middle of a loading port where the maximum temperature variation was observed. . . . . . . . . . . . . . . . . .

4.20 A plot showing transient increase of temperature for the values of heat transfer coefficient (h) set to 5,10 and $20 \mathrm{~W} / \mathrm{m}^{2} \mathrm{~K}$, as an electric pulse excitation of $20 \mathrm{~V}$ continues for $10 \mathrm{~s}$. The initial temperature was of all sub-domain was assumed to be at room temperature of $300 \mathrm{~K}$. .

4.21 Steady state temperature simulation results showing a temperature increase up to $22.98 \mathrm{~K}$ from room temperature. . . . . . . . . 103

4.22 A section of the model is enlarged to show electrode-microchannel configuration for Joule heating coupled heat transfer multi-physics analysis. 104

4.23 Detailed view of mesh around the electrodes for the multi-physics FEM simulations. In the insets at the bottom, meshes of two sections of importance are shown. . . . . . . . . . . . . . 105 
4.24 Multi-physics simulation of Joule heating coupled with heat transfer depicting the temperature rise for a $10 \mathrm{~s}$ excitation with $20 \mathrm{~V}$ of applied voltage through the integrated electrodes. (a) Cross-section view of the microchannel at the middle of two electrodes. (b) Axial-section view of the microchannel showing temperature distribution inside the microchannel. . . . . . . . . . . . . . 106

5.1 A photograph of the custom-made platform for holding the MFD during the experiments. . . . . . . . . . . . . . 109

5.2 A schematic representation of the experimental setup of the MFD for the electrical lysis experiments. . . . . . . . . . . . . . .

5.3 Cell debris of a fingerprint sample observed under (a) $10 \times$ and (b) $40 \times$ lens. (c) Staining using Hematoxylin dye shows presence of cells (nucleic acid) within the sample. . . . . . . . . . . . 110

5.4 Average impedance microchannels of the device is plotted for a frequency range of $1 \mathrm{kHz}$ to $100 \mathrm{kHz}$ in two sets of graphs: first set showing real and imaginary components of the impedance, while the second set depicting the magnitude and angle of the impedance. In all cases, standard deviations are shown for measurement points as vertical lines. The experimental results are matched with simulation of theoretical model with a parallel combination of a resister $(R)$ of 2.7 $\mathrm{M} \Omega$ and a capacitor $(\mathrm{C})$ of $1.4 \mathrm{pF}$. . . . . . . . . . . . . 113

5.5 Plot showing average values of the decaying potentials developed in electrode-pairs for a $7 \mathrm{~mm}$ long microchannel. The error bars represent the standard deviations. The number of trials were $10 . \ldots$. . . . 
5.6 Averaged data are plotted for means (shown as bars) and standard deviations (shown as errors) for not-blocked and blocked microchannels of $7 \mathrm{~mm}$ long. The plots on the left are for measured resistances of the microchannels, and on the right are for potentials developed when electrode-pair was introduced in the reservoirs. The number of trials were $20 . \ldots \ldots \ldots \ldots \ldots \ldots$. . . . . . . . . . . . . . . . . . . . .

5.7 A fluorescence dyed cell collected from a fingerprint sample was positioned inside a microchannel and observed with a fluorescence microscope. (a) Image with normal light. (b) Image with $488 \mathrm{~nm}$ laser. Images with $10 \mathrm{X}$ magnification. . . . . . . . . . . . . . . 117

5.8 A microscopic image (10X magnification) of a section of a microchannel of the MFD showing scratches developed inside the microchannel resulted from electric arcs when $1.5 \mathrm{kV}$ was applied across the $8 \mathrm{~mm}$ long microchannel. . . . . . . . . . . . . . . . 118

5.9 Temporal sequence of microscopic images (10X lens) showing EL of a sample of fingerprint debris captured at a rate of $2 \mathrm{fps}$. Legends: asample cell exposed to a high electric field inside the microchannel, b- control cell exposed to a low electric field in the reservoir, c- the reservoir (i.e. access hole), d- the microchannel, e- the etch-pad. . . .

5.10 A snapshot captured from the oscilloscope during the application of a high voltage for EL experiments. Ch1: The applied voltage across the electrode pair $\left(V_{a p p}\right)$. Ch2: Voltage $\left(V_{R s}\right)$ developed across the series resistance, $R_{s}=10 \mathrm{k} \Omega$. $V_{R s}$ is proportional to current flow $\left(I_{c h}=V_{R s} / R_{s}\right)$ through the microchannel. Reader may refer to Figure 5.2 for the setup. . . . . . . . . . . . . . . .

5.11 An enlarged version of the temporal sequence of photographs showing the cell (a) being lysed from the time of pulse application. . . . . . 
5.12 A block diagram showing major components and interactions of the blocks of the FPGA board to generate pulse waveforms of interest for testing of the device. . . . . . . . . . . . . . . 128

5.13 The schematics of the push-pull driver stage of the drive circuitry to operate the device (by exciting the microchannel) in bipolar mode. . .

5.14 Microscopic images with 20X magnification showing bond-wire degradation due to buffer fluid in the intermediate device. (a) A good bond wire connected to a bond pad. (b) Bubbles being formed as buffer fluid overflowed and came into contact with a bond wire. (c) A partially damaged bond wire due to reaction with the buffer fluid. Note that the portion of the bond wire in contact with the buffer fluid was damaged only. (d) A completely disconnected bond wire (moved away due to strain in bond wire). . . . . . . . . . . . . . . .

5.15 Encapsulation scheme for the device showing a top view (above) and a cross-section view (below). . . . . . . . . . . . .

5.16 The heating cycle recommended for the encapsulation protocol using a metal-block in the application note from Canadian Microelectronics Corporation (CMC). In the time scale, the symbol' denotes hours and " denotes minutes of the elapsed time. . . . . . . . . . . . .

5.17 An encapsulation attempt with a metal-block according to the appnote from CMC showing different types of faults generated. . . . . . .

5.18 A photograph of the device after encapsulation with the developed protocol. The exposed area was free from polyimide leakage flow fault. The overflow and underflow faults were also minimal. . . . . . . . . 136

5.19 Section of the device after encapsulation with the developed encapsulation protocol observed under an optical microscope (10X magnification). The encapsulation boundary was sharply defined with minimal overflow or underflow fault. No leakage flow was noted. . . . . . . . 137 
5.20 A pulse of 5 second duration of $20 \mathrm{~V}$ magnitude was applied to Electrode Set $4 \mathrm{~b}$. Observability was obstructed due to formation of numerous bubbles. Microscopic images captured at 20X magnification. . . . 138

5.21 A set of microscopic images (40X magnification) showing morphological changes on fingerprint debris from an electrical pulse excitation. Here, an excitation voltage of $20 \mathrm{~V}$ was applied for 5 seconds. Note that only the debris at the high electric field region was affected. . . . 140

5.22 Microscopic images (40X magnification) showing effect of a pulse of $1 \mathrm{~ms}$ duration and $20 \mathrm{~V}$ magnitude application to the Electrode Set 4a with $100 \mu \mathrm{m}$ electrode gap. One of the debris was significantly deformed morphologically. . . . . . . . . . . . . . .

5.23 Experimental results of electrode resistance using Ohm-meter in various cell ports with respect to (wrt) electrode ports. The top portion of the figure represents relative positions of cell ports, electrode ports and fluidic ports. Maximum and minimum data are shown in a format indicated on the top right side notation box. The plots below shows averages with standard error of these data. . . . . . . . . . . . . . 144

5.24 A photograph of the developed rapid electrical lysis micro-device using SensoNit microfluidics technology. The image taken after postprocessing shows some key components of the device. . . . . . . . . 146

5.25 Images of cheek cells: (a) an unstained cell; (b) cell stained with Eosin stain only (that colors cytoplasm in pink/red color); (c) cell stained with both Eosin and Haematoxylin stains, where Haematoxylin stain colored chromatin (strands of DNA packed in nucleus) in blue color; and (d) stained cells observed after 1 week of storage. All images are taken with optical microscope in bright field mode with $40 \mathrm{X}$ objective lens. . . . . . . . . . . . . . . . . . . . . . . . 148 
5.26 Schematic diagram of experimental setup of the device. The device under test (DUT) is connected to the pulse generator and an oscilloscope through a capacitor (C) and a diode (D) . . . . . . . . . . . 148

5.27 RGB color cube with red (R), green $(\mathrm{G})$ and blue (B) axis shown. The hidden corner at $(0,0,0)$ is black color. The region of interest (Eosin stain color) is indicated. . . . . . . . . . . . . . . .

5.28 A sample showing the extraction of pixel data from an image into a plot. Here a $5 \times 5$ pixel image is illustrated to simplify the concept. Each pixel within the region of interest increments the count for that row (assuming y-axis plot is intended). Total count of the stained pixels from each row is plotted against the row (horizontally aligned, but not marked) on the right side as a line graph. . . . . . . . . . . 152

5.29 Procedure involved in extraction of pixel counts and plotting the corresponding data. The $\mathrm{VB}$ code sequentially processes all images of the captured sequence and stores data in CSV file format. A Matlab code analyzes the data of CSV files and determines the pixel counts for each row. Another Matlab code plots this extracted data and stores the total count of stained pixels. . . . . . . . . . . . . .

5.30 Extraction of pixel data from a set of images during an EP experiment on the left side and corresponding pixel data plot in overlapped form on the right side. Data was plotted along both $\mathrm{x}$ and $\mathrm{y}$-axis of the images. The images and the plots are not aligned. . . . . . . . . .

5.31 Images captured during an EP experiment are shown on the left side. Time series plot of the number of stains along $y$-axis are depicted on the right side. . . . . . . . . . . . . . . . .

5.32 Images captured during an EP experiment are shown on the left side. Time series plot of the number of stains along $\mathrm{x}$-axis are depicted on the right side. . . . . . . . . . . . . . . . . . 
5.33 Exponential decrease of Normalized Stain Reduction (NSR) of pixels over elapsed time. Data were extracted from 30 images of a sequence over 2.5 minutes duration. Total number of pixel counts were used for this plot. . . . . . . . . . . . . . . . . . 158

5.34 Two image sets (captured with 20X lens using the CCD camera attached with optical microscope) from EP experiments with $\mathrm{D}-\mathrm{H}_{2} \mathrm{O}$ as buffer medium. In the top set of pictures (Case 1: Control), stained cells retained colors (stains) after 2 minutes. In the bottom set of pictures (Case 2: Pulse excitation), Eosin stains reduced significantly when a pulse of $100 \mathrm{~ms}$ with magnitude of $20 \mathrm{~V}$ across the electrodes was applied. . . . . . . . . . . . . . . . . .

5.35 Sequence of pictures obtained from the CCD camera showing temporal changes as a $20 \mathrm{~V}$ pulse of $100 \mathrm{~ms}$ duration is applied across the electrodes at $0 \mathrm{~s}$ (Case 2 of Figure 5.34). Here stained cells were suspended on $\mathrm{D}-\mathrm{H}_{2} \mathrm{O}$ medium. . . . . . . . . . . . . . . .

5.36 Combined plot of EP and EL related result showing various excitation conditions and revenant observations. The resultant plot can be divided into 3 regions: No effect observed, EP region and EL region. .

5.37 Normalized stain reduction with time for various excitation condition with $5 \mathrm{~V}$ of applied potential. The NSR plots are averaged over 3 samples. The control case is not excited by any electrical pulse.

5.38 Normalized stain reduction with time for various excitation condition with $20 \mathrm{~V}$ of applied potential. The NSR plots are averaged over 3 samples. The control case is not excited by any electrical pulse. . . . 167

5.39 Normalized stain reduction with time for various excitation voltage with the same pulse parameters: 1 pulse of $10 \mathrm{~ms}$ duration. The NSR plots are averaged over 5 samples. The control case is not excited by any electrical pulse. . . . . . . . . . . . . . . 
5.40 Microscopic images (10X lens) showing cell structures were broken and cytoplasm released to buffer medium as a pulse of $20 \mathrm{~V}$ magnitude and $500 \mathrm{~ms}$ duration was applied. The release of cytoplasm concluded from the released Eosin stains that are observed to be diffused in (b). . . .

5.41 Observation under a microscope (10X magnification) after applying a single pulse of $20 \mathrm{~V}$ magnitude and $1 \mathrm{~s}$ duration. (a) Image prior to excitation (at $0 \mathrm{~s}$ ). (b) Image immediately after excitation (at 1 s). Bubbles due to excitation limited visible area. (c) Superimposed images obtained by subtracting image (b) from image (a) and enlarged to show the loading port. . . . . . . . . . . . . . 172

5.42 Two microscopic images (40X magnification) taken from the same spot, but focused at two different heights (about $10 \mu \mathrm{m}$ apart), which show exposed DNA through EL with a $20 \mathrm{~V}, 5 \mathrm{~s}$ excitation. The cells were stained with Hematoxylin (stains DNA in blue-purple color) and Eosin (stains cytoplasm in pinkish-red). The images show exposed DNA and de-stained cell membrane. Here the image (b) is focused about $10 \mu \mathrm{m}$ above (a). The dotted areas are zoomed in the next figure to highlight the differences of captured DNA images. . . . . . . . . . .

5.43 Enlarged images showing exposed DNA at two vertical heights within the micro-device. The difference of height between the images is about $10 \mu \mathrm{m}$. The arrows indicate intensity differences between the images taken from the same spot, demonstrating that DNA stands were distributed vertically in spatial domain compared to electrodes. . . . . .

5.44 One of the images of exposed DNA is processed to reduce background color using standard image processing technique, and shows stained DNA clearly. . . . . . . . . . . . . . . . . .

5.45 Rearranged cell membrane observed under the optical microscope (20X lens) that is apparently in complete irregular shape observed after EL experiments. . . . . . . . . . . . . . . . 177 
5.46 Two samples of exposed DNA observed with 100X objective lens (oil immersion) in collected fluids from the micro-device after excitation of $20 \mathrm{~V}$ for $5 \mathrm{~s}$. The DNA strands are identified by the blue stain of Haematoxylin and the coiled structure that is unique to DNA stands (chromatin) . . . . . . . . . . . . . . .

5.47 Microscopic images (20X lens) showing two types of defects developed through electrode degradation inside the device: Crack and Lift off. .

5.48 Sketches showing effect of applying above critical electric field pulses of different widths on a human cell causing electroporation, electrical lysis and nucleus membrane disintegration. . . . . . . . . . . .

6.1 A state diagram representing the sequence of events involved in the dynamics of EL process. Here, the states are represented as rectangles and transitions are indicated using arrows. The solid arrows represent transitions due to application of an electric field above the threshold value. The hollow arrows represent spontaneous transitions after the applied electric field is withdrawn. The process is reversible until the critical pore diameter, after which pores grow spontaneously and an irreversible process is initiated. The zone between pore formation and pores attaining critical diameters is denoted by EP region, and the zone beyond the critical pore diameter is termed as the EL region .

6.2 Entropy of a cell membrane structure is drawn qualitatively as a function of elapsed time $(t)$. The paths denote traces of entropy for various applied electric field or traces as the electric field is withdrawn. The nucleation periods are denoted $\left(t_{n 1}, t_{n 2}, t_{n 3}\right)$. The critical times (eg. $t_{c 1}$, $\left.t_{c 2}, t_{c 3}\right)$ to achieve critical pore diameter reduce with increasing electric field strengths (i.e. $E_{t h 1}, E_{t h 2}, E_{t h 3}$ ) above the threshold electric field $\left(E_{t h}\right) .($ Adapted from [1].) . . . . . . . . . . . . 184 
6.3 Reorientation of phospholipids of a bilayer membrane in hyperpolarized and depolarized side of a cell under a threshold electric field $(\boldsymbol{E})$ causing pores to form. The arrows in (b) and (c) indicate the directions of the applied electric field. (Adapted from [2,3].) . . . . . . . . 186

6.4 Current electric circuit model of a cell, where the cell membrane is represented by a parallel combination of a capacitor $\left(C_{m}\right)$ and a resistor $\left(R_{m}\right)$, while the cytoplasm inside the cell is modelled with a resistor

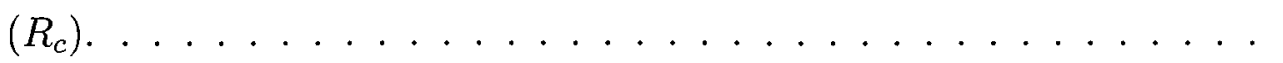

6.5 Cylindrical model of a cell converted from the spherical cell that allows determination of model parameters of the electrical circuit model. . . 188

6.6 The complete electrical circuit including a model of a cell inside a micro-channel ........................... 191

6.7 A cylindrical model of cellular membrane pores formed during EP. . . 193

6.8 Comparison of total average membrane resistance $\left(R_{T M}\right)$ calculated using the cylindrical pore model and the electrical circuit model for typical pore diameters and the number of pores $\left(\mathbf{n}_{p}\right) \ldots \ldots$. . . .

6.9 The electrical model of transmembrane resistance due to reversible pores formed during EP. The membrane resistance between N1 and $\mathrm{N} 2$ reduces drastically as the potential reaches the threshold transmembrane potential. ...................

6.10 The complete circuit diagram for simulation of a cell inside a microchannel using (a) the current pore model with a fixed resistance model (2RM), and (b) the proposed pore model (X1). Here the cell is modelled using $\mathrm{RC}, \mathrm{CM} / 2$, the microchannel is modelled using $\mathrm{RSB}$, $\mathrm{RPB}$, and CB, and the power supply is represented by V1, and RSS.

6.11 Simulation result of a cell exposed to 2 pulses of $1 \mu$ s duration using the current pore model. Here, V(1) represents the applied voltage, V(4)/2 represent transmembrane potential, and $\mathrm{V}(4) /(2 * \mathrm{I}(2 \mathrm{rm})$ represents the membrane resistance. . . . . . . . . . . . . . . . . 
6.12 Simulation result of a cell exposed to 2 pulses of $1 \mu$ s duration using the developed pore model. Here, V(1) represents the applied voltage, $\mathrm{V}(4) / 2$ represent the transmembrane potential, and $\mathrm{V}(4) /\left(2^{*} \mathrm{I}(\mathrm{X} 1: \mathrm{N} 1)\right.$ represents the membrane (pore) resistance. . . . . . . . . . . 200

6.13 Simulation results of a cell exposed to a single pulse of $2 \mu \mathrm{s} \mathrm{du}-$ ration using the developed pore model. Here, V(1) represents the applied voltage, $\mathrm{V}(4) / 2$ represent the transmembrane potential, and $\mathrm{V}(4) /\left(2^{*} \mathrm{I}(\mathrm{X} 1: \mathrm{N} 1)\right.$ represents the membrane (pore) resistance. . . . . 201

6.14 Simulation result of a cell exposed to 2 pulses of $1 \mu$ s duration using the developed pore model where no EP occurred, as the applied voltage is low. Here, $\mathrm{V}(1)$ represents the applied voltage, $\mathrm{V}(4) / 2$ represent the transmembrane potential, and $\mathrm{V}(4) /\left(2^{*} \mathrm{I}(\mathrm{X} 1: \mathrm{N} 1)\right.$ represents the membrane (pore) resistance. . . . . . . . . . . . . . 202

6.15 Plot of Degree of EP from various simulation cases are shown. The excitations for various simulation cases were: (1) $10 \mathrm{~V} 1 \mu \mathrm{s} 2$ pulses, (2) $10 \mathrm{~V} 2 \mu \mathrm{s} 1$ pulse, (3) $20 \mathrm{~V} 1 \mu \mathrm{s} 2$ pulses, (4) $20 \mathrm{~V} 2 \mu \mathrm{s} 1$ pulse, (5) $20 \mathrm{~V} 1 \mu \mathrm{s} 5$ pulses, and (6) $20 \mathrm{~V} 5 \mu \mathrm{s} 1$ pulse. . . . . . . . . 206

6.16 Plot of EFEP from various simulation cases are shown. The excitation cases were: (1) $10 \mathrm{~V} 1 \mu \mathrm{s} 2$ pulses, (2) $10 \mathrm{~V} 2 \mu \mathrm{s} 1$ pulse, (3) $20 \mathrm{~V} 1$ $\mu \mathrm{s} 2$ pulses, (4) $20 \mathrm{~V} 2 \mu \mathrm{s} 1$ pulse, (5) $20 \mathrm{~V} 1 \mu \mathrm{s} 5$ pulses, and (6) 20 $\mathrm{V} 5 \mu \mathrm{s} 1$ pulse.

6.17 Plot of Degree of EP for a range of applied voltage is plotted from simulation results. Three different cell size results are shown. . . . . .

6.18 Plot of Degree of EP versus different cell diameters for various applied voltages. ....................... 208

6.19 Plot of EFEP for a range of applied voltage plotted for different cell diameters. Pulse widths were $1 \mu \mathrm{s}$. . . . . . . . . . . 208

6.20 Plot of EFEP for different cell diameters with various excitation volt-

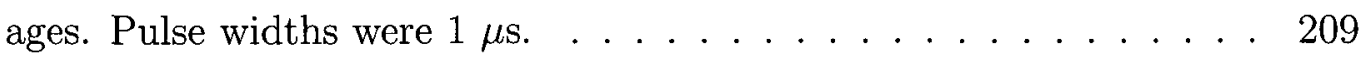


6.21 Plot of Degree of EP for various number of pulses with different total pulse durations. For multiple pulses, pulse gap was equal to pulse width.210

6.22 Plot of EFEP for various number of pulses delivering the same total energy with different total pulse durations. For multiple pulses, pulse gap was equal to pulse width. . . . . . . . . . . . 210

A.1 Full layout of the initial device. . . . . . . . . . . . . . . 222

A.2 Left hand side section of the initial device is enlarged to show details of the layouts of the microchannels and reservoirs. . . . . . . . . . .

A.3 Right hand side section of the initial device is enlarged to show details of the layouts of the microchannels and reservoirs. . . . . . . . . . .

A.4 Layout of the complete MetalMUMPs chip showing different important elements of the design. Mask layer legends is shown at the bottom of the layout. . . . . . . . . . . . . . . . . . . 225

A.5 Layout of a section of Electrode Set 1 in the chip. . . . . . . . . 226

A.6 Layout of Electrode Set 2 in the chip. . . . . . . . . . . . 226

A.7 Layout of a section of Electrode Set 3 in the chip. . . . . . . . . . 227

A.8 Layout of a section of Electrode Set 4 a in the chip. . . . . . . . . 227

A.9 Layout of the Sensonit device showing all 3 masks, electrode mask, microchannel mask, and port mask. The large green area on the lower half of the chip constitutes the cell lysis chamber with interdigitated electrodes across microchannels with cell ports above them. The ruler shown is in $\mu \mathrm{m}$. The cell lysis chamber is indicated and a section is marked as A, which is enlarged in Figure A.10. . . . . . . . .

A.10 An enlarged version of the layout of section $\mathbf{A}$, showing portion of the cell lysis chamber (the section marked as A in Figure A.9). A cell port, some interdigitated electrodes (fingers) on a microchannel and two thick electrodes are indicated. A scale bar is shown at the bottom. 230 
B.1 A photograph showing the components and tools needed for the developed fluidic encapsulation protocol. . . . . . . . . . . . . . . 232

B.2 A photograph showing a test to verify proper connectivity of an electrode with the integrated electrodes after silver epoxy bonding. . . . . 236

B.3 A photograph showing procedure for reconnecting the electrode on a sample device. The post-processed device has fluidic nano-port bases fixed. The masking tape covers all cell loading ports to prevent contamination and will be removed after post-processing is done. . . . . 238

C.1 The pulse amplitude is being set in the FPGA board. . . . . . . . . 251

C.2 The number of recitation of pulses is being set in the FPGA board. . 251

C.3 Time duration for OFF period in between pulses (i.e. pulse gap) is being set in the FPGA board. . . . . . . . . . . . . . 251

C.4 Time duration for ON period (i.e. pulse width) is being set in the FPGA board. . . . . . . . . . . . . . . . . . 251

C.5 The FPGA board in RUN state delivering pulses through the GPIO ports. . . . . . . . . . . . . . . . . . 252

C.6 A snapshot of the visual basic (VB) program interface. . . . . . . 259 


\section{List of Tables}

1.1 A comparison of several fabrication technologies on the basis of our research interest to develop a rapid and portable cell lysis device. .

2.1 A chronological list of major findings related to electroporation (EP) and electrical lysis (EL) . . . . . . . . . . . . . . . . . 34

3.1 A table showing various mask layers of the MetalMUMPs fabrication process and brief descriptions of layers. [Adapted from MetalMUMPs Design Handbook $]$. . . . . . . . . . . . . . . . . .

4.1 Maximum values of the normalized standard errors from FEM simulations of the simple model and the MFD model . . . . . . . . . . . .

4.2 The range of variables for the estimation error ranges when calculating various parameters (electric field, power dissipation, energy density and total energy stored) for the microchannel-based MFDs using the derived analytical expressions $\ldots \ldots \ldots \ldots \ldots$

5.1 Observations under various electric field strengths . . . . . . . . . 119

5.2 Average values of various parameters from the electrical lysis experi-

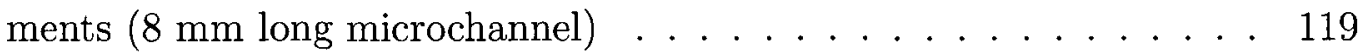

5.3 Summary of experimental parameters and corresponding observation using the final device . . . . . . . . . . . . . . . . . . . . . . 163 
6.1 Model parameters used to calculate component parameters of the electric circuit model from literature $[4] \ldots \ldots$. . . . . . . . 192

6.2 Degree of EP and EFEP from experimental results (1 pulse of $10 \mathrm{~ms}$ duration) . . . . . . . . . . . . . . 213

6.3 Degree of EP and EFEP from experimental results (5 V applied potential) 214

6.4 Degree of EP and EFEP from experimental results (20 V applied potential) . . . . . . . . . . . . . . . . . 214

7.1 Feature comparison of the final device of this work with others from the literature . . . . . . . . . . . . . . . . . 220 


\section{List of Abbreviations and}

\section{Acronyms}

CE

Capillary Electrophoresis 
DDBJ

D- $\mathrm{H}_{2} \mathrm{O}$

DMPC

DNA

D-PBS

DPDT

DUT

EFEP

EL

EP

FBI

FEM

FPGA

GEN2PHEN

GPIO

H\&E

LOC

LTCC

MD

MEMS
DNA Data Bank of Japan .2

Deionized Water. 234

1,2-dimyristoyl-sn-glycero-3-phosphorylcholine

Deoxyribonucleic Acid . 122

Dulbecco's Phosphate Buffered Saline 108

Double pole double throw. 127

Device Under Test 129

Electroporation Fluid Exchange Parameter. . 219

Electrical Lysis 182

Electroporation 182

Federal Bureau of Investigation ....................... 2

Finite Element Method 217

Field-Programmable Gate Array . . . 126

Genotype-to-Phenotype .2

General purpose input output. 127

Hematoxylin and Eosin 146

Lab-on-a-chip.

Low Temperature Co-fired Ceramic 12

Molecular Dynamics 43

Microelectromechanical System . . . 56

MetalMUMPs Metal Multi-User MEMS Processes 222

MFD Microfluidic Device

$\mu$ TAS

Micro Total Analysis System 45

MUMPs

Multi-User MEMS Processes 14 
NSR Normalized Stain Reduction ......................... 212

NTP Normal Temperature and Pressure $\quad \ldots \ldots \ldots \ldots \ldots \ldots \ldots . \ldots 43$

PCR Polymerase Chain Reaction ........................ 107

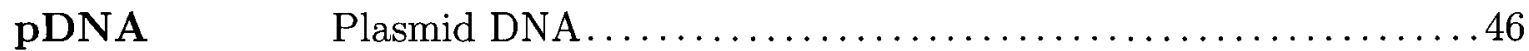

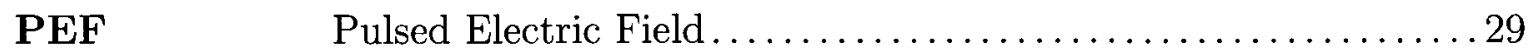

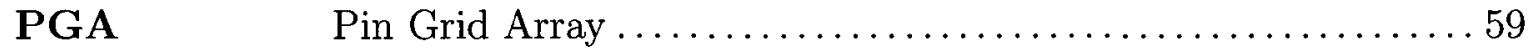

RAM Random access memory...........................

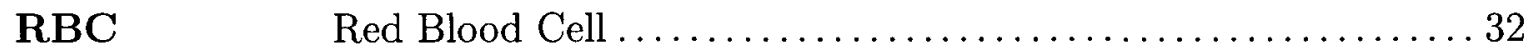

RTL Register transfer level............................. 129

SEM Scanning Electron Microscope ...................... 109

siRNA Small Interfering Ribonucleic Acid .................... 46

SNP Single Nucleotide Polymorphism $\ldots \ldots \ldots \ldots \ldots \ldots \ldots \ldots \ldots$

STR $\quad$ Short Tandem Repeat..............................

VB Visual Basic .................................. 149

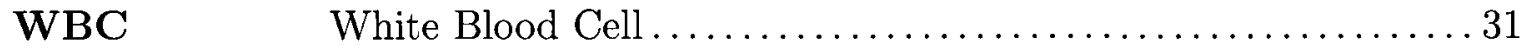


$\mathrm{XxXV}$ 


\section{Notations and Symbols}

a Cell radius

$A_{c h} \quad$ Cross-sectional area of a microchannel

$a_{i} \quad$ Radius of the organelle

$\alpha \quad$ Ratio of channel resistance to total reservoir resistances

c Proportionality constant

C Capacitor

$C_{B} \quad$ Capacitance of buffer solution

$C_{c h} \quad$ Capacitance of microchannel

$C_{m} \quad$ Membrane capacitance

$d \quad$ Diameter of a cylindrical cell

$d_{m} \quad$ Thickness of cell membrane

$\delta \quad$ Angle between $E$ and the radius vector to $V_{m}$

$\delta_{E P} \quad$ Degree of electroporation

$\delta_{\text {dev }} \quad$ Normalized deviations (estimation errors)

$E \quad$ Electric field strength

$E^{\prime} \quad$ Electric field strength related constant

$E_{c h} \quad$ Electric field inside a microchannel

$E_{i} \quad$ Electric field calculated using simplistic expression

$E_{i}(t)$ Time-varying intracellular electric field

$E_{m} \quad$ Transmembrane electric field strength

$E_{m} c \quad$ Maximum transmembrane electric field strength

$E(r)$ Pore energy function 
$E(r, t)$ Time-varying pore energy function

$E_{s} \quad$ Step function electric field strength

$E_{t h} \quad$ Threshold electric field strength

$\epsilon \quad$ Permittivity of the buffer fluid

$\epsilon_{0} \quad$ Permittivity of the free space

$\epsilon_{B} \quad$ Relative permittivity of buffer

$\epsilon_{m} \quad$ Relative permittivity of cell membrane

$\epsilon_{r} \quad$ Relative permittivity of the buffer fluid

$f \quad$ Frequency of operation

$f_{f} \quad$ Form factor

$f_{p} \quad$ Pulse rate

$\gamma \quad$ Energy per unit length of the pore perimeter

$G_{m} \quad$ Membrane conductance per unit area

$G_{s} \quad$ Surface conductance of the membrane

$h \quad$ Thickness of the cell membrane

$h_{c} \quad$ Height of the cell in the cylindrical model

$h_{c h} \quad$ Height of a microchannel

$i \quad$ Amount of current flow

$I_{0} \quad$ Modified Bessel function of the zeroth order

$I_{1} \quad$ Modified Bessel function of the first order

$\zeta \quad$ Entropy of membrane structure

$\zeta_{E P} \quad$ Electroporation Fluid Exchange Parameter

$K \quad$ A proportionality constant

$l \quad$ Length of a cylindrical cell

$l_{c h} \quad$ Length of a microchannel

$m \quad$ Slope

$n_{p} \quad$ Number of pulses

$\mathbf{n}_{p} \quad$ Number of pores 
$n(r, t) \quad$ Pore distribution function

$\omega \quad$ Angular frequency of the applied potential

$P_{d} \quad$ Ohmic power dissipation

$\phi \quad$ Transmembrane potential

$r \quad$ Average pore radius

$r_{a} \quad$ Radius of cell in spherical model

$r_{c} \quad$ Radius of cell base in cylindrical model

$R \quad$ Resistor

$r_{0} \quad$ Characteristic length scale

$R_{c} \quad$ Resistance of cytoplasm

$R_{c h} \quad$ Resistance of a microchannel

$r_{p} \quad$ Pore radius

$R_{p} \quad$ Resistance of membrane pore

$R_{m} \quad$ Resistance of cell membrane

$R_{P B} \quad$ Buffer resistance in parallel to cell

$R_{\text {res }} \quad$ Resistance of a reservoir

$R_{s} \quad$ Series resistance

$R_{S B} \quad$ Buffer resistance between cell and electrode

$R_{S S} \quad$ Resistance of supply

$r(t) \quad$ Time-varying average pore radius

$R_{T M} \quad$ Total transmembrane resistance

$\rho_{B} \quad$ Resistivity of buffer

$\rho_{c} \quad$ Resistivity of cytoplasm

$\rho_{i} \quad$ Resistivity of intracellular medium

$\rho_{m} \quad$ Resistivity of cell membrane

$\rho_{o} \quad$ Resistivity of extracellular medium

$\rho_{c h} \quad$ Resistivity of the buffer fluid

$s \quad$ Survivability

$\sigma \quad$ Energy per unit area of the intact membrane 
$\sigma_{c h} \quad$ Conductivity of the buffer fluid

$T \quad$ Characteristic time period

$t_{c} \quad$ Critical time duration

$t_{p} \quad$ Pulse duration (or width)

$\tau^{\prime} \quad$ Charging time related constant

$\tau_{m} \quad$ Charging time constant of plasma membrane

$\tau_{m}^{\prime} \quad$ Discharging time constant

$\tau_{i m}$ Charging time constant of the organelle membrane

$\theta \quad$ Angle between electric field line and radius vector

$\theta_{i m} \quad$ Position angle on the organelle membrane

$V_{0} \quad$ Transmembrane potential under constant electric field

$V 1 \quad$ Applied voltage

$V_{a p p} \quad$ Applied voltage

$V_{c} \quad$ Critical transmembrane potential

$\mathbf{V}_{c} \quad$ Volume of cell

$V_{c h} \quad$ Voltage across a microchannel

$\mathbf{V}_{c h} \quad$ Volume of a microchannel

$V_{f} \quad$ Transmembrane potential under alternating electric field

$V_{i m} \quad$ Internal transmembrane potential

$V_{m} \quad$ Transmembrane potential

$V_{m}(t)$ Time-varying transmembrane potential

$V_{p p} \quad$ Pick-to-pick supply voltage

$V_{R s} \quad$ Voltage developed across a resistor $R_{s}$

$V_{s} \quad$ Power supply voltage

$u_{c h} \quad$ Energy density inside a microchannel

$U_{c h} \quad$ Total energy stored

$W \quad$ Dissipated electrical energy

$w_{c h} \quad$ Width of a microchannel 


\section{Chapter 1}

\section{Introduction}

This collaborated research was conducted as a part of a larger research project undergoing at Medical Devices Center of Ottawa Heart Institute. This introductory chapter provides relevant background, objective, rationale, research approach, key findings, achievements and contributions of this research.

\subsection{Overall Project of a DNA Detection Device}

Biometric identification and bio-security are of rising research interest [1-5]. Traditionally, biometric identification of a person requires observation of a physiological feature (such as face, fingerprint impressions, or retina scanning) or detection of a behavioral feature (such as signature, or voice recognition) [5]. All of these biometric identification, however, are prone to some level of detection error due to statistical matching probability. The ultimate biometric identification with total confidence can be achieved by Deoxyribonucleic Acid (DNA) profiling [6-8]. Any individual can be uniquely identified by detecting some specific DNA sequence known as Short Tandem Repeat (STR) within human genome through DNA profiling [6,9]. This detection can be performed with cells from any part of the body, because DNA in all cells (except sexual reproduction cells) throughout the human body of an individual is 
identical, but variable between any two humans - a unique characteristic [10-12]. Thus DNA based identification can play pivotal role in bio-security applications, criminal investigations and wrongful convictions [13]. Several countries, including USA, Canada, Australia, are building DNA databases for criminal identification and other purposes, such as Combined DNA Index System (CODIS) project of Federal Bureau of Investigation (FBI) or DNA Data Bank of Japan (DDBJ) holding millions of records $[7,14,15]$. In addition, DNA profiling enables various genetic analysis by Single Nucleotide Polymorphism (SNP) $[16,17]$. Besides, many characteristics and diseases are potentially related to specific human genes [18]. Example of such application is an 11.889 million Euro-funded Genotype-to-Phenotype (GEN2PHEN) project by European Commission [19].

\subsubsection{Conventional DNA detection process}

Detection of DNA is a complex procedure of sequential steps as outlined in Figure $1.1[10]$. These steps are:

1. The first step is to collect sample cells from the subject (whose DNA is to be detected). For humans, usually invasively collected blood samples are used due to technological challenges involved with DNA detection from non-invasively collected samples. The collected sample is usually sent to a lab for further processing.

2. To access internal contents of cells such as DNA, cell membranes have to be ruptured. This process is called cell lysis (or cell disruption). This step releases DNA and is commonly performed in labs with chemical lysing agents, such as Chelex, and QUIAmp [20].

3. The next step is to extract the released DNA after cell lysis. This step uses Proteinase $\mathrm{K}$ to denature binding histone proteins from DNA. 


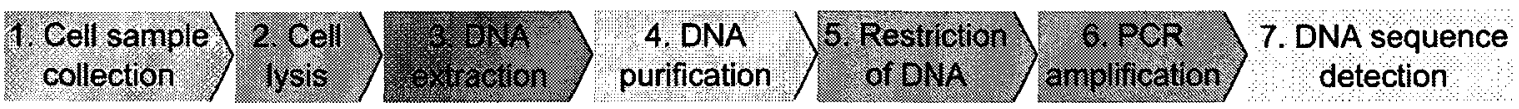

Figure 1.1 Steps involved in current DNA detection procedure.

4. To purify DNA from released proteins, cell membranes and other organelles in cell lysate, DNA binding chemical (eg. isopropyl alcohol) is added to the solution and centrifuged at a high speed to pallets pure DNA, which is collected after discarding the supernatant.

5. Next step is to restrict long strands of DNA in chromosomes to small fragments to find specific segments or genes of interest. Restriction is performed by adding restriction enzymes.

6. The resulted fragments of DNA are copied using Polymerase Chain Reaction (PCR) method. PCR is based on repeated heating cycles that uses primers, nucleotides, and enzymes and results billions of copies of the DNA fragment [21].

7. The final step is to detect strings of amplified DNA. DNA detection is usually performed with Gel Electrophoresis [22]. Recently advanced micro-fabricated devices have been developed for rapid DNA sequencing, such as DNA sequencer bio-disk [23] or DNA array chip [24,25].

As discussed, current technology for DNA detection require distinct, complicated, multi-tube procedures, expensive equipment, significant amount of time, human expertise, and invasively collected cell samples $[21,26]$. Due to the requirement of human expertise and multi-tube transfer process involving large, expensive equipment, no point-of-care device for DNA detection is available currently $[6,27,28]$. Furthermore, existing technology requires invasively collected blood sample cells as the source of DNA, which risks contamination and medical hazard. An estimated $10 \%$ adult population have Trypanophobia, a trauma effect that occur to individuals while collecting blood samples [29]. 


\subsubsection{Advancements in DNA detection process}

DNA detection process is one of the fields that received lots of recent attention from researchers. Various steps of this process have been successfully implemented onchip. One of such advancement is the development of DNA sensor array (or DNA microarray or DNA-chip) technology $[8,24,25,30-33]$. Some other areas of the DNA detection process reported recently using on-chip implementation are BioFET [34], Capillary Electrophoresis (CE) [35], genetic analysis [36-38], micro-heater [39], PCR for DNA amplification [40-47], PCR-CE integration [48], DNA separation and purification by CE $[49,50]$, DNA sequencer or bioprocessor $[23,51,52]$, and nanopore-based transducers for fast DNA detection [33,53-55].

\subsubsection{Limitations of current DNA detection process}

The existing DNA detection process suffers from the following shortcomings.

a) Invasive sample requirement: Most commercially-available equipment use blood as sample source for human DNA analysis $[6,56]$. This invasive sample requires expertise (health-care professional) to collect samples, in addition to risks of contaminations and hazards.

b) Non-portable devices: Currently used commercial DNA detection devices are non-portable [28]. Though much research is ongoing, a rapid and portable solution has still not been developed. A recent successful demonstration of a rapid and portable device developed by FBI required 2.5 hours to complete the process of DNA analysis and is carried on a vehicle [6]. Important issues to resolve for a point-of-care solution are as follows:

1. Power consumption is an important issue for portable devices. Existing solutions require large amount of power that is not available using any portable power supply. 
2. Speed of operation is another issue with such a solution. Current methods are slow (several hours to even days) that is unsuitable for a portable device.

3. Portability and weight of all components required in every step of the process must comply within some constraints. It is of utmost importance for such elements to be compatible with each other for the complete solution to be implementable on a microfluidic platform [28].

c) No single device solution: There is a need to include all relevant elements of DNA detection process within a complete packaged solution that does not exist at present. Various elements (or components) for different steps have been reported recently with on-chip solution (as mentioned in the last subsection); however, a complete packaged solution of the whole process is not reported yet.

\subsubsection{DNA detection device project}

The overall project undergoing at Medical Devices Center of Ottawa Heart Institute aims to develop a rapid and portable DNA detection device that uses non-invasive samples, such as fingerprint debris. Application area for such a device can be identification and biosecurity purpose, genetic research, criminal investigation and forensic analysis. Desired characteristics of the device sought includes:

1. Rapid operation.

2. Able to detect DNA from non-invasive samples.

3. Hand-held portability with little power consumption, suitable for point-of-care devices.

4. Complete system integration with one package solution.

5. Inexpensive, reliable and reusable. 
A proposal of the overall project to develop a rapid and portable DNA detection device from non-invasive cell samples, like debris of human fingerprints, was formulated by Professor Mussivand about a decade ago. One of the critical steps identified in the DNA detection process is release of DNA through lysis (rupture) of cell membranes. This step, which is the first major step of the process, is especially crucial for accessing DNA from minute amounts of cells collected from non-invasive samples, such as human fingerprints [29,57]. Conventional methods required expensive equipment, large amount of sample cells and multiple tube transfer $[26,57,58]$. Hence, developing a method for rapid lysis of non-invasive samples has been prioritized in this project. A method using ultrasound for cell lysis has been developed and patented that is able to obtain DNA from fingerprints within 10 minutes [59]. An even faster and portable method is desired, which led to this study of cell lysis with high electric fields.

\subsubsection{Rationale of this research}

As the existing cell lysis for DNA extraction methods are slow and complicated (discussed in the next section), Dr. Mussivand's research group focused to develop methods for faster and effective cell lysis and DNA extraction. Previously a method was developed by this research team to extract DNA from fingerprint samples within 10 to $15 \mathrm{~min}[29,57,59]$. However, another method was still sought that resolves the following persistent issues leading to this research work:

a) Faster operation: A faster solution was needed that can perform the step within seconds.

b) Non-invasive sample: The method must be able to handle minute amount of non-invasive, as well as invasive, sample cells.

c) Portability: For implementation on a portable device, the operation should consume little power and the device should be small in size and weight. Thus, a micro-device was needed to be developed that would consume little amount of 
power for cell lysis.

d) On-chip solution: To be able to package the complete DNA detection process, each element was required to be compatible with portable solution, preferably on-chip operation. Hence, an on-chip solution for cell lysis was desired.

\subsection{Overview of This Research Work}

In this section, the research objective, rationale, and challenges are discussed.

\subsubsection{Objective and scope}

The objective of this study was to develop a device and a method for cell lysis to extract DNA that can be integrated with the rapid and portable DNA detection device. Following three core requirements were targeted to be fulfilled:

1. Cell lysis to be performed rapidly (less than a minute).

2. The device must be suitable for a portable device.

3. The method must be able to use non-invasively collected cell samples.

The scope of this research was limited to only cell lysis step of the DNA detection process. The step begins after non-invasively collected cells are sampled and terminates after lysis of cell samples that releases DNA.

\subsubsection{Some relevant terms and definitions}

Some relevant terms and definitions pertinent to this research are defined briefly here for the sake of clarity.

Cell lysis: Cell lysis is defined as the destruction of cells through rupturing its plasma membrane, often by external physical or chemical stimulus, and consequently releasing the contents of the cell [11]. 
Electroporation: Formation of a large number of reversible pores in the plasma membrane and resealing of pores due to a Pulsed Electric Field (PEF) is called electroporation (EP) [1]. This is also known as electropermeabilization [60, 61], dielectric breakdown [62], or reversible electric punch-through [63]. Cell membranes made permeable through EP are called electroporated cells.

Electrical lysis: If pores formed during EP are allowed to grow beyond the critical pore diameter by continued excitation, these pores spontaneously expand to disintegrate membrane structure. Such disintegration of the plasma membrane using a high electric field is termed as Electrical Lysis (EL) [64-66]. This is also sometimes referred to as electrical breakdown $[67,68]$.

Microfluidics, MEMS and BioMEMS: Microfluidics is defined as the process that deals with a fluid volume of microliter or less [27]. Microfluidic devices are commonly fabricated using Microelectromechanical System (MEMS) technology [69]. MEMS devices are fabricated using micro-machining (bulk and surface) in conjugation to electronic device fabrication process and usually involve sacrificial layers to realize mechanical structures. MEMS devices combine more than one elements from electrical, mechanical, thermal, magnetic, fluidic and optical components on a single chip [70,71]. Biomedical Microelectromechanical System (BioMEMS) is a subset of MEMS devices specifically designed for applications in biomedical research and medical micro-devices [27,28]. BioMEMS devices enabled micro-scale microfluidic applications such as Micro Total Analysis System ( $\mu$ TAS) and Lab-on-a-chip (LOC) [28,72-74].

Buffer solution: The primary purpose of a fluidic buffer solution is to control $\mathrm{pH}$ of the solution [75]. Buffers also play role as a fluidic medium in suspending and transporting biological elements within a microfluidic device. 


\subsubsection{Current cell lysis methods}

To resolve the objectives of this research, all existing cell lysis methods were studied and compared. All of these methods for cell lysis use either physical or chemical stimulus. Methods employing physical stimulus include mechanical shear, liquid shear, gaseous shear, osmotic pressure, and sonication [58]. Methods based on chemical stimulus for cell lysis are non-ionic detergents and lysis-agents, such as Chelex, and QIAamp [20]. These chemical lysis-agents are the most commonly-used laboratory benchmark for DNA analysis at present $[20,76]$. However, chemical lysis is a slow process (tens of minutes to hours), and requires multiple tube transfer $[26,57,58]$. In addition, this method might produce contaminated and degraded lysate if the stimulus remains active even after lysis is complete $[20,58,77]$. Hence chemical lysis method did not meet the target requirement for the proposed rapid cell lysis device. In fact, none of the current methods could satisfy all three requirements to meet the objective of this research.

\subsubsection{Proposed cell lysis method}

There are a number of emerging cell lysis methods being researched that can be potentially applied to develop the proposed on-chip cell lysis device [78]. Some of these emerging cell lysis methods are laser-based cell lysis device $[79,80]$, microbead lysis [81], electrical cell lysis device [63-66,82-88], electro-osmotic cell lysis [89], thermal lysis [90], electro-chemical stimulus [91-93], or a combination of electrical and fluidic pressure stimulus [94].

Laser method requires less time compared to most other methods, but demands expensive laser source and manual intervention for focusing the beam [80], which is not suitable for a portable, automated device. To be able to lyse many cells, cells has either to be flown through a narrow channel with laser excitation or laser has to be scanned for the lysis chamber, both would require much longer time than single cell lysis time. 
Electrical lysis (EL) technique is the fastest and an inexpensive solution, and suitable for on-chip implementation. In fact, laser method finds more application for in vivo treatment of cells [95], whereas electric method is more effective for in vitro cell lysis [63]. Hence, we pursued EL method for rapid lysis on a portable device using non-invasive sample cells.

To understand and optimize electric lysis parameters, studying of electroporation (EP) is important as EP is the transient phenomenon that leads to EL. Knowledge acquired through EP experiments are transferable to EL due to the same dynamics involved in both method. In addition, studying of EP is also important due to other applications like drug delivery and gene transfection. Thus in this thesis work, much attention was given to study EP in addition to EL.

\subsubsection{Challenges and technology gap}

Several challenges and technology gap existed to develop the targeted device and method:

1. Even if breakdown of cell membrane using a high electric field was demonstrated, breakdown of nuclear membrane was never demonstrated. However to access DNA from human cells, both the cell membrane and the nuclear membrane must be disintegrated. It was hypothesized that nuclear membrane should show similar phenomenon as cell membrane with a higher electric field or a longer excitation time, as nuclear membrane is a double bilayer structure of lipids, whereas cell membrane is a single bilayer structure.

2. Previously demonstrated EL devices primarily used microorganisms, like $E$. coli or protoplast, which do not have cytoskeleton [58]. Human cell membranes, especially non-invasively collected cell samples contain cytoskeleton that strengthen the cell membrane. Thus it is expected that these cells to be difficult to lyse and will require a higher electric field or longer pulse. 
3. EL require a high electric field to be developed around the sample cell. However for a portable device, high voltage power supply is challenging to implement. To be able to use low voltage power supply, the exciting electrodes must be fabricated at micron gaps.

4. All previous EL devices required time consuming mechanisms for positioning cells prior to lysis, which is a disadvantage for a rapid lysis device. In fact, the cell positioning time is much higher than cell lysis time.

5. The number of cells available in invasive or cultured samples, such as blood, are quite large $\left(5 \times 10^{6}\right.$ per $\left.\mu \mathrm{l}\right)$ [96]. For non-invasive samples, however, number of cells are very few (in the range of $10^{3}$ cells per sample).

\subsection{Research Approach}

In this section, the approach followed in this research to develop the proposed device and method are described.

\subsubsection{Concept development}

The concept formulation was aimed to address the challenges to realize a rapid and portable cell lysis device. In each iteration of the device development, the acquired knowledge from previous stages were considered and new or improved features were implemented.

\subsubsection{Choice of fabrication technology}

All reported devices in the literature for EL and EP were prepared using custom fabrication process $[64-66,82-87,91,94,97-100]$. Many of these custom fabrications were based on glass substrate using chemical etching [82, 87, 91, 97], depositing Polydimethylsiloxane (PDMS) $[65,66,83,85]$, or photoresist (such as SU-8) [84, 
86]. Some fabrications were reported using bulk etching of Si-substrate [98-100] or Polymethylme-thyl Acrylate (PMMA) substrate [94]. Devices were also made through modification of existing laboratory equipment [64].

For our device development, we preferred to use commercially available fabrication processes. The choice was based on the following two factors:

1) Limited or unavailability of a fabrication facility with the required resources for such device development.

2) The device would be easily prototyped and tested at a low cost.

The latter was an important consideration of this research as cell lysis step was a part of several steps in a DNA detection process that needed to be integrated with the developed device at a future stage. For such process integration, all components of the microfluidic system need to be amenable to a well established fabrication technology for compatibility [27].

For fabrication of the devices, we have considered various commercially available fabrication technologies. Technologies suited for this application were Protolyne Microfluidic Device (MFD), Low Temperature Co-fired Ceramic (LTCC), Metal MultiUser MEMS Processes (MetalMUMPs), and Sensonit technology (from 2007 onwards). For the application involved, important criteria included optical transparency, minimum electrode distance, maximum channel depth and foundry support through Canadian Microelectronics Corporation (CMC). Optically transparent device would allow observation during EP and EL process. Smaller electrode distance was preferable as it would allow lower applied voltage to develop sufficient electric field for EL. A minimum channel depth of $20 \mu \mathrm{m}$ was required so that cells can move through the channels. Foundry support availability through CMC was preferred for a low cost solution. From this context, a brief comparison of these technologies in the context of our research interest is presented in Table 1.1. These technologies are briefly outlined below.

Protolyne microfluidic device: In this microfluidics technology, two glass slides 


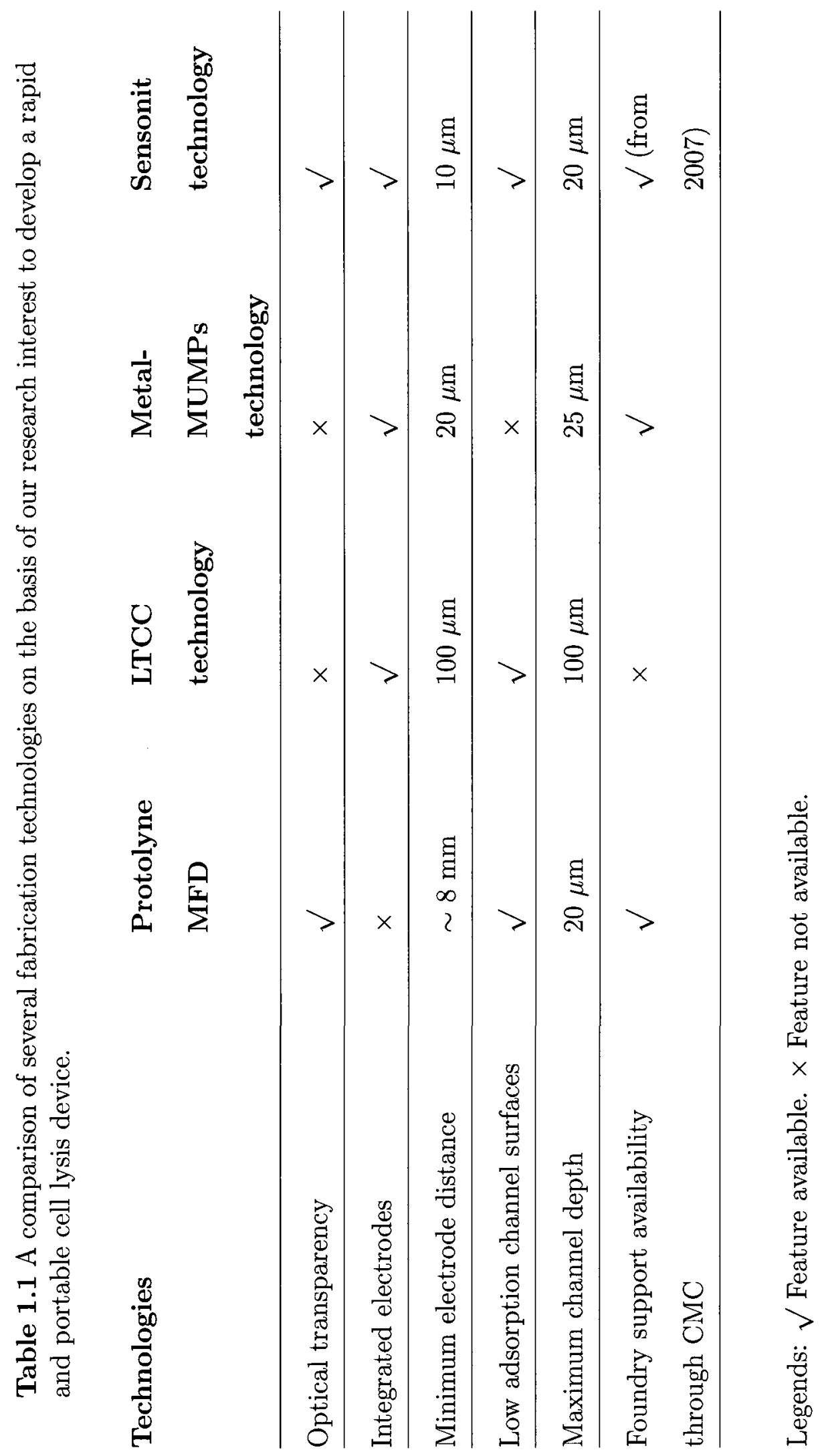


are fused together where the bottom slide contains microchannels formed by chemical etching process, and the top slide contains predefined access holes. The fabrication technology is offered by the foundry Micralyne Inc., Canada [www.micralyne.com] and available through CMC since early 2000.

Low Temperature Co-fired Ceramic (LTCC): Developed for packaging of devices, the LTCC technology composes of thin layers of ceramics trimmed to form slits and cuts. These single sheets are laminated together and heated (fired) at about $850^{\circ} \mathrm{C}$ to join them together. The foundry that offers this technology is IMST GmbH, Germany [www.ltcc.de]. This technology is available since 2000 from IMST GmbH, Kamp-Lintfort, Germany. This technology is not yet available through CMC.

Metal Multi-User MEMS Processes: Among several Multi-User MEMS Processes (MUMPs) technology, MetalMUMPs was the best suited for biomedical applications, primarily due to its deeper $(25 \mu \mathrm{m})$ trench and availability of a metal electrode layer. This process contains a high aspect ratio electroplated nickel surface micromachining process. PolySi and metal layers (Cr-Pt-Cu-Ni-Au) are deposited on a Si-substrate before bulk etching of the Si substrate to form trenches, which can act as channels and reservoirs. The process is offered by MEMSCAP Inc., USA [www.memscap.com] and available through CMC since mid 2000 .

Sensonit technology: Sensonit technology can be used to create microchannels with integrated electrodes on a glass substrate. Here, two glass slides were fused together, where the bottom slide contained in-channel electrodes and bottom side of the top slide contained channels and access holes. This fabrication was available through Micronit Microfluidics BV, The Netherlands, and offered through CMC since December 2007 [www.micronit.com].

After considering the factors presented in Table 1.1, we fabricated our first (initial) 
device using Protolyne MFD as a preliminary study. Our second (intermediate) device was fabricated using MetalMUMPs technology (prior to December 2007) and the third (final) device was fabricated using Sensonit technology as it became available after December 2007.

\subsubsection{Device design and modelling}

The device design, modelling and analysis required various software and Computer Aided Design (CAD) tools. For layout design of these devices, L-edit, Cadence Virtuoso and MEMSPro v6 tools were used. For modelling and analysis, Finite Element Method (FEM) tools such as Maxwell3D tool (Ansoft Corp.) and Comsol Multiphysics (Comsol Inc.) were used. Visual Basic (VB) script files were used within Maxwell3D tool for automated model generation of various dimensions, simulation and data extraction. Verilog coding was used to program Altera DE2 FPGA board using Altera Quatrus software (Altera Corp., CA, USA) and ModelSim (Mentor Graphics, OR, USA). Analog circuit simulations were conducted using Cadence (Cadence Design Systems, CA, USA) and LTSpice IV (Linear Technology Corp.) tools.

\subsubsection{Testing and evaluation}

Custom platforms were built to develop testing fixtures of the devices during experiments. Various stains were used in different experimental phase, including Hematoxylin, Eosin and Alexa Fluor 488 Annexin V. Different buffer mediums were tested including Dulbecco's Phosphate Buffered Saline (D-PBS), Deionized Water $\left(\mathrm{D}-\mathrm{H}_{2} \mathrm{O}\right)$, and hydrophilic, hydrophobic, isotopic solutions. Picture sequences were taken using a Charge-Coupled Device (CCD) camera connected with an optical microscope using either $10 \times, 40 \times$ or $100 \times$ lenses. Fluorescence images were captured using an Olympus Fluorview microscope. A high voltage electrophoresis power supply was used for exciting the Protolyne MFD, Altera DE2 FPGA board with output circuitry was used for the MetalMUMPs device and a signal generator was used for the Sensonit 
device. An oscilloscope (Tektronix Inc.) was used to monitor and record excitation pulses. Testing and evaluation were performed in labs of Medical Devices Center or Department of Electronics.

\subsubsection{Data analysis}

For data extraction, analysis, and plotting, several Matlab programs were written. Some of the trivial plotting used Microsoft Excel. For image processing and plotting of extracted data of the number of stained pixels in bitmap image sequences obtained from the experiments of the final device, codes were developed using VB (Microsoft Corp, CA, USA) and Matlab (Mathworks Inc., MA, USA) tools. For image editing, labelling, and enhancement, ImageJ (National Institute of Health, USA), Irfanview (Freeware) and Inkscape (Open Source Scalable Vector Graphics Editor) tools were used.

\subsection{Summary of Results}

This section outlines the key findings and important results, key attributes of the final device and method, as well as contributions and achievements that stemmed from this research.

\subsubsection{Key findings and important results}

Followings are the key findings and important results from our experiments with all the developed devices.

\section{The initial device:}

1. Results demonstrated that EL of human cells occur with the application of a high electric field sustained for about $7 \pm 2$ seconds. 
2. Applied voltage required was 750 to $900 \mathrm{~V}$ supplied using a high voltage power supply unit.

3. Long microchannel introduced issues like cell attachment, clogging, cell positioning, etc.

4. Estimation of the electric field using simulation suggested that the microchannel model of parallel plate structure provides over $90 \%$ accuracy of electric field as long as the microchannel length is longer than $2.3 \mathrm{~mm}$.

5. For smaller dimensions of microchannel with reservoir configuration, the analytical model produces over $10 \%$ error, and corrective extension is proposed in this research. However ideal microchannel configuration was not subjected to such error.

\section{The intermediate device:}

1. Fluidic encapsulation was a challenging issue with such device and a simple procedure was developed to resolve the issue using polyimide and common laboratory materials.

2. Cells were observed to deform with the application of $20 \mathrm{~V}$ supply using pulses of $1 \mathrm{~ms}$ or more width.

3. Larger pulse widths hindered observation due to numerous bubble formation.

\section{The final device:}

1. Simulation results suggested that electric field inside the microchannel is sufficiently high with the application of $20 \mathrm{~V}$ to enable cell lysis inside microchannels.

2. A protocol was developed to stain freely flowing cells (not attached to any surface) by replacing the conventional washing step with centrifuge steps. 
3. EL process releasing DNA was observed with pulses of $20 \mathrm{~V}$ supply for 5 seconds or more using the device. The developed electric field was estimated to about $2 \mathrm{MV} / \mathrm{m}$. The corresponding power consumption was as low as $0.4 \mathrm{~W}$.

4. EP were detected (through reduction of stained pixels) by applying smaller voltages $(5$ to $20 \mathrm{~V})$ with shorter duration pulses $(10 \mu$ s to $100 \mathrm{~ms})$. The corresponding electric field were about 500 to $2000 \mathrm{kV} / \mathrm{m}$.

5. Simulation result showed that the temperature rise in the cell lysis chamber of the final micro-device was less than $2.2^{\circ} \mathrm{C}$ determined using $\mathrm{FEM}$ simulations.

6. Existing electric circuit cell model was not able to model electroporation effectively, as pore formation dynamics was not considered in the model. Improvement is proposed by incorporating a pore model and simulation results were justified using corresponding experimental results.

7. The longer pulses and higher magnitude of pulses resulted faster decrease of stain, indicating higher Degree of EP. However, cells were deformed for relatively longer pulses.

8. A single pulse was more effective to cause morphological change and a higher electroporation fluidic exchange compared to multiple pulses delivering the same total excitation time.

\subsubsection{Distinguishing attributes of the final device}

Key distinguishing features of the final device for being the most effective device for $\mathrm{EP}$ and EL compared to previously reported devices are:

1. Interdigitated configuration of integrated electrodes.

2. Electrodes laid at the bottom of the microchannels.

3. Cell loading ports were directly above the cell lysis chamber. 
These key features achieved the following advantages:

1) Cell size: As the electrodes in the device were on the bottom of the microchannel, microchannel width or height was not restricted by the electrode gaps. This electrode configuration created higher electric field at the bottom of the microchannel, where the cells settle. This unique feature of electrode configuration compared to other reported devices allowed us to have smaller electrode gaps compared to cell diameter, a feature that was not feasible in any other reported devices.

2) Electric field coverage: The electrodes in the final device were configured in inter-digitized configuration without pointed edges. The configuration facilitates a high electric field in the cell lysis chamber between electrodes that occupies $50 \%$ of the chamber area.

3) Cell positioning time: In this device, a high electric field was developed simultaneously among all electrode gaps within the cell lysis chamber, so that any part of a cell within the gap will experience the high electric field and will be lysed. This feature enabled cells to be lysed at all location of the lysis chamber without aligning them along the pointed edges as required in the other reported devices. Hence, there was no time delay associated for cell positing compared to other reported devices.

4) Fast sample introduction ability: The samples in the final device were introduced through the loading ports directly above the microchannels. This leaded to a shorter sample load time compared to other devices where cells were introduced at the end of microchannels and needed to be transported to the cell lysis region.

5) Reusability: Having the loading ports above the $400 \mu \mathrm{m}$ wide microchannels allowed reliable cleaning of the microchannels with less possibility of clogging by cell debris, thus allowed reuse of the device.

6) Power supply requirement: The device operating procedure required only one low voltage power supply $(20 \mathrm{~V})$ of pulsed type compared to multiple power supply requirement in some other cases. This resulted in a simpler system with lower cost, as well as a simpler operating protocol. 


\subsubsection{Contributions}

Following major contributions were made through this research to enhance the current scientific knowledge.

1. Development of a rapid and portable device for electrical lysis and demonstration of DNA release from nucleus: The research resulted development of a small, effective, portable micro-device. With this device, we have demonstrated breakdown (lysis) of nucleus to release DNA with electric fields. The device produced optimal results due to effective design features, like interdigitated electrodes to perform EL and EP with a low applied voltage and within a small amount of time, and minimum loading time achieved by sample loading ports above the lysis chamber. EP of cells in various excitation parameters were investigated and compared. In addition, analysis of electric field and temperature of the microchannel were conducted. The developed device clearly demonstrated the ability to release DNA from sample cells that indicated breakdown of nucleus, not previously demonstrated. This is significant advancement to access DNA from cell towards rapid and portable DNA detection device.

2. Improvement of the current cell model: An improvement over the current cell model for electroporation analysis has been developed by incorporating a pore model to address pore dynamics within the current electric circuit model of a cell under an electric field. The improvement enabled us to define two key quantification terms for electroporation:

a) Degree of Electroporation: The ratio of the total pore area that is formed during electroporation to the surface area of the cell, representing a metric for severity of electroporation.

b) Electroporation Fluid Exchange Parameter (EFEP): The product of Degree of Electroporation and the duration of electroporation representing a metric for fluidic exchange during electroporation. 
Other contributions resulted from these research include:

1. Staining protocol of freely flowing cells: Development of a protocol for staining of freely flowing cells (unattached to any surface), where conventional washing step was replaced by centrifuge steps.

2. Fluidic encapsulation protocol for BioMEMS device: A protocol for fluidic encapsulation of BioMEMS devices was developed that uses polyimide and several common lab components.

\section{Error minimizing extension of the mathematical model of electric} fields inside microchannels: Electric field development inside microchannels were analyzed using a simple mathematical model and simulated using FEM simulation tool for various microchannel dimensions and excitation voltages. An error minimizing extension of the simple mathematical models to calculate electric fields inside microchannels of a MFD was proposed to reduce estimation errors using the mathematical model.

\subsubsection{Achievements}

Following publications resulted from this research:

(1) B. I. Morshed, M. Shams, T. Mussivand, "An Analysis of Electric Fields Developed Inside Microchannels of Microfluidic Devices", IEEE Intl. Conf. Electrical and Computer Engineering, pp. 261-265, Dec. 2008.

(2) B. I. Morshed, M. Shams, T. Mussivand, "A Microfluidic device to lyse cell membrane using electric field", Canadian Workshop on MEMS and Microfluidics, Aug. 2007.

(3) B. I. Morshed, M. Shams, T. Mussivand, "Development of a rapid cell membrane lysing device using electrical pulses", 16th World Congress of the World Society of Cardio-Thoracic Surgeons, p. 172, Aug. 2006. 
A patent titled "Micro-device for Cell Membrane Electroporation and Lysis" was submitted to counsel for filing in October 2009. As the patent is in process at present, the results are not published yet.

Following journal paper drafts are ready for submission after patent filing.

(1) B. I. Morshed, M. Shams, T. Mussivand, "Review of Recent Advancements on Electrical Lysis", 13 pages.

(1) B. I. Morshed, M. Shams, T. Mussivand, "Electrical Lysis of Fingerprint Cells in a Microchannel-based Microfluidic Device", 12 pages.

(3) B. I. Morshed, M. Shams, T. Mussivand, "Effective Modelling of Electroporation Dynamics", 9 pages.

(4) B. I. Morshed, M. Shams, T. Mussivand, "Microdevice for Electrical Lysis and Electroporatoin", 11 pages.

\subsection{Organization}

This dissertation is organized as follows. Chapter 2 discusses literature review relevant to EP and EL. Chapter 3 discusses the design features of the developed devices. In Chapter 4 , simulation results of the devices are described. Experimental findings are presented in Chapter 5. Chapter 6 delivers contribution to dynamics and modelling by a proposed pore model to be included with the current cell model for effective modelling of EP dynamics. Chapter 7 concludes the dissertation by summarizing key results and providing future directions. 


\section{Chapter 2}

\section{Literature Review}

In this chapter, a comprehensive literature review is provided to discuss the dynamics of Electroporation (EP) and Electrical Lysis (EL), recent advancements, significant experimental findings, modelling approaches and limitations. A brief review of relevant cellular biology is provided at the beginning to provide background information.

\subsection{Cell Biology}

This section briefly discusses cellular biology relevant EP and EL.

\subsubsection{Composition of the cell membrane}

The cell membrane, a 4 to $8 \mathrm{~nm}$ thick bilayer lipid membrane, is a continuous sac that surrounds the cell and acts as a physical barrier between the intracellular and the extracellular environment [58]. The cell membrane is mainly composed of phospholipids (more than $50 \%$ of the total membrane lipid mass). It has a hydrophilic (having an affinity for water) polar head containing a phosphate group and two hydrophobic (lacking affinity for water) long tails made of hydrogen chains. A glycerol or sphingosine connects the head and the tails of the phospholipid molecule. As both intracellular and extracellular mediums are primarily hydrous fluids, the hydrophobic 
tail spontaneously arranges to shield itself from these fluids in a two-layer formation, known as bilayer lipid membrane (BLM). Besides phospholipids, glycolipids and steroids are found in cell membrane. Embedded in the cell membrane are integral proteins, peripheral proteins, carbohydrates, receptors, cholesterol etc.

There are several kinds of phospholipids found in the cell membrane, such as phosphatidylcholine (PC), phosphatidylethanolamine (PE), phosphatidylserine (PS), and phosphatidylinositol (PI) [58]. Among these, 1,2-dimyristoyl-sn-glycero-3- phosphorylcholine (DMPC) is the most extensively found phospholipid molecule in the cell membrane. Chemical compositions of this phospholipid molecule is given in Figure 2.1(a). Symbolic representation of a phospholipid molecule and a cross-section view of a BLM formation is schematically shown in Figure 2.1(b) and 2.1(c), respectively. This cell membrane must be ruptured to access internal contents of a cell. This process of disintegrating the cellular membrane to release the cell contents is known as cell lysis $[11,26]$.

\subsubsection{Nucleus of a cell}

Among various organelles found inside a cell, the nucleus contains Deoxyribonucleic Acid (DNA) that carries hereditary information. The nucleus is a double membraneenclosed organelle that controls most of the activities of the cell [11]. The DNA inside nucleus resides in the form of chromosomes, which are organized structures of DNA and proteins (named histones) in a coiled form. A single chromosome contains many genes, regulatory elements and other genetic sequences, formed by arrangements of four types of nucleotides: Adenosine (A), Guanine (G), Cytosine (C) and Thiamine (T) [22]. The nucleotides are arranged in a double-helix structure. A diploid human cell nucleus contains 22 pairs of homologous non-sex chromosomes (called autosomes) and 2 sex chromosomes [10]. 


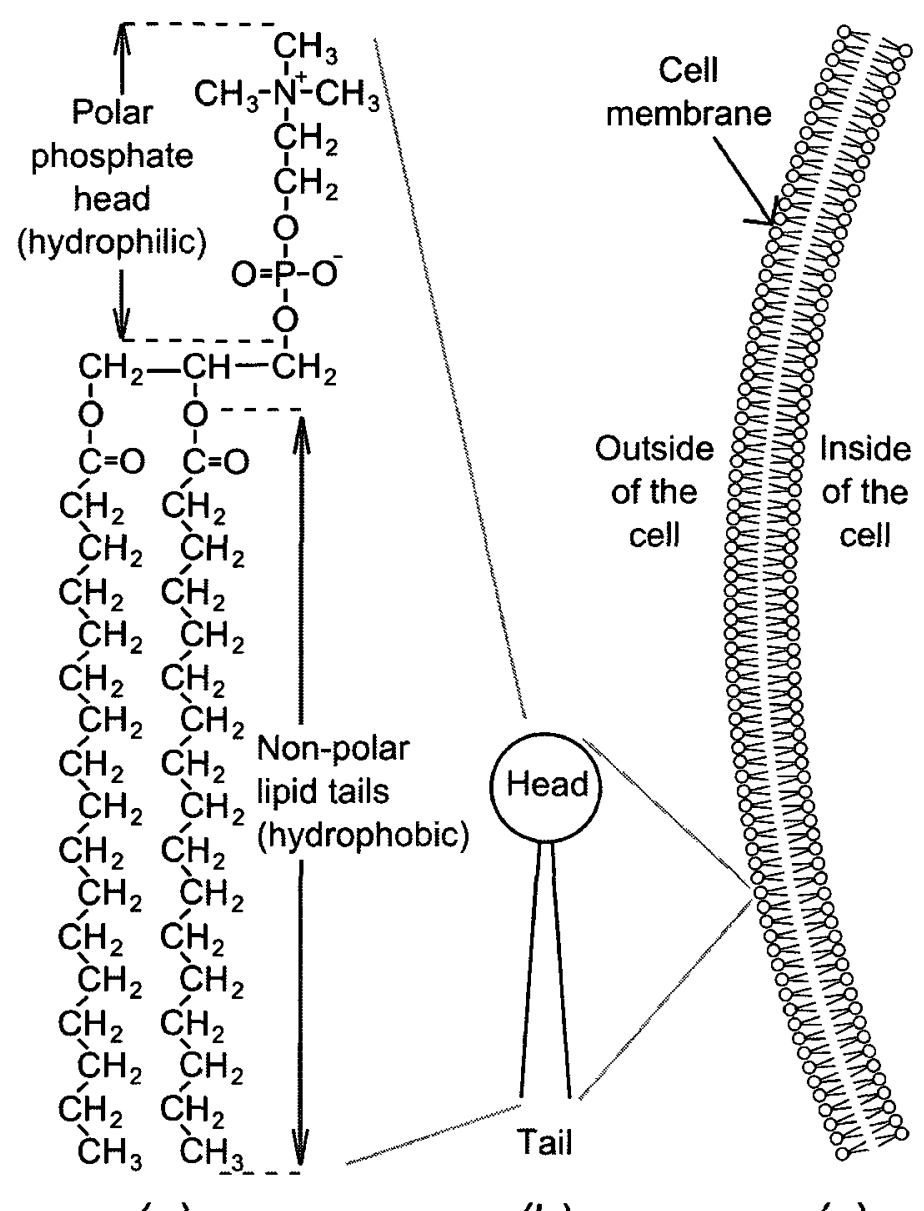

(a)

(b)

(c)

Figure 2.1 (a) Chemical composition of a DMPC molecule - a phospholipid molecule extensively found in cell membranes. (b) Symbolic representation of a phospholipid molecule. (c) A schematic representation of a segment of a cell membrane composed of phospholipid molecules arranged in a BLM formation. 


\subsection{Dynamics of Electroporation and Electrical Ly- sis}

In this section, dynamics involved in EP and EL is discussed. Two properties of phospholipid bilayer of a cell are susceptible to an external electric field $[1,101]$ :

1. Electric dipoles of phospholipids: The phosphate group (head) is electropositive in nature and the hydrogen chains (tails) are electronegative. Hence, each phospholipid molecule acts as an electric dipole when placed inside an external electric field. These phospholipids undergo reorientation when the electric field is very high. The reorientation causes formation of new pores through the cell membrane.

2. Permeability of the membrane: The bilayer membrane has a very small, but finite, electrical permeability. In the presence of a high electric field, a small amount of ions flow through this membrane, resulting in Joule heating and thermal phase transition of cell membrane [1].

As the generated Joule heat during thermal phase transition due to the permeability property of the membrane might damage the contents of the cell, usually current flow is kept very low. The dipole property of phospholipid molecules, on the other hand, is exploited for EP or EL.

\subsubsection{Development of transmembrane potential and forma- tion of pores}

Dynamics involved in pore formation within the cell membrane is still a topic of discussion $[1,60]$. The commonly acceptable hypothesis is the accumulation of charges on both sides of a cell membrane causing reorientation of phospholipid molecules $[1,2,82,102-104]$. According to this hypothesis, when a cell suspended in an ionic fluid is excited by an electric field, a large amount of charges accumulate along the cell 
membrane, inducing a "transmembrane potential" across the cell membrane [1]. The transmembrane potential is much larger than the action (or gating) potential, which is a living cell (neuron and muscle) characteristic of spontaneous electrical discharge of 70 to $90 \mathrm{mV}$ along the cell membrane [11]. The transmembrane potential causes an electrical force on phospholipids, acting as electric dipoles, resulting in a stress

for reorientation of these phospholipid molecules $[2,102,103]$. The transmembrane potential and the electrical force are proportional to the electric field strength and to the square of the electric field strength, respectively [1].

If the applied electric field is increased such that the transmembrane potential reaches a critical value of $1 \mathrm{~V}$, the phospholipid molecules undergo conformal change in the membrane structure. This causes formation of a large number of hydrophilic pores through the cell membrane, especially where the cell membrane is perpendicular to the electric field lines $[1,60]$. The electric field required to initiate pore formation, known as the "threshold electric field", has a typical range of 0.1 to $0.15 \mathrm{MV} / \mathrm{m}$ for erythrocytes, protoplasts and spheroplasts [1]. The time interval between application of the electric field and initiation of pores is termed as "nucleation period" [104]. These pores expand with time if the electric field is not withdrawn, resulting in phase transitions in the cell membrane [102].

Depending on the duration of the applied electric field, the pores formed in the cell membrane can stay reversible or become irreversible. The first case results EP and the second case results EL.

\subsubsection{Reversible electroporation phenomenon}

If the electric field is sustained, the average pore-size is increased, but if the electric field is withdrawn while the pore diameter is below a critical range, annealing and resealing takes place due to molecular interactions, resulting in a reversible system $[104,105]$. This phenomenon of reversible pore formation in the cell membrane is called electroporation (EP) $[1,60,82,98]$. EP represents a cycle of structural rear- 
rangements, where the intermediate states of the unidirectional annealing process are probably different from those of the unidirectional pore formation process in the presence of the external electric field [102]. The time required for the annealing process to be completed is called "relaxation time" and the transition path difference in pore formation and annealing process is known as "electroporation hysteresis" $[1,106]$.

\subsubsection{Irreversible electrical lysis phenomenon}

If the threshold electric field is maintained for a critical time duration, the pores formed during EP expand in diameter and reach a critical pore diameter (about $40 \mathrm{~nm}[104,105])$. Beyond this pore diameter, pores expand spontaneously in an uncontrolled fashion. Thus the cell membrane structure can be completely destroyed, exposing the internal organelles to the carrying medium. Thus cell lysis (or membrane disintegration) is achieved using a high electric field and the process is known as electrical lysis (EL), electro-lysing or electric field lysing [64,65, 77, 82, 83, 91, 94, 97$99,107,108]$. The induced membrane potential to initiate EL is known as the critical transmembrane potential and the corresponding time required is known as the critical time duration.

\subsection{Studies of electroporation and electrical lysis}

The history of EP and EL study dates back to 1967, when Pliquett observed that electric field developed with an Alternating Current (AC) excitation affects the membrane of Oxitrichide single cells [1]. Later, Sale and Hamilton observed that short electric pulses, above a critical field strength, cause lysis of bacterial propoplasts and spheroplasts, and erythrocytes $[67,68]$. These phenomena have since been studied to understand relevant dynamics [64,85,101], and the knowledge has been applied in various biotechnology, therapeutic, genetics and biomedical engineering activity $[61,63,82,84,86,88,94,97,109-111]$. Advancement in microfabrication tech- 
nology, such as Biomedical Microelectromechanical System (BioMEMS), Lab-on-achip (LOC) and microfluidics, have facilitated this research $[28,112]$. Major studies of these phenomena are discussed in this section.

\subsubsection{Recent advancements in electroporation}

EP is usually achieved through Pulsed Electric Field (PEF) technique, where pulse durations are small enough so that pores do not reach critical pore dimensions [84, 98-100, 107, 113, 114]. Such short-duration pulses produce negligible joule heating, hence cell survival rates are increased.

The degree of membrane electropermeabilization (permeability of cells to nonionic molecules due to EP), molecular uptake and cell survival rate upon EP depend critically, among other parameters, on the duration of the applied electric field pulses [60]. If the electric field strength is sufficiently strong, shorter pulses (duration of microsecond or less) are more suitable for EP, whereas longer pulses (duration of tens of microseconds or more) result in EL. Experimentally, it is shown that increased duration of pulses result in an exponentially decaying threshold electric field for EP [106, 115]. This dependency is known as the strength-duration relationship [1].

Schoenbach's research group observed that larger pulses $(>2 \mu \mathrm{s})$ damages the cell membrane, whereas smaller pulses ( $<50 \mathrm{~ns})$ do not damage the cell membrane; but in both cases, cells eventually die when the electric field of sufficient strength is applied [4,114]. For instance, with $5 \mu \mathrm{s}$ pulses, the outer membrane is damaged instantly, whereas with $50 \mathrm{~ns}$ pulses, the outer membrane remains intact. However, $90 \%$ of the exposed cells die after 3 days, while the control group increased by a factor of two. If the electric pulse duration is reduced below nanosecond range, then internal organelle membranes of a cell are disintegrated, while the cell membrane remains intact $[4,105,115]$. These nanosecond duration (or ultrashort) pulses damage membranes of internal organelles, rather than the cell membrane [4]. Besides, the required electric field for cell membrane poration is higher with shorter pulses 
compared to longer pulses. For example, 10 ns pulses with $6.5 \mathrm{MV} / \mathrm{m}$ electric field showed no indications of poration of the cell surface membranes, albeit, 60 ns 2.6 $\mathrm{MV} / \mathrm{m}$ pulses showed dye uptake indicating electroporation through surface membranes [4,114]. Ultrashort pulse treatment with a high electric field of $E$. coli cells have shown that both cell mortality and energy consumption are higher for longer pulses ( $>20 \mu \mathrm{s}$ ) than shorter pulses (60 and $300 \mathrm{ns)} \mathrm{[109],} \mathrm{because} \mathrm{the} \mathrm{threshold} \mathrm{field}$ increases with reduced pulse duration which consumes less energy. For example, the threshold electric field increases from $0.72 \mathrm{MV} / \mathrm{m}$ for $36 \mu$ s pulse duration to $4 \mathrm{MV} / \mathrm{m}$ for 60 ns pulse for E. coli cells [115].

Huang and Rubinsky performed EP experiments with a microfabricated device consisting of two chambers connected through a micro-hole [99]. The distance between the electrodes was approximately $900 \mu \mathrm{m}$ and the diameter of the hole was about 5 $\mu \mathrm{m}$. For EP, 10 to $15 \mathrm{~V}$ pulses were applied with a duration of $100 \mathrm{~ms}[98,100]$. Human prostate adenocarcinoma cells (ND-1 cell line) were used to load YOYO-1 dye, which showed $100 \%$ EP of trapped cells in the micro-hole. Successful expression of inserted EGFP genes in the cell were performed by genetic manipulation on single ND-1 cell inside the chip.

Effect of medium conductivity on EP is not established yet. As medium conductivity in increased, higher EP is observed in Ch. reinh. (green algae cells) using dye uptake [106], or E. Coli determined by survivality [109]. Survival rate of E. coli reduced from $90 \%$ to $30 \%$ with a 10 fold increase in resistivity [62]. It is shown that when the medium outside the cell has a lower conductivity, the developed transmembrane potential will be less due to membrane conductance, surface admittance and space charge effect [116]. However, experimental results from some researchers suggest the reverse. For instance, electroporation of myeloma cells using 10 to $100 \mathrm{nsec}$ pulses of $15 \mathrm{MV} / \mathrm{m}$ electric field showed that cell stain update increased markedly with decreasing conductivity of the surrounding medium [60].

When AC electropermeabilization pulses for ellipsoid cells is used, higher EP efficiency is observed for shorter diameter of cells, as well as for higher frequency and 
shorter pulse duration [61]. Curvature of cell membrane at maximum transmembrane potential seems to play an important role, as shorter axis of ellipsoid cells resulted higher electropermeabilization efficiency compared to longer axis. Cell orientation might contribute to the fact that the voltage at the onset of EP can be different by as much as $20 \%$ of the applied voltage in different cells of the same cell-type [99].

A microfabricated parallel channel EP device using an ac excitation was studied by Jensen et al [84]. Continuous flow variable width microchannel based electroporation device was investigated by Wang and $\mathrm{Lu}$, where different cell swelling rates were reported after electroporation for various osmolarity of the medium [85].

\subsubsection{Recent advancements in electrical lysis}

The required electric field strength for EP is a function of pulse duration. For example, $50 \mathrm{kV} / \mathrm{m}$ pulses with $6 \mathrm{~ms}$ duration were found to be sufficient to lyse Jurkat cells (cell lines used for cancer study). But the cell membrane of the same type of sample is found to be intact with $6 \mathrm{MV} / \mathrm{m}$ ultrashort pulses with $60 \mathrm{~ns}$ duration, even though cells eventually died, because of severe damage to internal organelles [4]. Survivability of treated cells with PEF depends on pulse width [109,112]. Above a minimum threshold electric field strength, pulses with wider width causes greater death rate to cells than with shorter width. Leukocytes or White Blood Cell (WBC) (1:100 dilution) from blood samples were lysed using $10 \mu$ s pulses of $50 \mathrm{MV} / \mathrm{m}$ [86].

Lysis time of less than $33 \mathrm{~ms}$ or less than $66 \mathrm{~ms}$ were reported using $2 \mathrm{MV} / \mathrm{m}$ electric field with pulse width of $1 \mathrm{~ms}$, whereas lysis time increases more than $1 \mathrm{~s}$ with pulses of $100 \mu \mathrm{s}$ for the same electric field [64,88]. Similarly, electrical lysis is observed with $35 \mathrm{~V} 100 \mathrm{~ms}$ pulses, whereas only electroporation is observed with 15 $\mathrm{V} 100 \mathrm{~ms}$ pulses, using a microfabricated device with a microhole [98].

For the case of AC excitations, cells treated with lower frequency and applied voltage have more permeable membranes, where cells treated with higher applied voltages are more likely to have undergone complete lysis [84]. With AC excitations, 
cells undergo dielectrophoretic force in addition to any electrical lysis force. This dielectrophoretic force is responsible for the stretching of cells between electrodes, which might contribute to cell lysis [82].

For the case of direct current (DC) excitations, heat generation is a concern. Favorably, large surface area to volume ratios associated with microfluidic devices limit the heating effect to less than $1 \mathrm{~K}$ [84]. Examples of such excitation for EL are erythrocytes collected from human blood samples that were lysed within $40 \mathrm{~ms}$ with applied voltages of $1.4 \mathrm{kV}$ and $800 \mathrm{~V}$ supplies within the working electrolyte (pH 9.2) [97]. In another study, Acute Myloid Leukemia (AML) cells were lysed with $1380 \mathrm{~V}$ across $4.2 \mathrm{~cm}$ long microchannel in less than $300 \mathrm{~ms}$ [94].

Channel geometry can be manipulated to amplify electric field in a certain section of the channel for EL $[65,66,85,99,100]$. In this case, the required applied voltage to develop the threshold electric field is lower than that needed by a channel without geometric modification, in addition to reducing Joule heating $[65,66]$. Variable width microchannels, creating concentrated electric field along the channel, was used to lyse chinese hamster ovary (CHO-K1) cells with $0.1 \mathrm{MV} / \mathrm{m}$ or higher electric field $[65,85]$. A similar device was used to lyse Red Blood Cell (RBC) in continuous flow mode, where a bias voltage of $50 \mathrm{~V}$ created $0.12 \mathrm{MV} / \mathrm{m}$ in a narrow section of the microchannel that was sufficient for EL [83]. Concentrating EL using a micro-hole reduced excitation voltage for the breakdown of human prostate adenocarcinoma cells to $34 \mathrm{~V}$ using $100 \mathrm{~ms}$ pulse [98,99]. Osmotic pressure might influence lysis using such technique due the pressure difference across the micro-hole structure.

EL process initiation can sometimes result in cell fusion, vesicle formation, and electromechanical rupture [102]. A variety of cell membrane behaviors are noted after treatment, such as swelling, morphological changes (membrane extrusion in particular) and rare electro-fusion of two cells [84]. Tertiary effects of EL are temperature increase due to dissipative process and interactions in electrode-electrolyte interface, such as ion release, $\mathrm{H}_{2}$ and $\mathrm{O}_{2}$ formation [102].

Yeast (S. cerevisiae) protoplast and $E$. coli cell lysis were reported at $1 \mathrm{MV} / \mathrm{m}$ 
with pulse width of $100 \mu \mathrm{s}$ and $0.7 \mathrm{MV} / \mathrm{m}$ with pulse width of $500 \mu \mathrm{s}$, respectively [82]. Jurkat line of human leukemia cells were lysed using electric field strengths of 15 to 50 $\mathrm{kV} / \mathrm{m}$ peak-to-peak square wave $50 \mathrm{~Hz}$ AC fields with 30 to $40 \mathrm{kV} / \mathrm{m}$ DC offset [91]. Human HT-29 cells were lysed under an electric field of a few $100 \mathrm{~V} / \mathrm{m}$ [77]. This shows that the electric field required for cellular breakdown is largely dependent on the cell type. Cell swelling rates during electrical lysis process are reported to depend on buffer type, too. For instance, average swelling of cells in hypotonic buffer was $175 \%$, in isotonic buffer $155 \%$ and in hypertonic buffer $160 \%$ with $50 \mathrm{kV} / \mathrm{m}$ in $300 \mathrm{~ms}$ [85].

A probe-based electrical lysis device described in [64] reported lysis time of less than $33 \mathrm{~ms}$ with $40 \mathrm{~V}$ pulses of $1 \mathrm{~ms}$ width for a probe distance of $20 \mu \mathrm{m}$ creating an electric field strength of $2 \mathrm{MV} / \mathrm{m}$. SWLA-2 murine hybridoma (HB12560) cells were lysed with electric field of $46 \mathrm{kV} / \mathrm{m}$ with $500 \mathrm{~ms}$ pulses, whereas field strength of 27 $\mathrm{kV} / \mathrm{m}$ was not effective [113]. Munce et al. reported EL in less than $300 \mathrm{~ms}$ [94]. Hence critical time might also be dependant on the cell type.

Recently electrochemical cell lysis was demonstrated with a small voltage as $5 \mathrm{~V}$, with required time for lysis reported as 5 minutes [93]. The method, however, did not achieve significant time advantage compared to chemical cell lysis.

\subsubsection{Chronological list of major findings}

A chronological list of major findings relevant to EP and EL are tabulated in Table 2.1 .

\subsection{Modelling of Electroporation}

A biological cell in an electric field can be modelled for engineering analysis using various approaches, such as mathematical model using Laplace equation for the potential distribution and energy function $[1,60,82,86,102,106,117]$, equivalent electric circuit model $[4,105,109,110,114,115,120]$, molecular dynamics approach $[2,3,104,121-123]$, 


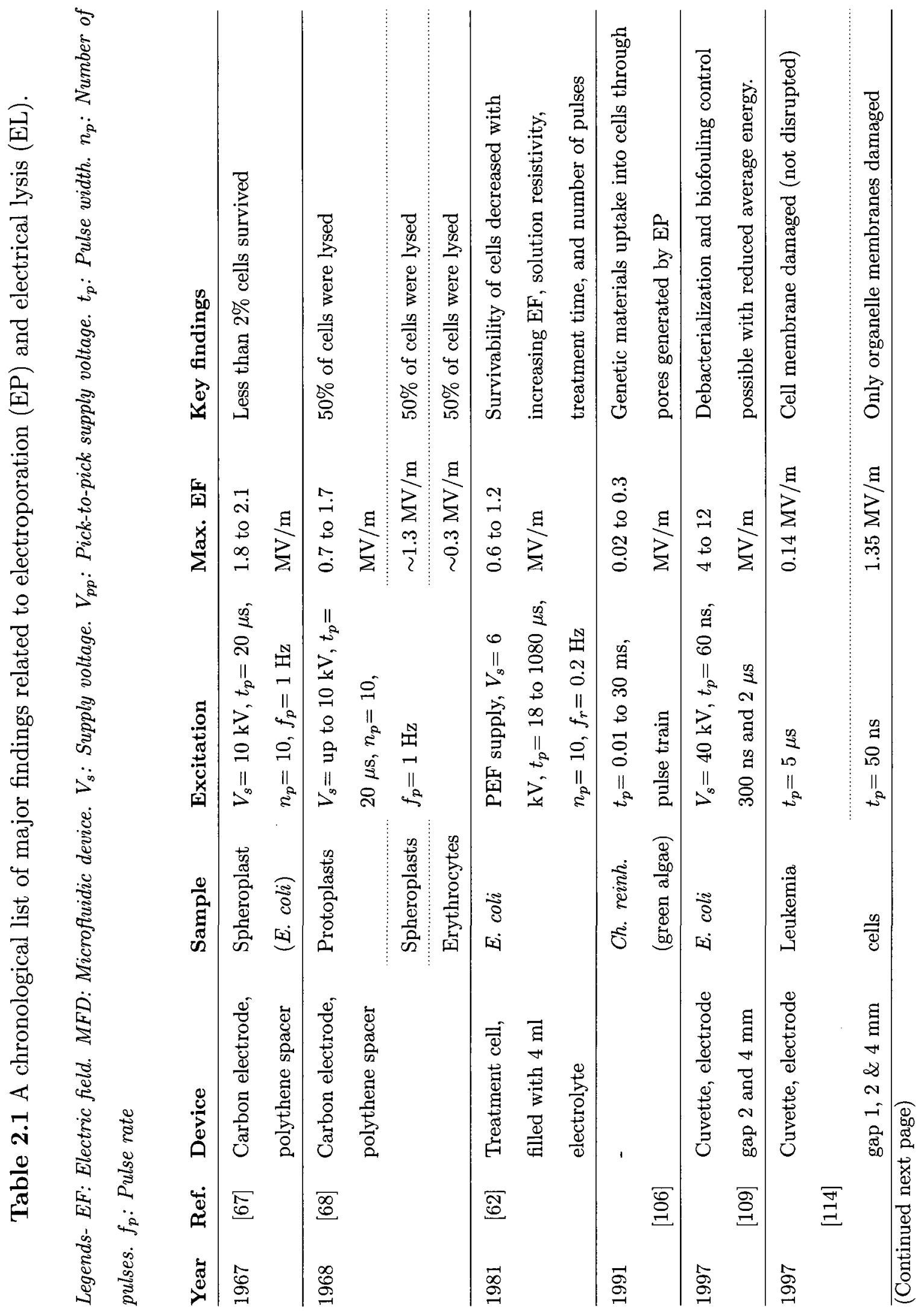




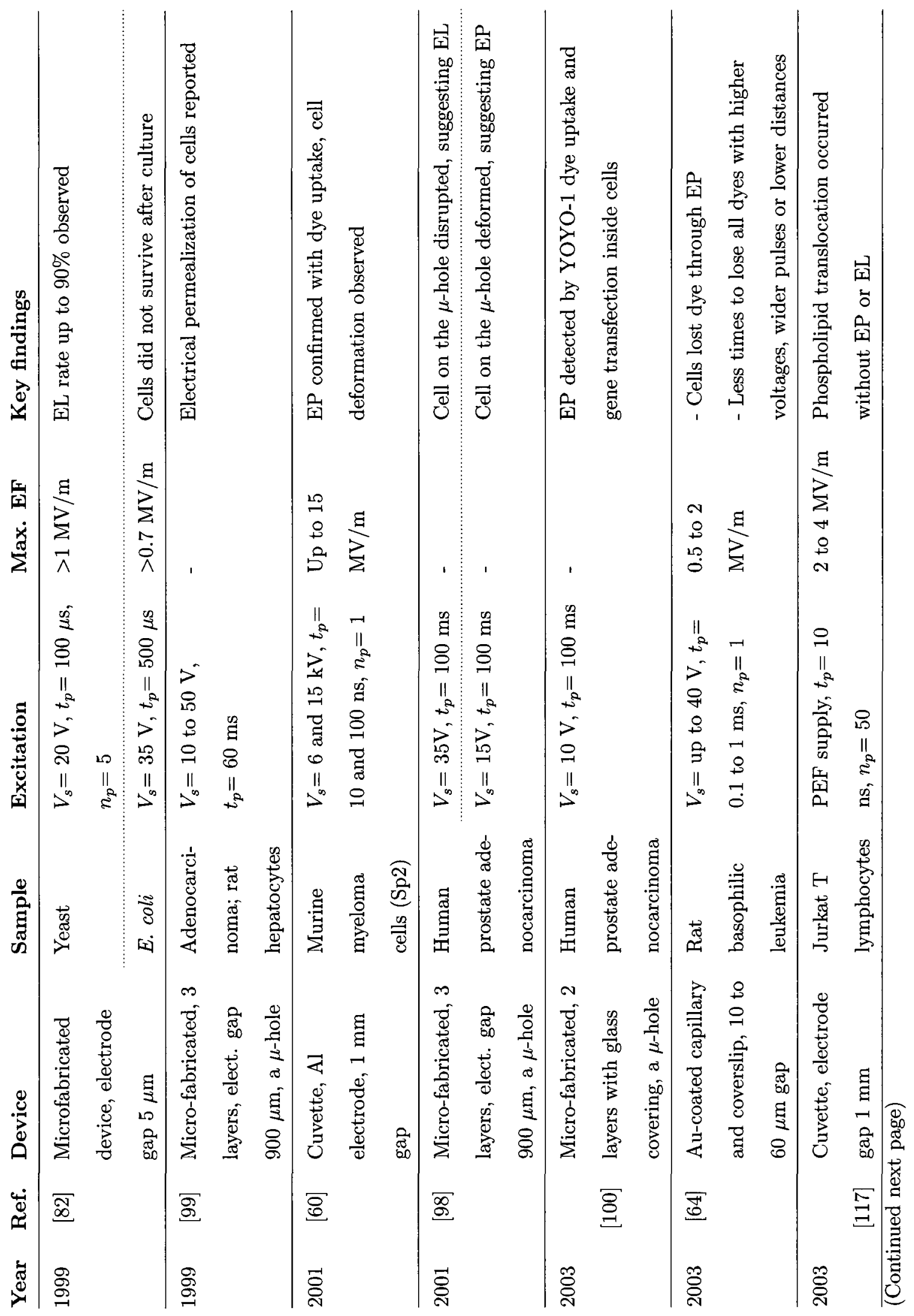




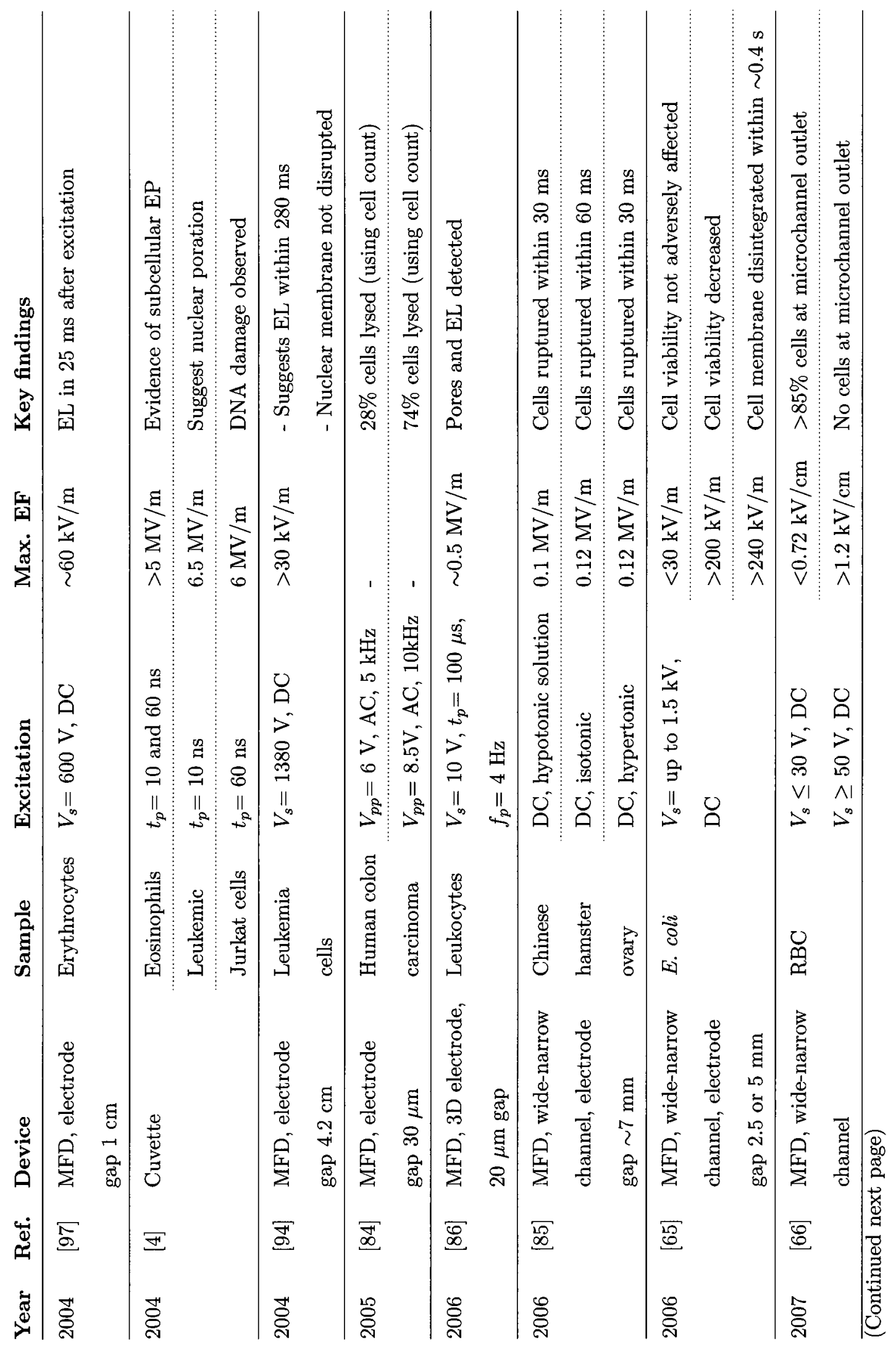




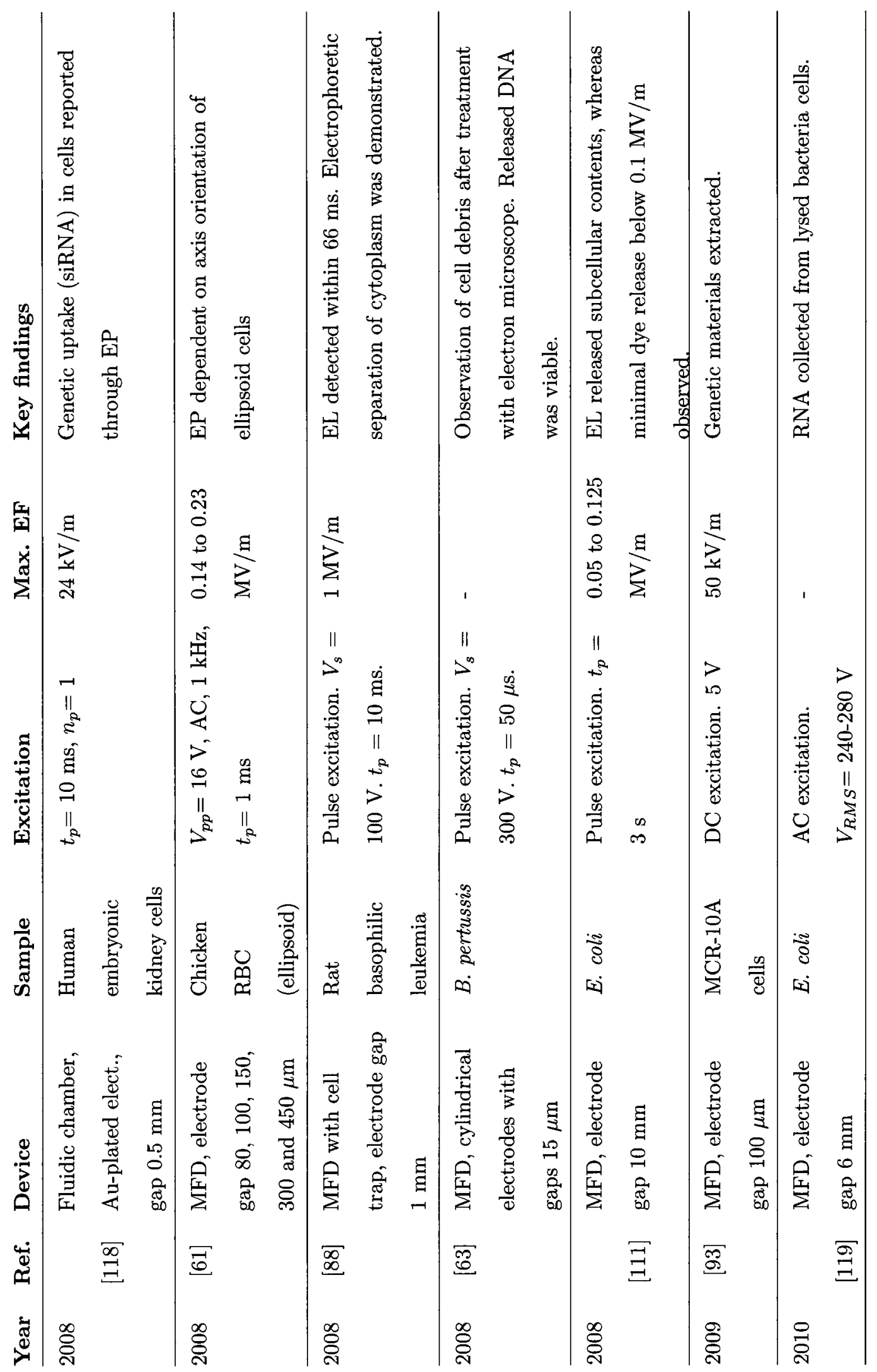


and numerical method $[2,4,101,105,115]$. These modelling approaches are briefly discussed in this section.

\subsubsection{Mathematical model}

This model is derived by solving Laplace equation for the potential caused by an external uniform electric field of strength, $E$, as depicted in Figure 2.2 [1]. In this model, a cell is represented by a sphere with a homogeneous isotropic cytoplasm surrounded by a thin dielectric layer, which represents the cell membrane. The transmembrane potential, $V_{m}$, develops across the cell membrane as $E$ is applied. Considering that the thickness of the cell membrane is very small compared to the cell size, and assuming that the intracellular and the extracellular fluids are purely resistive, $V_{m}$ can be expressed as [1]:

$$
V_{m}=\frac{1.5 E a}{1+a G_{m}\left(\rho_{i}+0.5 \rho_{o}\right)} \frac{1}{1+j \omega T} \cos \delta,
$$

where $a$ is the cell radius, $G_{m}$ is the membrane conductance per unit area, $\rho_{i}$ and $\rho_{o}$ are the resistivities inside and outside of the cell, respectively, $\omega$ represents the angular frequency of the applied potential, $T$ is the characteristic time period, and $\delta$ is the angle between $E$ and the radius vector to $V_{m}$.

As the threshold electric field, $E_{t h}$, is applied and the transmembrane potential becomes higher than the critical value, $V_{c}$, hydrophilic pores begin to form, resulting in EP [112]. When these pore diameters exceed the resealable range of the critical pore diameter (40 $\mathrm{nm}[105]$ ), the pores expand spontaneously causing EL. The minimum time required to achieve critical transmembrane potential is known as the critical time duration, $t_{c}$.

\section{Steady state analysis}

When the pulse duration of the excitation is much larger compared to the relaxation time of the cell membrane, the electric field for a short timescale of the pulse can 


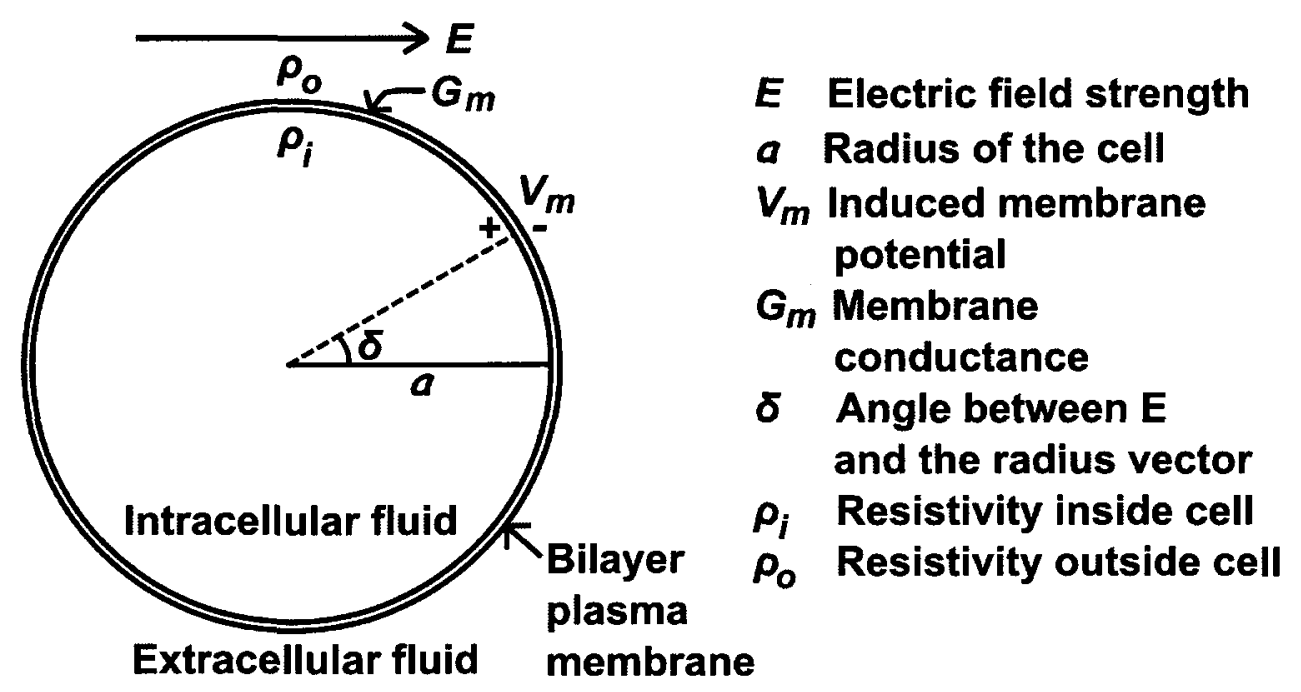

Figure 2.2 Model of a cell exposed to an external electric field showing parameters related to the mathematical model.

be considered to be constant. For the case of a constant electric field (i.e. $\omega=0$ ), expression (2.1) simplifies to [1]:

$$
V_{0}=\frac{1.5 E a}{1+a G_{m}\left(\rho_{i}+0.5 \rho_{o}\right)} \cos \delta,
$$

where $V_{0}$ is the transmembrane potential in the presence of the constant electric field. As membrane resistance is extremely high resulting $G_{m} \approx 0, V_{0}$ becomes $[1,82]$ :

$$
V_{0} \approx 1.5 E a \cos \delta
$$

Note that this expression is only valid when the dielectric relaxation time of the cell membrane is negligible compared to the duration of the applied electric field [82]. Expression (2.3) is the simplest and most widely used model, even recently $[65,66]$.

The critical transmembrane potential, $V_{c}$, occurs at the pole cap regions $(\theta=$ $0^{\circ}, 180^{\circ}$ relative to electric field direction), where $\cos \delta= \pm 1$. The corresponding threshold electric field, $E_{t h}$, is related as,

$$
E_{t h} \approx 2 V_{c} / 3 a
$$

For a generalized shape of cells, a form factor, $f_{f}$, representing the shape characteristic, can be defined, such that Expression (2.4) can be expressed as: $E_{t h}=V_{c} / f_{f} a$. 
For spherical cells, $f_{f}=1.5$, and for cylindrical cells, $f_{f}=l /(l-d / 3)$, where $l$ is

length and $d$ is diameter $[4,109]$. For a constant electric field, an average value of the transmembrane electric field strength, $E_{m}$, can be estimated as: $E_{m}=V_{m} / h$, where $h$ is the thickness of the membrane [102]. The ratio of maximum $E_{m} c$ at polar cap regions to external electric field, $E$, strength can be given as [85],

$$
\frac{E_{m} c}{E}=\frac{f_{f} a}{h} .
$$

Under the condition of low conductivity of the medium surrounding the cell, the previous derivation requires large correction due to surface admittance, membrane conductance, and space charge effect [116]. Neglecting the high frequency relaxation and assuming the surface admittance is real, the transmembrane potential, $V_{0}$, for small cells that includes these correction terms is shown to be [116]:

$$
V_{0}=\frac{1.5 E a \cos \delta}{1+\frac{\rho_{o} G_{s}}{a}+a\left(G_{m}+j \omega C_{m}\right)\left[\rho_{i}+\frac{\rho_{o}}{2}+\frac{\rho_{i} \rho_{o} G_{s}}{a}\right]},
$$

where $G_{s}$ is the surface conductance of the membrane and $C_{m}$ is the membrane capacitance per unit area. If there is no surface conductance, then $G_{s}=0$. For the case of no surface conductance with low frequency of excitation (i.e. $C_{m}$ is negligible), expression (2.6) converges to expression (2.2).

For an alternating electric field, from Expression (2.1), the transmembrane potential, $V_{f}$, for a frequency, $f$, can be written as [1],

$$
V_{f}=\frac{1.5 E a \cos \delta}{\sqrt{1+(\omega T)^{2}}}
$$

where $\omega=2 \pi f$ is the angular frequency and $T$ is the characteristic time period.

\section{Transient response}

The time varying form of the transmembrane potential, $V_{m}(t)$, induced by a step function electric field, $E_{s}$, can be expressed as $[60,86,106]$,

$$
V_{m}(t)=1.5 E_{s} a \cos \delta\left(1-\exp \left(\frac{-t}{\tau_{m}}\right)\right) .
$$


Here $\tau_{m}$ is the charging time constant of the cell membrane, given by, $\tau_{m}=a C_{m}\left(\rho_{i}+\right.$ $0.5 \rho_{o}$ ). For a cell with non-interfering, spherical, membrane-bound organelles, an expression for the internal transmembrane potential, $V_{i m}$, may be expressed as [117],

$$
V_{i m}(t)=1.5 E_{i}(t) a_{i} \cos \theta_{i m}\left(1-\exp \left(\frac{-t}{\tau_{i m}}\right)\right),
$$

where $E_{i}(t)=E_{s} e^{-t / \tau_{m}}$ is the time-varying intracellular electric field, $a_{i}$ is the radius of the organelle, $\theta_{i m}$ is the position angle on the organelle membrane, and $\tau_{i m}$ is the charging time constant of the organelle membrane.

The dissipated electrical energy, $W$, required for membrane breakdown, is $[4,115]$ :

$$
\begin{aligned}
W & =\frac{E_{s}^{2} t_{p}}{\rho_{o}} \\
& =\frac{K t_{p}}{\left[1-\exp \left(-t_{p} / \tau_{m}\right)\right]^{2}}
\end{aligned}
$$

with $K$ being a constant, and $t_{p}$ is the pulse duration of excitation. This expression has a minimum for a pulse duration $t_{p}=1.25 \tau_{m}$. With this pulse duration killing of biological cells by cell membrane disintegration should be the most effective [115].

Once a current, $i$, is induced in a defect (pore), $i^{2} R_{p} t_{p}$ of heat is generated, where $R_{p}$ is the resistance of the membrane pore. This local heating could cause a phase transition or disordering of lipids [101].

\subsubsection{Electric circuit model}

A cell in an electric field can be modelled by the use of electric circuit components, such as resistors and capacitors. Initial scheme was developed by Schwan [120], which was later extended by Schoenbach et al $[105,109,114,115]$. An electric circuit model of a cell enclosed with a BLM cell membrane with one internal organelle (nucleus, which has a double BLM enclosure) is shown in Figure 2.3. Here, the membranes are represented by capacitors $\left(C_{m o}, C_{m i}\right)$ in parallel with resistors $\left(R_{m o}, R_{m i}\right)$, and the intracellular fluids are modelled as only-resistive components $\left(R_{c 1}, R_{c 2}, R_{n}\right)[105,110]$. A voltage source in series with the parallel high resistors are sometimes inserted 


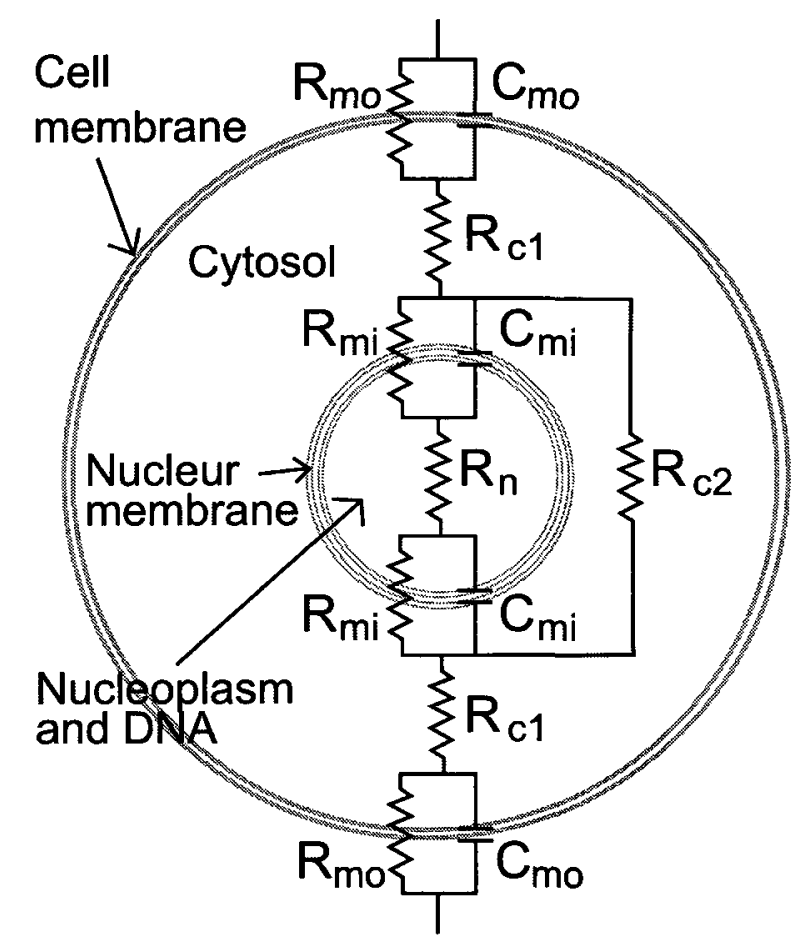

Figure 2.3 Equivalent electrical circuit model of a biological cell (with a nucleus) in a suspension medium exposed to an external electric field.

in the model to represent the voltage-gated channels within the membrane, which controls flow of ions through the membrane depending on the transmembrane potential $[109,114,115]$. However, these voltage sources can be safely ignored when resting transmembrane potential is zero [105]. The capacitive components of the intracellular fluids are neglected due to the fact that pulse durations are long compared to dielectric relaxation times of the fluids [115]. For low frequency applications, the membrane capacitors (i.e. $C_{m o}, C_{m i}$ ) in parallel with the high resistances can be neglected [124]. On the other hand, the parallel resistors (i.e. $R_{m o}, R_{m i}$ ) are neglected for high frequency operations or for short pulse excitations $[4,109,115]$.

An electric circuit model has advantages in the sense that fast circuit simulators can be used to find responses using various types of excitations, and effects on major internal organelle membranes (eg. nucleus) can be easily determined [4]. Simulation results of internal and external membrane potential characteristics for a pulsed external excitation are comparable using analysis results from the mathemat- 
ical model [124].

\subsubsection{Molecular dynamics approach}

Using Molecular Dynamics (MD) approach, cell membranes can be modelled and visualized for a small time scale (mostly ns range) through classical Newtonian mechanics for the dynamical movement of ions and bilayer phospholipid molecules in presence of external forces (eg. an electric field), taking account of many-body interactions within a realistic molecular representation of the biosystem [2]. In this type of model, phospholipids (such as DMPC molecules) are represented by individual atomic potential energy function, with consideration of intermolecular degrees of freedom described by Hooke's law and the bond-stretching and angle-bending, and Fourier series for dihedral angle motion [121].

These DMPC molecules are initially arranged in a bilayer formation inside a bounding box filled with water molecules [122]. Systems are examined in the Normal Temperature and Pressure (NTP) ensemble using 3-dimensional periodic boundary conditions. Interactions and movements of all molecules due to an external electric field excitation are computed for each iteration representing a certain time-step, and used in the next iteration. After steady state is reached through a number of iterations, BLM structure is post-processed and analyzed through suitable analysis and plotting tools. The simulated event time of the system is determined by multiplying the time-step with the number of iterations.

A number of experimental results for EP were verified through MD simulations, such as formation of water channels in the hydrophobic domain of lipid bilayers subject to an electric field [122], movement of dipalmitoyl-phosphatidyle-serine (DPPS) molecule towards anode side of the membrane (PS externalization) [2], suppressed effect of membrane pore formation due to gramicidin/DMPS system [123], stabilization of plasmid in the membrane core after EP [122], the entropy driven nucleation and growth of hydrophilic pores in BLM in the presence of an edge-active agent [104], 
and pore formation times of about 5 to $6 \mathrm{~ns}$ in the cell membrane subjected to a highintensity ultrashort electric pulse [3]. Prime advantage of this modelling approach is that the results closely represent reality and allow us to visualize such events at the molecular level.

\subsubsection{Numerical modelling}

Another approach for biological cell modelling is numerical [105]. This approach allows deriving critical pore radii for initiation of irreversible transition. According to this model, pores formed during electroporation are reversible until a critical diameter, after which the pores continue to expand even if the electric field is withdrawn and the cell death is a consequence [115]. Simulation results using such numerical model shows that the organelle membranes are stressed with shorter pulses (duration of $<1 \mu \mathrm{s}$ ) and the cell membrane is affected by longer pulses because of difference in relaxation times [105].

The stability of a pore depends on the pore energy, which is the sum of mechanical energy, electrical contribution, and other factors [101]. The numerical modelling approach incorporates the Smoluchowski equation that describe the pore distribution function $n(r, t)$ such that the average pore radius $\langle r(t)>$ be [105]:

$$
<r(t)>=\frac{\left[\int_{0}^{\infty} r n(r, t) d r\right]}{\left[\int_{0}^{\infty} n(r, t) d r\right]} .
$$

The pores formed during electroporation process can be characterized by an energy $E(r)$, which is a function of the pore radius $r(t)$ [105]. This $E(r)$ represents the free energy change associated with the formation of a pore and has contributions from three factors: the surface tension of the membrane, line tension of the pore edge, and membrane capacitance. Due to the contribution from the membrane capacitance, $E(r)$ acquires a dependence on the transmembrane potential, $V_{m}(t)$. In the presence of $V_{m}(t)$, this energy function is given by:

$$
E(r, t)=2 \pi h r \sigma(\infty)\left[I_{1}\left(r / r_{0}\right) / I_{0}\left(r / r_{0}\right)\right]-\pi a_{p} V_{m}^{2} r^{2}
$$




$$
E(r, t)=2 \pi \gamma r-\pi \sigma r^{2}+(C / r)^{2}-\pi a_{p} V_{m}^{2} r^{2}
$$

for hydrophobic and hydrophilic pores, respectively. In the above equations $I_{0}$ and $I_{1}$ are the modified Bessel functions of the zeroth and first order, respectively, $h$ is the membrane thickness, $\sigma(\infty)$ is a constant equal to $5 \times 10^{-2} \mathrm{~N} \mathrm{~m}^{-1}$, while $r_{0}(\approx$ $1 \mathrm{~nm}$ ) represents a characteristics length scale over which the properties of water change between the interface and the bulk, $\gamma$ is the energy per unit length of the pore perimeter, $\sigma$ is the energy per unit area of the intact membrane. It is the hydrophilic pores described in expression (2.13), that contribute to EP, and eventual EL $[2,105]$.

The energy function, $E(r)$, is important to the pore dynamics for several reasons [105]. First, it determines the "drift flux" for pores in $r$-space. A positive $E(r)$ slope signifies a driving force toward a smaller radius, while a negative slope forces the growth and expansion of pores in $r$ space. Second, the $E(r)$ profile contains local maxima and minima in $r$ space, which depend on the applied voltage. Finally, $E(r)$ dictates the rate of formation of new pores as a function of $r$. Using the numerical model it is determined that a pore with radius of $20 \mathrm{~nm}$ or more will expand spontaneously in size without any applied voltage as everything in nature prefers to be at the lowest possible energy state $[4,105,115]$. This will lead to irreversible pores contributing to cell lysis. Thus the numerical model enables us to understand pore dynamics.

\subsection{Applications of Electroporation and Electrical Lysis}

Biomedical applications of EP and EL are increasing as we are able to understand these phenomena and to manipulate them. These applications are aided by advancements in fabrication techniques, such as BioMEMS, LOC and Micro Total Analysis System ( $\mu$ TAS). A number of important applications of EP and EL are presented in this section. 


\subsubsection{Electroporation applications}

An important biomedical application of EP is electroinjection or electrotransfection technique that allows introduction of membrane-impermeable molecules into living cells [101]. Examples of such techniques are nucleic acid transport [118], transport of DNA inside Ch. reinh. (green algae cells) [106], Small Interfering Ribonucleic Acid (siRNA) uptake in human embryonic kidney cells [118], and Plasmid DNA (pDNA) transfection into adjacent cells [125]. In addition, EP can be utilized in loading of drugs or ghost materials inside cells, depending on the osmotic balance of the suspending medium [101]. The extent of membrane transfer in these cases can be regulated by adjusting EP parameters [100].

Another application of EP induced death of bacterial cells is to provide $100 \%$ protection against biofouling (undesirable accumulation of microorganism on wetted structures) by applying a high electric field (above $5 \mathrm{kV}$ ) [114]. Ultrashort pulse EP treatment can be used for killing bacteria in water and foods [115], and inducing membrane phospholipid translocation and caspase activation [117]. Some other applications of EP are various types of therapies (cancer, intraocular, vascular), electro-encapsulation, insertion of proteins, activation of membrane transporters and enzymes, and alteration of genetic expression in living cells $[101,112]$. The observed electric field response of $E$. coli in different stages of their growth indicates that rapidly dividing cells are more susceptible to a high electric field than cells which are not proliferating. If the same is true for mammalian cells, then ultrashort pulses of high electric field could reduce tumor or cancer development significantly [109].

\subsubsection{Electrical lysis applications}

Cell lysis using electric field compared to other traditional methods, such as chemical, mechanical, thermal, electro-osmotic, and ultrasound, offers several advantages. Some advantages of this method are fast operation, low power consumption, inexpensive device, microchip compatibility and the potential to be automated. Potential 
applications of EL method are on-chip analysis of cell contents (cytology), genetics, disease diagnosis, oncology, stem cell research, developmental biology, and bacterial decontamination $[63,105,126]$. This method also finds applications in macro-scale, such as energy efficient commercial extractor of crops [107] and water treatment system $[115]$.

\subsection{Other Effects of a High Electric Field on Cells}

In addition to EP and EL, a high electric field might also effect cells in many other ways. These effects are important for various fields of biochemistry and molecular biology. Some of these effects are mentioned below.

\subsubsection{Cellular spin resonance}

Rotation of cells exposed to an external oscillating electric field can be observed for live as well as dead ones. As spinning often starts at a very sharp frequency of the applied field and ceases in the same manner, this phenomenon is called cellular spin resonance (CSR) [1]. This technique may be used to separate cells by their different rotational properties such as characteristic resonance frequency, spinning rate, and spinning sense.

\subsubsection{Electrofusion}

As PEF is applied, adjacent cell membranes might fuse together, a phenomenon known as electrofusion [1]. Initially it was thought to be the result of thermal melting and merging of cell membranes due to Joule heating. However, it is now generally accepted that this phenomenon is due to nonthermal effects related to EP of cell membranes [101]. 


\subsubsection{Electroinsertion}

Purified cloned membrane proteins may be correctly incorporated into a cell membrane using electroinsertion technique. This technique has potential applications in proteomics [112].

\subsubsection{Electroactivation}

An oscillating electric field of appropriate amplitude and frequency can synchronize the conformal oscillation of a protein. If the enforced conformal oscillation is coupled to a ligand binding reaction or a substrate-product conversion, the energy transmitted through the electric field may be captured and transferred to drive an endergonic reaction. Electroconformational coupling has also been used to interpret how a cell or an organism can recognize very weak electric signals [101].

\subsection{Summary}

Electroporation (EP) and electrical lysis (EL) are emerging as effective tools for various applications. Successful transfection of cells with genes and proteins have the potential in single-cell genetic manipulation for effective drug and gene delivery, especially for stem cell research. Successful transfection of cells with dye, genes and proteins have demonstrated the feasibility of single-cell genetic manipulation. Other fields of applications, such as treatment of tumor or cancer cells, advanced genetics and proteomics, decontamination of foods and water, and intraocular and vascular therapy, have demonstrated the potential advantages of using EP over other technologies. On the other hand, EL has many desirable attributes for on-chip cell lysis. However, more research is required to understand and apply this phenomenon effectively.

The exact dynamics of cell EP and EL is still an issue of debate. Some phenomena observed experimentally can not explained with any existing understanding of dynam- 
ics, such as formation of pores at lower inter-electrode voltage in the membrane facing the positive electrode (the hyperpolarized portion of the cell's membrane) than in the membrane facing the negative electrode (the depolarized membrane) [64]. Effects on internal organelles after EP and EL are still unknown.

Modelling of a biological cell under an applied electric field can be performed using various modelling techniques, such as mathematical, electrical, molecular dynamics, and numerical approaches. Mathematical modelling allows to model a wide range of EP related phenomenon. Electric equivalent circuit model has capability to handle various excitation parameters, require small simulation time, and can demonstrate the effect on internal organelles and membranes within the cell. However, the model suffers from lack of ability to model physics after pores are formed. The molecular dynamics approach is effective to visualize events at the molecular level and to understand dynamics involved with EP. Due to high computational complexity, large event time is not feasible to be simulated. In this thesis work, we have extended the current electric circuit model to incorporate dynamics due to pore formation (Chapter 6). 


\section{Chapter 3}

\section{Device Designs}

This chapter discusses the design features of the three devices developed and tested during this research:

1. The Initial Device: This device was fabricated using Protolyne Microfluidic Fabrication technique with no integrated electrodes. This device enabled us to perform initial testing for proof-of-concept of EL of non-invasively collected human fingerprint cells.

2. The Intermediate Device: This device was fabricated using Metal MultiUser MEMS Processes (MetalMUMPs) technology with integrated electrodes. The device testing imposed several challenges, some of which were addressed.

3. The Final Device: This device was fabricated using SensoNit Fabrication Technology that contained many unique features including interdigitated integrated electrodes, cell loading ports above microchannel lysis chamber and ability to perform low voltage EP and EL. The device was successfully tested and met the required objectives. 


\subsection{The Initial Device}

\subsubsection{Background}

Microfluidic devices are able to process micro- or pico -grams of biological elements, enabling miniaturization of laboratory procedures [28]. Thus, our preliminary approach was to develop a Microfluidic Device (MFD) for rapid lysis of sample cells collected from human fingerprints on surfaces by using a high electric field. At the time of this initial study (in 2005), no foundry offered to fabricate microfluidic devices with integrated electrodes. Hence we selected Protolyne Fabrication Technology for our preliminary study from the available list of foundries. This fabrication process was a semi-custom model, where exact locations of the fluidic reservoirs were defined by the foundry. The designer controlled the microchannel parameters, such as length and width, whereas the microchannel height was constrained by the foundry to 20 $\mu \mathrm{m}$.

\subsubsection{Device Design}

Design constraints: The semi-custom fabrication process imposed the following design constraints:

1. The size of the chip was set to $95 \mathrm{~mm} \times 16 \mathrm{~mm}$.

2. There were 8 access holes on a chip. The locations of these access holes were predefined, as shown in Figure 3.1. This constraint limited the minimum microchannel length, which dictated the minimum electrode distance achievable.

3. The etch depth, that defined the height of a microchannel, was constrained to $20 \mu \mathrm{m}$.

Layout of the device: The device was designed using the layout drawing tools L-edit from Tanner Research, Inc. The design consisted of a single layer of microchan- 


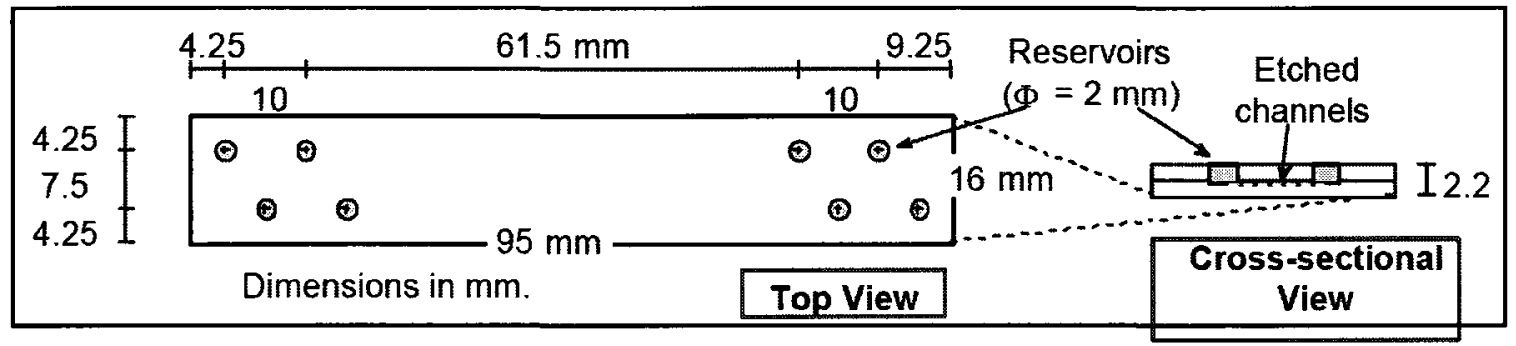

Figure 3.1 Top view and cross-sectional view of the design template showing the predefined fluidic reservoirs locations and the dimension of the device and reservoirs [Adapted from Protolyne manual].

nels, along with the pre-defined fluidic ports and associated etch-pads. The layout of the device is shown in color-plate Figure A.1 to A.3 in Appendix A.

Important design features: Seven microchannels (Figure A.1) of various lengths ranging from $7 \mathrm{~mm}$ to $55 \mathrm{~mm}$ were designed that connected various reservoirs. The left hand side of the device contained two intersecting microchannels of $7 \mathrm{~mm}$ and $14.5 \mathrm{~mm}$, as illustrated in Figure A.2. All channels in this section had a drawn width of $60 \mu \mathrm{m}$, resulting in a microchannel width of $100 \mu \mathrm{m}$, as the fabrication process results $20 \mu \mathrm{m}$ of additional etching on each side of the microchannel to the drawn width. This is due to the chemical etching process of the microchannels that etches isotropically in all directions. Figure A.3 shows the right hand section of the device containing 4 more microchannels of $7 \mathrm{~mm}, 8 \mathrm{~mm}$ and $14.5 \mathrm{~mm}$ long. All channels of this section have a drawn width of $40 \mu \mathrm{m}$, resulting in $80 \mu \mathrm{m}$-wide microchannels. The microchannel widths were chosen such that it allowed less interference of cell flow through the microchannel, as well as reduced the power consumption. Hence, the microchannel widths were set to about 3 to 4 times larger than the average diameter of cells (about $25 \mu \mathrm{m}$ ). Note that all microchannels terminated at the reservoir ports, not at the etch-pads, as the top of the trenches became exposed inside the reservoir ports (Figure 4.9). In other words, the length of a microchannel was defined by its intersection with a fluidic reservoirs, not with an etch-pad. 


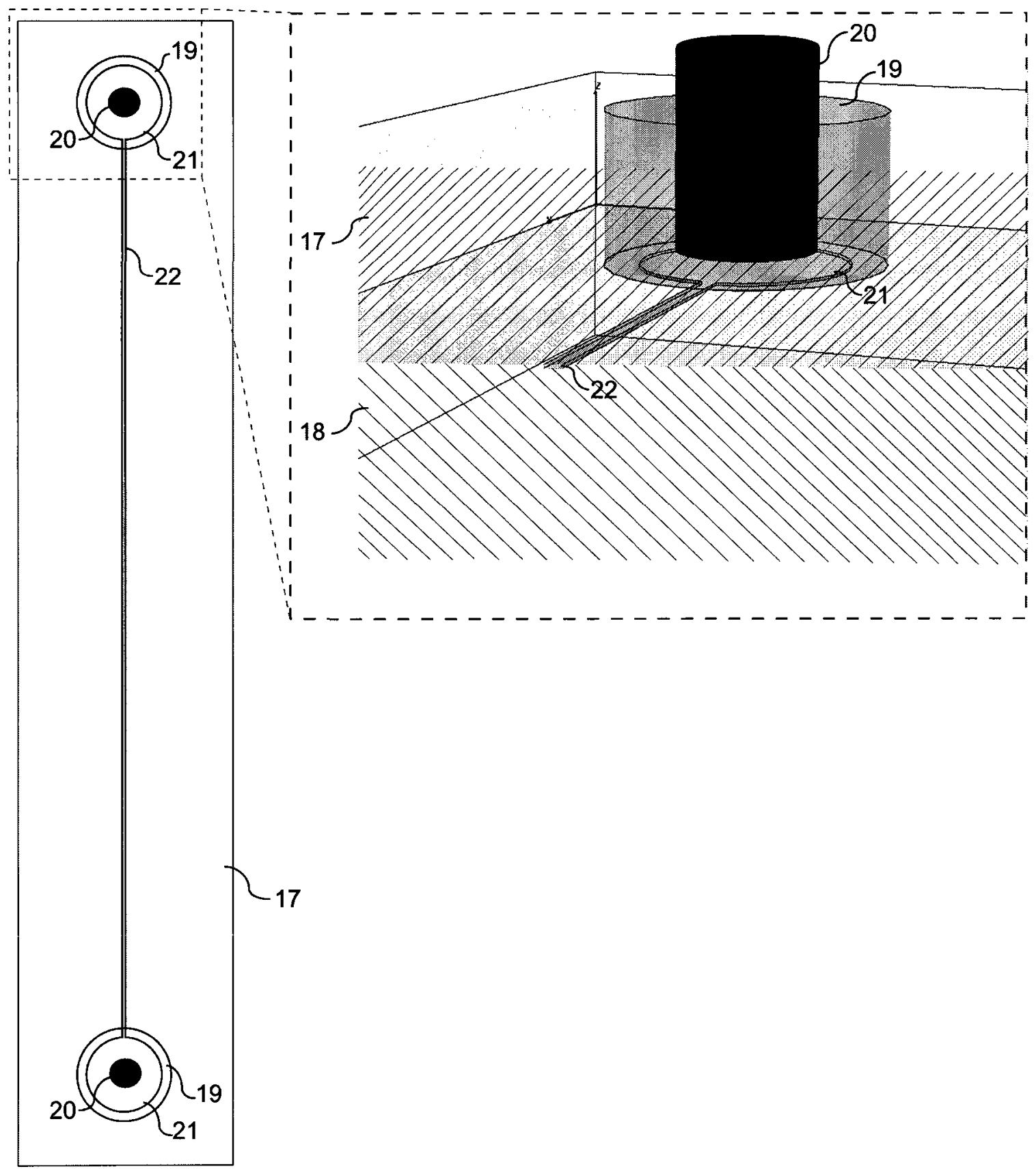

Figure 3.2 A sketch and a 3D model showing a microchannel structure design in details. Legends: 17- Top glass slide, 18- Bottom glass slide, 19Reservoir, 20- External electrode, 21- Etch pad, 22- Microchannel. 


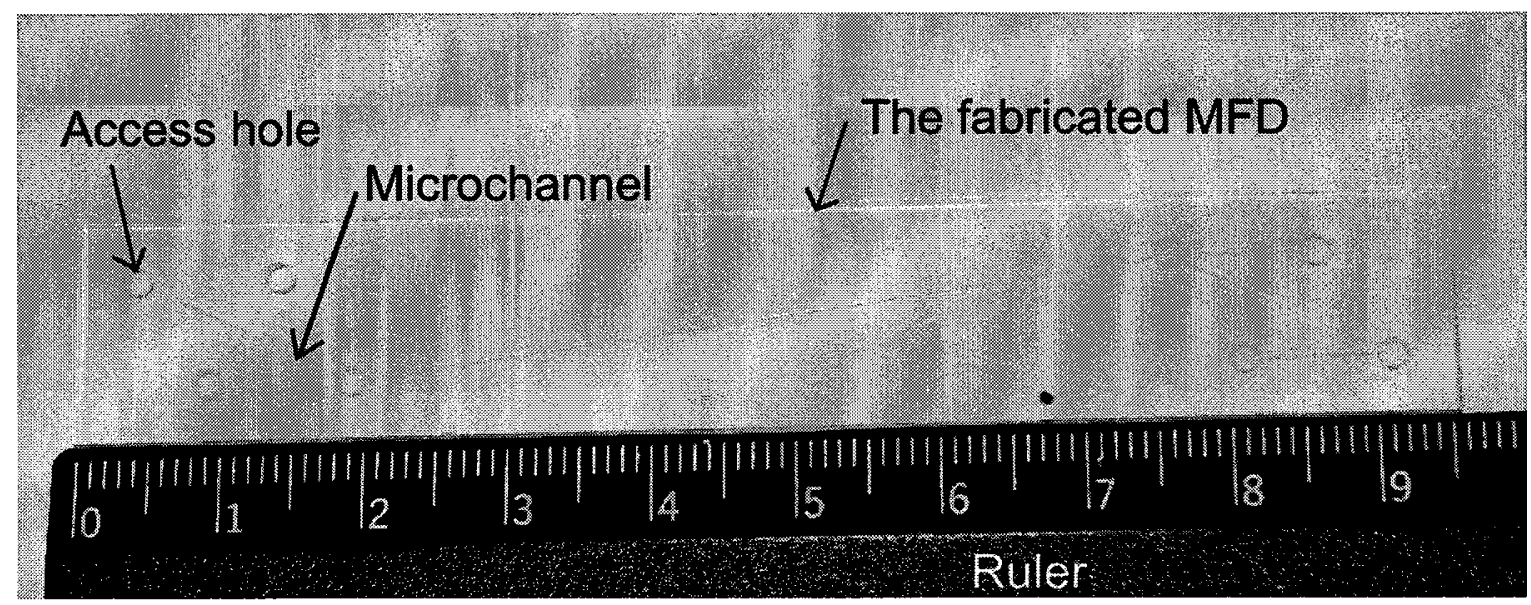

Figure 3.3 A photograph of the fabricated microfluidic device with a cmruler beside it.

\subsubsection{Fabrication}

The device was fabricated using Protolyne Fabrication Technology from Micralyne Inc. of Edmonton, Alberta, Canada. The fabrication cost was partially supported ( $80 \%$ of total cost) by a grant from Canadian Microelectronics Corporation (CMC). The fabricated device consisted of two fused glass-slides (Schott Borofloat), with dimensions of $95 \mathrm{~mm} \times 16 \mathrm{~mm} \times 1.1 \mathrm{~mm}$ each. Eight predefined fluidic ports, that acted as reservoirs, of $2 \mathrm{~mm}$ diameter were through-drilled in the top slide (Figure 3.1). Eight etch-pads of $1.5 \mathrm{~mm}$ in diameter, aligned with the reservoirs (access holes), and several trenches or grooves according to the user-layout were etched using isotropic chemical bulk-etching process on the top surface of the bottom slide. These trenches formed the microchannels when the two glass slides were fused together.

A photograph of the fabricated microfluidic device, along with a ruler, is shown in Figure 3.3. The photograph shows the predefined access holes (i.e. the reservoirs) and the etched microchannels. Scanning Electron Microscope (SEM) photographs of the trenches on the top surface of the bottom slide are shown in Figure 3.4 and Figure 3.5. From these SEM images, it was noted that the etching process leaves some irregularities on the surface of the trench, especially on the channel walls. 


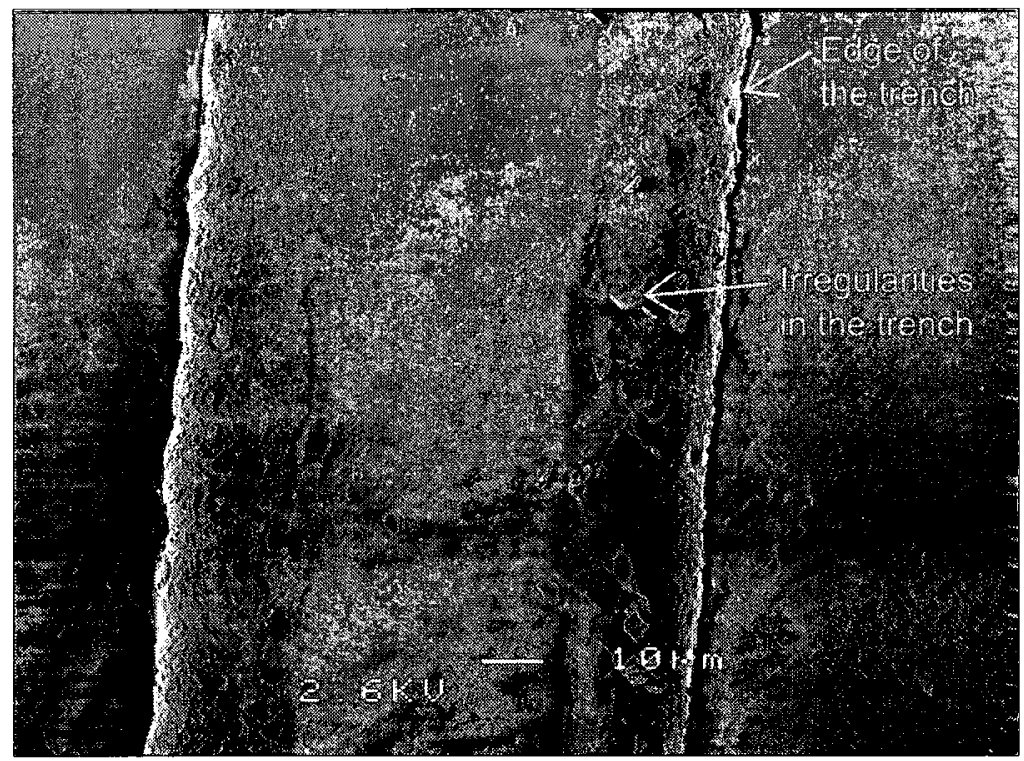

Fignnre 3.4 A SEM photograph of a microchannel of the fabricated microfluidic device showing surface roughness of an etched trench (microchannel) on the top surface of the bottom slide after removing the top slide.

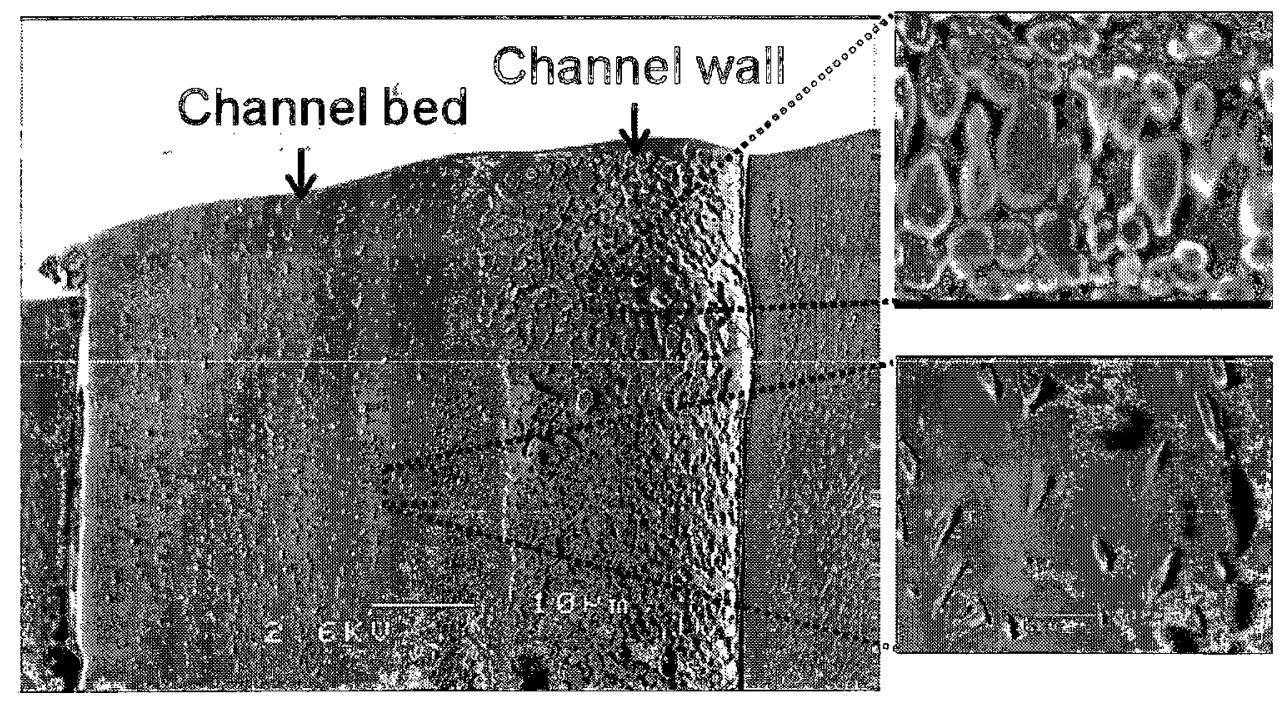

Figrure 3.5 SEM photographs taken of an microfuidic device that was sliced off perpendicular to a microchannel length and the top glass-slide removed. The bottom slide was vertically placed with a $45^{\circ}$ horizontal-tilt. The photograph shows the surface roughness on the channel bed and the channel wall. These surfaces were imaged at a higher magnification and shown to the right. 


\subsection{The Intermediate Device}

\subsubsection{Background}

After experiments with the initial device, the concept of cell lysis with a high electric field was demonstrated. But the required voltage was very high (about $800 \mathrm{~V}$ ), unacceptable for medical devices in contact with human body and unsuitable for implementing a portable device. In an effort to reduce this voltage requirement, integrated electrodes that can be fabricated in micron distance was sought. At the time of this research phase (in 2006), no microfluidic device foundry offered integrated electrode. Hence we looked for alternatives and Microelectromechanical System (MEMS) fabrication process, in particular MetalMUMPs, was selected to test the concept of voltage reduction using integrated electrodes for Electrical Lysis (EL) to enable a low supply voltage operation.

\subsubsection{Design and fabrication of the intermediate device}

MetalMUMPs fabrication technology consisted of several layers that included a thick metal layer. We used this metal layer to fabricate integrated electrodes to lower the voltage requirements for EL. A list of layers with brief descriptions is presented in Table 3.1.

\begin{tabular}{|c|c|c|c|c|}
\hline $\begin{array}{l}\text { Materia } \\
\text { Layer }\end{array}$ & $\begin{array}{l}\text { Thlokness } \\
\text { (pm) }\end{array}$ & $\begin{array}{l}\text { Wthography Level } \\
\text { Name }\end{array}$ & S. Lithography Level Pupose & Comments \\
\hline Isolation Oxide & 2.0 & & & $2.0, \mathrm{~m}$ thermal oxide \\
\hline Oxide 1 & 0.5 & OXIDE1 & Define trench; release Nitride & $0.5 \mathrm{fm}$ PSG \\
\hline Nitride 1 & 0.35 & & & $\begin{array}{l}0.35 \mu \mathrm{m} \text { low-stress } \\
\text { silicon nitride }\end{array}$ \\
\hline Poly & 0.7 & POLY (HOLP) & Pattern Poly & $\begin{array}{l}0.7 \mu \mathrm{m} \text { doped } \\
\text { polysilicon }\end{array}$ \\
\hline Nitride 2 & 0.35 & NITRHOLE & $\begin{array}{l}\text { Define areas where Nitride is removed. Define trench. } \\
\text { Open hole for electrical contact between Metal and Poly }\end{array}$ & $\begin{array}{l}\text { Patterns both Nitride } 2 \\
\text { and Nitride } 1\end{array}$ \\
\hline Oxide 2 & 1.1 & METANCH & \multirow{2}{*}{$\begin{array}{l}\text { Open holes to anchor Metal to Nitride or Poly. Open holes } \\
\text { for thin melal traces of Anchor Metal }\end{array}$} & $1.1 \mu \mathrm{mPSG}$ \\
\hline Anchor Metal & 0.035 & METANCH & & $10 \mathrm{~nm} \mathrm{Cr}+25 \mathrm{~nm} \mathrm{Pl}$ \\
\hline Plating Base & 0.55 & & & $500 \mathrm{~nm} \mathrm{Cu}+50 \mathrm{~nm} \mathrm{Ti}$ \\
\hline Metal & 20.5 & METAL (HOLM) & Pattern Metal & $20 u \mathrm{~m} \mathrm{Ni}+0.5 \mu \mathrm{m} \mathrm{Au}$ \\
\hline Sidewall Metal & $1.0-3.0$ & GOLDOVP & Define area for Sidewall Metal and pattem Sidewall Metal & $1-3 \mathrm{um} \mathrm{Au}$ \\
\hline
\end{tabular}

Table 3.1 A table showing various mask layers of the MetalMUMPs fabrication process and brief descriptions of layers. [Adapted from MetalMUMPs Design Handbook] 


\subsubsection{Rationale}

The following rationales lead us to fabricate and test a device based on this technology.

1. Integrated electrodes at gaps of $20 \mu \mathrm{m}$ are about 500 times smaller compared to the initial device. Using analytical expression for electric field, about 500 fold (neglecting the estimation error) voltage reduction was expected.

2. MetalMUMPs fabrication process results trench and surfaces with less irregularity compared to the initial device. This would reduce cell adherence tendency due to smoothness of surface as well as reduced Zeta Potential resulting from Si substrate.

3. MetalMUMPs technology shielded metal layers with a gold plating, that will reduce metal electrodes degradation during the experiments with electric current conduction through the fluids.

4. MetalMUMPs technology allowed $25 \mu$ m deep trenches in Si substrate, that can be potentially utilized to make microchannels or reservoirs.

5. MetalMUMPs technology allowed a polysilicon layer that could potentially serve as heater or temperature sensor for integration of later steps of DNA processing.

\subsubsection{Important design features}

The device design oriented around four reservers in four corners of the chip. Four sets of channels and four electrode orientation topologies were designed in between adjacent reservoirs. The layout of the complete chip is shown in Figure A.4, which was designed using MEMSPro tool from SoftMEMS corp., USA. A brief description of different electrode sets is given here.

1. In Electrode Set 1 (Figure A.5), electrode-pairs were positioned across the channel. There were 20 electrode-pairs in 5 groups, each group having different gaps and widths. Electrode gap was between $20 \mu \mathrm{m}$ to $50 \mu \mathrm{m}$. 
2. Electrode Set 2 contained 3 channels and 3 electrode-pairs (Figure A.6). Channels were narrow at the center and wider at both ends with curved sidewalls. Electrode-pairs were positioned across the narrow section of each channel. Gap between electrode-pairs were $20 \mu \mathrm{m}$ and width varied between $20 \mu \mathrm{m}$ to 100 $\mu \mathrm{m}$.

3. Electrode Set 3 had electrodes along the channel walls (Figure A.7). Gap between electrodes (as well as channel width) reduced towards middle. 20 electrodes were designed with various gaps in 5 different groups. Electrode gaps were between $20 \mu \mathrm{m}$ to $90 \mu \mathrm{m}$.

4. Electrode Set $4 \mathrm{a}$ and $4 \mathrm{~b}$ had a composition of 20 electrode-pairs as shown in Figure A.8. Each electrode-pair had two different gap settings between $40 \mu \mathrm{m}$ to $140 \mu \mathrm{m}$.

\subsubsection{Fabrication}

The fabrication process flow of MetalMUMPs technology is described briefly below.

1. Base wafer: An N-type $<100>$ silicon ( $\mathrm{Si}$ ) wafer is used as the substrate.

2. Isolation Oxide: A $2 \mu \mathrm{m}$ layer of silicon oxide $\left(\mathrm{SiO}_{2}\right)$ is grown on the wafer surface to provide electrical isolation from the substrate.

3. Oxide 1: A $0.5 \mu \mathrm{m}$ thick polysilicate glass (PSG) sacrificial layer is grown and patterned using OXIDE1 mask.

4. Nitride 1: A blanket layer of $0.35 \mu \mathrm{m}$ low-stress silicon nitride $\left(\mathrm{Si}_{3} \mathrm{~N}_{4}\right)$ is deposited, followed immediately by the deposition of $0.7 \mu \mathrm{m}$ polysilicon layer and patterning with POLY mask. $\mathrm{Si}_{3} \mathrm{~N}_{4}$ layer provides a protective encapsulation of polysilicon during bulk etching of Si.

5. Nitride 2: This is a second layer of $0.35 \mu \mathrm{m}$ thick low-stress $\mathrm{Si}_{3} \mathrm{~N}_{4}$, then all nitride layers are patterned with the third mask level, NITRHOLE. 
6. Oxide 2: Another sacrificial oxide layer of $1.1 \mu \mathrm{m}$ is grown and pattered with the mask, METANCH.

7. Anchor Metal: A liftoff process is used to provide thin layers of $\mathrm{Cr}$ and $\mathrm{Pt}$ (Anchor Metal) only in the bottom of the Oxide 2 anchors.

8. Plating Base: A blanket metal layer of $500 \mathrm{~nm}$ of $\mathrm{Cu}$ protected with a thin Ti layer acts as the Plating Base layer that provides electrical continuity.

9. Metal: The fifth mask level, METAL, is patterned using a thick resist to form the stencil for the electroplated metal layer and a $20 \mu \mathrm{m}$ of nickel is electroplated into the patterned resist. Subsequently a $0.5 \mu \mathrm{m}$ gold layer is plated on top of the nickel to provide a suitable pad material for wire bonding of external electrical connections.

10. Sidewall Metal: This final plated metal layer is a 1-3 $\mu \mathrm{m}$ gold layer that provides a highly reliable low resistance electrical contact. This step uses the GOLDOVP mask layer.

11. Release of Si: This final stage form is to etch a $25 \mu \mathrm{m}$ deep trench in the $\mathrm{Si}$ substrate in the areas defined by the OXIDE1 and NITRHOLE masks.

The fabricated chip or die was packaged in a 68-Pin Grid Array (PGA) package. The package selection was based on two factors. Firstly, the chip surface needed to be exposed to allow introduction of fluids and cells during the experiments. Secondly, a large number of pins were needed to allow all internal electrodes to be accessible from an external excitation source. A photograph of the packaged device is shown in Figure 3.6. The device was photographed with a SEM microscope to observe surface irregularities (Figure 3.7). The irregularities were significantly lower compared to the initial device. 


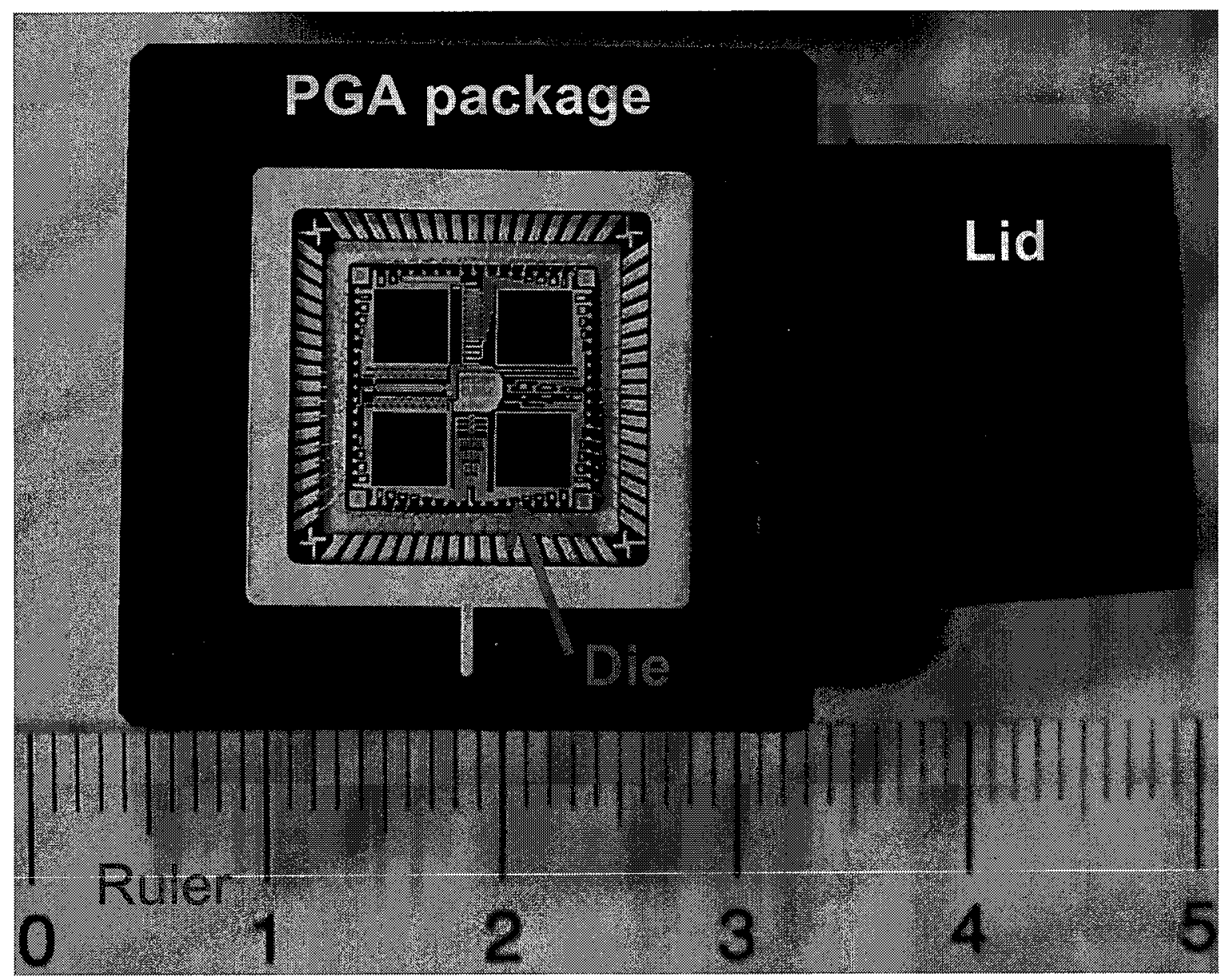

Figure 3.6 A photograph of the fabricated and packaged chip (lid uncovered) with a $\mathrm{mm}$ ruler beside it. All pins are at the bottom of the package. 


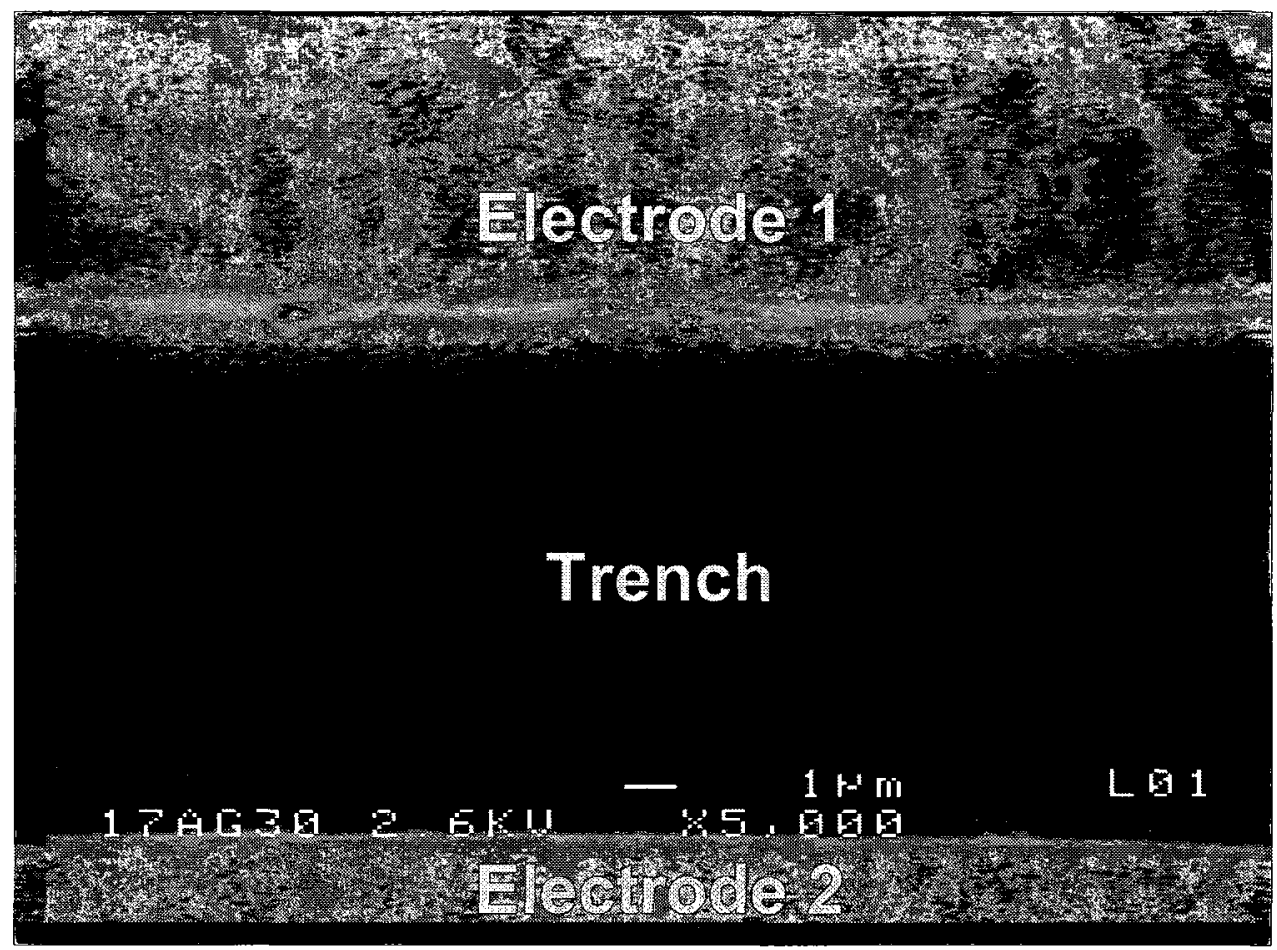

Figure 3.7 A SEM photograph of two electrodes of Electrode Set 3 showing little surface roughness. A scale bar is indicated.

3.3 The Finall Device

\subsubsection{Background}

After experimentation with the intermediate (second) device, several challenges were identified for microfluidic application in MEMS based device, in particular the fluidic encapsulation, optical transparency, chemical and biological compatibility etc. In the meantime, a microfluidic foundry offered integrated electrode fabrication facility in late 2007. We have designed our third and final device incorporating our acquired knowledge with the previous two devices, as well as several novel attributes.

\subsubsection{Desigm of the final device}

A third and final Biomedical Microelectromechanical System (BioMEMS) device for this study was designed and fabricated using Sensonit technology. The first com- 
mercial fabrication batch through CMC was in late 2007. There were two different designs submitted early 2008 in the second batch offered by CMC. The devices were designed using MEMSPro tool (SoftMEMS corp., USA). The fabricated devices were delivered in mid 2008. We are testing one of them at the Cardiovascular Device Division of Ottawa Heart Institute. Findings from this device is briefly discussed in this section. Detailed discussion will be provided later in the relevant thesis work.

\subsubsection{Rationale}

The Sensonit technology was developed for microfluidic applications requiring integrated electrodes inside microchannels. Hence, the fabrication was the most suitable technique for us to pursue. Rationales that motivated us to use this fabrication technique are outlined below.

1. The technology allows integrated electrodes as close as $10 \mu \mathrm{m}$ apart.

2. The technology is full-custom, allowing flexible design choices.

3. The fabricated device is optically transparent (except where electrodes are placed) allowing observations under optical microscopes.

4. The fabrication allows fluidic ports that can be used for proper fluidic handling.

\subsubsection{Important design features}

The device design consisted many elements of Deoxyribonucleic Acid (DNA) detection process, like cell lysis, Polymerase Chain Reaction (PCR), capillary electrophoresis separation of DNA. However, only the cell lysis element is discussed here, which is relevant to this thesis.

The complete layout of the chip is shown in Figure A.9. The complete device was $3 \mathrm{~cm}$ long, $1.5 \mathrm{~cm}$ wide and $1.8 \mathrm{~mm}$ thick. The lower half of the chip was designed for EL, as well as Electroporation (EP). This section is indicated as the cell lysis 
chamber in the figure. An enlarged version of the section $\mathbf{A}$ of this cell lysis chamber is shown in Figure A.10. There are three mask layers: (integrated) electrode mask, microchannel mask and port mask. There were three types of ports designed in this device: fluidic ports, electrode ports and cell ports.

The cell lysis chamber composed of 5 parallel microchannels of $400 \mu \mathrm{m}$ width, having integrated electrodes placed in interdigitated configuration inside the microchannels. The interdigitated electrodes, noted as fingers, were $10 \mu \mathrm{m}$ wide and $10 \mu \mathrm{m}$ spaced from each other. This configuration of fingers achieves following advantages:

1. It minimizes the applied voltage requirement. The required voltage is limited by the minimum electrode gap imposed by the fabrication technology.

2. This configuration maximizes the effective area ratio of high electric fields to at least $50 \%$ coverage.

3. The effective area of high electric fields can be large enough to contain a large number of cells.

4. The fingers were underneath the microchannel surface allowing free flow of fluids in any horizontal direction.

The fingers were alternatively connected to positive and negative electrode ports via large sections of electrodes with width $\geq 0.4 \mathrm{~mm}$, providing low resistance paths from interdigitated electrodes (fingers) to electrode ports. These large electrodes are noted as thick electrodes.

Another important feature of the device was that the cell ports were placed directly above the microchannels, providing easy access paths for sample cells into these microchannels. Cell ports also facilitate the washing step, reducing channel clogging. These two features, interdigitated electrodes and placement of cell port on top of microchannels with electrodes, are novel design featured of such device, a significant contribution of this study. 


\subsubsection{Fabrication}

The fabrication process of Sensonit devices consists of the following fabrication steps:

1. Define microchannels: The bottom surface of the $1.1 \mathrm{~mm}$ thick top glass slide is etched $40 \mu \mathrm{m}$ deep to form trenches using channel mask.

2. Define ports: The ports are crated using port mask on the top slide by powder blasting process, resulting an upside down truncated conical cylinder shaped hole through the top slide, so that the top side diameter is the same as the drawn diameter and the bottom side diameter is $0.8 \mathrm{~mm}$ smaller than the top side opening.

3. In-channel electrodes: Electrode mask is used to define etch-cavities of $200 \mathrm{~nm}$ deep on the top of the bottom glass slide. Then a $20 \mathrm{~nm}$ tantalum (Ta) and 180 $\mathrm{nm}$ platinum $(\mathrm{Pt})$ are deposited and pattered inside these cavities, and serve as integrated electrodes.

4. Binding: The final fabrication step involves direct bonding of the top and bottom glass slides to form the complete assembly.

A photograph of a portion of a cell lysis chamber of the fabricated device as observed under a reflected light confocal optical microscope is shown in Figure 3.8. Here a cell port can be seen on top of a microchannel. Integrated electrodes are seen in a lighter color in this figure. Fingers in interdigitated configuration are located underneath the microchannel (and the cell port). The electrodes inside the cell port are not in focus, due to different reflective indexes of glass and air (inside the cell port).

\subsection{Discussion on the Novelty of the Final Device}

A sketch of the top view and cross sectional view of the cell lysis chamber of the final device is shown in Figure 3.9 and Figure 3.10, respectively. 


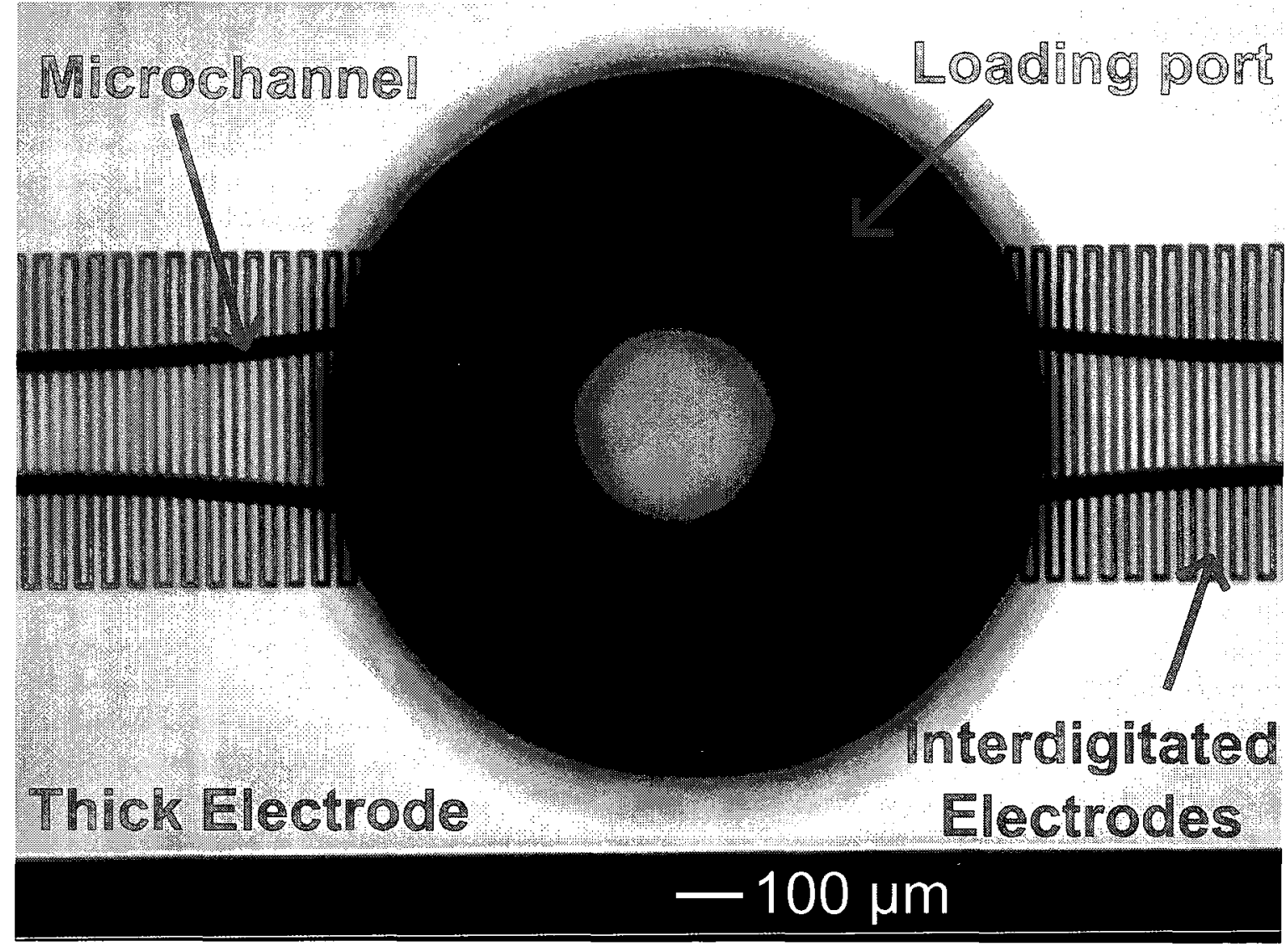

Figure 3.8 A photograph of a portion of the cell lysis chamber on the chip, showing a cell port, a section of a microchannel, and integrated electrodes (interdigitated and thick). The photograph was taken by a reflected light confocal optical microscope. Note that the metal layer is seen in a lighter color due to light reflection. 


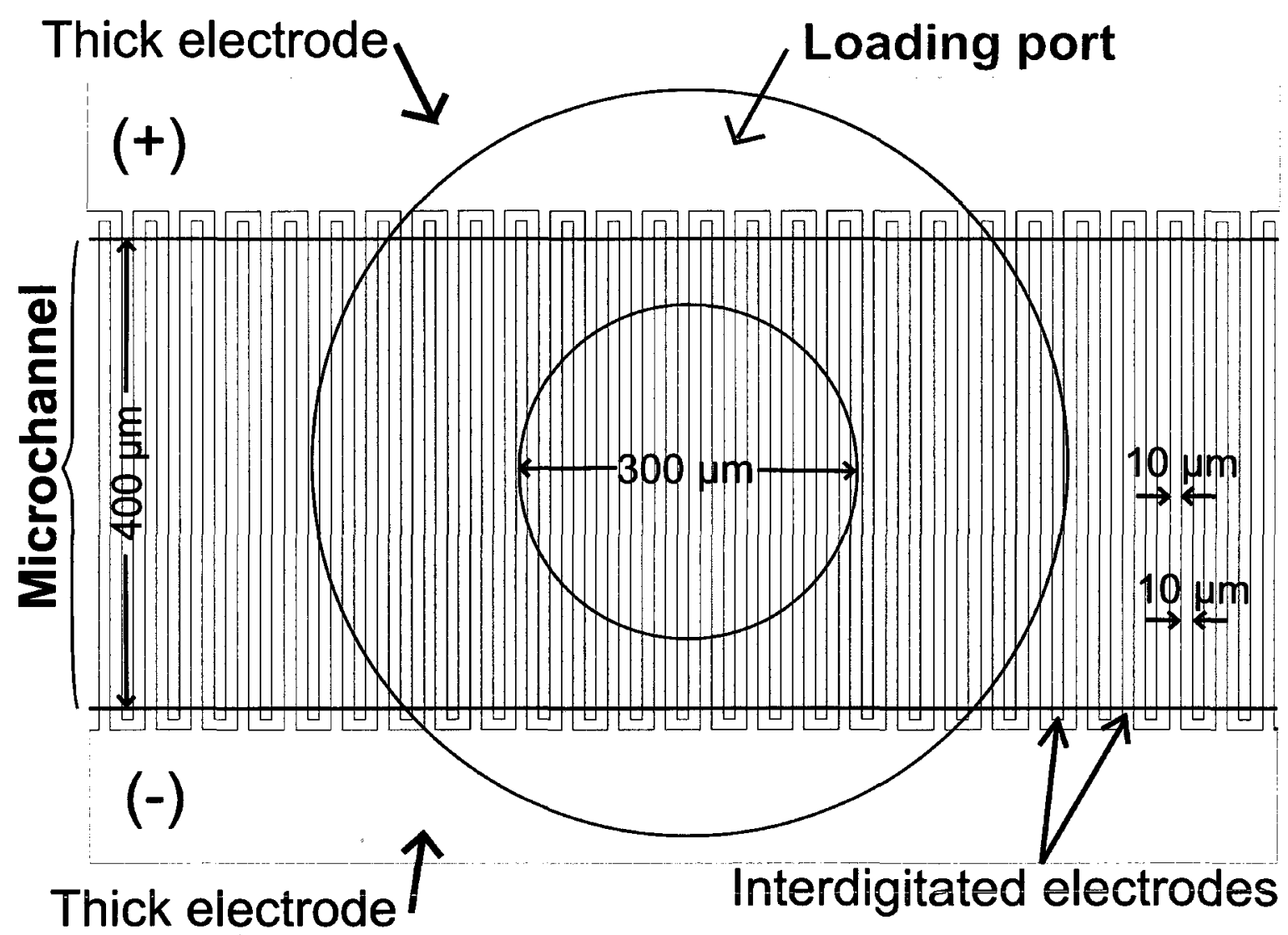

Figure 3.9 A sketch showing the top view of the final device. The configuration of two types of integrated electrodes are shown: i) Interdigitated electrodes (fingers), ii) Thick electrodes. Relevant dimensions of the tapered loading ports, electrodes and microchannel are indicated. 


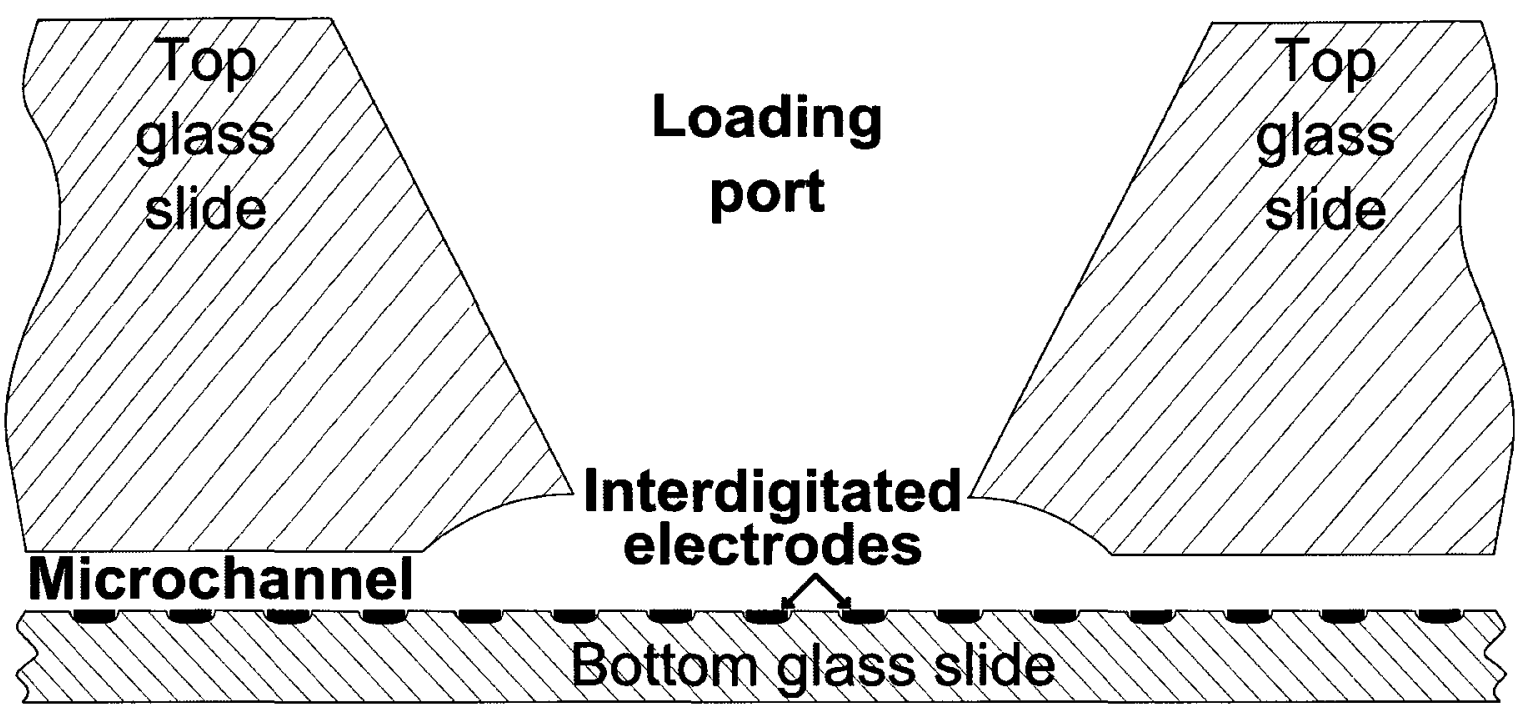

Figure 3.10 A sketch showing the cross section of the final device along a loading port demonstrating configuration of integrated electrodes and microchannels.

Novel attributes achieved with our final device are listed here.

1) Cell size: The electrodes in the final device are on the bottom of the microchannel. Thus microchannel width $(400 \mu \mathrm{m})$ or height $(40 \mu \mathrm{m})$ is not restricted to the gap between electrodes $(10 \mu \mathrm{m})$ (Refer to Figure 3.9 for details). This electrode configuration creates higher electric field at the bottom of the microchannel, where the cells settle. This difference in electrode configuration compared to other similar reported devices in literature allowed us to have electrode gaps much less compared to the diameter of the cells, which is not possible in the other reported devices. For instance, the cell diameter of our sample was 20 to $30 \mu \mathrm{m}$, larger than electrode gap.

2) Optimal use of electric field: The electrodes in the final device are configured in inter-digitized configuration without pointed edges (Figure 3.9). The configuration facilitates a uniform electric field between the electrode gaps. The electrode gap is restricted by the fabrication limit. The uniform electric field occupies $50 \%$ of the lysis area within the microchannel and enables cells to be lysed without aligning them along the pointed edges as required in the other devices being compared.

3) Cell positioning time: In the final device, a high electric field is developed 
simultaneously among all electrode gaps within the cell lysis chamber, so that any part of a cell within the gap will experience the high electric field and will be lysed. Hence there is no delay required for cell positing compared to other reported devices, and reduced time and complexity is achieved.

4) Fast sample introduction ability: The samples in the final device are introduced through the loading ports directly above the microchannels. This leads to a shorter sample load time compared to other similar devices reported.

5) Reusability: The device configuration of sampling (loading) port above the wide microchannels allowed reliable cleaning of the microchannels without microchannels being clogged by cell debris, as the washing fluid can enter and exit through sampling ports with ease.

6) Power supply requirement: The final device operating procedure requires only one low voltage power supply of pulsed type. This results in a lower cost, simplicity, portability, and requires simpler circuitry, as well as a simpler operation protocol.

\subsection{Summary}

This chapter discusses on the devices developed in this research. The initial device was used to verify the concept and have the first experience of the researchers to the issues involved. The intermediate device enabled transition to the final device, which includes a number of attributes that resulted optimal performance of EP and EL. 


\section{Chapter 4}

\section{Simulation Results}

This chapter discusses the modelling and simulations of the devices for electric field and temperature.

\subsection{Simulation of Electric Field Inside Microchan- nels}

A number of Microfluidic Devices (MFDs) reported in the literature share the same topology as the MFD that was fabricated in this study $[47,65,66,97]$. Hence, an analysis of this MFD might be useful for other researchers on relevant area. Keeping this in mind, our analysis extends beyond the fabricated device dimensions to extract generalized trends.

Before proceeding to a comprehensive analysis, a qualitative comparison is made for a case without any microchannel (Figure 4.1) and a case with microchannel structures (Figure 4.2). Finite Element Method (FEM) simulations of two cases show that microchannel structures confine electric fields within the microchannels. Thus in the latter case, less energy is spent to produce a certain electric field and the effective area of operation extends along the microchannels where cells would be positioned. 


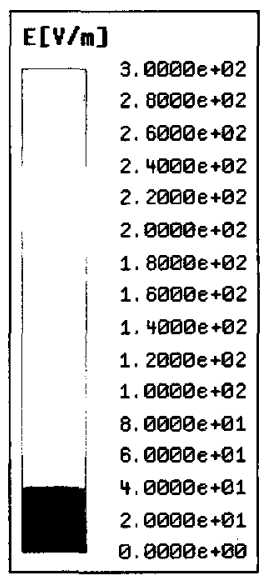

\section{$\underline{1 \mathrm{~mm}}$}

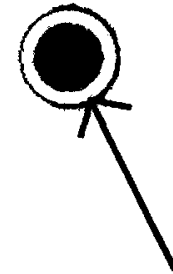

3

\section{Electrode}

Figure 4.1 FEM simulation of an MFD without a microchannel structure.

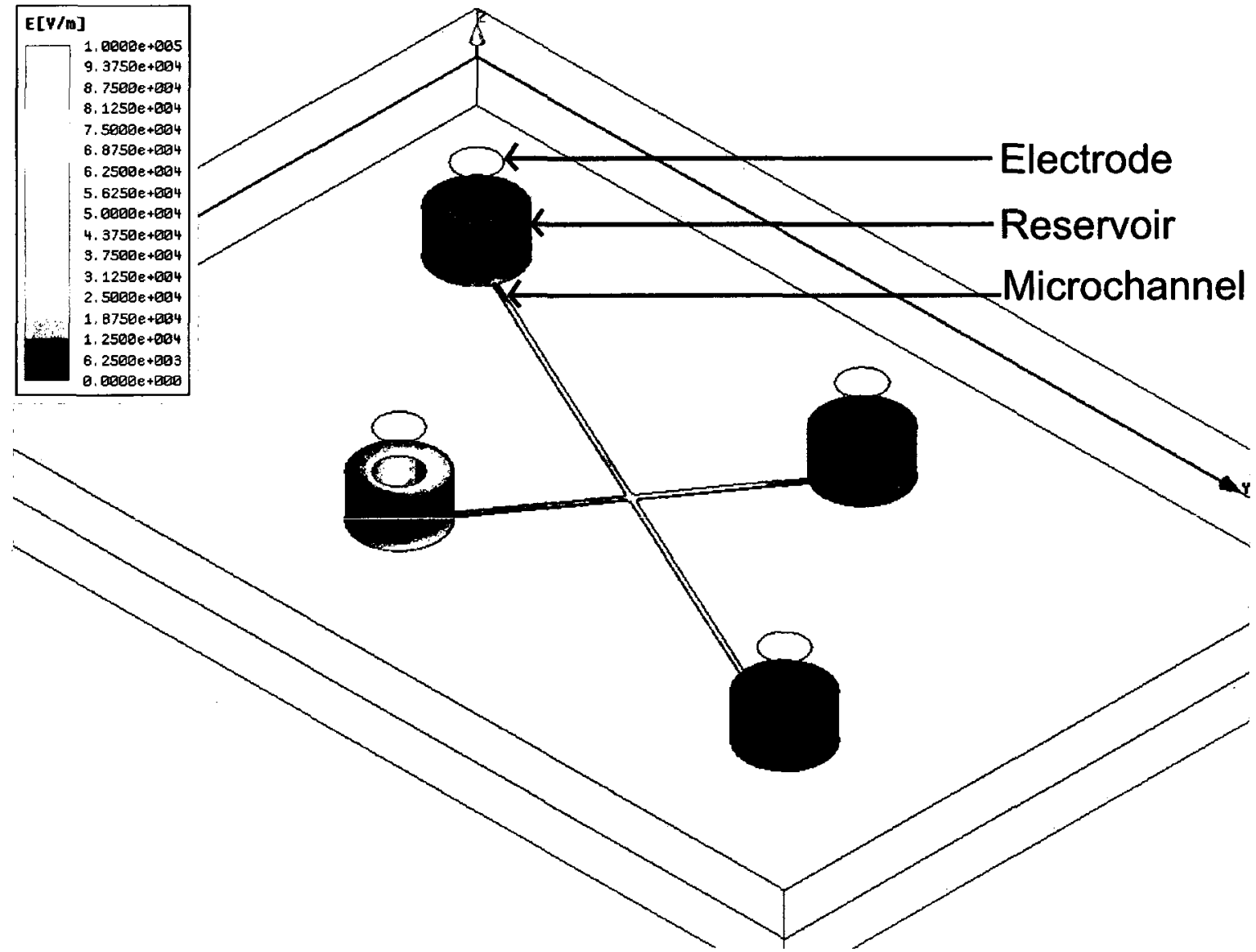

Figure 4.2 FEM simulation of the MFD with microchannel structures. Here electric field inside the microchannel sub-domain is only shown. 
Note that the electric field strength in the reservoirs are very small compared to that in the microchannels.

\subsubsection{Background and rationale of the analysis}

Although the electric field inside a microchannel can be described using Poisson equation [28], to determine the electric field strength inside a microchannel, the parallelplate capacitor electric field equation is commonly used $[47,65,66,97,127]$ :

$$
E=\delta V / \delta l
$$

where $E$ is the electric field, $V$ is the applied potential and $l$ is the distance between electrodes. This ideal case expression, however, is derived for parallel electrodes with uniform electric fields in between and assumes no fringing occurs. To determine a nonuniform electric field distribution inside a microchannel, FEM analysis is preferred that provides near realistic results $[66,108]$. As FEM analysis is time consuming and computationally expensive, a few attempts were made to develop analytical expressions to calculate electric fields inside the microchannels. All such expressions, however, are design specific $[108,128,129]$.

We report an attempt to validate the ideal case expression (4.1) by simulating a simple model of the a microchannel and to extend the simple model to a practical model of an MFD to estimate the error ranges. Extensions of the analytical expressions of the simple model are suggested to reduce such estimation errors. The approach taken is as follows. First, analytical expressions are developed for the simple model, where microchannels are rectangular boxes. These expressions are validated using an FEM analysis of the simple model with a range of microchannel dimensions. Then, a practical model of the microchannel is analyzed using the same FEM analysis and plotted against previously obtained results of the simple model to determine estimation error ranges. Lastly, extension of the simple model expressions are suggested that will minimize estimation errors. 


\subsubsection{Ideal model of a microchannel}

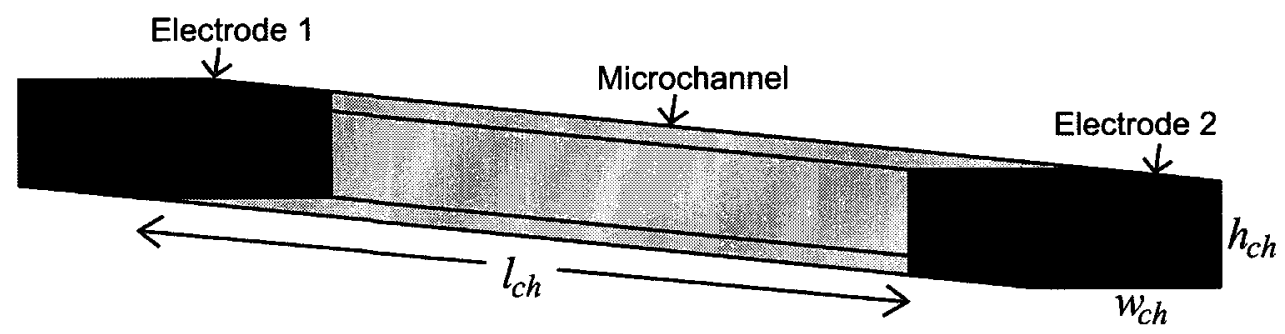

Figure 4.3 A simple model of a rectangular box microchannel filled with D-PBS buffer fluid and electrodes on both sides. The whole structure is enclosed within a glass substrate (not shown).

To formulate an ideal model of a microchannel-based system for developing an electric field, a simple model of a microchannel structure is considered in Figure 4.3. In this model, the microchannel is a rectangular box with length, width and height of $l_{c h}, w_{c h}$ and $h_{c h}$, respectively. Two electrodes on both ends of the microchannel contact the complete cross-sectional areas. The microchannel is filled with a material of relative permittivity and conductivity as Dulbecco's Phosphate Buffered Saline (DPBS) buffer fluid. D-PBS has a relative permittivity $\left(\epsilon_{r}\right)$ and a conductivity $\left(\sigma_{c h}\right)$ of 80 and $1.6 \Omega^{-1} m^{-1}$, respectively [66]. The whole structure is enclosed within a glass substrate, whose conductivity is negligible when compared to D-PBS.

\subsubsection{Analytical expression for the ideal model}

An electric field $\left(E_{c h}\right)$ is developed inside the microchannel (Figure 4.3) as an excitation voltage $\left(V_{a p p}\right)$ is applied across the electrodes. The voltage across the microchannel $\left(V_{c h}\right)$ is the same as $V_{a p p}$ for this simple model. For this case, the electric flux inside the microchannel can be assumed to be uniform with no fringing because of the glass enclosing. Thus, $E_{c h}$ can be expressed using the ideal case expression between two parallel electrodes [130], as

$$
E_{c h}=\frac{V_{c h}}{l_{c h}}
$$


Due to the current flow through the buffer fluid, the (ohmic) power dissipation $\left(P_{d}\right)$ inside the microchannel can be expressed as [131],

$$
P_{d}=\frac{V_{c h}^{2}}{R_{c h}}
$$

where $R_{c h}$ is the electrical resistance of the microchannel. Using the resistivity law [131], $R_{c h}$ for the rectangular-box can be expressed as

$$
R_{c h}=\rho_{c h} l_{c h} / A_{c h}
$$

where $\rho_{c h}\left(=1 / \sigma_{c h}\right)$ is the resistivity of the buffer fluid inside the microchannel, $\sigma_{c h}$ is the corresponding conductivity, and $A_{c h}\left(=w_{c h} \times h_{c h}\right)$ is the cross-sectional area of the microchannel. Using these values in expression (4.3),

$$
P_{d}=\frac{V_{c h}^{2}}{\rho_{c h}}\left(\frac{A_{c h}}{l_{c h}}\right)=\frac{E_{c h}^{2}}{\rho_{c h}} \mathbf{V}_{c h}
$$

where $\mathbf{V}_{c h}\left(=A_{c h} \times l_{c h}\right)$ is the volume of the microchannel. Hence, for a given electric field, the power dissipation is proportional to the volume of the microchannel.

Again, the energy density, $u_{c h}$, stored inside the microchannel due to the electric field can be expressed by [130],

$$
u_{c h}=\frac{1}{2} \epsilon E_{c h}^{2}=\frac{\epsilon}{2} \frac{V_{c h}^{2}}{l_{c h}^{2}}
$$

Here $\epsilon\left(=\epsilon_{0} \epsilon_{r}\right)$ is the permittivity of the buffer fluid, where $\epsilon_{0}$ is the permittivity of the free space and $\epsilon_{r}$ is the relative permittivity of the buffer fluid. The total energy stored $\left(U_{c h}\right)$ within the microchannel can be obtained by integrating $u_{c h}$ over $\mathbf{V}_{c h}$ [130]. For the rectangular box, $U_{c h}$ can be expressed by the simple expression,

$$
U_{c h}=\int_{\mathbf{V}_{c h}} u_{c h} d \mathbf{V}=\frac{1}{2} \epsilon E_{c h}^{2} \mathbf{V}_{c h}=\frac{\epsilon}{2} V_{c h}^{2}\left(\frac{A_{c h}}{l_{c h}}\right) .
$$

Again, for a given electric field, the total energy stored is proportional to the volume of the microchannel. From expressions (4.7) and (4.5), $U_{c h}$ is proportional to $P_{d}$ with a proportionality constant of $\epsilon \rho_{c h} / 2$. 
These analytical expressions (4.2,4.5-4.7) for the simple model are plotted in Figure 4.4 to Figure 4.7 (as the solid lines) for a range of $V_{a p p}$. In Figure 4.4 and Figure 4.6, plots of the electric field strengths and the energy densities are plotted against the lengths of the microchannels $\left(l_{c h}\right)$ using Expression (4.2) and Expression (4.6), respectively. Figure 4.5 shows the power dissipation curves against the area-over-length ratio $\left(A_{c h} / l_{c h}\right)$ using Expression (4.5). Figure 4.7 shows the relationship of the total energy stored inside the microchannel against $A_{c h} / l_{c h}$ using Expression (4.7).

\subsubsection{Simulation of an ideal microchannel model}

An ideal (simple) microchannel model (Figure 4.3) was simulated for a range of microchannel dimensions by using an FEM analysis tool Maxwell 3-D (3 dimensional) simulator from Ansoft Corporation. A script file was written for this purpose to simulate 175 different sets of dimensions $\left(l_{c h}, w_{c h}\right.$ and $\left.h_{c h}\right)$. The range of $l_{c h}, w_{c h}$ and $h_{c h}$ simulated were 100 to $10000 \mu \mathrm{m}, 1$ to $1000 \mu \mathrm{m}$, and 10 to $1000 \mu \mathrm{m}$, respectively. Each structure was simulated for excitation voltages ranging from $1 \mathrm{~V}$ to $1000 \mathrm{~V}$. The material property for the buffer fluid inside the microchannel was set to match D-PBS property $\left(\epsilon_{r}=80\right.$ and $\left.\sigma_{c h}=1.6 \Omega^{-1} m^{-1}\right)$. The resultant data were collected, analyzed, and plotted using Matlab in Figure 4.4 to Figure 4.7. The average of these data are represented by dots (.), and the standard errors are denoted by the error-bars on both sides of the mean.

To represent the measure of dependency of the data points from simulations on the plotted $\mathrm{x}$-axis variables, we have used the metric normalized standard errors. The normalized standard errors are calculated by dividing the standard errors by the corresponding means. The maximum values of the normalized standard errors are tabulated in Table 4.1. The small values of these normalized standard errors confirm that the results are primarily dependent on the $\mathrm{x}$-axis variables. 


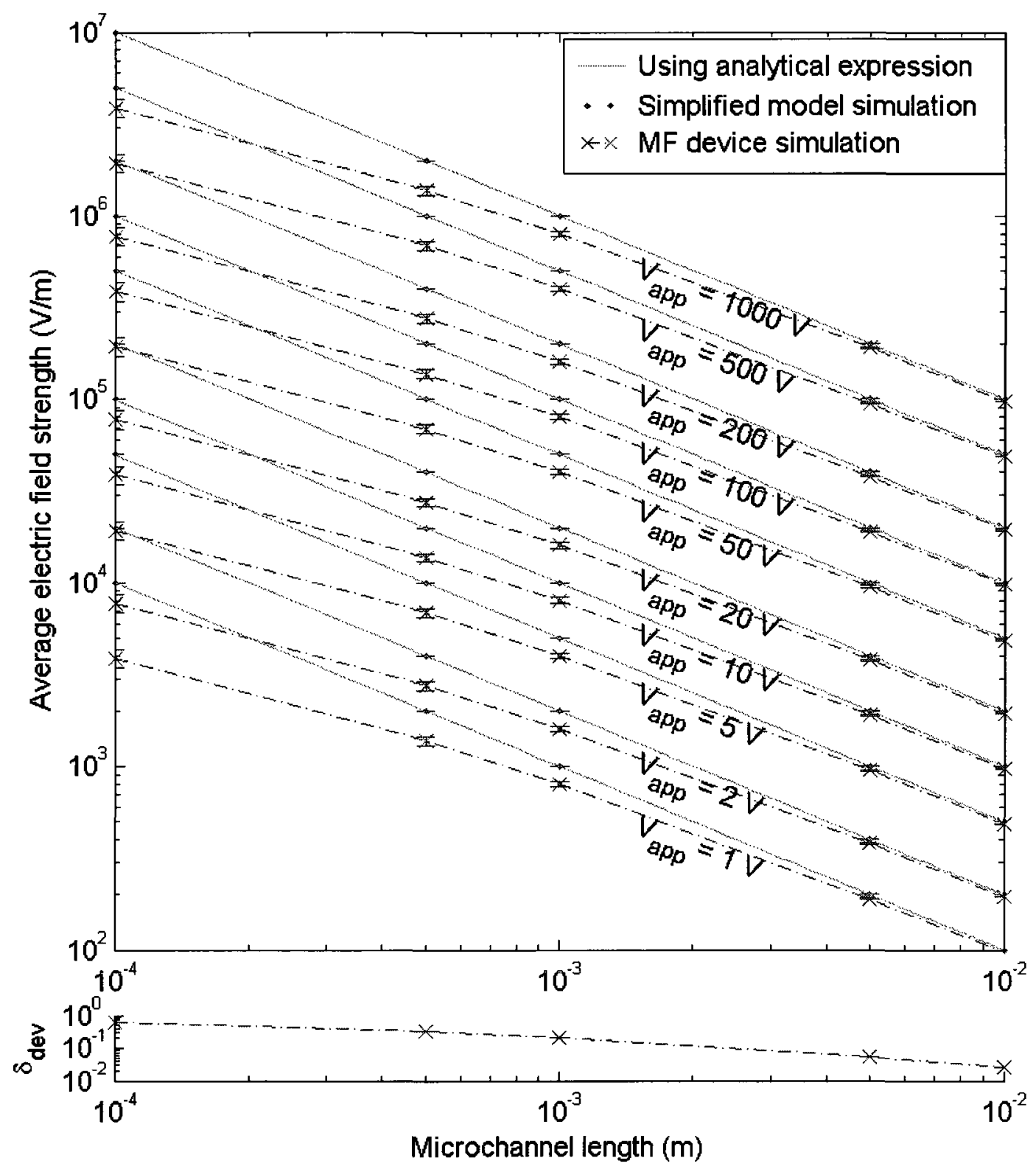

Figure 4.4 Plot representing relationship between the developed electric field strengths $\left(E_{c h}\right)$ with the lengths of the microchannels $\left(l_{c h}\right)$ for various excitation voltages $\left(V_{a p p}\right)$. Normalized deviations $\left(\delta_{d e v}\right)$ are plotted at the bottom. All simulation results include error bars representing standard errors. 


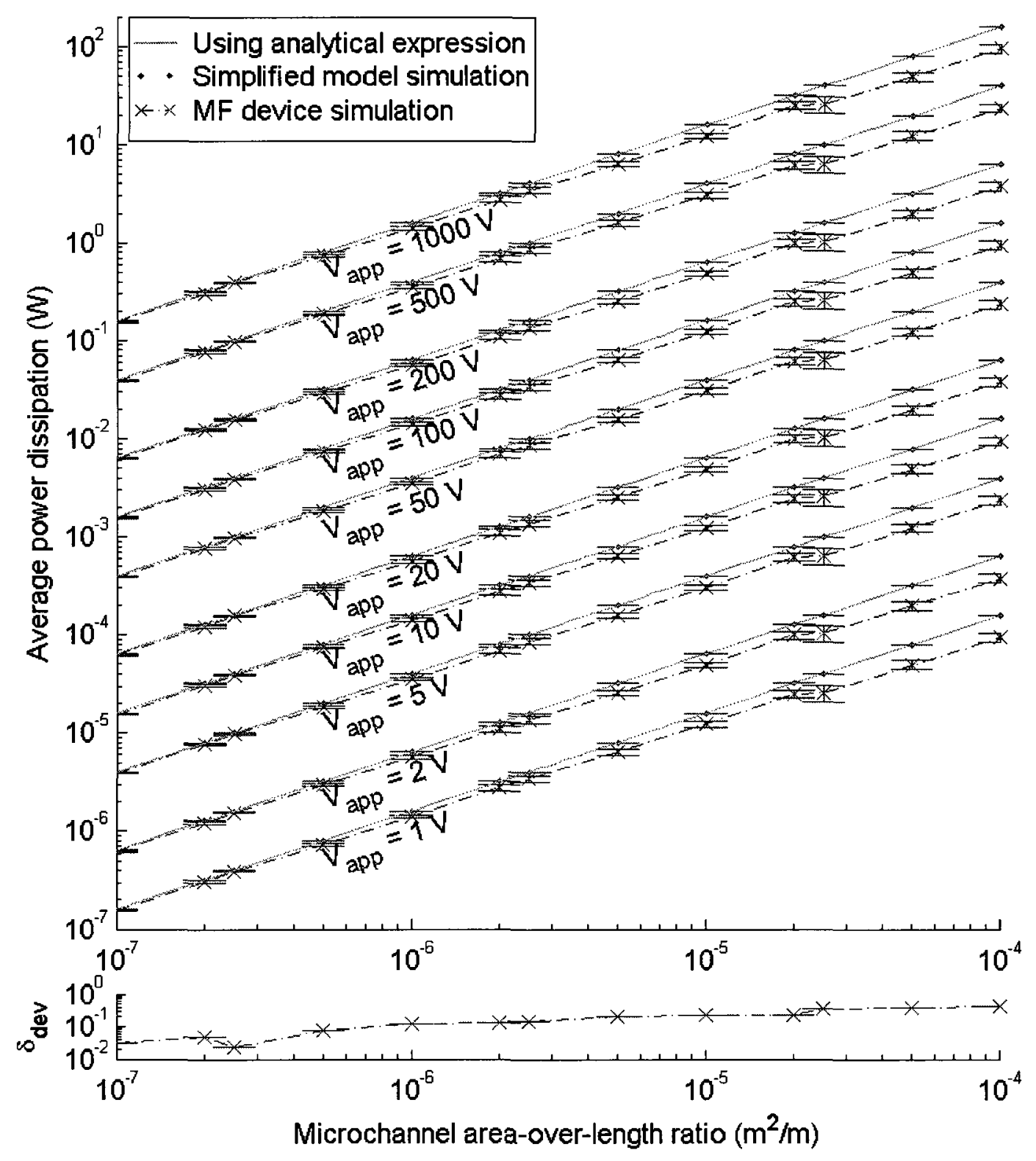

Figure 4.5 Plot depicting power dissipations $\left(P_{d}\right)$ for various area-overlength ratios $\left(A_{c h} / l_{c h}\right)$ of the microchannels for various excitation voltages $\left(V_{a p p}\right)$. Here, $\rho_{c h}=0.625 \Omega-\mathrm{m}$. Normalized deviations $\left(\delta_{\text {dev }}\right)$ are plotted at the bottom. All simulation results include error bars representing standard errors. 


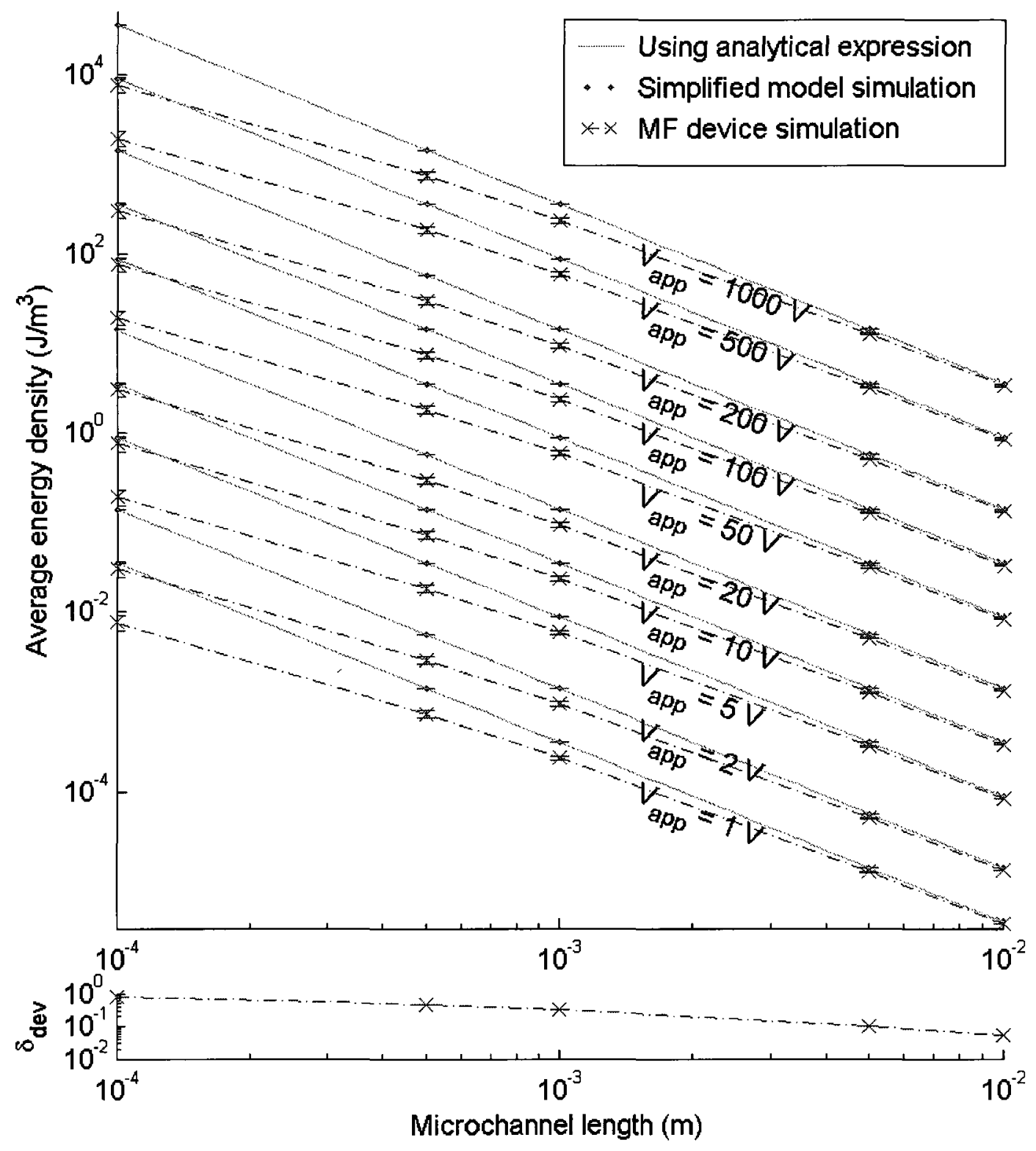

Figure 4.6 Plot showing dependance of the energy densities $\left(u_{c h}\right)$ inside the microchannels with the microchannel lengths $\left(l_{c h}\right)$ for various excitation voltages $\left(V_{a p p}\right)$. Here, $\epsilon_{r}=80$. Normalized deviations $\left(\delta_{\text {dev }}\right)$ are shown at the bottom. All simulation results include error bars representing standard errors. 


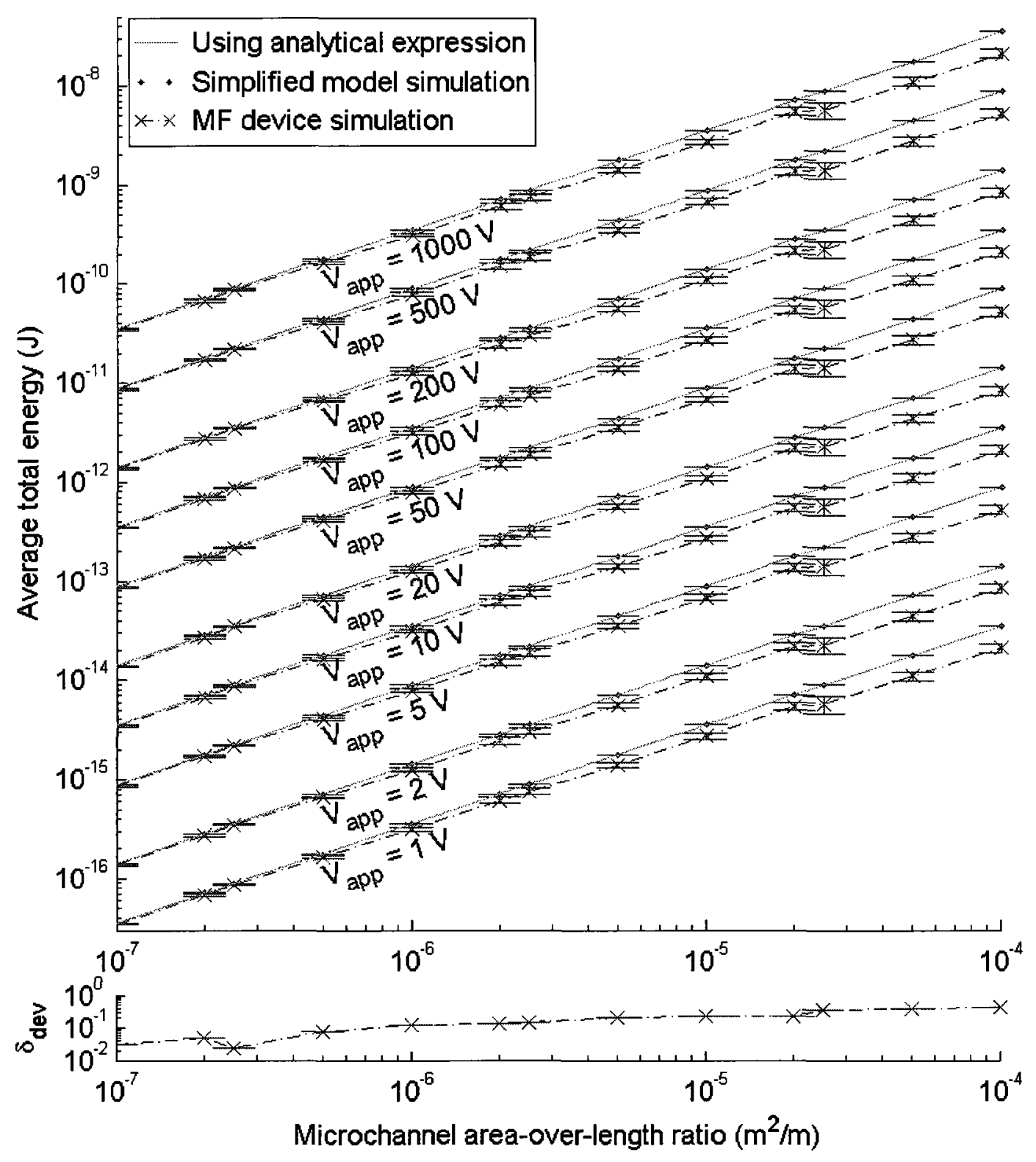

Figure 4.7 Plot representing the total energies $\left(U_{c h}\right)$ stored versus area-overlength ratios $\left(A_{c h} / l_{c h}\right)$ of the microchannels for various excitation voltages $\left(V_{a p p}\right)$. Here, $\epsilon_{r}=80$. Normalized deviations $\left(\delta_{d e v}\right)$ are given at the bottom. All simulation results include error bars representing standard errors. 


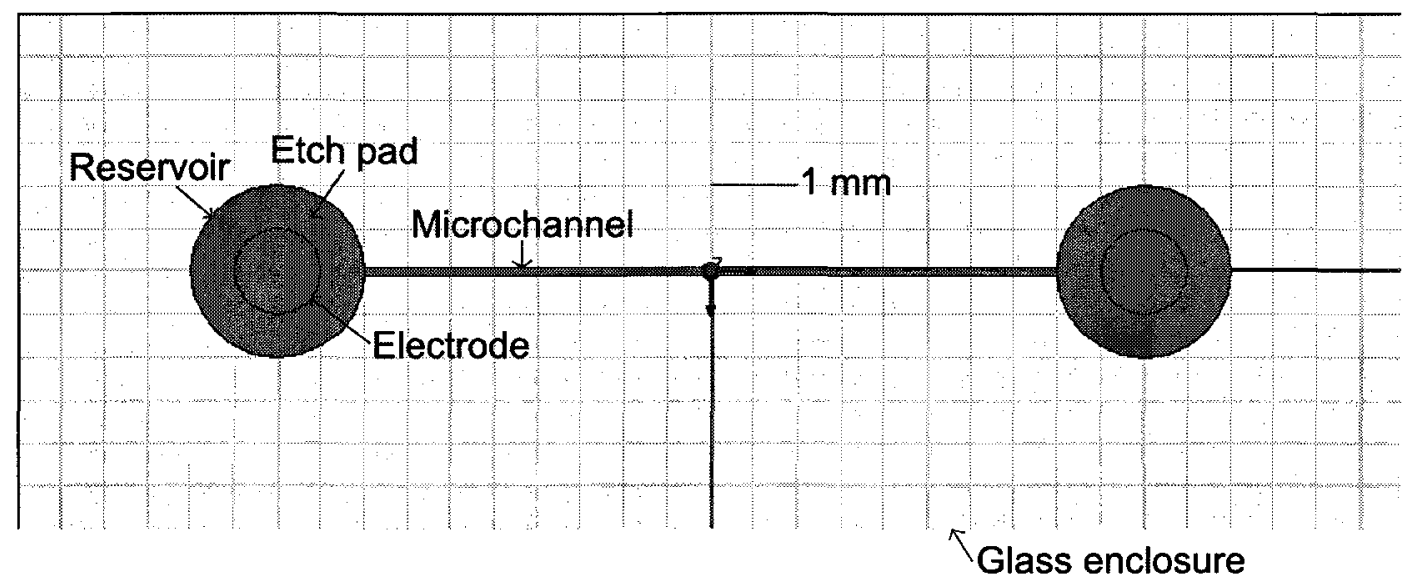

Figure 4.8 Top view of a microchannel of the MFD to be analyzed for developed EFs.

\subsubsection{Model of a microchannel with external electrodes in reservoirs}

Figure 4.8 shows the top view of a microchannel of the MFD joining two access holes (reservoirs). To develop an electric field inside the microchannel, electrodes are inserted inside these reservoirs (mimicking the method used). The microchannel and the reservoirs were filled with a D-PBS buffer fluid using proper conductivity and permittivity values (as mentioned before). The electrodes were excited with the applied voltage, $V_{a p p}$. Note that in this case, $V_{c h}<V_{a p p}$. This setup is depicted in Figure 4.9 using a 3-dimensional (3-D) drawing of a portion of the microchannel.

\subsubsection{Simulations of the microchannel with external elec- trodes}

The MFD model (shown in Figure 4.8) was simulated using another script file with the same FEM analysis tool. Dimensions of the microchannel were varied for the same ranges as the simple model, while the dimensions of the reservoirs, electrodes, and etch holes were kept constant. The electrodes of a $1 \mathrm{~mm}$ diameter were positioned at the middle of the reservoirs, $0.1 \mathrm{~mm}$ above the bottom plate. The number of iterations 


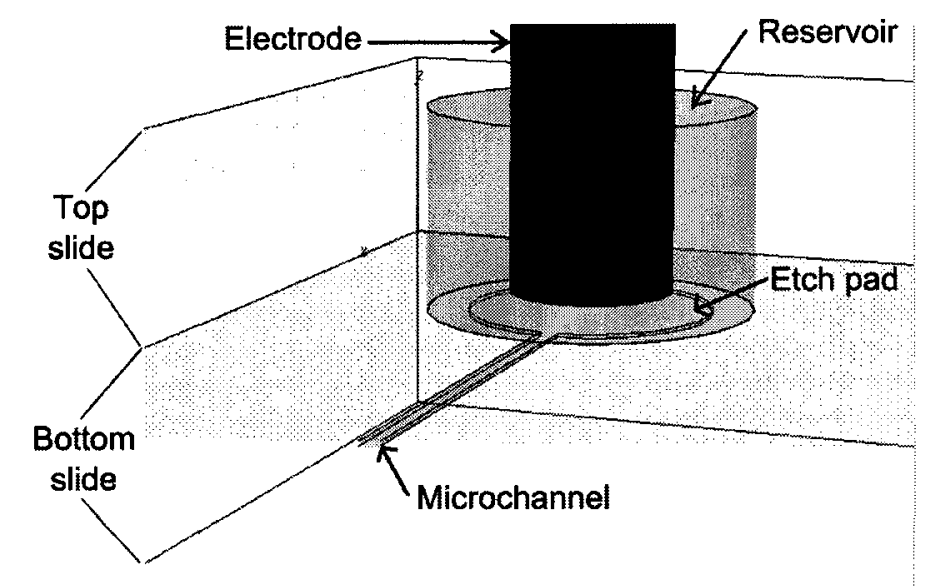

Figure 4.9 A 3-D view of a portion of the microchannel of the MFD shown in Figure 4.8 is depicted here. The top and bottom glass-slides that compose the glass enclosure, an electrode, an access hole, an etch-pad and a section of a microchannel are indicated.

performed were kept constant to 20 . The resultant data were collected, analyzed, and plotted against previous results in Figure 4.4 to Figure 4.7. The mean of the data are denoted by crosses $(x)$, and the standard errors are denoted by the error-bars on both sides of the means. The mean values are connected with dot-dashed lines to show the trend.

The maximum values of the normalized standard errors for this MFD (Table 4.1) are higher compared to those of the simple model, but were below 0.3. Hence over $60 \%$ data are confined within $30 \%$ of mean data. These high values of normalized standard errors indicate that the $y$-axis results are influenced by additional variables than the $\mathrm{x}$-axis variables. One such additional variables is the relative dimension of the reservoirs.

The mean data of the MFD simulation results deviate from those of the simple model, especially for small $l_{c h}$ (i.e. large $A_{c h} / l_{c h}$ ). This deviations, resulting estimation errors, should be taken under consideration for any electric field related applications. This is especially important for small lengths of microchannels as reported in some literatures $[65,66,127]$. To quantify these deviations, the normalized deviations $\left(\delta_{d e v}\right)$, that gives a measure of normalized covariances, are plotted beneath 
Table 4.1 Maximum values of the normalized standard errors from FEM simulations of the simple model and the MFD model

\begin{tabular}{lll} 
Plot & Simple model & MFD model \\
\hline$E_{c h}$ vs $l_{c h}$ & 0 & 0.1145 \\
$P_{d}$ vs $A_{c h} / l_{c h}$ & $9.8 \times 10^{-17}$ & 0.2997 \\
$u_{c h}$ vs $l_{c h}$ & $1.7 \times 10^{-7}$ & 0.1905 \\
$U_{c h}$ vs $A_{c h} / l_{c h}$ & $5.65 \times 10^{-7}$ & 0.2997
\end{tabular}

each plots (Figure 4.4 to Figure 4.7 ). Here, $\delta_{d e v}$ are calculated by subtracting the mean data for the MFD from the mean data for the simple model, then dividing it by the mean data for the simple model. Mathematically,

$$
\delta_{d e v}=\frac{E_{i}-E_{c h}}{E_{i}}
$$

where $E_{i}$ is the electric field calculated using Expression (4.2) for simple model and $E_{c h}$ is the electric field developed inside the microchannel of the MFD from simulation results. The resultant $\delta_{d e v}$ are averaged for various $V_{a p p}$. The mean values of these averages are shown using crosses $(x)$, while the standard errors are shown as the error bars in these $\delta_{\text {dev }}$ plots. Again, smaller values of the standard errors indicate that $\delta_{d e v}$ is not dependent on $V_{a p p}$.

The values of $\delta_{\text {dev }}$ are positive, indicating the analytical expressions always overestimate. This is because $V_{c h}$ is always smaller than $V_{a p p}$ in microchannels of an MFD. As microchannel lengths become smaller (i.e. area-over-length ratios become greater), the difference between $V_{c h}$ and $V_{a p p}$ increases, thus $\delta_{d e v}$ value also increases. The amount of overestimation are tabulated in Table 4.2 for the range of $10 \%$ and $50 \%$ overestimation. Thus, one can rationalize that these estimation errors resulted from the presence of the reservoirs in the MFD. These estimation errors can be minimized by determining $V_{c h}$ for the MFD. To calculate $V_{c h}$, one has to determine the resistance introduced by the reservoirs. Analytical expression of such structures to 
Table 4.2 The range of variables for the estimation error ranges when calculating various parameters (electric field, power dissipation, energy density and total energy stored) for the microchannel-based MFDs using the derived analytical expressions

\begin{tabular}{lll} 
Parameter & $\mathbf{1 0 \%}$ estimation error & $\mathbf{5 0 \%}$ estimation error \\
\hline$E_{c h}$ & $l_{c h}=2300 \mu \mathrm{m}$ & $l_{c h}=175 \mu \mathrm{m}$ \\
$P_{d}$ & $\frac{A_{c h}}{l_{c h}}=0.7 \mu \mathrm{m}^{2} / \mu \mathrm{m}$ & $\frac{A_{c h}}{l_{c h}}=100 \mu \mathrm{m}^{2} / \mu \mathrm{m}$ \\
$u_{c h}$ & $l_{c h}=5000 \mu \mathrm{m}$ & $l_{c h}=400 \mu \mathrm{m}$ \\
$U_{c h}$ & $\frac{A_{c h}}{l_{c h}}=0.7 \mu \mathrm{m}^{2} / \mu \mathrm{m}$ & $\frac{A_{c h}}{l_{c h}}=100 \mu \mathrm{m}^{2} / \mu \mathrm{m}$
\end{tabular}

determine resistances is tedious, and dependent on the particular shape and dimensions of the reservoirs. Extensions of the analytical model are proposed in the next section to reduce the estimation error.

\subsubsection{Extended analytical model to reduce estimation errors}

From $\delta_{d e v}$ plots, one can conclude that dependance of $\delta_{d e v}$ on variation of $V_{a p p}$ is negligible, evident by the small values (less than 1\%) of standard errors (represented by error bars in the $\delta_{\text {dev }}$ plots). The reservoirs in the MFD model cause certain voltage drops inside the reservoirs, resulting $V_{c h}<V_{a p p}$. As the channel length decreases, the voltage drop across the reservoirs become increasingly significant. To formulate this, a resistance model of the setup can be used, where the microchannel resistance $\left(R_{c h}\right)$ is in series with the two reservoir resistances $\left(R_{\text {res }}\right)$. The applied potential $\left(V_{a p p}\right)$ causes a potential $\left(V_{c h}\right)$ to be developed across the channel. Hence, the electric field 
inside the microchannel $\left(E_{c h}\right)$ of the MFD can be given as:

$$
\begin{aligned}
E_{c h} & =\frac{V_{c h}}{l_{c h}} \\
& =\frac{V_{a p p}-2 I_{c h} R_{r e s}}{l_{c h}} \\
& =\frac{V_{a p p}}{l_{c h}}-\frac{2 V_{a p p} R_{r e s}}{\left(R_{c h}+2 R_{r e s}\right) l_{c h}} \\
& =\frac{V_{a p p}}{l_{c h}}-\frac{V_{a p p}}{l_{c h}} \frac{1}{1+\alpha} \\
& =\frac{V_{a p p}}{l_{c h}}\left(1-\frac{1}{1+\alpha}\right)
\end{aligned}
$$

where $\alpha=R_{c h} / 2 R_{\text {res }}$ is the ratio of channel resistance to total reservoir resistances.

For large $l_{c h}, R_{c h}$ is much higher than $R_{\text {res }}$. Hence, $\alpha \gg 1$, resulting $1 /(1+\alpha) \approx 0$. Thus $E_{c h} \approx V_{a p p} / l_{c h}$. For very small $l_{c h}, R_{c h}$ is much smaller than $R_{r e s}, \alpha \ll 1$ and $1 /(1+\alpha) \approx 1$. In this case, $E_{c h} \approx 0$.

Comparable expressions can be reached by analyzing the plotted graphs. From Figure 4.4, approximating a linear relationship between $\log \left(\delta_{d e v}\right)$ and $\log \left(l_{c h}\right)$, we get:

$$
\begin{array}{rll}
\log \left(\delta_{\text {dev }}\right) & =-m \log \left(l_{c h}\right)+\log (c) \\
\Rightarrow \quad \log \left(\delta_{\text {dev }}\right) & =\log \left(l_{c h}^{-m}\right)+\log (c) \\
\Rightarrow \quad \delta_{\text {dev }} & =c l_{c h}^{-m} \\
\Rightarrow \quad \frac{E_{i}-E_{c h}}{E_{i}} & =\frac{c}{l_{c h}^{m}} \\
\Rightarrow \quad E_{c h} & =E_{i}\left(1-\frac{c}{l_{c h}^{m}}\right) \\
\Rightarrow \quad E_{c h} & =\frac{V_{a p p}}{l_{c h}}\left(1-\frac{c}{l_{c h}^{m}}\right)
\end{array}
$$

where $m$ is the slope of the curve, $c$ is a constant, $E_{i}$ is the electric field calculated from the simple model, and $E_{c h}$ is the simulation results of the electric field developed inside the microchannel. Comparing Expression (4.9) with Expression (4.10), $l_{c h}^{m} / c=1+\alpha$. Value of $\alpha$ can be determined by determining $R_{c h}$. This extension of analytical expression will reduce estimation errors as the mean of estimation errors are incorporated. Similarly, extensions to other expressions can be derived to minimize $\left|\delta_{d e v}\right|$. 


\subsubsection{Simulation of the initial device}

FEM simulations of the MFD using the specific dimensions were conducted to verify specific results. As a case study, an $8 \mathrm{~mm}$ long microchannel simulation results are shown in Figure 4.10. The developed electric field inside the microchannel remains uniform throughout the length. The magnitude of this field can be approximated by using expression (4.2) without significant estimation error (less than 5\%). The electric field developed inside the microchannel due to the application of $800 \mathrm{~V}$ through the electrode-pair is about $100 \mathrm{kV} / \mathrm{m}$, whereas the electric field inside the reservoirs are negligible compared to that in the microchannel, as depicted in the figure. Hence, for the fabricated device, the developed expressions of the simple model can be safely used to estimated the electric field inside the microchannels.

To determine the effect of the locations of electrodes inside the reservoirs on the developed electric field, the MFD is simulated with electrodes moved at different locations within reservoirs. The results are summarized in Figure 4.11. The electric field inside the microchannel (about $125 \mathrm{~V} / \mathrm{m}$ for one volt of excitation voltage) is unaffected by such change of locations of the electrodes inside the reservoirs at five different locations (location 1-1 to location 5-5 as shown in Figure 4.11). Furthermore, Figure 4.11 shows that the voltage linearly drops inside the microchannel only, and almost no voltage drop occurring inside the reservoirs. Hence, it can be concluded that the electrical resistance developed inside the reservoirs are negligible when compared to that inside the microchannel for this microchannel length.

From our analysis, more than $10 \%$ overestimation of the electric field results for the MFD with microchannel lengths smaller than $2.5 \mathrm{~mm}$. These analysis and plots are applicable to any MFD that share the same topology as the one analyzed in this section $[47,65,66,97]$. Note that, none of the analysis takes account of the effect of moving charges of ionic fluids, which limits the accuracy to some extent.

To develop an electric field of $100 \mathrm{kV} / \mathrm{m}$ with a $8 \mathrm{~mm}$ long microchannel filled with D-PBS buffer fluid, the required voltage $\left(V_{a p p}\right)$ is $800 \mathrm{~V}$ (Figure 4.4). From 


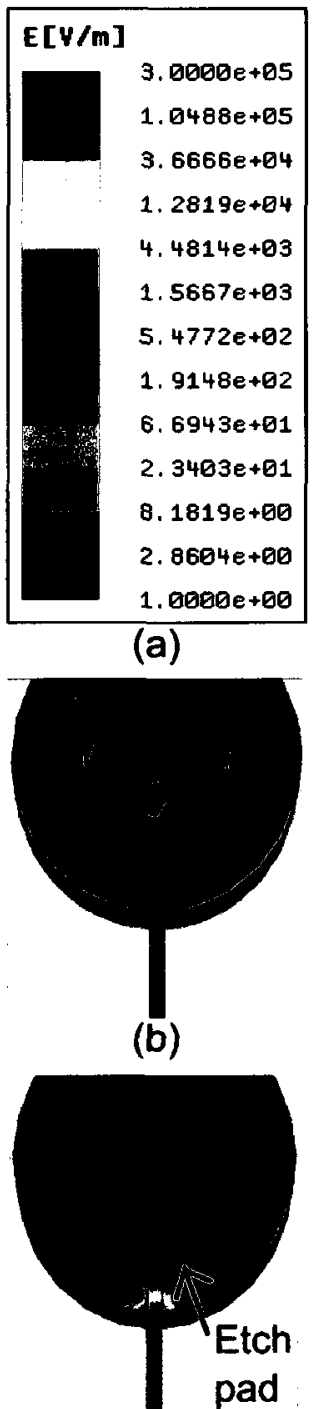

(c)

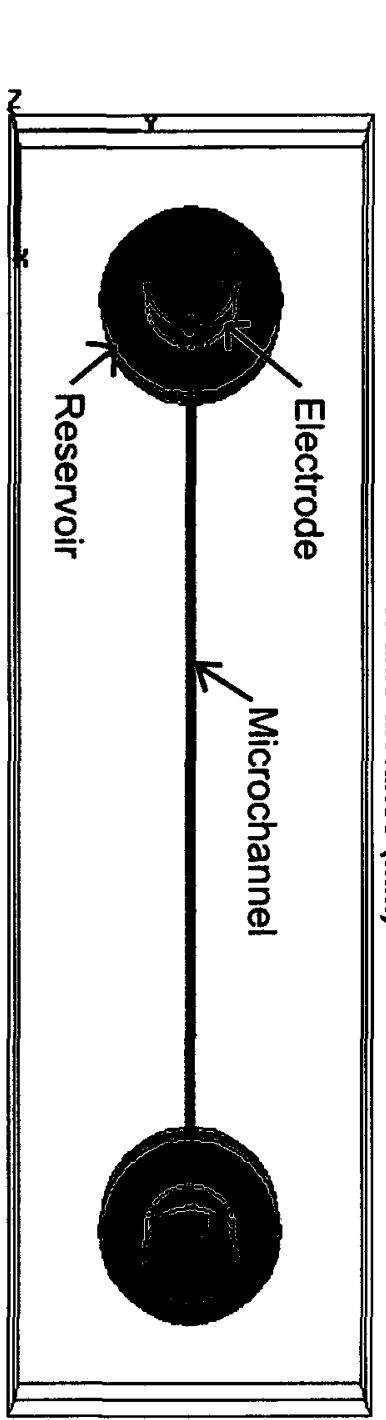

(d)

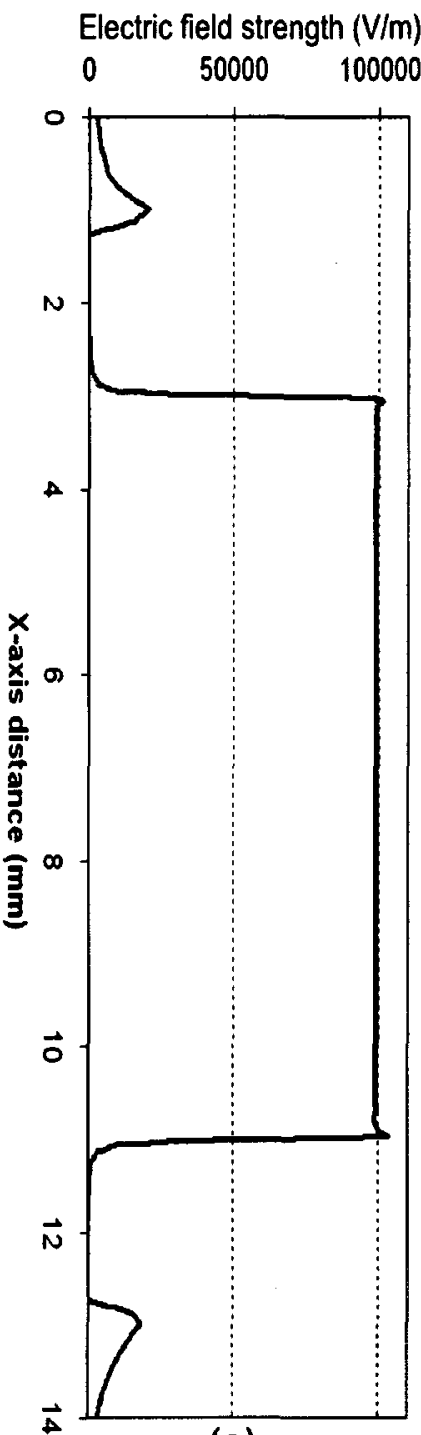

(e)

Figure 4.10 FEM simulation of a $8 \mathrm{~mm}$ long microchannel of the MFD for an applied voltage of $800 \mathrm{~V}$. The microchannel width and height are $100 \mu \mathrm{m}$ and $20 \mu \mathrm{m}$, respectively. (a) Color key legend of the electric field strengths. (b) and (c) Electric field distribution at the reserver-microchannel intersection (top and bottom view, respectively). (d) Electric field distribution along the microchannel and reservoirs (top view). (e) A plot of the electric field strength at the middle of the microchannel (horizontally aligned with (d)). Here electric field inside the microchannel sub-domain is only shown. 


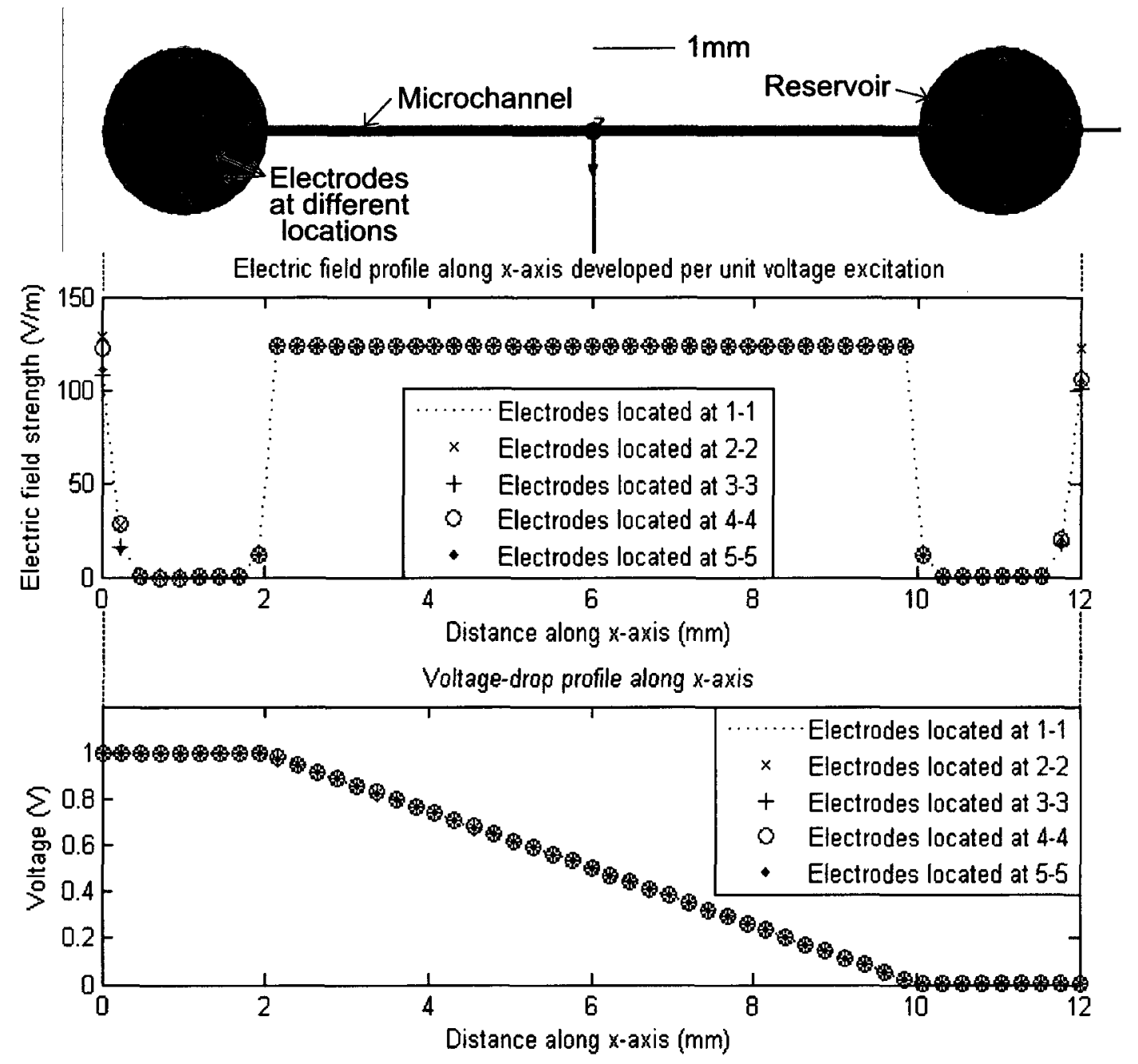

Figure 4.11 Electric field and voltage profile inside the microchannel with electrodes positioned at various locations (location 1-1 to location 5-5) of the reservoirs are shown (vertically aligned) below the model of the MFD. Five images of various locations of electrodes are superimposed in the model. 
Figure 4.6, the energy density $\left(u_{c h}\right)$ corresponding to this length and applied voltage is about $3.5 \mathrm{~J} / \mathrm{m}^{3}$. For a microchannel width of $100 \mu \mathrm{m}$, the cross section area $\left(A_{c h}\right)$ of the microchannel becomes $2 \times 10^{-9} \mathrm{~m}^{2}$, as the height of the microchannel is 20 $\mu \mathrm{m}$, thus the area-over-length ratio $\left(A_{c h} / l_{c h}\right)$ is $2.5 \times 10^{-7} \mathrm{~m}$. The corresponding power dissipation $\left(P_{d}\right)$ for this area-over-length ratio is about $256 \mathrm{~mW}$ (Figure 4.5 ). The total energy stored $\left(U_{c h}\right)$ for the $8 \mathrm{~mm}$ long microchannel calculated using the expression (4.7) is about $567 \mathrm{~mJ}$.

\subsubsection{Electric field simulation of the final device}

Analysis of the developed electric field pattern inside the cell lysis chamber was performed using Comsol Multiphysics (Comsol Inc., Burlington, MA, USA) FEM simulation tool. The model of the device was developed using the built-in 3D modeler of Comsol by using the device dimensions. Figure 4.12 shows the model of the final device created using this modeler for electrostatic simulations to determine electric fields.

The model material parameters were selected from Comsol Multiphysics Material Database component of the tool. The rectangular box substrate (Figure 4.12) was glass, the channel and loading ports were water (liquid) and the electrode materials were platinum.

The boundary conditions for all external surfaces were set to electrical insulation, whereas all internal boundaries were electrical continuity. The positive and negative terminals were excited by applying potential of $20 \mathrm{~V}$ and $0 \mathrm{~V}$, respectively. Isotropic conductivity was chosen for all materials to reduce computational complexity.

The device was meshed using the Comsol predefined "extra fine" meshing to obtain a high spatial resolution. The maximum growth rate was 1.3 resulting 235572 tetrahedron elements with 389124 mesh points and 3089308 degrees of freedom. The meshed structure is shown in Figure 4.13.

Electric field simulation result for $20 \mathrm{~V}$ applied potential along the microchannel 


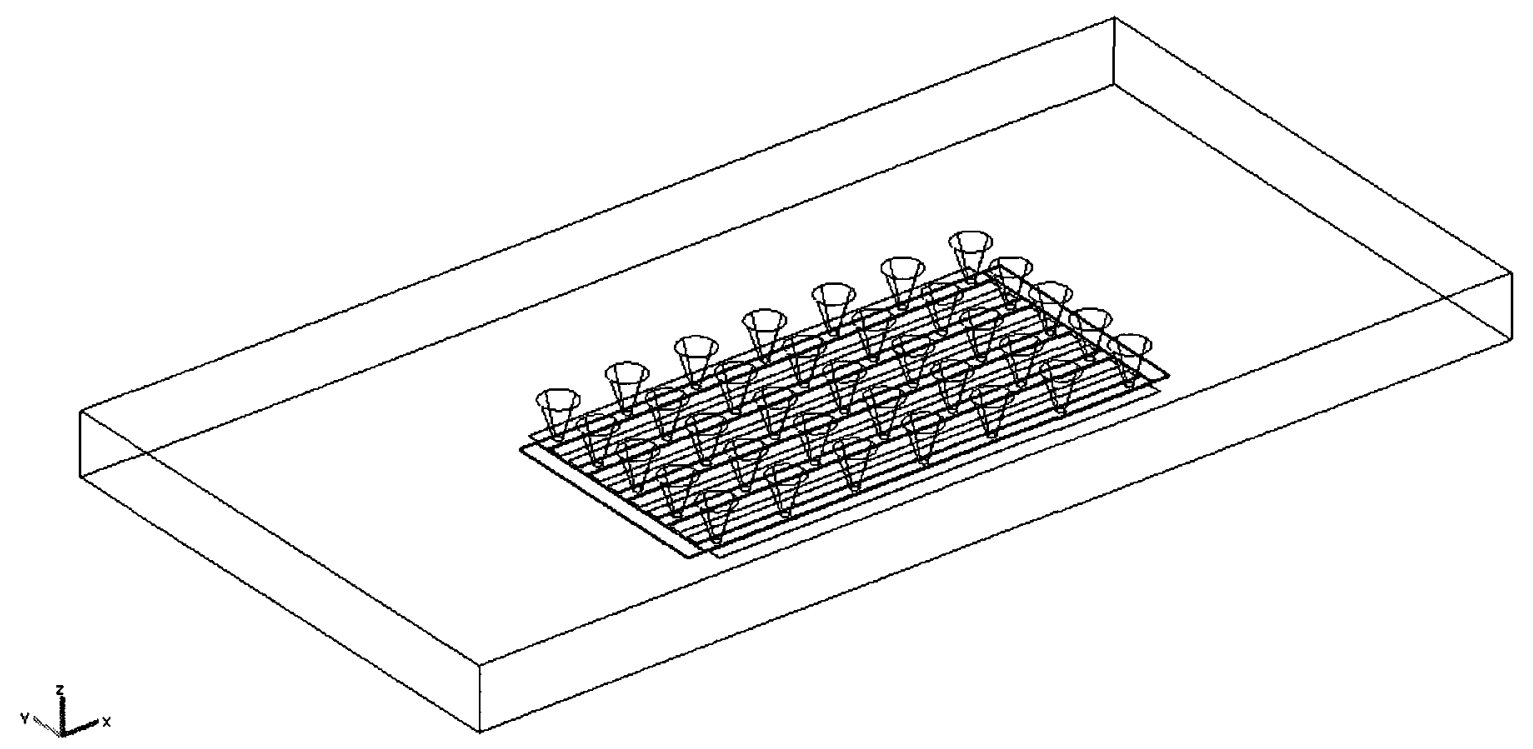

Figure 4.12 A 3D view of the model of the micro-device designed using Comsol Modeler for FEM simulations.

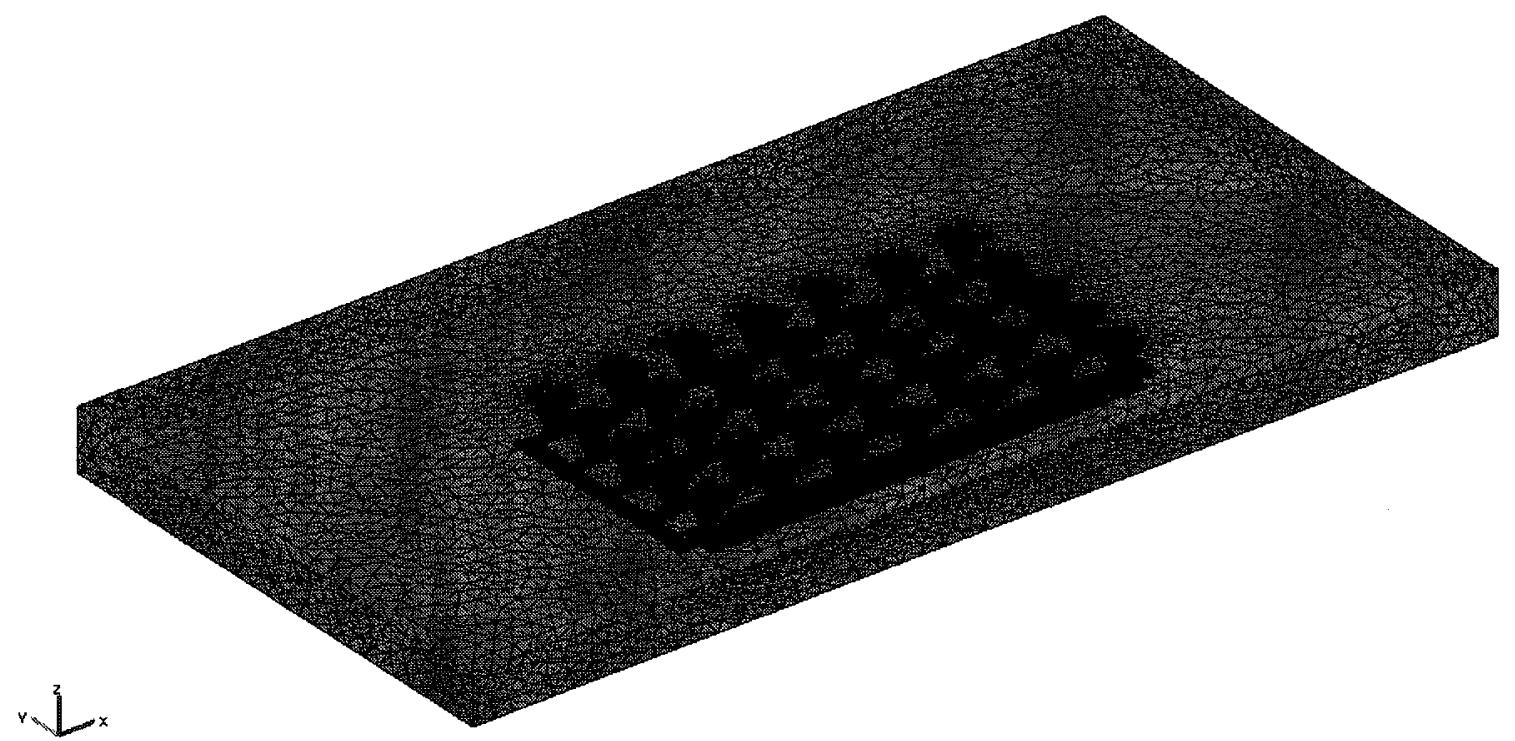

Figure 4.13 Meshed structure of the micro-device for electric field simulation. 
bottom surface (xy-plane) is shown in Figure 4.14. The results show that electric fields up to $2.5 \mathrm{MV} / \mathrm{m}$ were generated in the cell lysis chamber on top of the interdigitated electrodes. Electric field in other regions, where interdigitated electrodes are not present, are low. Electric fields along the cross section of the microchannel (xz-plane) with interdigitated electrodes are shown in Figure 4.15. The figure shows the electric field distribution along the height of the channel. The electric field inside glass in between electrodes were the maximum $(2.5 \mathrm{MV} / \mathrm{m})$. Electric fields decreases along the microchannel height above electrodes. The electric field within the microchannel is maximum at the bottom of the microchannel. This is one of the critical advantage of this device as cells will settle to the bottom of the microchannel after they are loaded in the cell lysis chamber, and will be exposed to a higher electric field, compared to that achievable with electrodes on the sidewalls. Hence this design feature effectively utilizes the developed electric field for EP or EL of sample cells.

The transient development of the electric field has been analyzed using a transient analysis of the model for $10 \mathrm{~s}$ (Figure 4.16) using the software. In the figure, normalized electric field developed at a point which was $1 \mu \mathrm{m}$ above the microchannel bottom surface is plotted. The simulation result demonstrated that the electric field was established within $0.1 \mathrm{~s}$ and was constant at $0.298 \mathrm{MV} / \mathrm{m}$ strength for the complete duration of the transient analysis. The small deflection at the beginning can be attributed to the electric field setup time due to initial transients from a state of zero electric field. However, this initial transient value of electric field was within the same electric field strength up to three decimal points.

\subsection{Thermal Simulation}

Application of electrical current through the buffer fluid causes generation of heat (primarily Joule heating). To determine the rise in temperature due to such heating with the developed microfluidic devices, thermal simulations were conducted. In this section, thermal simulation of the final device is described, as this is the device of 


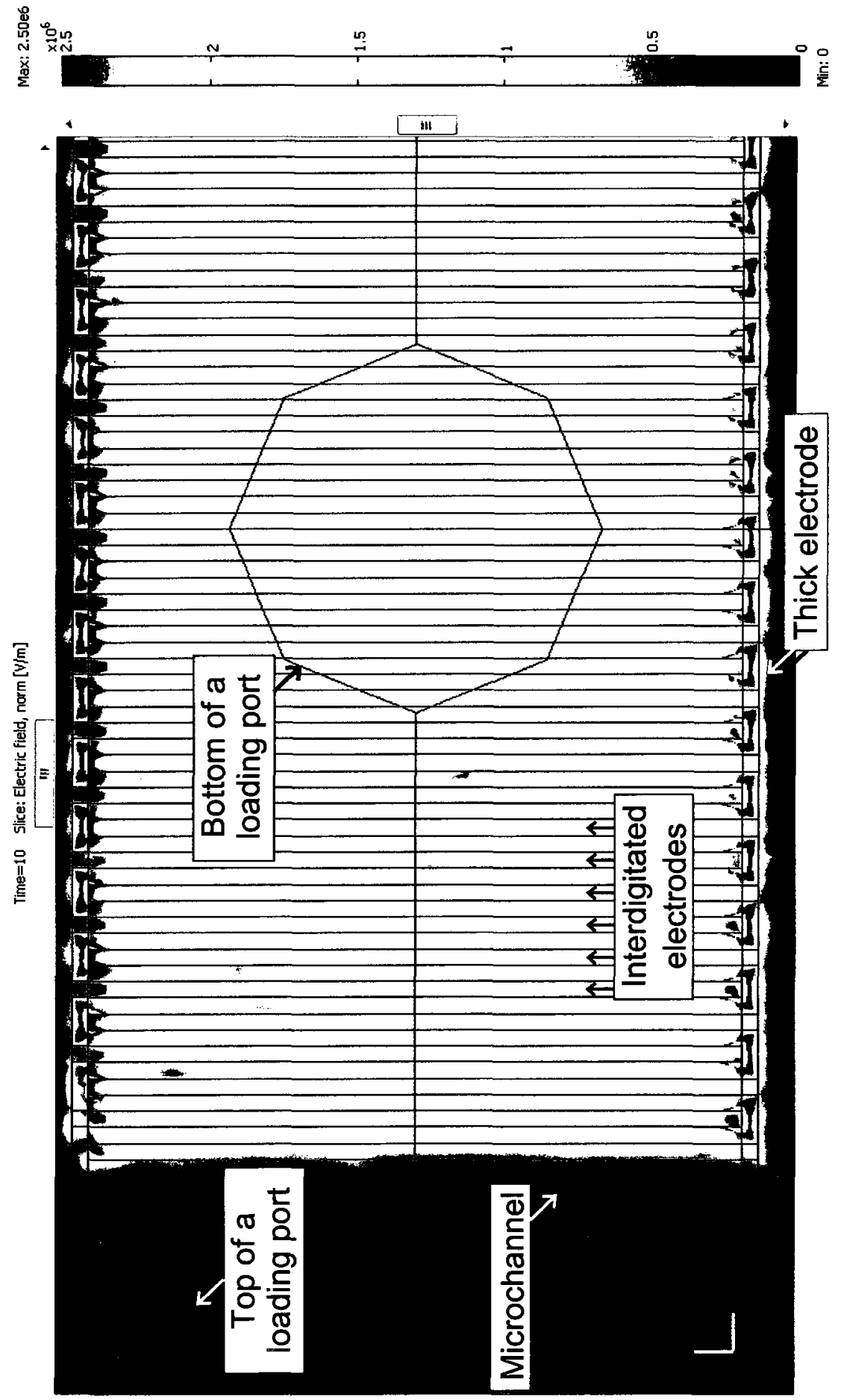

Figure 4.14 Electric field simulation of the final device along xy-plane demonstrating development of fields of $2.5 \mathrm{MV} / \mathrm{m}$ strengths. 


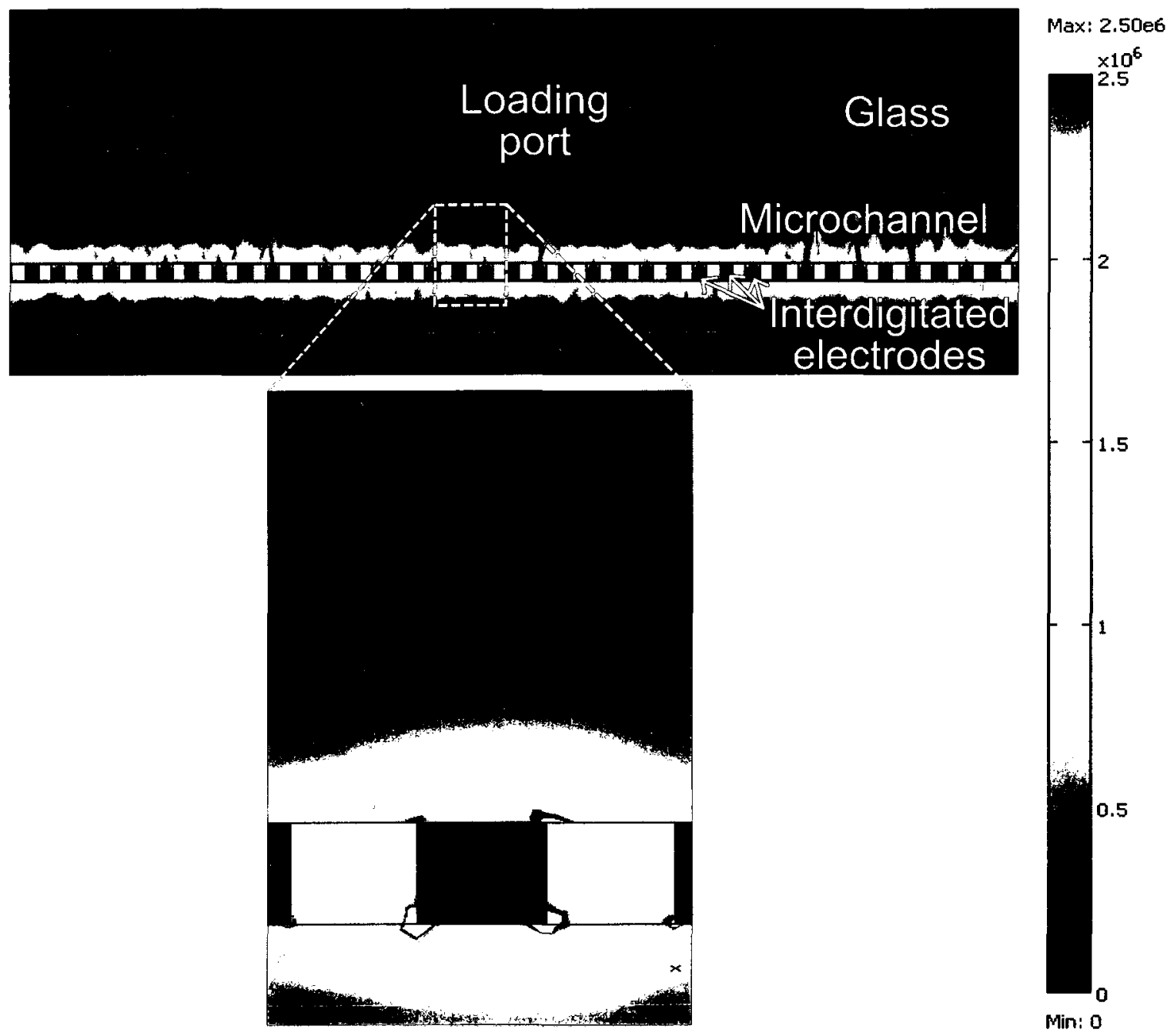

Figure 4.15 Electric field simulation of the final device along xz-plane with a section (with higher mesh size) enlarged to show distribution of electric fields along the height of the microchannel. 


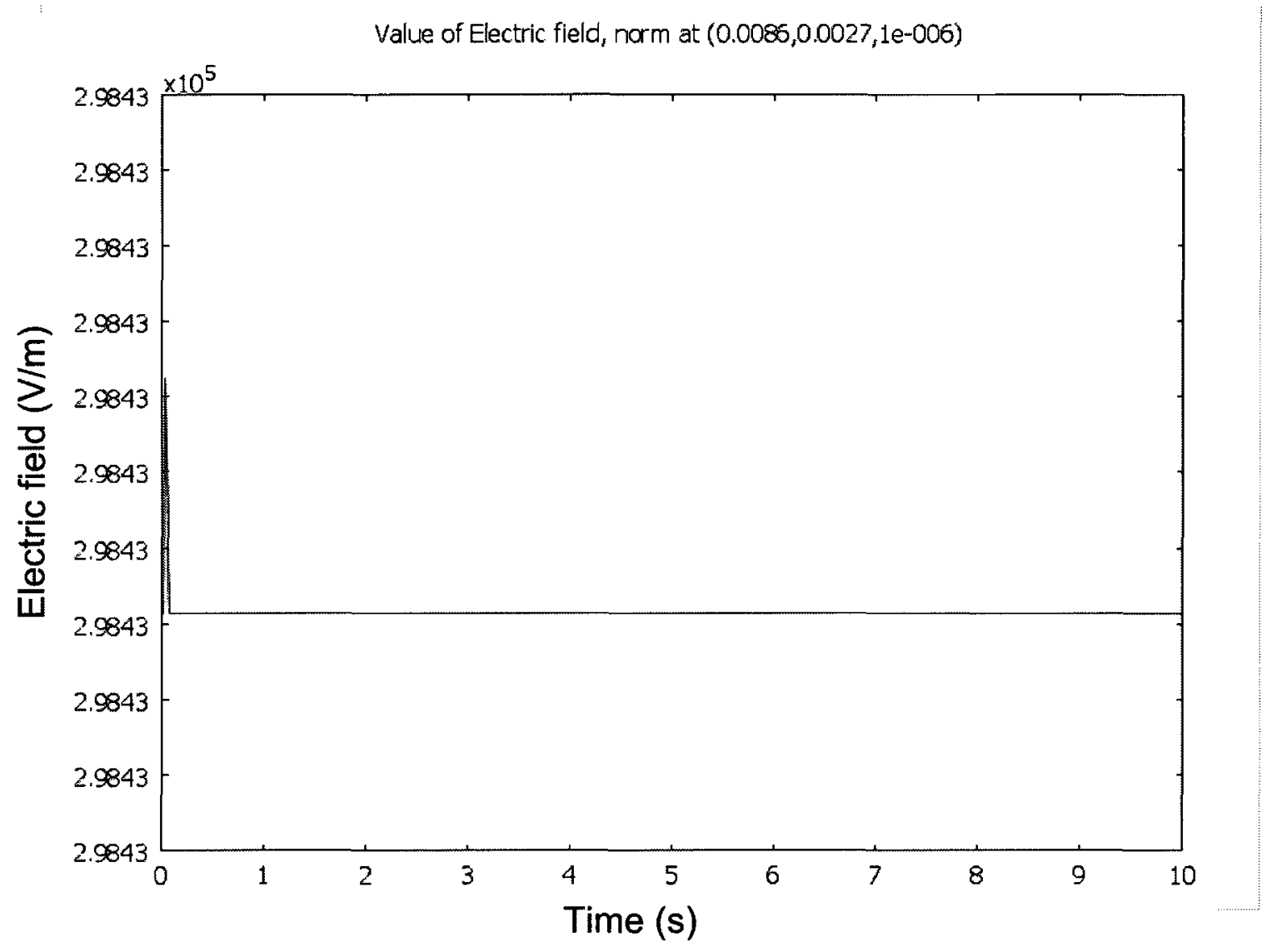

Figure 4.16 Transient development of the electric field inside the microchannel at a point $1 \mu \mathrm{m}$ above the microchannel bottom surface of the lysis chamber. The excitation was applied at time $0 \mathrm{~s}$ and continued for $10 \mathrm{~s}$. 
interest resulted from this research.

\subsubsection{Heat source}

Any conductive path, solid or liquid, results Joule heating when potential is applied due to the resistance of the path on current flow. The upper bound of such heat can be obtained by assuming that all power applied to the micro-device has been dissipated as heat. Hence, the generated heat (Q) can be determined by,

$$
Q=V I=I^{2} \times R=V^{2} / R
$$

where, $\mathrm{V}$ is the applied potential, $\mathrm{I}$ is the current flow and $\mathrm{R}$ is the resistance of the path.

For thermal simulation of microfluidic devices, FEM tools has been successfully used to model devices with simulation results closely matched by experimental results. The thermal distribution defined by second order systems coupled with proper material property and boundary conditions renders FEM tools as the preferred modelling method. A typical temperature rise due Joule heating in buffers are previously reported to be limited to 1 to $10^{\circ} \mathrm{C}$, as shown though FEM simulations of microchannel [103].

\subsubsection{Modelling of the device}

Model of the final device shown in Figure 4.12 that was created using the modeler for electrical simulation was employed for thermal simulation too. The simulation physics was changed to general heat transfer that models conductive and convective heat transfer of materials, with no radiative heat dissipation. The only regions of the model that generates heat in the device were the five microchannels in the lysis chamber as current flow through this layer that are in contact with the integrated interdigitated electrodes applying excitation potential to the micro-device. The two 
side channels, that connect the five microchannels of the lysis chamber does not contain integrated interdigitated electrodes, hence does not produce heat.

The maximum energy was dissipated in the device when the maximum voltage was applied for the maximum duration of excitation. In our experiments, the maximum voltage applied to the final device was $20 \mathrm{~V}$ and the maximum pulse duration was $5 \mathrm{~s}$. The power delivered to the device can be determined from the reading of the oscilloscope that reads $20 \mathrm{~V}$ at open circuit and $19 \mathrm{~V}$ (average) with load (microdevice). As the internal resistance of the power supply was determined to be $50 \mathrm{ohms}$. The resultant average current flow was $20 \mathrm{~mA}$. Thus the average power consumption was $0.4 \pm 0.1 \mathrm{~W}$. Hence, the maximum power consumption was $0.5 \mathrm{~W}$ that was chosen as heat source in simulations to determined the upper limit of temperature rise.

As there are five microchannels for lysis and the total power consumption is $0.5 \mathrm{~W}$, it is reasonable to assume that each microchannel consumes $0.1 \mathrm{~W}$ of power. For upper bound, assuming all applied power is converted to heat, the volumetric heat generation can be calculated by dividing the power with the volume of that microchannel. From geometric configuration of the device, the volume of each microchannel within the lysis chamber is $2 \times 10^{-10} \mathrm{~m}^{3}$. As pulses were applied for a short period of time and then withdrawn, hence transient simulations were performed that would indicate the maximum temperature with corresponding pulse widths. In addition, a steady state temperature simulation was performed to demonstrate the maximum temperature rise with continued excitation. Furthermore, a multi-physics simulation was performed with Joule heating due to electric current flow coupled with heat transfer to determine temperature distribution along the microchannel during the excitation of the device.

For thermal simulation, we have used the same Comsol Multiphysics FEM tool. This tool has a built-in modeler, meshing and solver engine for various types of physical response. For thermal simulation, general heat transfer physics model was chosen. Transient analysis were performed for $10 \mathrm{~s}$ excitation unless otherwise mentioned. Transient analysis were solved using built-in Generalized Minimal Residual 
(GMRES) solver. The relative error tolerance for convergence was 0.01 and the absolute tolerance was 0.001 . The obtained simulation results serve as a conservative estimate of the temperature that was generated right after an electric pulse excitation.

\subsubsection{Settings for sub-domains and boundary conditions}

The subdomain materials were chosen from built-in material database: substrate was glass, the microchannels and loading ports were water (liquid) and electrodes were platinum. All sub-domain temperatures were initialized at $300 \mathrm{~K}$ (assuming a room temperature of $26.8^{\circ} \mathrm{C}$ ). All internal boundaries were set to thermal conductivity. All external surface boundary of the device was set to convective heat flux dissipation with the value of the coefficient of heat transfer (h) equal to $20 \mathrm{~W} / \mathrm{m}^{2} \mathrm{~K}$, and temperature at infinite equal to $298 \mathrm{~K}$. This heat flux setting represents natural flow air convection heat dissipation [132]. Such heat flux boundary simulation provides good estimate with reduced computational complexity [84]. The range of heat transfer coefficient (h) is typically between 4.4 to $32.2 \mathrm{~W} / \mathrm{m}^{2} \mathrm{~K}$ corresponding to stationary air cooling to forced air cooling, respectively [132-134]. Calculations with our device dimensions for air flow rate of $1 \mathrm{~m} / \mathrm{s}$ resulted a heat transfer coefficient of $22.4 \mathrm{~W} / \mathrm{m}^{2} \mathrm{~K}$. To observe the effect of the coefficient of heat transfer (h) (i.e. air flow rate) on the rise of temperature of the device, we have also simulated cases with $\mathrm{h}$ set to 5,10 , and $20 \mathrm{~W} / \mathrm{m}^{2} \mathrm{~K}$.

\subsubsection{Meshing and simulation of the model}

The model was meshed using the Comsol predefined "extremely fine" parameters. The maximum growth rate was 1.3 , resulting 2258159 tetrahedron elements with 385863 mesh points and 3045076 degrees of freedom. The meshed structure is shown in Figure 4.17.

The transient thermal simulation for $5 \mathrm{~s}$ resulted a maximum temperature rise of $1.3 \mathrm{~K}$ of the fluid inside the microchannels of the lysis chamber and the temperature 


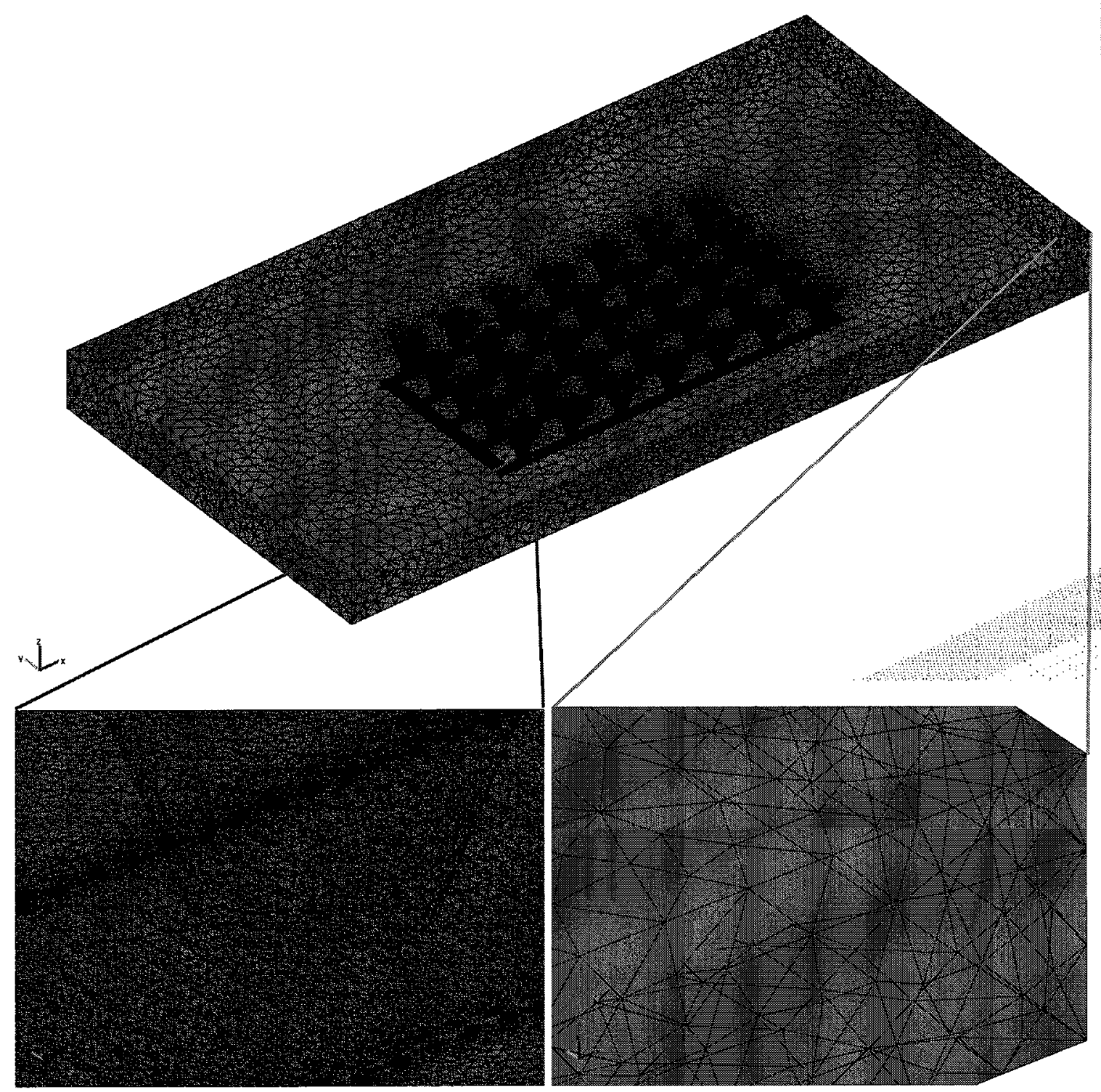

Figure 4.17 Meshed structure of the micro-device for thermal simulation with enlarged view of two important regions around the microchannel and the outer boundary. 
reached $2.2 \mathrm{~K}$ after $10 \mathrm{~s}$ of excitation (Figure 4.18 ). The maximum temperate was observed at the middle of the microchannel under the loading ports. This demonstrates convection of water with higher temperature towards the opening of the loading port. Temperatures along the surface boundaries were between 300.2 to $300.27 \mathrm{~K}$. A transient plot of temperature rise at three points inside a lysis channel $(15 \mathrm{~mm}$ along $\mathrm{x}$-axis, $2.7 \mathrm{~mm}$ along $\mathrm{y}$-axis) directly underneath a loading port is given in Figure 4.19 for excitation for $10 \mathrm{~s}$. The maximum rise of temperature was observed at the top of the microchannel $(2.25 \mathrm{~K})$, followed by the middle point of the microchannel $(2.2 \mathrm{~K})$. The rate of temperature rise can be approximated to $0.18 \mathrm{~K} / \mathrm{s}$. The temperature at the bottom of the microchannel was lower where the sample cells were expected to settle. In our experiments, the maximum pulse duration was $5 \mathrm{~s}$. The temperature increase obtained from these simulations showed small rise of temperate to affect biological elements. These results obtained with our simulation was in agreement with similar studies reported for other microfluidic devices [84]. As the excitation in our experiments were ceased after $5 \mathrm{~s}$ (maximum), these simulations provided us with the upper bound of temperatures, as the temperature would decrease after the excitation was ceased.

Note that in Figure 4.19, initial temperature rise at the middle and top of the microchannel is high, then the rate decreases to a constant value for the remaining time of simulation. This is due to the fact that in this simulation, the microchannel is the source of heat generation. Initially, heat is generated and only conductive heat transfer takes place, resulting a rapid rise of temperature. Soon the convective heat transfer through the buffer fluid inside microchannel results in a faster heat dissipation, reducing the rate of temperate increase.

For completeness of thermal simulation, we have investigated the temperature dependance on heat transfer coefficient $(h)$. As stated before, the value of $h$ varies the the convective flow of surrounding air. For example, typically for still air, $\mathrm{h}$ is $4.4 \mathrm{~W} / \mathrm{m}^{2} \mathrm{~K}$ and for natural flow of air, $\mathrm{h}$ increases up to $32.2 \mathrm{~W} / \mathrm{m}^{2} \mathrm{~K}$ depending on the flow rate $[132,133]$. As shown previously, that the expected value of $\mathrm{h}$ is 22.4 


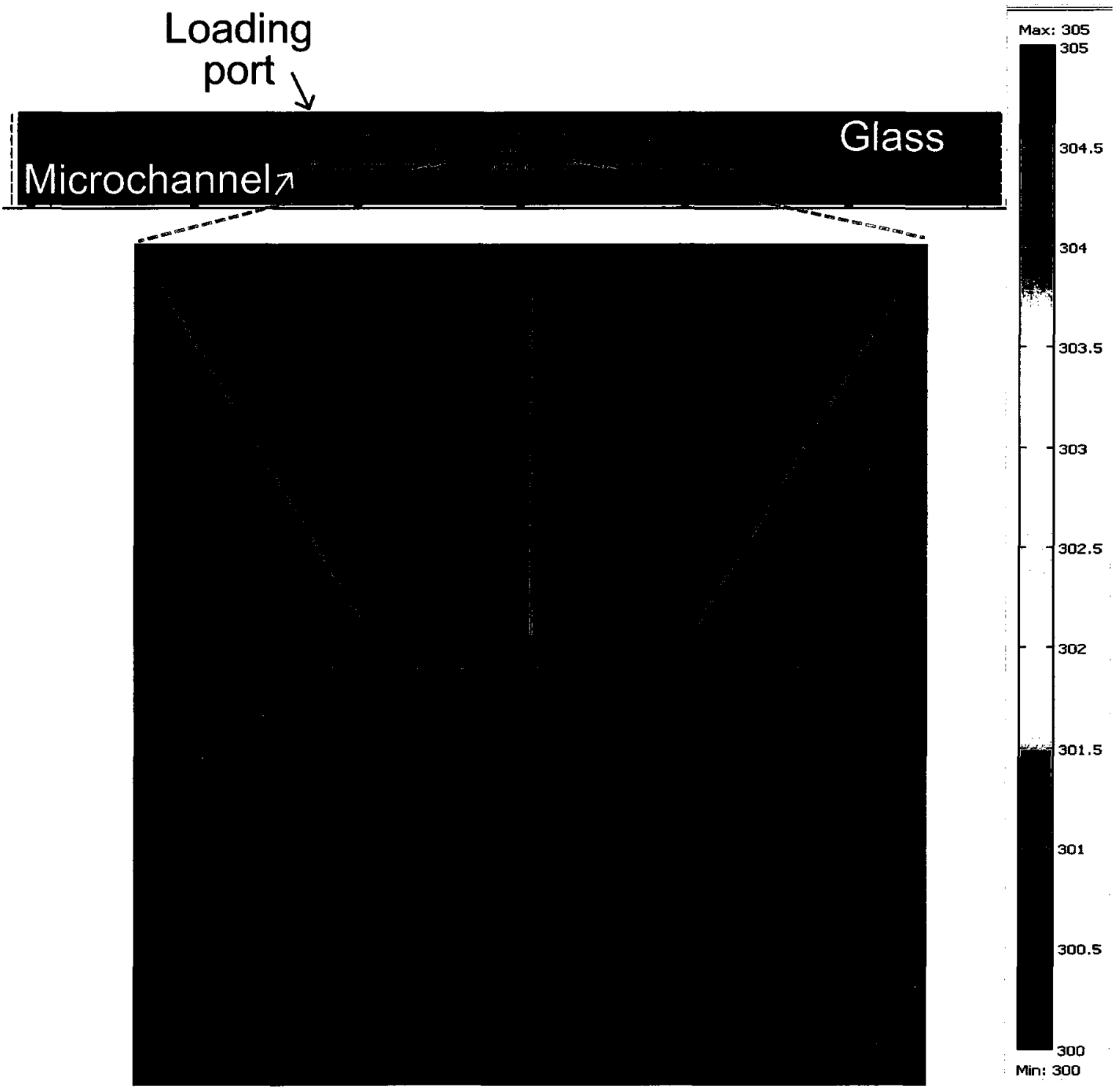

Figure 4.18 Temperature of the micro-device visualized along a xz-plane (slice plot) of the channel in the cell lysis chamber after and excitation for $10 \mathrm{~s}$. A section of the channel is enlarged to depict the corresponding temperature distribution along the height of the microchannel and the loading port. 


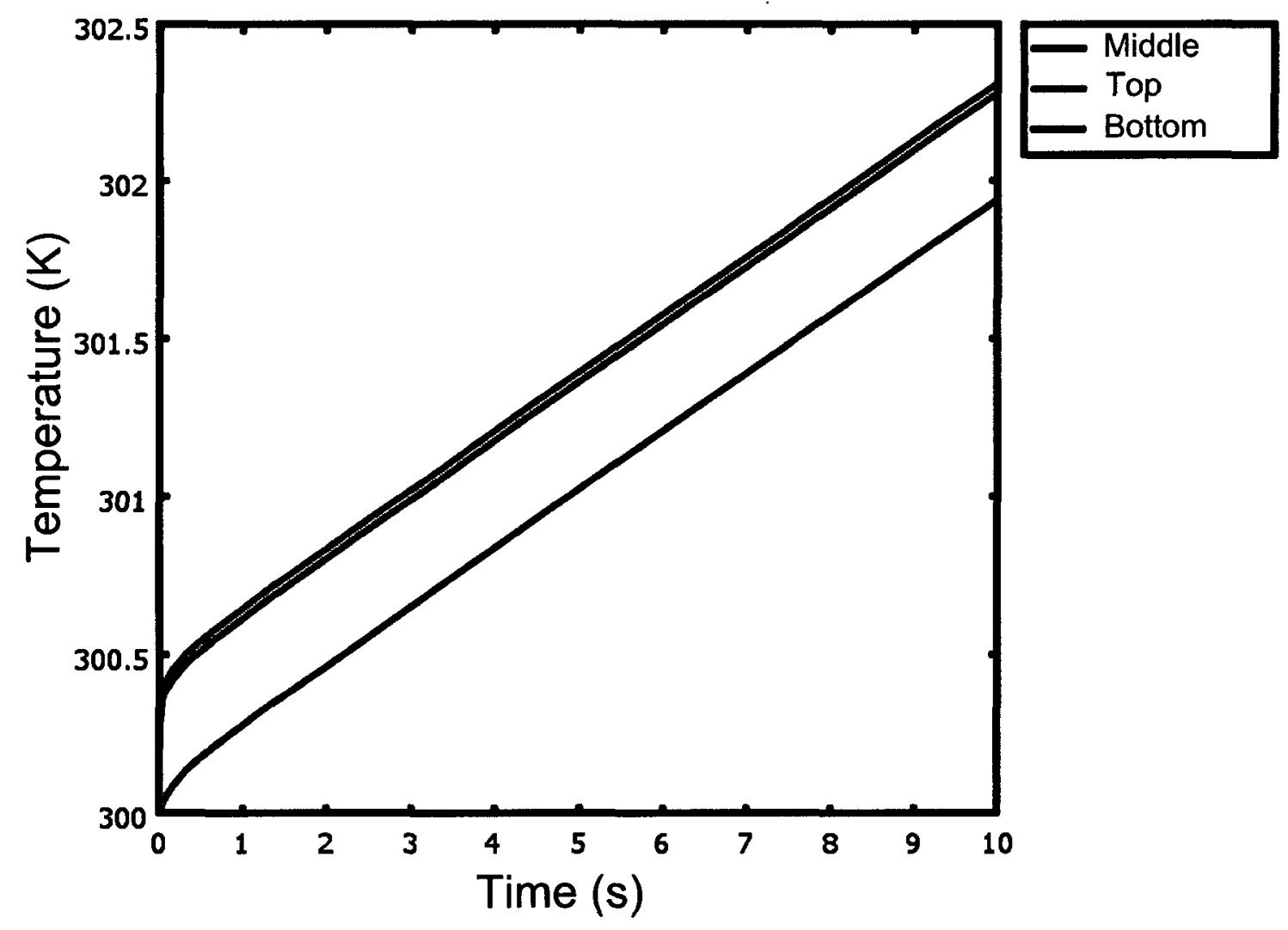

Figure 4.19 Simulation results of temperature rise inside the microchannel for lysis due to pulse excitation of $20 \mathrm{~V}$ for $10 \mathrm{~s}$. Temperature at three points were evaluated: (i) at middle of the microchannel $20 \mu \mathrm{m}$ above the bottom surface of the channel, (ii) at the top of the microchannel $40 \mu \mathrm{m}$ above the bottom surface of the channel, and (iii) at the bottom surface of the microchannel. All three points were directly underneath the middle of a loading port where the maximum temperature variation was observed. 
$\mathrm{W} / \mathrm{m}^{2} \mathrm{~K}$, and temperature would be less for higher value of $\mathrm{h}$ resulting increased heat dissipation, we performed transient simulations over $10 \mathrm{~s}$ period for $\mathrm{h}$ values of 5 and $10 \mathrm{~W} / \mathrm{m}^{2} \mathrm{~K}$, and compared that with the result of $\mathrm{h}=20 \mathrm{~W} / \mathrm{m}^{2} \mathrm{~K}$. The results are plotted in Figure 4.20. The observed temperature rise were within $2.9 \mathrm{~K}$ for all cases for up to $10 \mathrm{~s}$ of excitation. As expected, a lower value of $\mathrm{h}$ would result a higher temperature, but the difference was not substantial to cause any effect on biological elements. For instance, for excitation of $5 \mathrm{~s}$ duration, the rise of temperature were $1.7^{\circ} \mathrm{C}, 1.45^{\circ} \mathrm{C}$, and $1.3^{\circ} \mathrm{C}$ for $\mathrm{h}$ set to 5,10 and $20 \mathrm{~W} / \mathrm{m}^{2} \mathrm{~K}$, respectively. To determine the maximum limit of temperature rise based on $\mathrm{h}$, a simulation assuming $\mathrm{h}=0$ resulted a temperature rise of $1.98^{\circ} \mathrm{C}$ after $5 \mathrm{~s}$ and $3.43^{\circ} \mathrm{C}$ after $10 \mathrm{~s}$. The maximum temperature rise at boundary of the device was $0.86^{\circ} \mathrm{C}$ after $10 \mathrm{~s}$ with such excitation. From these simulations, it was noted that the maximum temperature rise would not effect biological elements as they are usually subjected to a higher temperature variation in nature.

To determine mesh dependency on these thermal simulations, Comsol predefined "fine" parameters was chosen where the maximum growth rate was decreased to 1.45 resulting about 1 degree of lower number of elements (about half the number of elements in each axis). This resulted 374817 elements with 64642 mesh points and 507471 degrees of freedom. Simulation with this new mesh size resulted a temperature rise of $1.36^{\circ} \mathrm{C}$ after $5 \mathrm{~s}$ and $2.19^{\circ} \mathrm{C}$ after $10 \mathrm{~s}$ with $\mathrm{h}$ parameter set to $20 \mathrm{~W} / \mathrm{m}^{2} \mathrm{~K}$. The difference in results with about one degree of lower mesh were $0.05^{\circ} \mathrm{C}$ at $5 \mathrm{~s}$ and $0.07^{\circ} \mathrm{C}$ at $10 \mathrm{~s}$. These errors were within less than $4 \%$ indicating acceptable mesh invariance of these temperature simulations.

We have further investigated the steady state temperature that would result if the excitation was continued. The resultant temperature distribution is given in Figure 4.21. The maximum temperature rise was $22.98^{\circ} \mathrm{C}$ with similar distribution of temperature as observed for transient analysis. With the rate of temperature increase obtained from the transient analysis, the approximate time to reach this steady state would be 2.12 minutes of continued excitation. Thus such temperature 


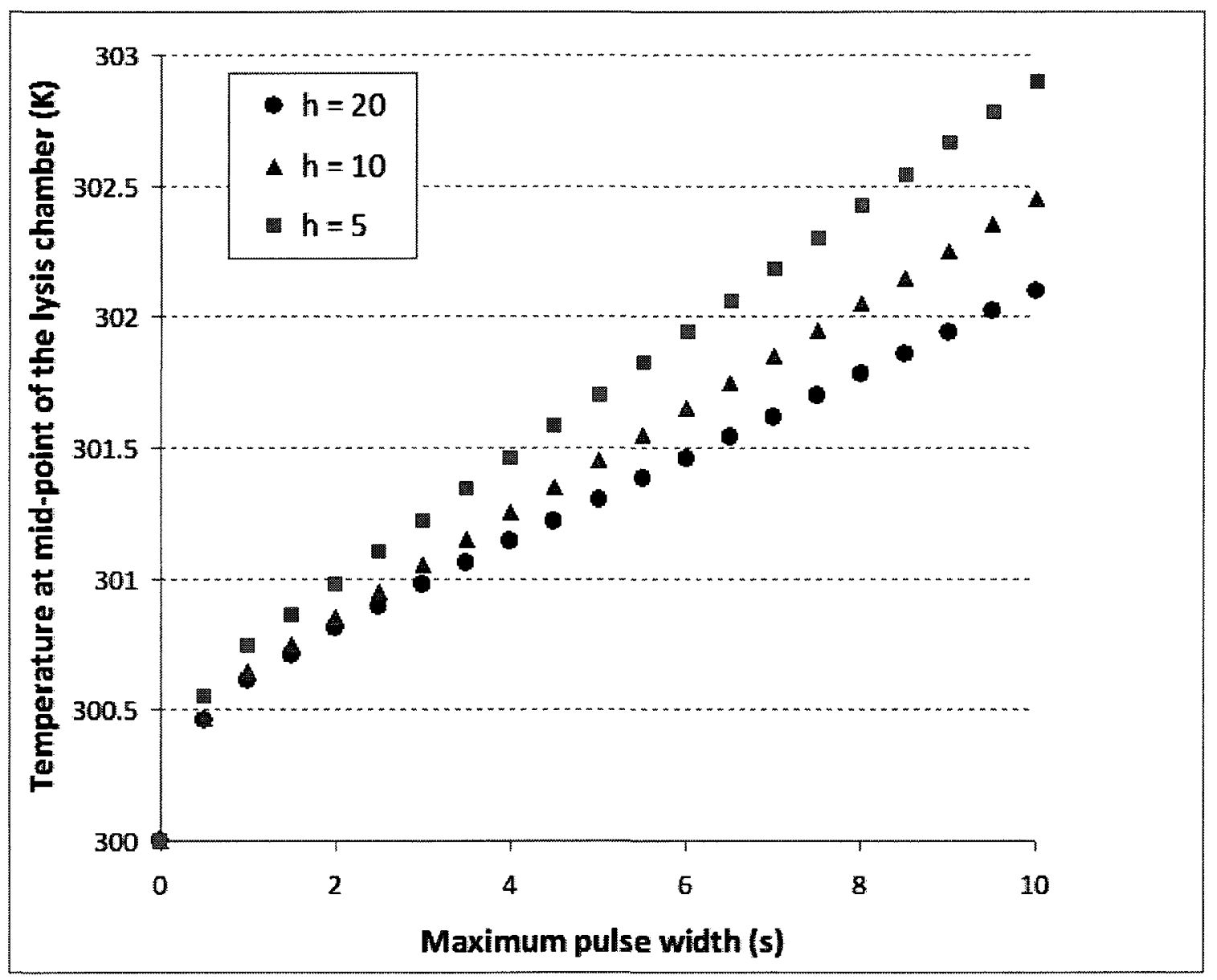

Figure 4.20 A plot showing transient increase of temperature for the values of heat transfer coefficient $(\mathrm{h})$ set to 5,10 and $20 \mathrm{~W} / \mathrm{m}^{2} \mathrm{~K}$, as an electric pulse excitation of $20 \mathrm{~V}$ continues for $10 \mathrm{~s}$. The initial temperature was of all sub-domain was assumed to be at room temperature of $300 \mathrm{~K}$. 
was not expected to occur in any of our experiments, however this result serves as an absolute upper bound of temperature increase within the micro-device. It is worth to note that this temperature rise was less than the temperature rise required for thermal lysis ( 70 to $80^{\circ} \mathrm{C}$ [90]), indicating that such device would not be suitable for thermal lysis of cells.

Furthermore, we have investigated the spatial distribution of temperature due to the current flow through the electrodes and microchannels resulting Joule heating. This was modelled with a multi-physics simulation environment of Comsol with Joule heat model coupled with general heat transfer model. The model of the a section of the device is shown in Figure 4.22. The model was meshed with very fine resolution of surfaces where electrodes and channels intersected (Figure 4.23). This high resolution mesh of these surface were needed as there would be a large physical parameter change across the tetrahedrons at this surfaces. The average number of nodes between electrode gaps and on top of electrodes were 5, estimated from zooming to the corresponding mesh area.

The transient simulation result showed a temperature rise of $3.43^{\circ} \mathrm{C}$ after $10 \mathrm{~s}$ (Figure 4.24). The simulation result showed temperature gradients inside and outside the microchannel. The higher temperature value compared to previous transient analysis results is attributed to non-uniform distribution of heat within the microchannel as imposed by the physics in this simulation. The higher temperature region was located at the middle of the microchannel, that can be attributed to the thermal conductivity properties of various materials. The interface of electrodes and channel did not exhibit the maximum temperature as the higher temperature conductivity of the metal layer would conduct heat quicker to a larger surface compared to convective heat dissipation inside the microchannel.

Thus it was demonstrated exhaustively through these thermal simulations that the upper bound of temperature rise of the device due to the excitation applied to this device was less than $4^{\circ} \mathrm{C}$ for a $20 \mathrm{~V}$ pulse of $10 \mathrm{~s}$ duration considering all transient simulation cases. This pulse parameter serves as the upper bound as the 


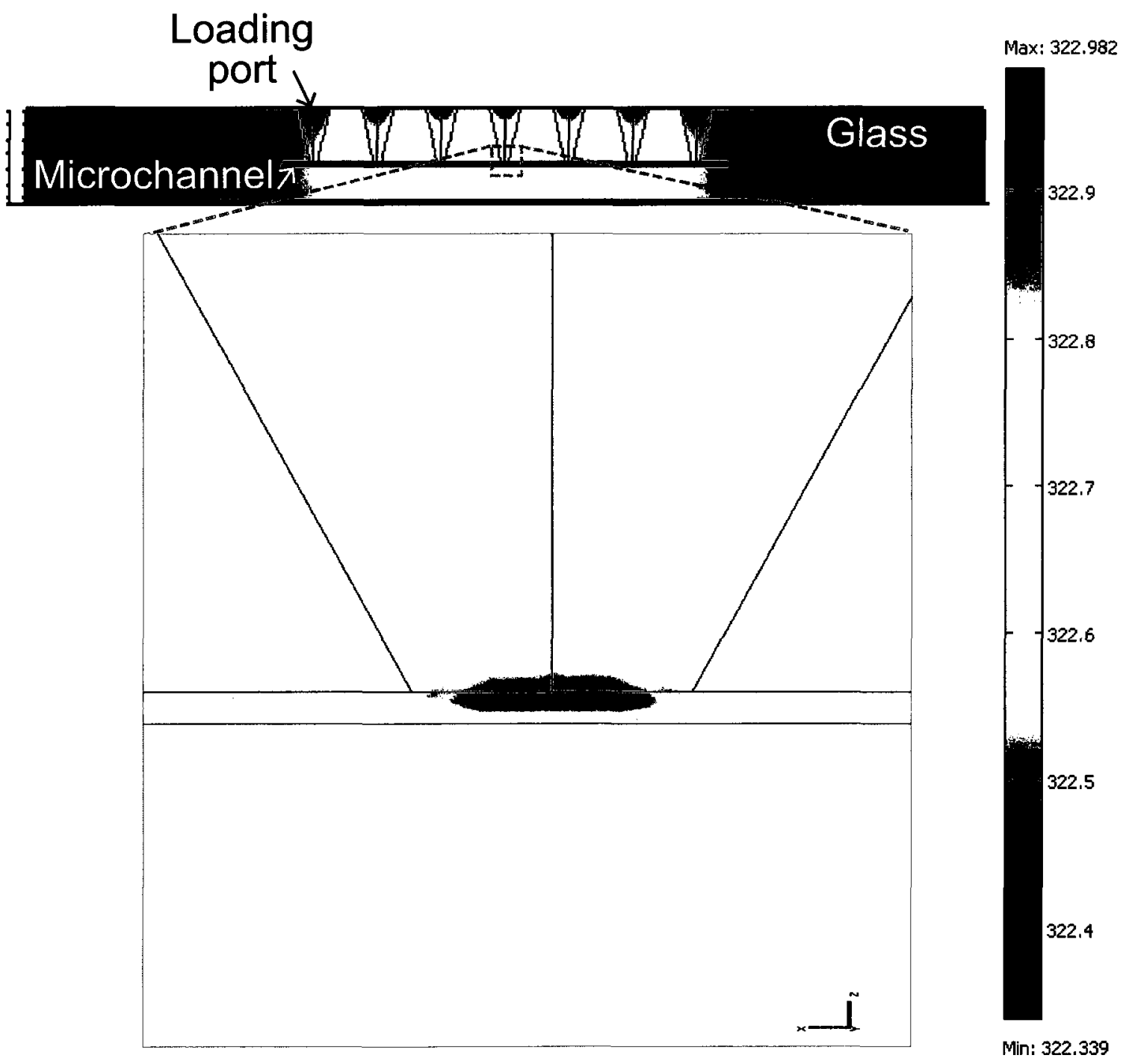

Figure 4.21 Steady state temperature simulation results showing a temperature increase up to $22.98 \mathrm{~K}$ from room temperature. 


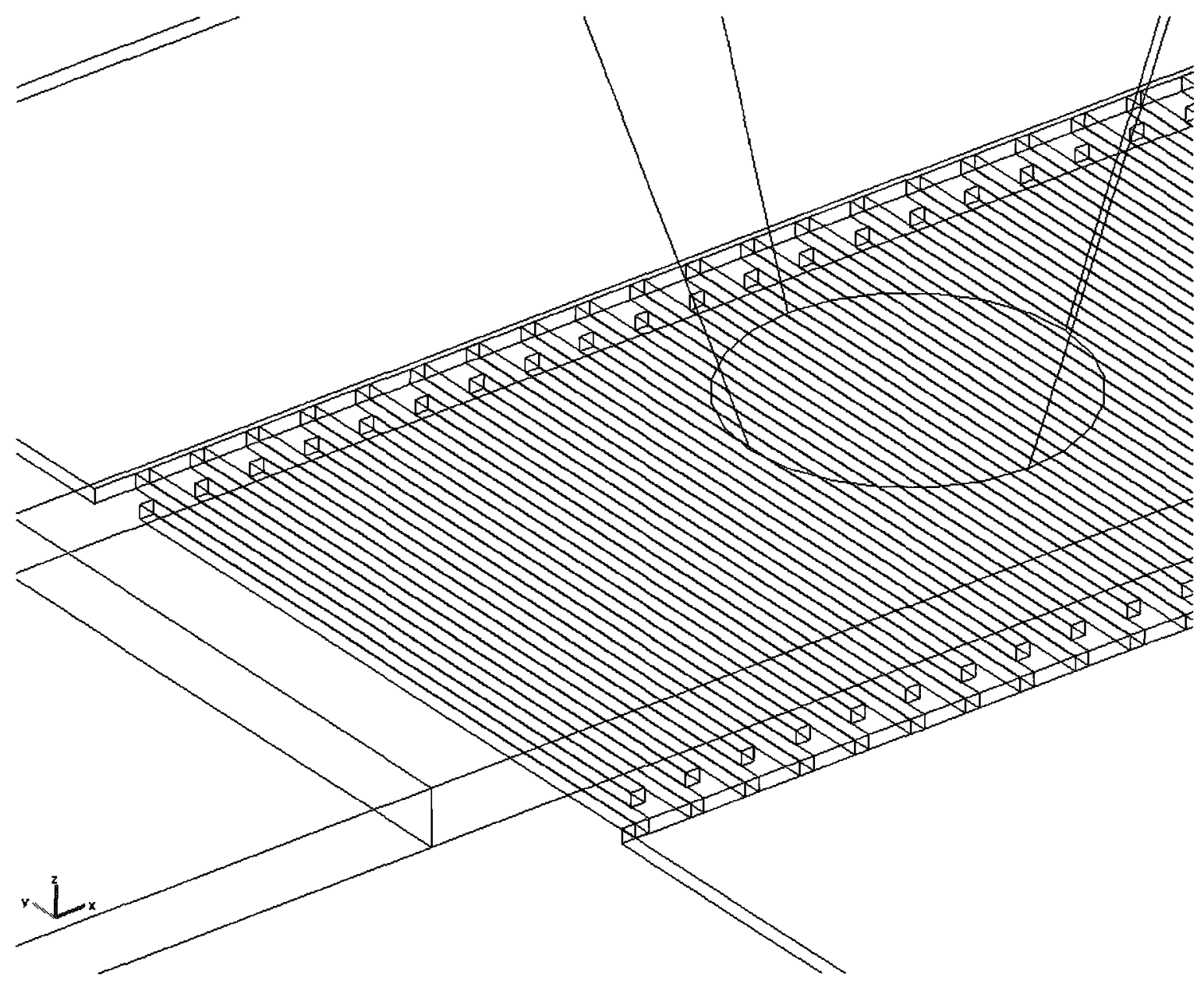

Figure 4.22 A section of the model is enlarged to show electrodemicrochannel configuration for Joule heating coupled heat transfer multiphysics analysis. 


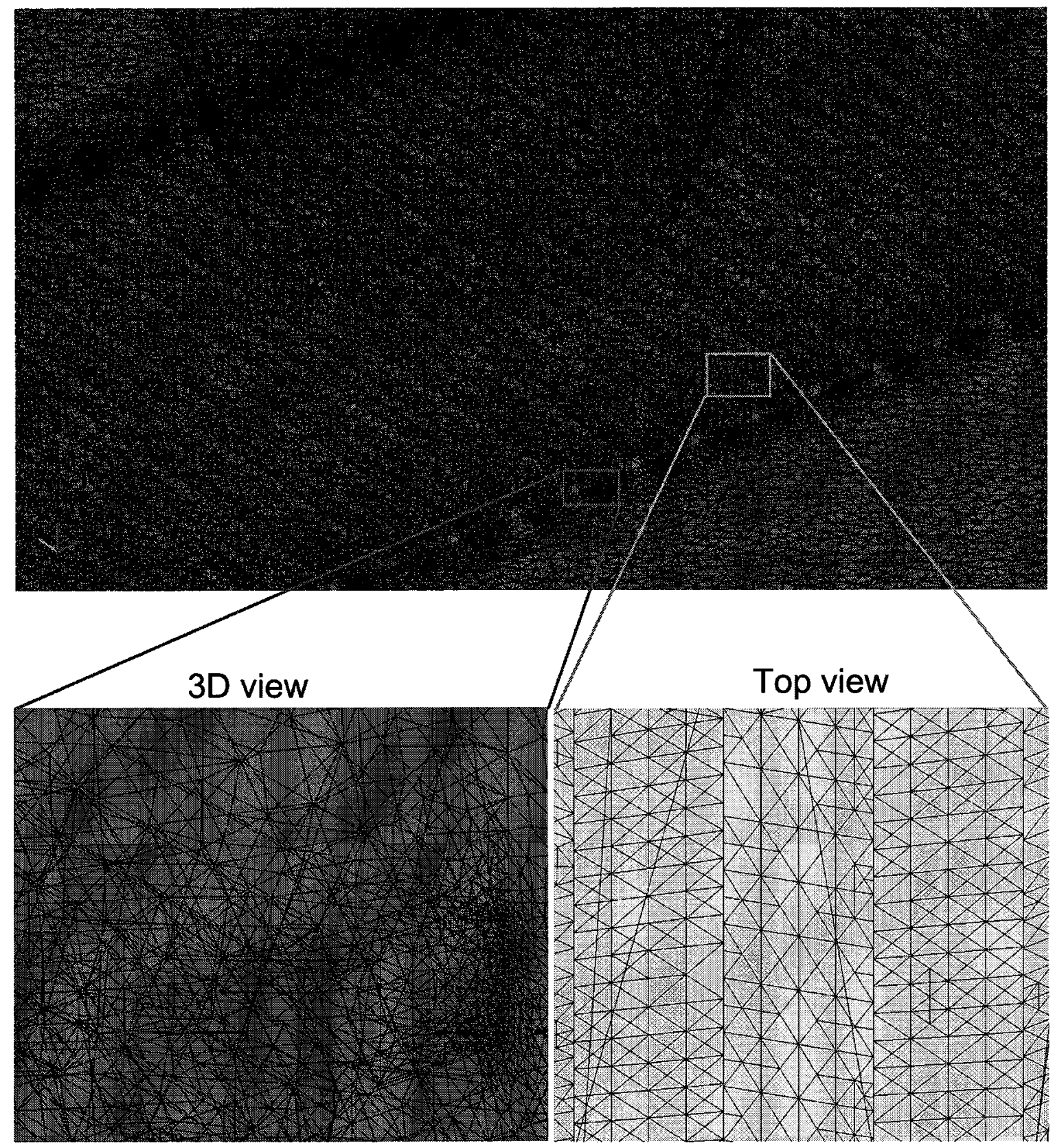

Figure 4.23 Detailed view of mesh around the electrodes for the multiphysics FEM simulations. In the insets at the bottom, meshes of two sections of importance are shown. 


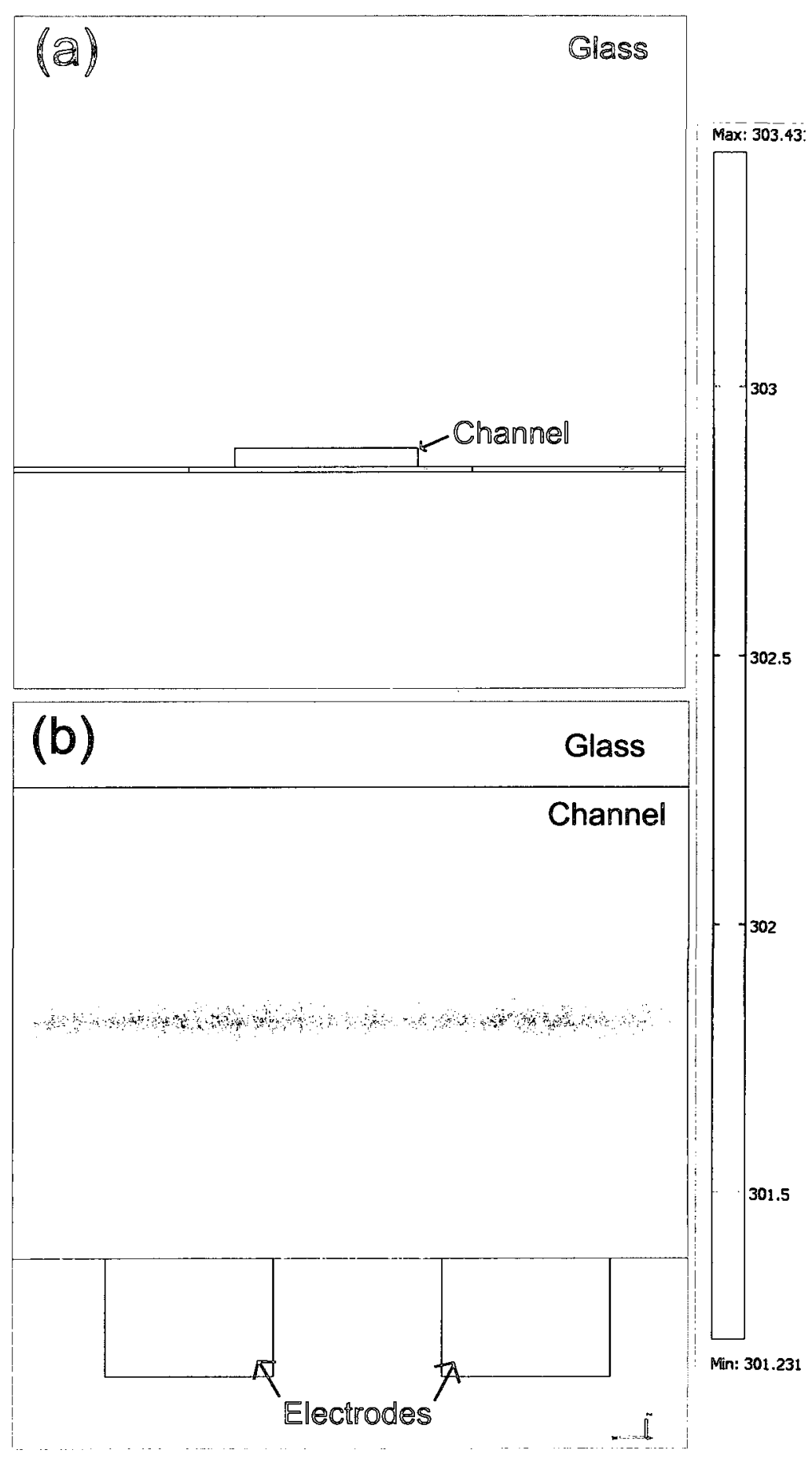

Figure 4.24 Multi-physics simulation of Joule heating coupled with heat transfer depicting the temperature rise for a $10 \mathrm{~s}$ excitation with $20 \mathrm{~V}$ of applied voltage through the integrated electrodes. (a) Cross-section view of the microchannel at the middle of two electrodes. (b) Axial-section view of the microchannel showing temperature distribution inside the microchannel. 
heat produced was higher than all experimental cases for EL or EP. As biological cells are commonly exposed to a larger range of temperature variation in nature (typically about $10^{\circ} \mathrm{C}$ ), this temperature rise due to the device excitation is not expected to cause any significant change of cell morphology. In fact, for thermal lysis, the required temperature is 70 to $80^{\circ} \mathrm{C}$ [90]. In addition, during Polymerase Chain Reaction (PCR) amplification in downstream DNA processing, a temperature cycle of up to $95^{\circ} \mathrm{C}$ are routinely applied on DNA in the denaturing phase of DNA strands without any damage to DNA. 


\section{Chapter 5}

\section{Experimental Results}

This chapter discusses on the experimental results obtained with the developed devices. Experimental setup, equipment and chemicals, experimental procedure, as well as analysis of results for each devices have been described separately.

\subsection{The Initial Device}

The initial device was developed using Protolyne Microfluidic fabrication process. The fabricated device was tested in the labs of Cardiovascular Device Division of Ottawa Heart Institute, Ottawa.

\subsubsection{Chemical reagents}

Dulbecco's Phosphate Buffered Saline (D-PBS) fluid from Invitrogen corporation, Carlsbad, CA, USA, was used to suspend debris collected from the fingerprint samples and to fill the microchannels and the reservoirs. Harris Hematoxylin from Fisher Scientific, Pittsburgh, PA, USA, was used to stain nucleic acid of samples. Alexa Fluor 488 annexin V from Molecular Probes Inc, OR, USA, was used as the fluorescence dye for cell marking with fluorescence microscope. 


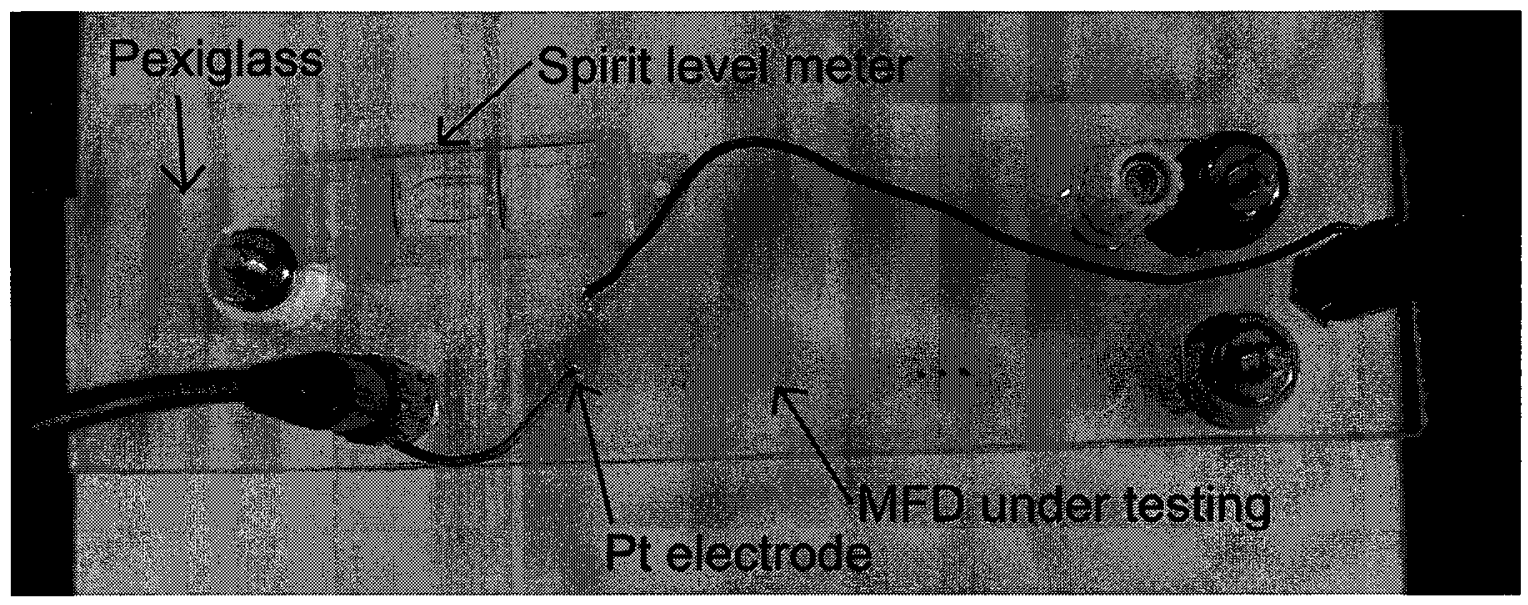

Figure 5.1 A photograph of the custom-made platform for holding the MFD during the experiments.

\subsubsection{Equipment and setup}

A high voltage power supply unit of model FB600 from Fisher scientific, Pittsburgh, PA, USA, was used as the source of the excitation voltage. An oscilloscope of model TDS 3012 from Tektronix Inc, USA, was used to record pulse waveforms. A multifrequency LCR meter of model HP 4274A from Agilent Technologies, USA, was used for impedance measurement. A custom-made Plexiglass-based platform was used to secure the device and electrodes in place during experiments (Figure 5.1). Experiments were conducted on the stage of an optical microscope of model CKX41 from Olympus corporation, Westmont, IL, USA, equipped with a Charge-Coupled Device (CCD) camera connected to a computer. Fluorescence images were captured by a florescence microscope of model Fluoview FV1000 from Olympus Imaging America Inc, PA, USA. Scanning Electron Microscope (SEM) photographs were taken using a digital SEM of model JSM-6400 from JEOL Canada Inc, QC, Canada.

The experimental setup is shown schematically in Figure 5.2. Channel 1 of the oscilloscope captured the waveform of the applied pulse, whereas channel 2 captured the voltage across a known resistance $\left(R_{s}\right)$. Current flow can be calculated by dividing channel 2 data by the value of $R_{s}$. 


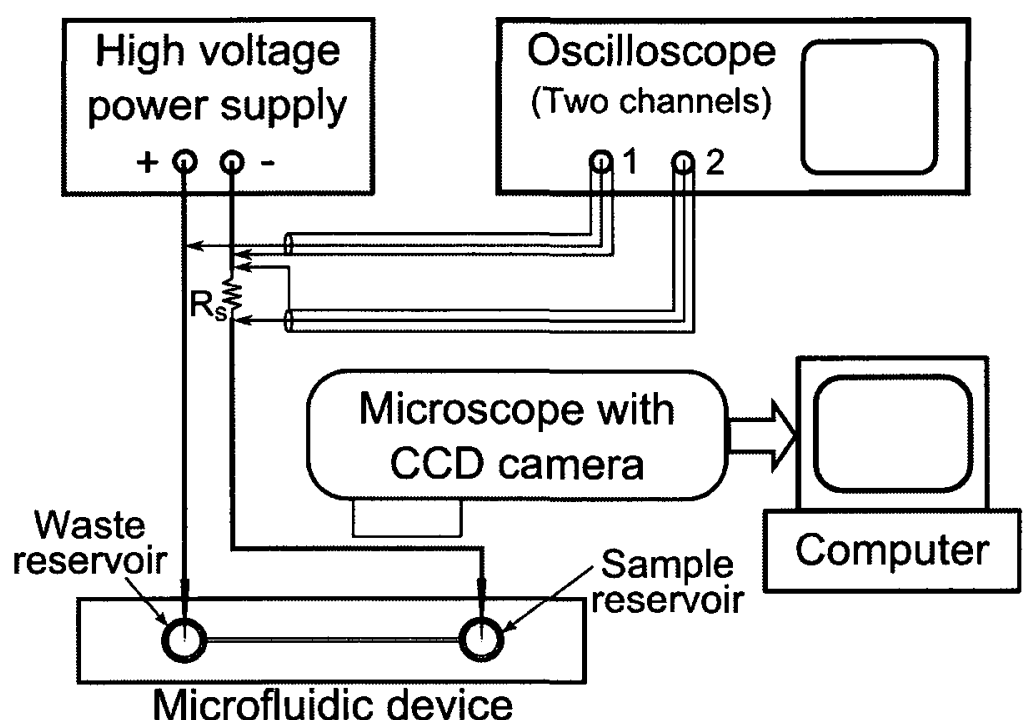

Figure 5.2 A schematic representation of the experimental setup of the MFD for the electrical lysis experiments.

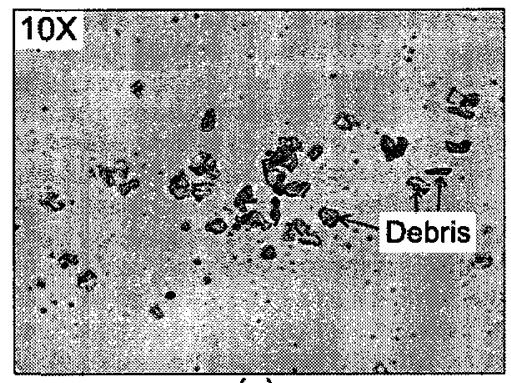

(a)

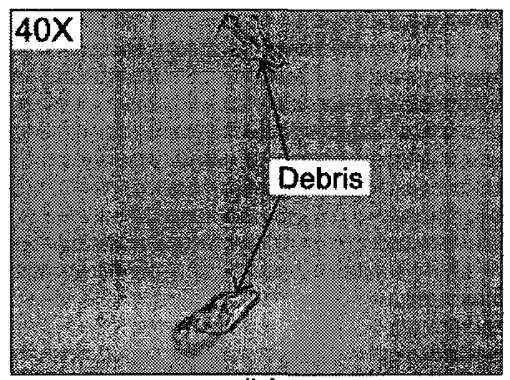

(b)

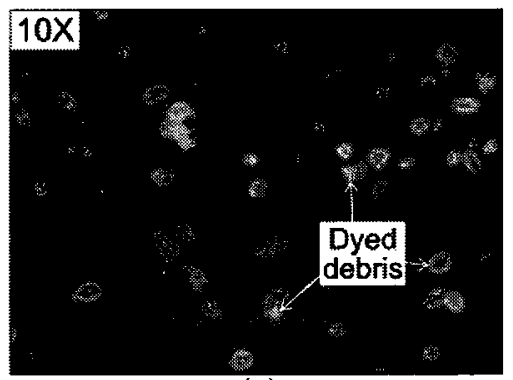

(c)

Figure 5.3 Cell debris of a fingerprint sample observed under (a) $10 \times$ and (b) $40 \times$ lens. (c) Staining using Hematoxylin dye shows presence of cells (nucleic acid) within the sample.

\subsubsection{Sample collection and preparation}

Human fingerprints on Petri dishes were used as the sources of non-invasively collected sample cells. Fingerprints compose of debris that includes shredded dead cells and cell fragments from the epidermal tissue (skin). To verify existence of cells and cell debris in fingerprint samples, a sample dish was stained with Hematoxylin, a nucleic acid stain, using a staining procedure for attached cells and observed under the optical microscope. A photograph of a sample case is shown in Figure 5.3, where cells can be identified through stained nucleic acids. This staining was only done to verify 
existence of cell debris in human fingerprint samples, and was not a part of the procedure for sample preparation or the developed method of Electrical Lysis (EL).

Each fingerprint sample was taken from a finger of an individual on a sterile Petri dish. To collect these debris from the surface of the Petri dishes, a $20 \mu \mathrm{L}$ of $1 \mathrm{M}$ D-PBS buffer fluid is applied using a micropipette. The D-PBS fluid maintains a pH of about 7.0 to keep the cells is a stable condition. The micropipette tip is rubbed on the surface of the dish to suspend the cells and debris into the fluid for about 20 seconds. This fluid is then collected back in the micropipette as the sample solution. Average number of debris on the surface for a fingerprint from a wet finger before the collection procedure was 41.5 per $\mathrm{mm}^{2}$ and after the procedure was 21 per $\mathrm{mm}^{2}$, a collection efficiency of about $50 \%$ (averaged data from 5 samples, $25 \mathrm{~mm}^{2}$ area each). Collected samples were stored in Eppendorf centrifuge tubes at the room temperature for the experiments within 24 hours.

\subsubsection{Brief description of the method}

Before every experiment, the Microfluidic Device (MFD) was washed with flowing deionized water $\left(\mathrm{D}-\mathrm{H}_{2} \mathrm{O}\right)$ and dried with blowing warm air. The MFD was secured on the custom-made platform on top of the stage of an optical microscope equipped with a CCD camera. A $4 \mu \mathrm{L}$ of the sample solution is introduced in one access hole (acting as the sample reservoir), while a $4 \mu \mathrm{L}$ of $1 \mathrm{M} \mathrm{D}-\mathrm{PBS}$ buffer fluid is introduced in an adjacent access hole (acting as the waste reservoir) that is connected with the sample reservoir through a microchannel. A platinum electrode-pair was introduced in both reservoirs and successful loading of the buffer was verified as described in Section 5.1.6. If the buffer loading was successful, the electrode-pair was connected to a high voltage power supply and an oscilloscope through a known series resistance $\left(R_{s}\right)$ as shown in Figure 5.2.

To move cells through the microchannel, a constant electric potential of 100 to 200 $\mathrm{V}$ was applied through the electrode in the waste reservoir, while the electrode in the 
sample reservoir was kept at the ground potential. This step created a small electric field (below $25 \mathrm{kV} / \mathrm{m}$ ) along the microchannel. Under the influence of this electric field generated force, known as electrophoresis (the motion of electrically charged particles under the influence of an electric field [135]), the sample cells (electronegative in nature) moved from the sample reservoir into the microchannel towards the waste reservoir.

When the cells entered the microchannel, a high voltage (about $750 \mathrm{~V}$ to $900 \mathrm{~V}$ ) was applied for a brief period of time (less than 10 seconds) through the electrode-pair to create a high electric field (about $100 \mathrm{kV} / \mathrm{m}$ or higher) to initiate EL. Sequence of photographs were taken in the phase-contrast mode using the CCD camera attached to the optical microscope. The camera parameters were tuned to take snaps every 0.5 seconds. As soon as the high voltage was turned on, the frame number being captured was noted so that snaps after application of the high voltage can be identified.

\subsubsection{Measurement of impedance of microchannels}

To determine the impedance of the microchannels, measurements were taken using an LCR meter with 1M D-PBS buffer filled microchannels for various lengths. A 8 $\mathrm{mm}$ long microchannel is analyzed in detail here as a case study. The magnitude and angle of the impedance $(Z)$ were measured using the LCR meter. Averaged data are plotted in Figure 5.4. The plots shows that, for this microchannel, the resistive component $\left(X_{R}=\Re Z\right)$ dominates up to a frequency of about $20 \mathrm{kHz}$, beyond which the reactive component $\left(X_{C}=\Im Z\right)$ becomes significant. The real part of the impedance represents resistive component, which decreases with increased frequency. The imaginary component, however increases to rising frequency. The negative values of imaginary component denotes the reactance is capacitive type.

The experimental data is conforms to the lumped equivalent circuit model consisting of a parallel combination of a resistor $(R)$ and a capacitor $(C)$. Here, the values of $\mathrm{R}$ and $\mathrm{C}$ used for simulation was $2.7 \mathrm{M} \Omega$ and $\mathrm{C}=1.4 \mathrm{pF}$, respectively. Calculation 

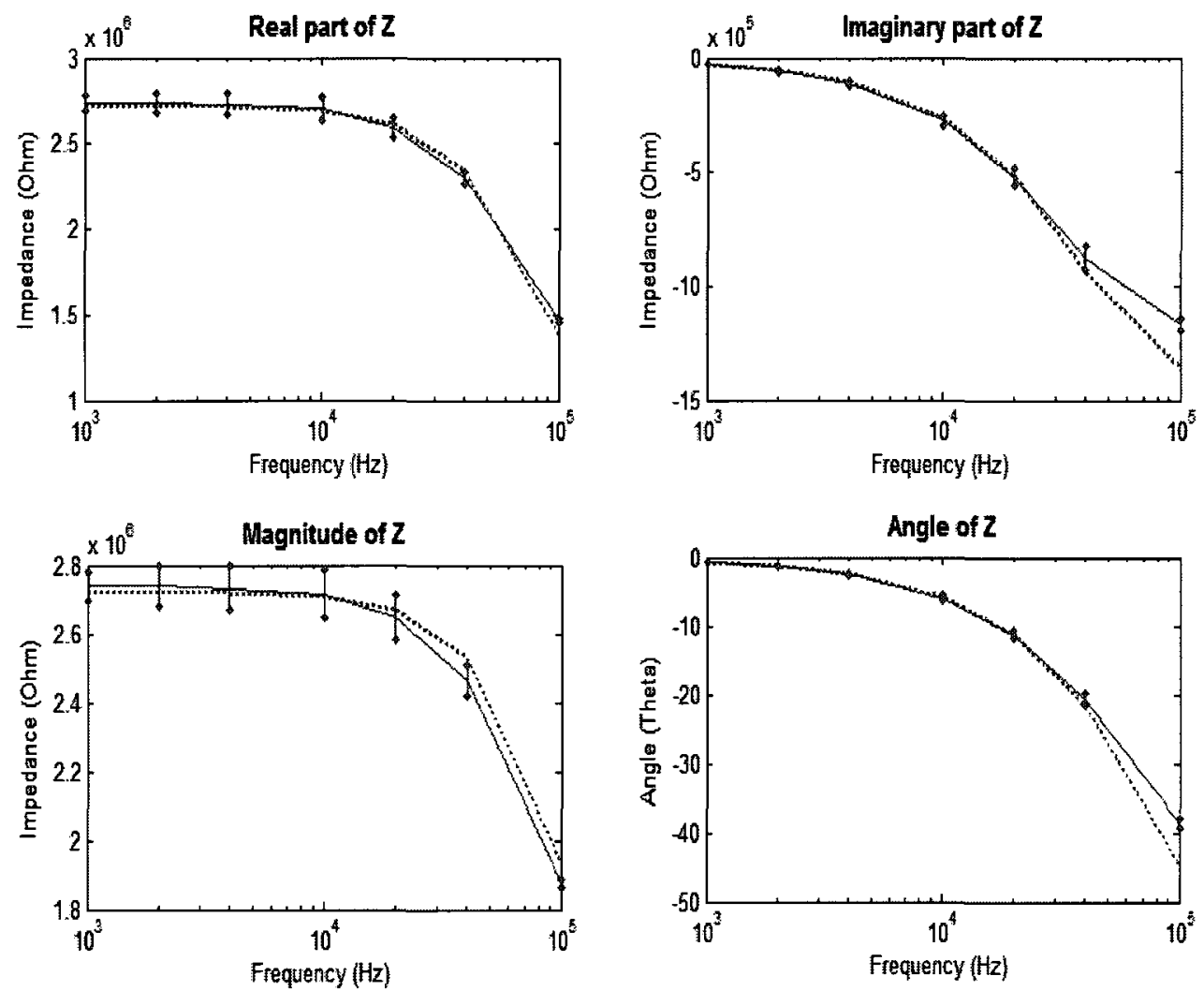

- Experimental results .... Theoretical model

Figure 5.4 Average impedance microchannels of the device is plotted for a frequency range of $1 \mathrm{kHz}$ to $100 \mathrm{kHz}$ in two sets of graphs: first set showing real and imaginary components of the impedance, while the second set depicting the magnitude and angle of the impedance. In all cases, standard deviations are shown for measurement points as vertical lines. The experimental results are matched with simulation of theoretical model with a parallel combination of a resister $(R)$ of $2.7 \mathrm{M} \Omega$ and a capacitor $(C)$ of 1.4 $\mathrm{pF}$. 
of $\mathrm{R}$ for the microchannel dimensions results $2.5 \mathrm{M} \Omega$ resistance for the microchannel, the rest can be contribution from the reservoirs with $0.1 \mathrm{M} \Omega$ resistance each. However similar calculation for capacitance with microchannel dimensions results $0.17 \mathrm{fF}$, about $10^{4}$ times less than the simulation value. The reason for such mismatch of capacitance is the large electric flux through the glass that is unaccounted for in this calculation.

\subsubsection{Loading of buffer fluids in microchannels}

When the buffer fluid was introduced in a reservoir using a micropipette, the fluid instantly flowed through the microchannel connected to the reservoir. If there was no blockage inside the microchannel, the buffer fluid reached the other end of the microchannel without any pumping action, except for the case of $55 \mathrm{~mm}$ long microchannel. It is believed that the surface tension of the buffer fluid was primarily responsible for this type of free flow. The flow rate of the buffer fluid in the microchannel was estimated to $1 \mathrm{~cm} / \mathrm{s}$.

A case study of an $7 \mathrm{~mm}$ long microchannel is presented here to determine the electrical characteristics of blocked and not-blocked microchannels. As the electrodepair was introduced in two adjacent reservoirs, connected through a blockage-free microchannel filled with the buffer fluid, a mV-range decaying electrical potential was detected (Figure 5.5). The magnitude of this potential depends on a number of factors including the number of free ions in the buffer fluid, surface area of electrodes in buffer fluid etc. The presence of a blockage (due to trapped air-bubbles) inside the microchannel resulted in a near-zero potential across the electrodes. Besides, blocked microchannels resulted in significantly higher resistances compared to microchannels without any blockage. Averaged data from experiments are presented in Figure 5.6. Averaged potential generated for not-blocked microchannels was $200 \mathrm{mV}$, whereas for blocked microchannels was $10 \mathrm{mV}$. Similarly, the average impedance measured for not-blocked microchannel was $1.75 \mathrm{M} \Omega$, whereas for blocked microchannel was 


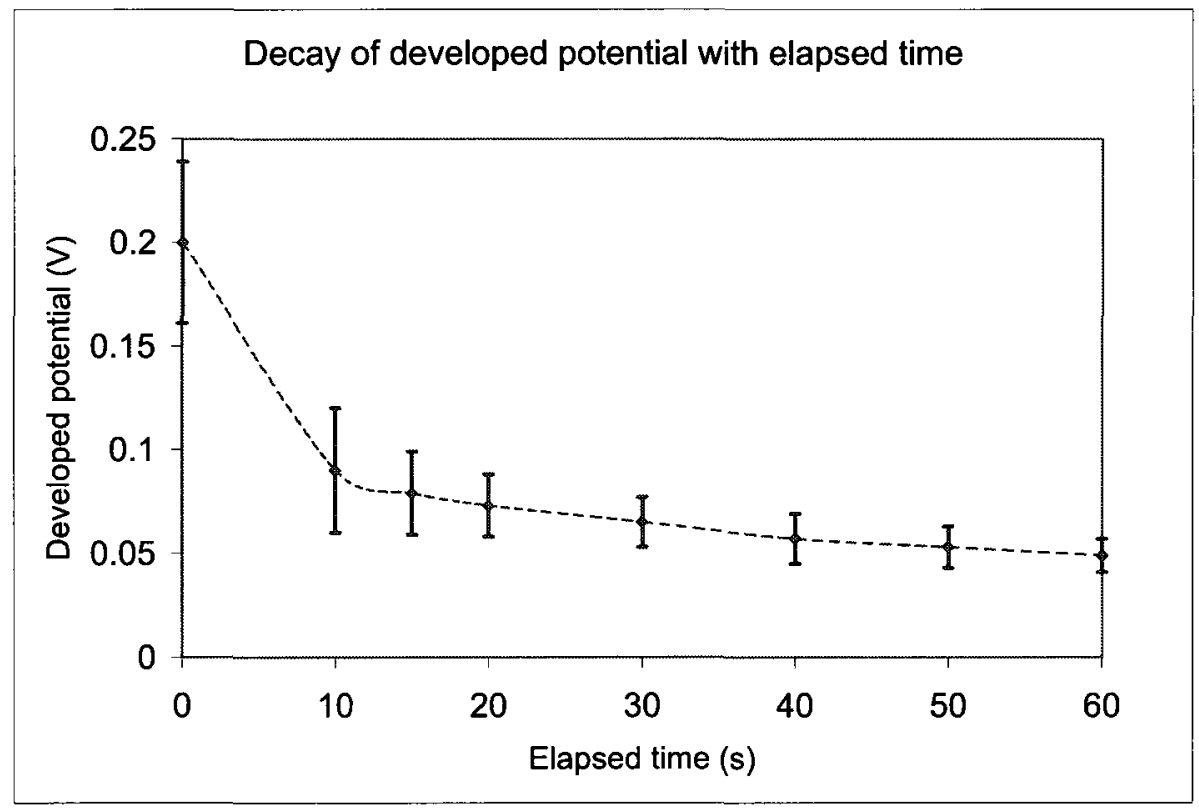

Figure 5.5 Plot showing average values of the decaying potentials developed in electrode-pairs for a $7 \mathrm{~mm}$ long microchannel. The error bars represent the standard deviations. The number of trials were 10 . 


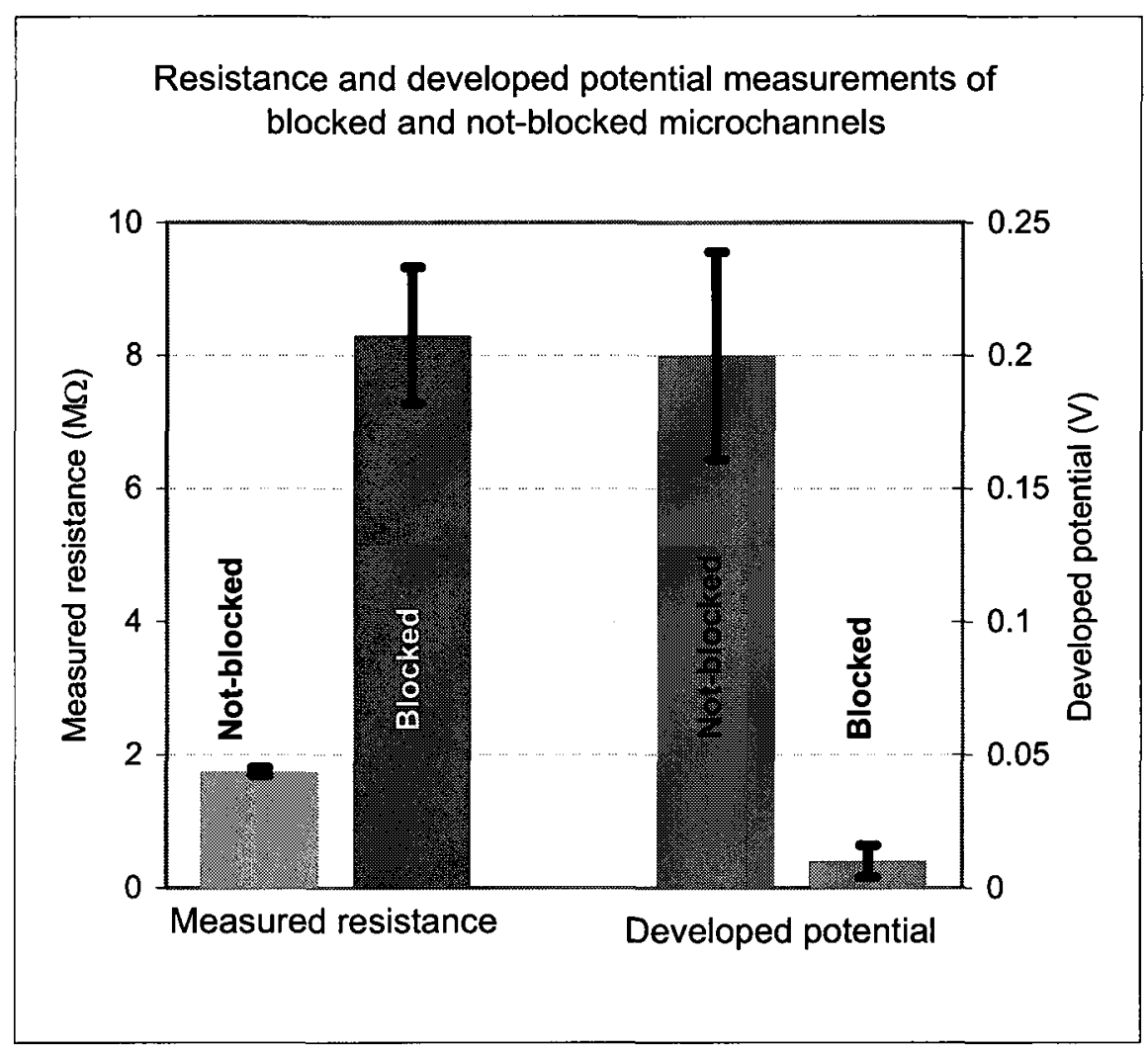

Figure 5.6 Averaged data are plotted for means (shown as bars) and standard deviations (shown as errors) for not-blocked and blocked microchannels of $7 \mathrm{~mm}$ long. The plots on the left are for measured resistances of the microchannels, and on the right are for potentials developed when electrode-pair was introduced in the reservoirs. The number of trials were 20. 

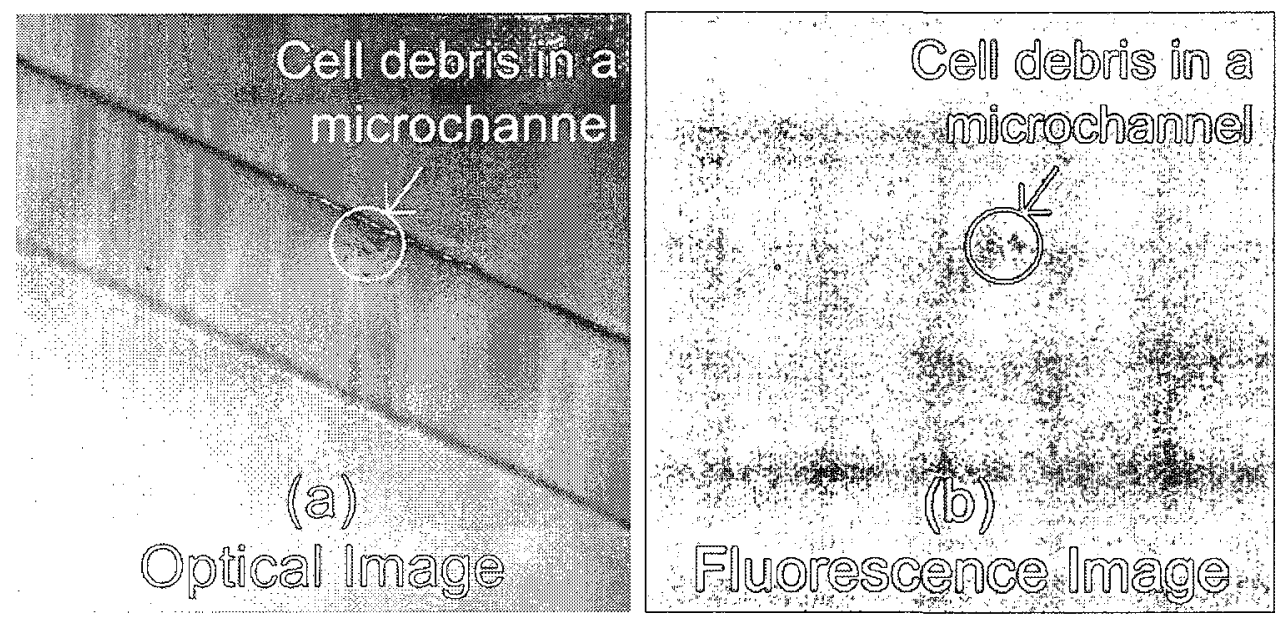

Figure 5.7 A fluorescence dyed cell collected from a fingerprint sample was positioned inside a microchannel and observed with a fluorescence microscope. (a) Image with normal light. (b) Image with $488 \mathrm{~nm}$ laser. Images with 10X magnification.

8.3 $\mathrm{M} \Omega$. A suitable threshold can easily be defined for either of these two conditions to make an automated decision regarding successful buffer loading and microchannel blockage without any visual inspection.

\subsubsection{Moving cells through the microchamels}

Cells from the fingerprint samples were transported from the sample reservoir towards the waste reservoir through the microchannel using a low electric field created with the application of 100 to $200 \mathrm{~V}$ through the electrode-pair, generating a low electric field below $25 \mathrm{kV} / \mathrm{m}$. As cells are electronegative by nature, they experience a pulling force towards the positive electrode due to Electrophoresis phenomenon [135]. As cells moved inside the microchannel, this applied voltage was withdrawn. Thus cells settled inside the microchannel. To verify the presence of cells inside a microchannel, some cell samples were dyed with a fluorescence cell marker, Alexa Fluor 488 Annexin V. Photographs were taken with the fluorescence microscope after positioning these cells inside the microchannel. A set of photographs (both optical and fluorescence) are presented in Figure 5.7, confirming the presence of a cell inside the microchannel. 


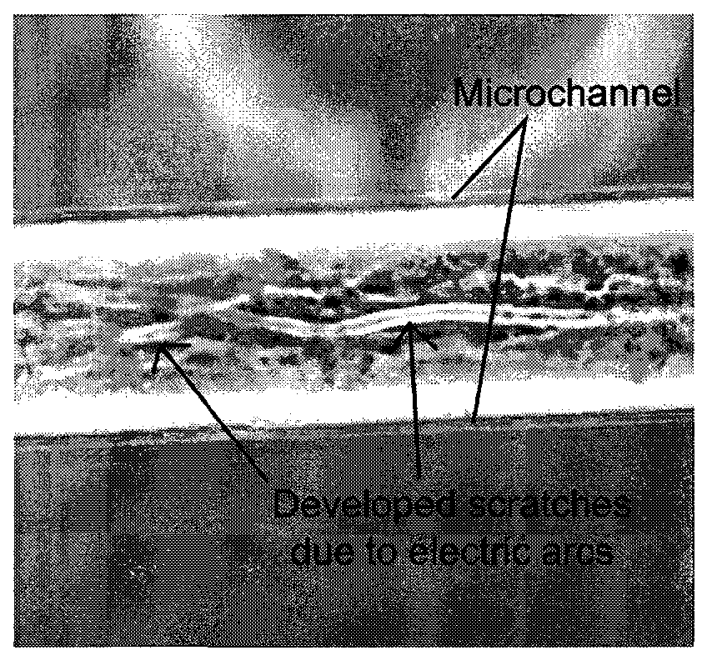

Figure 5.8 A microscopic image (10X magnification) of a section of a microchannel of the MFD showing scratches developed inside the microchannel resulted from electric arcs when $1.5 \mathrm{kV}$ was applied across the $8 \mathrm{~mm}$ long microchannel.

The movement of cells due to the Electrophoresis force, however, was not uniform. Cells tended to adhere to the channel walls while moving through the microchannels. Two factors might had contributed to this type of behavior. One of the factors was the surface irregularities of the microchannels. These irregularities of the channel surfaces were clearly observed in SEM photographs as shown in Figure 3.4 and Figure 3.5. The irregularities developed during to the chemical etch process of the fabrication technology. The surface irregularities were higher for the channel walls compared to the channel beds. The second contributing factor was the force developed due to Zeta Potential, defined as the electrostatic potential generated by the accumulation of surface charges along the silica based channel surfaces [27]. In addition, as a constant potential was applied during these experiments, Faraday's reaction at the electrodes caused formation of bubbles at the electrodes.

\subsubsection{Electrical lysis with a high electric field}

EL phenomenon was observed by applying a long pulse of 5 to 9 seconds durations of a high voltage through the electrode-pair. The range of the applied voltage for an 8 
Table 5.1 Observations under various electric field strengths

\section{Electric field range Observations}

About $25 \mathrm{kV} / \mathrm{m} \quad$ Cells moved due to Electrophoresis

Between 90 and $150 \mathrm{kV} / \mathrm{m} \quad$ Electrical lysis of cells were observed

Above $175 \mathrm{kV} / \mathrm{m} \quad$ Electric arcs were generated

Table 5.2 Average values of various parameters from the electrical lysis experiments ( $8 \mathrm{~mm}$ long microchannel)

\begin{tabular}{ll} 
Parameter & Value \\
\hline Applied potential & $800 \pm 100 \mathrm{~V}$ \\
Excitation time & $7 \pm 2$ seconds \\
Current flow & $0.5 \pm 0.2 \mathrm{~mA}$ \\
Power dissipation & $295 \pm 55 \mathrm{~mW}$
\end{tabular}


$\mathrm{mm}$ long microchannel was between $750 \mathrm{~V}$ to $900 \mathrm{~V}$. The critical electric field $\left(E_{c}\right)$ for $\mathrm{EL}$ was above $90 \mathrm{kV} / \mathrm{m}$. It was necessary to maintain the electric field just above the critical electric field, as electric arcs were observed with an applied potential higher than $1.5 \mathrm{kV}$ for a $8 \mathrm{~mm}$ long microchannel. The estimated electric field were higher than $175 \mathrm{kV} / \mathrm{m}$. These arcs, probably due to the dielectric breakdown of water, created scratches on glass surface inside the microchannels as observed in Figure 5.8. A summary of observations under different electric field strength and averaged values of various parameters are tabulated in Table 5.1 and Table 5.2, respectively. The average applied voltage of $800 \mathrm{~V}$ produced an estimated electric field of $100 \mathrm{kV} / \mathrm{m}$ inside the $8 \mathrm{~mm}$ long microchannel. Average time required for EL was recorded to be $7 \pm 2$ seconds from the time of the high voltage application. This time included the slow turn-on time delay of the power supply unit itself. The current flow, power dissipation and energy consumption were $0.5 \pm 0.2 \mathrm{~mA}, 0.295 \pm 0.055 \mathrm{~W}$ and $2.2 \pm 0.23$ Joules, respectively, for this microchannel length.

Figure 5.9 shows a temporal sequence of photographs during an EL experiment with the MFD. Figure 5.10 shows corresponding waveform of both channels as recorded in the oscilloscope. Here, the maximum load voltage (across the microchannel) was $766 \mathrm{~V}$, and the microchannel length, width and height were $8 \mathrm{~mm}, 80 \mu \mathrm{m}$ and $20 \mu \mathrm{m}$, respectively. The maximum current flow is calculated to be $0.65 \mathrm{~mA}(=6.5 \mathrm{~V} / 10$ $\mathrm{k} \Omega$ ). The microchannel (d) was defined by its intersection with a reservoir (c), not by an etch-pad (e), as discussed previously. Therefore, the microchannel (d) terminates at the intersection of the reservoir (c). The sample cell (a) inside the microchannel was exposed to a high electric field (about $100 \mathrm{kV} / \mathrm{m}$ ) and was lysed in about 6.5 seconds. The control cell (b) inside the reservoir was exposed to a lower electric field (as demonstrated through simulations in Figure 4.10) and was not lysed. This observation confirms that the developed electric field inside the microchannel is higher than that in the reservoir.

A temporal sequence of enlarged photographs of the sample cell (a) is shown in Figure 5.11. Careful observation of these photographs indicates that there was a 

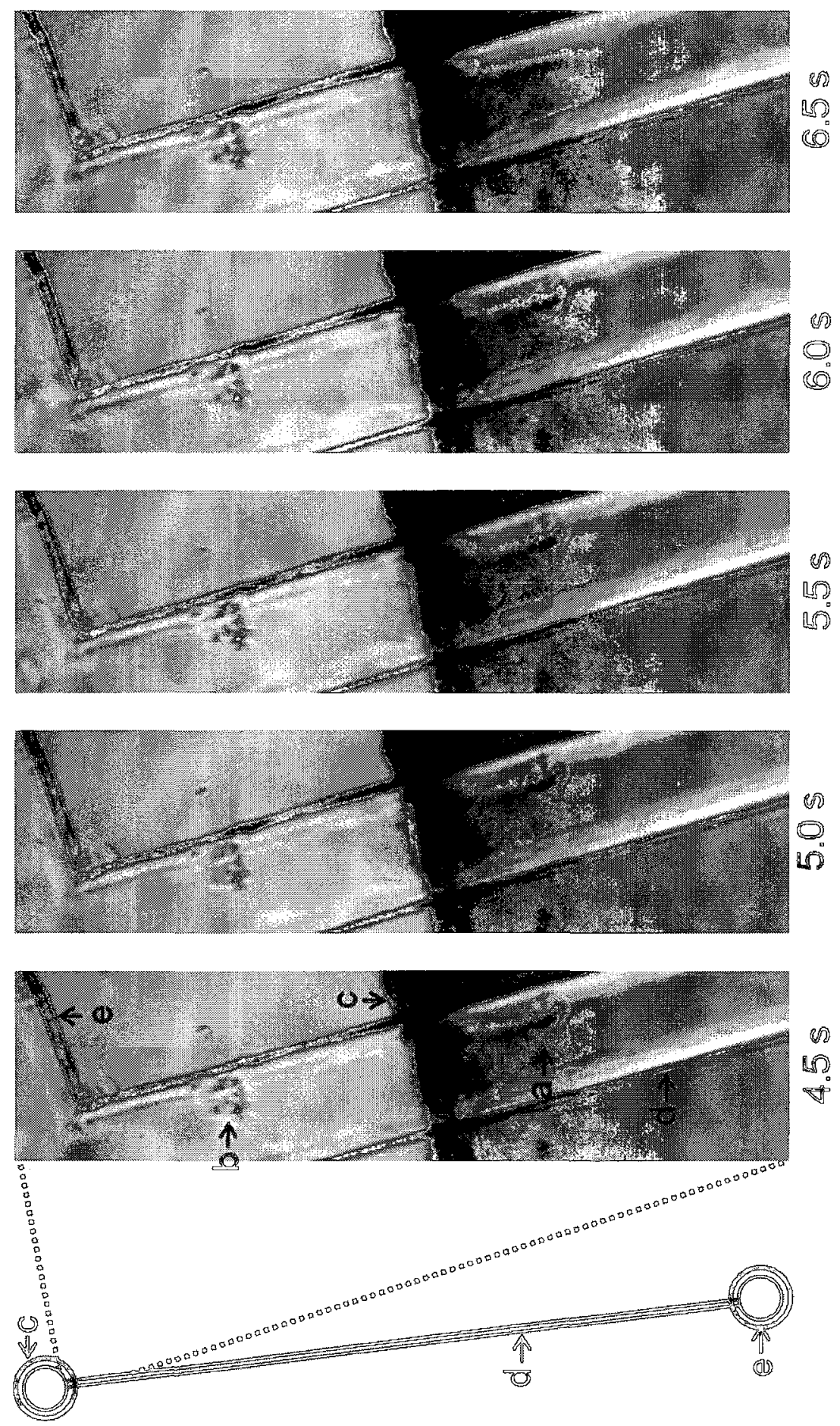

TIigure 5.9 Temporal sequence of microscopic images (10X lens) showing $E \mathbb{L}$ of a sample of fingerprint debris captured at a rate of 2 fps. Legends: asample cell exposed to a high electric field inside the microchannel, b-control cell exposed to $a$ low electric field in the reservoir, $c$ - the reservoir (i.e. access hole), d- the microchannel, e- the etch-pad. 


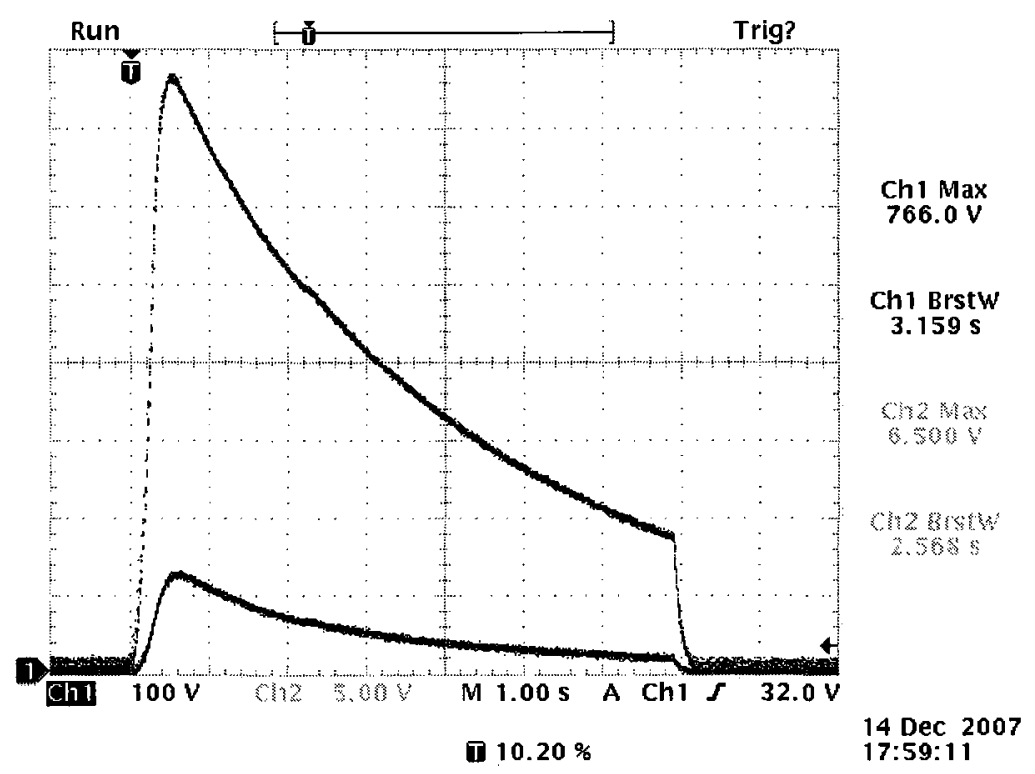

Figure 5.10 A snapshot captured from the oscilloscope during the application of a high voltage for EL experiments. Ch1: The applied voltage across the electrode pair $\left(V_{a p p}\right)$. Ch2: Voltage $\left(V_{R s}\right)$ developed across the series resistance, $R_{s}=10 \mathrm{k} \Omega$. $V_{R s}$ is proportional to current flow $\left(I_{c h}=V_{R s} / R_{s}\right)$ through the microchannel. Reader may refer to Figure 5.2 for the setup.

gradual decrease in contrast (intensity) of the sample cell (a) between 5 to 6 seconds, just prior to lysis. A logical deduction and explanation is that Electroporation (EP) phenomenon, that occurs prior to EL, had caused a reduction of the refractive index difference inside and outside of the cell. From these photographs, the duration of EP was less than one second. This time duration between initiation of EP and completion of EL was less than a second, which is comparable to other reports on EL $[64-66,82,83,94]$.

No chemicals were involved in the method except for D-PBS fluid, which controls the $\mathrm{pH}$ of the medium. D-PBS buffer fluid is not required to be removed from the lysate for further processing of Deoxyribonucleic Acid (DNA) detection. Withdrawal of the applied potential immediately after lysis is required to minimize degradation of lysate that might occur due to prolonged electric field stress.

This proof-of-concept device demonstrated that cells collected from human fingerprint samples can be lysed within a microchannel by an applied voltage of $750 \mathrm{~V}$ to 

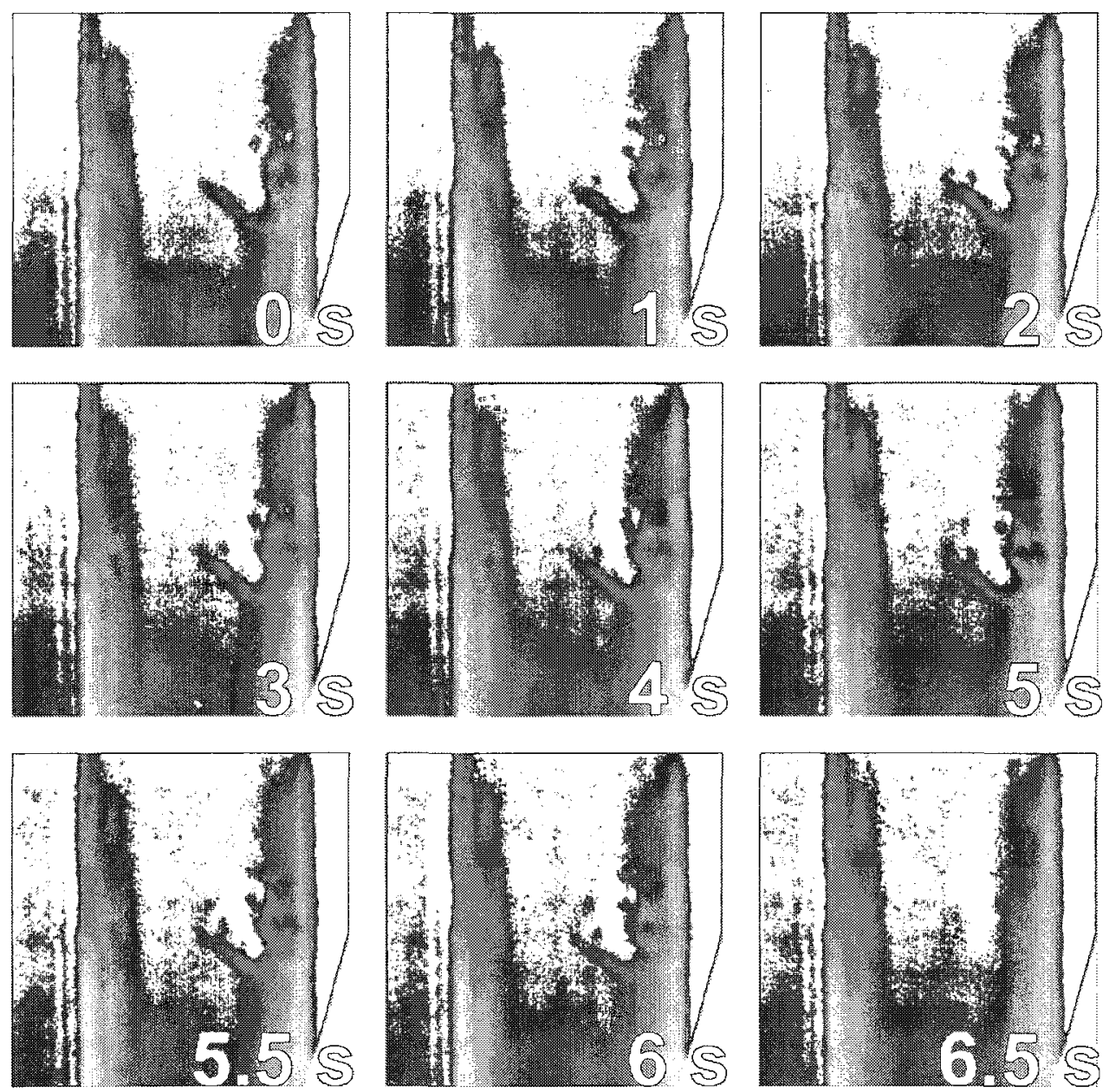

Figure 5.11 An enlarged version of the temporal sequence of photographs showing the cell (a) being lysed from the time of pulse application. 
$900 \mathrm{~V}$ in a very short amount of time (about 5 to 9 seconds). A low power consumption (about $300 \mathrm{~mW}$ ) was achieved using the microchannel structure. The device is small and reusable. The method is fast, simple and suitable for on-chip operation.

\subsubsection{Summary and discussion}

As a preliminary study, an MFD was designed and fabricated for rapid EL from a minute amount of non-invasively collected cell-samples from human fingerprints. Analytical expressions were developed and validated through Finite Element Method (FEM) analysis. It is shown that the analytical expressions produce reasonable accuracy as long as the length of the microchannels are larger than $2.5 \mathrm{~mm}$. For smaller length of microchannels, an extension of the analytical expression is proposed. Electrical characterization of microchannel was done through impedance measurement, equivalent circuit modelling and electric field analysis. It was observed that applying AC potential with $\mathrm{f}>10 \mathrm{kHz}$ reduces electrode degradation and bubble formation due to Faraday's reaction. However, such microchannels have significant (capacitive) reactive component, which becomes prominent for high frequency operation.

Moving cells through the microchannels using Electrophoresis technique showed practical limitations due to cell adherence tendency with the channel surfaces. This adherence tendency was a combined result of the surface irregularities of the microchannels and Zeta Potential developed due to surface charges on the silica based channel surfaces. EL phenomenon was observed by applying a high electric field of 90 to $150 \mathrm{kV} / \mathrm{m}$. Rapid lysis (about 7 seconds) and low energy requirement (about $2.2 \mathrm{~J})$ indicates potentials of EL. The temporal sequence of photographs from the experiments suggested that EP phenomenon occurring just prior to EL.

Comparing this device with other devices proposed for various other applications of EL [64-66, 82-85, 87, 91, 92, 94,97-100], following unique attributes are identified.

- The MFD was compatible with the standard microfluidic fabrication platform.

- The EL method is much faster compared to other methods of cell lysis. 
- The cell samples were collected non-invasively from human fingerprints.

- The device consumed very low amount of energy.

- The user can control the duration of the high electric field.

- The device was very simple, inexpensive and reusable.

- There is a potential for the method to be integrated within a chip with other microfluidic and electronic components.

There were several issues with the device and method, such as:

- Cells tend to adhere to the microchannel surfaces as they were moved along the microchannels. Other fabrication methods or materials might result in reduced adherence. Besides, larger channel cross-section will also reduce such adherence.

- The method required a high-voltage power supply. Reduction of the applied voltage is achievable using integrated electrodes.

- Bubble formation due to Faraday's reaction can be reduced by applying Alternating Current (AC) or pulses with microsecond duration. However, the critical time requirement for EL imposes constraints on the duration of pulses.

- Sometimes microchannels were blocked from debris as they were moved through the microchannels. This prevented buffer loading in the next experiment due to trapped air. To reduce such blockage in a reusable device, wider microchannel design is recommended, even though power dissipation will increase.

The analysis and experiments of this initial device enabled us to improve device design later in this research. To reduce or eliminate these issues, one device using Metal Multi-User MEMS Processes (MetalMUMPs) and two devices using Sensonit technology were fabricated. Findings with these devices is the topic of the next chapter. 
After the preliminary study, a second device was fabricated using MetalMUMPs that contained integrated electrodes. The purpose was to reduce voltage requirement for EL and reduce cell adherence. After that, devices using Sensonit technology were fabricated to reduce voltage requirement further. The MetalMUMPs-based device was designed at the end of 2006 and fabricated device was received mid of 2007. The Sensonit devices were designed at early 2008 and were received mid of 2008. In this chapter, we briefly discuss these devices, key findings, issues and expectations towards our research goal. Further experimental results and corresponding analysis is undergoing to complete this thesis.

\subsection{The Intermediate Device}

The intermediate device was developed using MetalMUMPs fabrication process. The fabricated device was tested in the fab lab of Department of Electronics, Carleton University, Ottawa.

\subsubsection{Excitation source setup}

The device to be tested required excitation ranging from $1 \mu \mathrm{s}$ to $10 \mathrm{~s}$ with magnitudes of $10 \mathrm{~V}$ to $40 \mathrm{~V}$. Pulse trains of 1 to 1000 pulses were to be generated with the setup. An Field-Programmable Gate Array (FPGA) was programmed for feasibility study. This section discusses briefly the setup of excitation source that consisted of two components: an FPGA board and a drive circuitry.

\subsubsection{Hardware programming}

An FPGA DE2 (Development Environment) board was programmed using Verilog programming language for excitation control and monitoring. This FPGA board is battery operable and is capable of performing various tasks involving control and monitor of a system. The program listing and relevant pictures are giving in Section 
C.1. As testing revealed issues related to biochemistry, further expansion of the FPGA-based testing platform was not pursued.

The board was programmed to operate in two modes: SET mode and RUN mode, selectable with a Double pole double throw (DPDT) switch. In SET mode, following four selections could be made:

1. Duration of ON time for each pulse.

2. Duration of OFF time for each pulse.

3. The number of pulses in a pulse train.

4. Amplitude of the pulse train.

The range of ON time and OFF time were from $1 \mu \mathrm{s}$ to $10 \mathrm{~s}$ for each. The range for number of pulses was from 1 to 1000 and the range for amplitude was from $10 \mathrm{~V}$ to $40 \mathrm{~V}$ (through the external drive circuitry, described latter in this section). All of these set parameters were stored in Random access memory (RAM) during program execution. The program instructions were stored in flash memory so that it can be loaded during boot-up. A set of default values were loaded for the set parameters during boot-up.

In the RUN mode, the program is on hold state unless a push button is pressed. One push button labelled START initiated the pulse train sequence using the set parameters stored in RAM (from the SET mode or default). After the pulse train finishes, the program returns back to the hold state. Another push button labelled RESET was provided to cease the pulse train immediately and reset all registers (not the set parameters), provided for the case of an erroneous situation. The excitation output was delivered to the drive circuitry through the General purpose input output (GPIO) port.

A 7-segment display consisting of 7 individual display units were used to let the user know the status of SET or RUN mode and various parameter values. Among the 7 display units, 3 were used to show the name characters of the SET parameter 


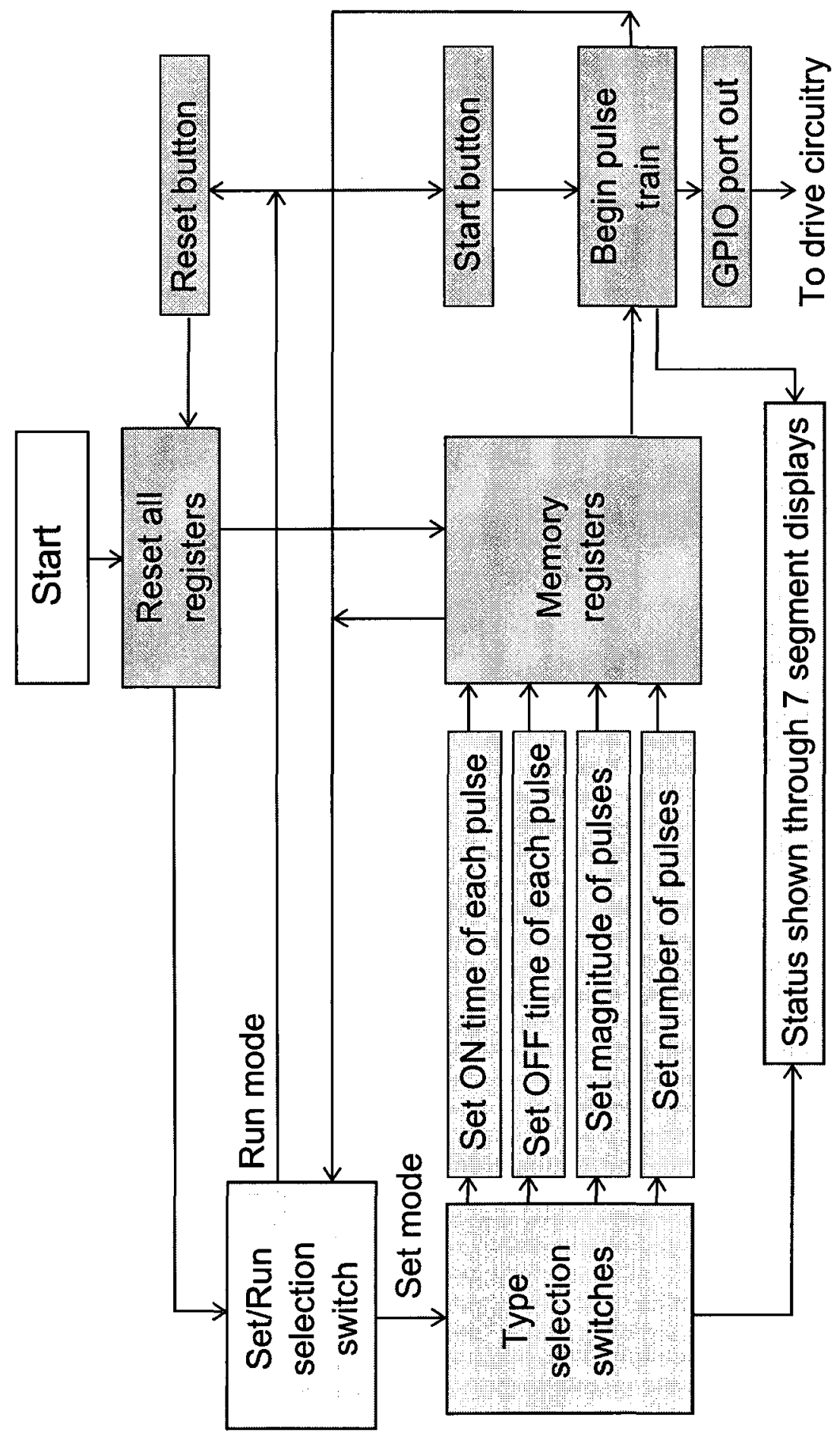

Figure 5.12 A block diagram showing major components and interactions of the blocks of the FPGA board to generate pulse waveforms of interest for testing of the device. 
(i.e. $[\mathrm{On}],[\mathrm{OFF}],[\mathrm{Am}],[\mathrm{rEP}])$. The parameter value was displayed using the remaining 4 display units. Here the text denoted as [.] signifies the display characters or digits.

The parameter value was displayed in the standard form (i.e. coef ficient $\times 10$ exponent). 2 display units were used to represent the coefficient (mantissa) digits in the form of $[\mathrm{x} \cdot \mathrm{y}]$ and the last 2 display units showed the exponent in the form of [ \pm e]. For example, a set parameter of $1 \mathrm{~ms}$ on time would be shown as [0n 1.0-3]. During the RUN mode, the display shows [Run] while in the hold state and [---] while delivering the pulse train.

The program was written using verilog programming language (hardware programming) in Register transfer level (RTL). Altera Quartus tool was used for this code development and design. The verification of the program was done with ModelSim (from Mentor Graphics). The program contained 3 modules: one module was for SET mode, one module was for RUN mode and the last module was for the top level.

\subsubsection{Drive circuitry for excitation}

The output of the FPGA through the GPIO port was fed into the drive circuitry. The drive circuitry consisted of a 8-bit Digital-to-analog converter (DAC), a current-tovoltage converter, a voltage converter and a push pull driver stage. The DAC converts the digital data from the GPIO port representing the amplitude of the excitation (using binary code) to the corresponding analog value. The output of the DAC chip was current, hence a current-to-voltage converter chip was required to follow the DAC. This converted voltage was fed to a variable voltage regulator chip as the control input. The output of the voltage regulator was $V_{s}$, that was provided as the supply voltage for the push-pull driver stage.

The schematics of the push-pull driver stage is shown in Figure 5.13. Two highvoltage transistors connected in complementary mode were driven by two complementary outputs of GPIO pins that delivered the pulse train. After the Device Under 


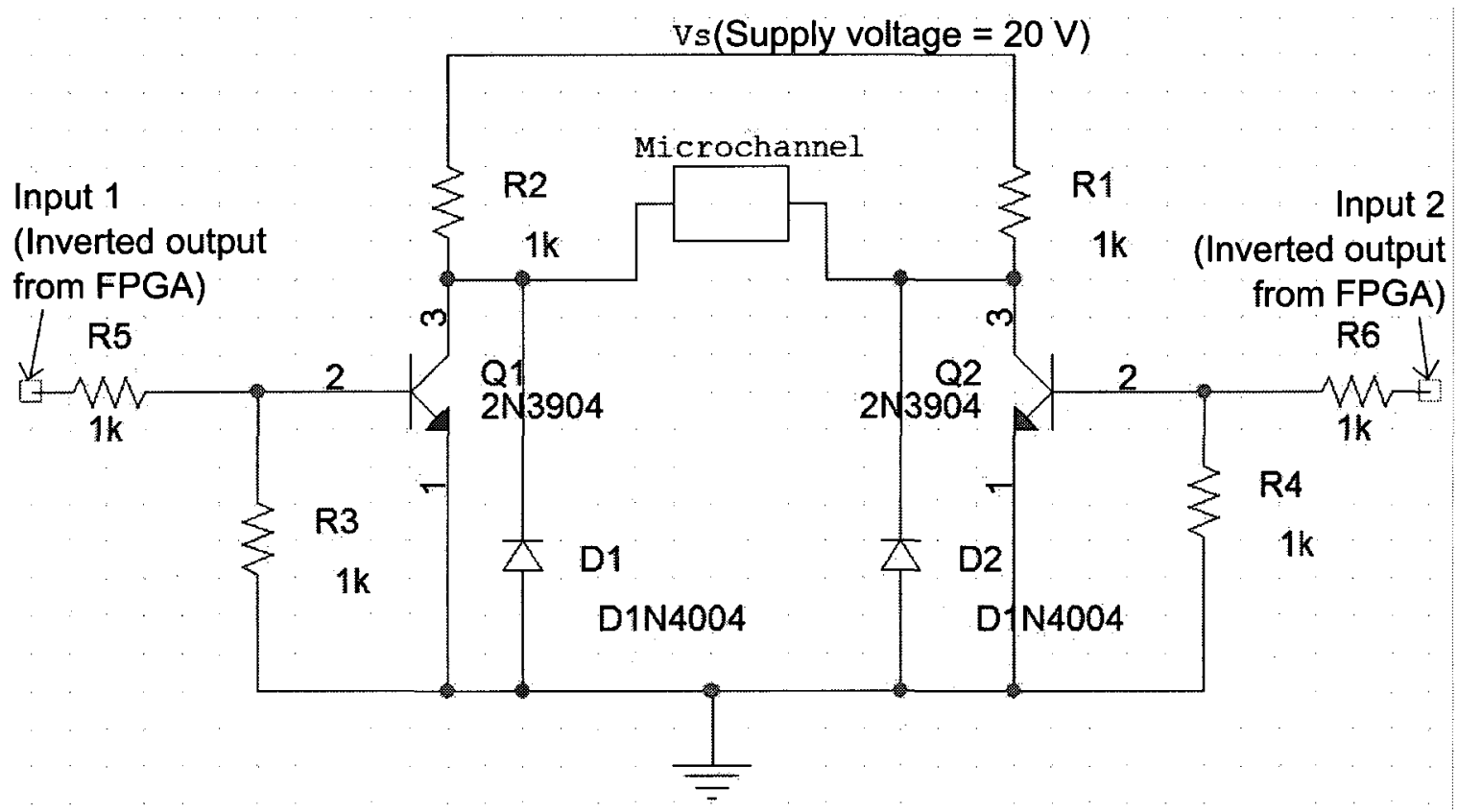

Figure 5.13 The schematics of the push-pull driver stage of the drive circuitry to operate the device (by exciting the microchannel) in bipolar mode.

Test (DUT) was secured on the stage of the microscope, the FPGA board and the drive circuitry were hooked up with the DUT before the experiment commenced.

\subsubsection{Fluidic encapsulation}

One major issue with the device was that the working area (reservoirs and electrodes) of the chip was not confined with any fluidic boundary. As the buffer fluid was introduced on the chip, fluids spread over the chip surface and came into contact with bond wires. The fluid reacted with the bond wires and decayed the wire even when no excitation were applied. This resulted in disconnected bond wires (Figure 5.14) and thus electrodes became unaccessible.

A solution to such unwanted flow of fluid is to encapsulate the device with a material that provides both fluidic and electrical insulation. An schematic representation of the intended encapsulation scheme for our device is shown in Figure 5.15. The area to be exposed for the chip (die) was $9 \mathrm{~mm} \times 9 \mathrm{~mm}$. Commercially available 

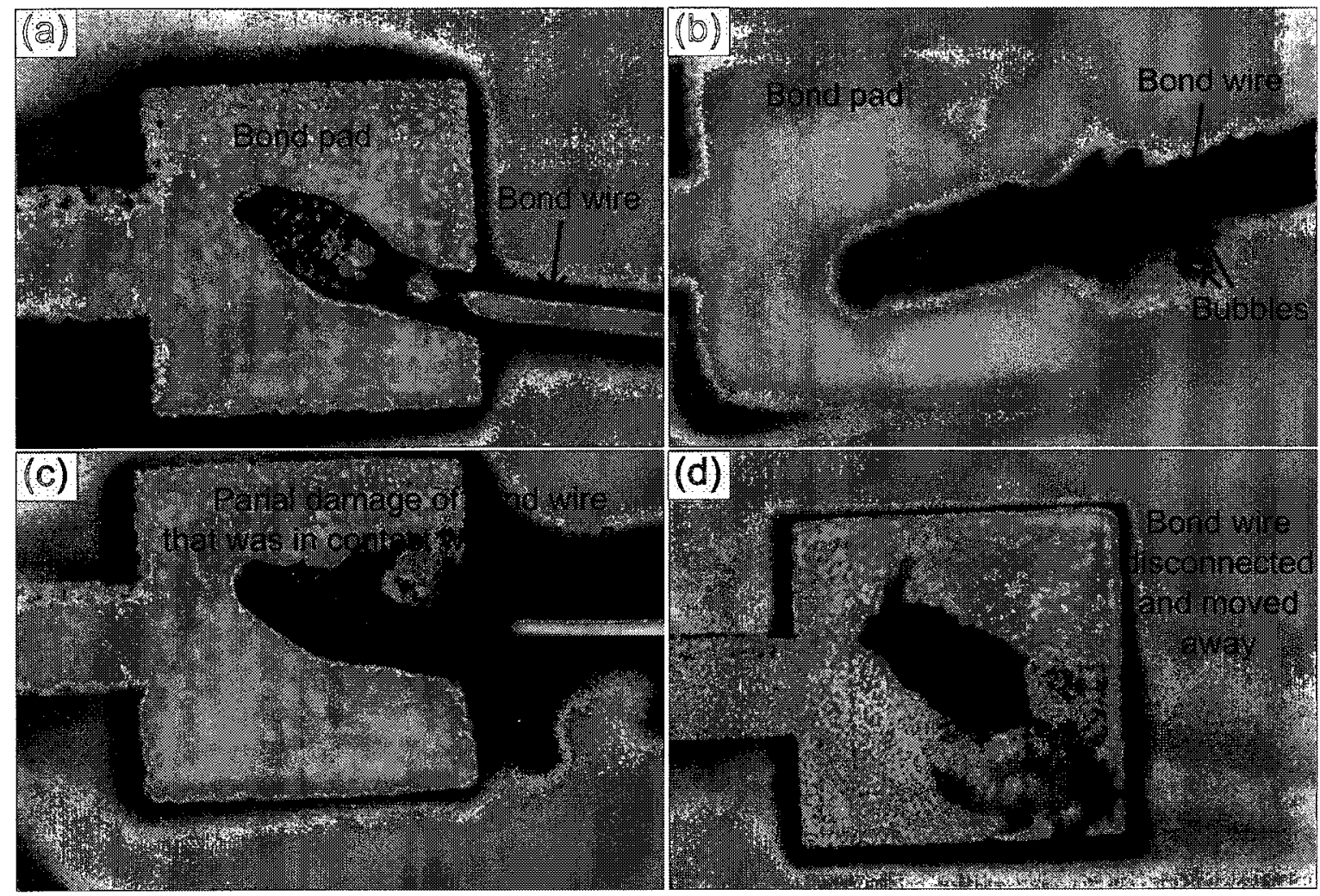

Figurre 5.14 Microscopic images with 20X magnification showing bond-wire degradation due to buffer fluid in the intermediate device. (a) A good bond wire connected to a bond pad. (b) Bubbles being formed as buffer fluid overflowed and came into contact with a bond wire. (c) A partially damaged bond wire due to reaction with the buffer fluid. Note that the portion of the bond wire in contact with the buffer fluid was damaged only. (d) A completely disconnected bond wire (moved away due to strain in bond wire). 


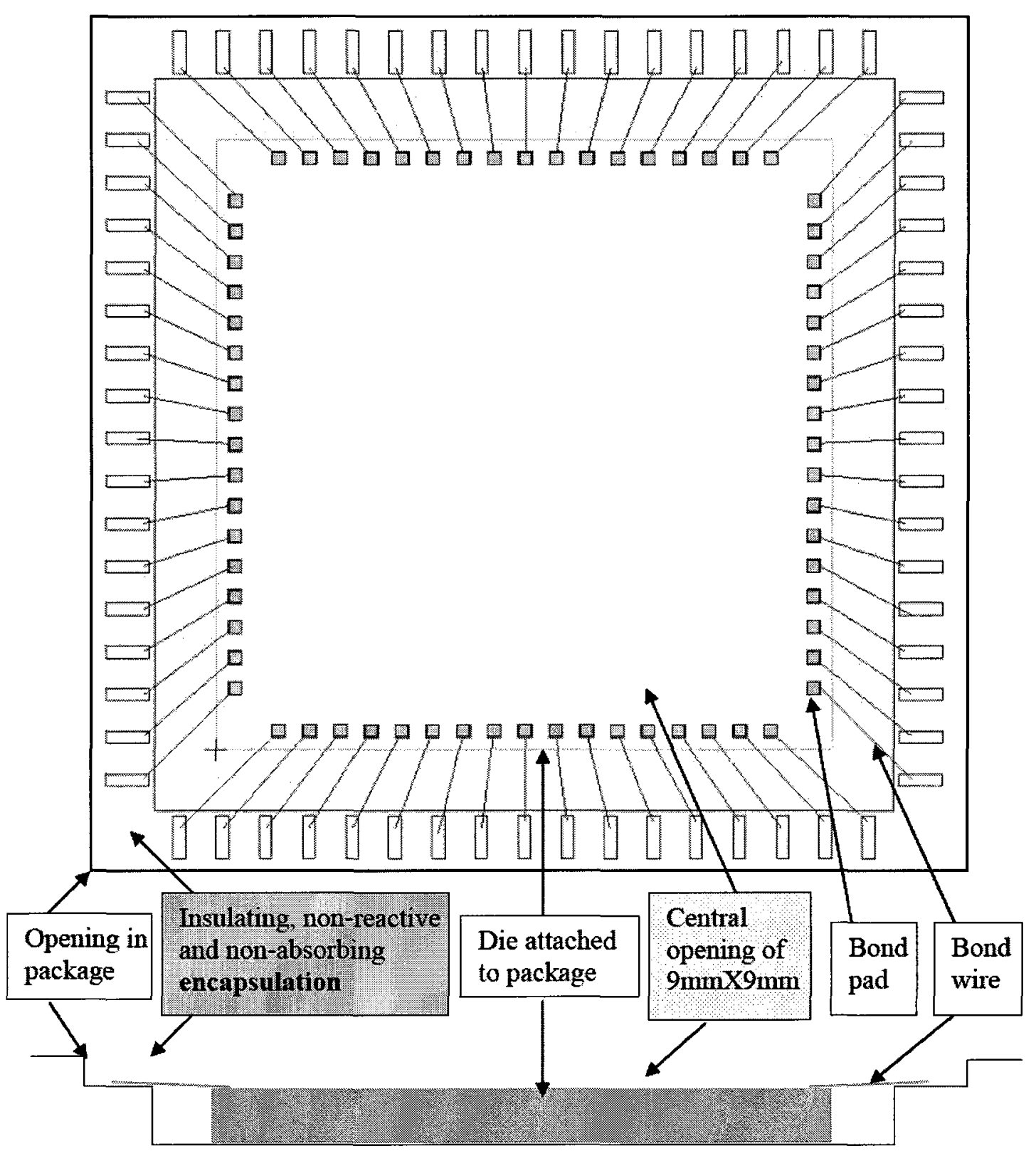

Figure 5.15 Encapsulation scheme for the device showing a top view (above) and a cross-section view (below). 


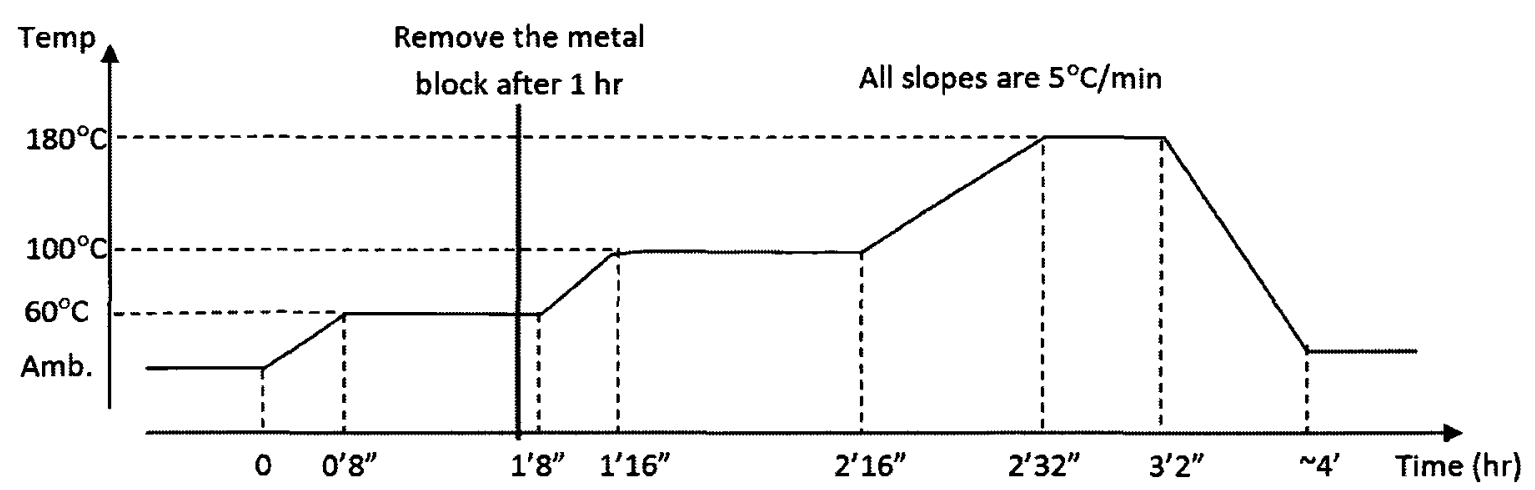

Figure 5.16 The heating cycle recommended for the encapsulation protocol using a metal-block in the application note from CMC. In the time scale, the symbol 'denotes hours and " denotes minutes of the elapsed time.

polyimide is one of the best suited solutions for such purpose, as it is available in fluidic state and can be solidified through a heating process called curing. We used high molecular weight polyimide of model PI-2611 from HD MicroSystems, NJ, USA. This polyimide is based on biphenyldianhydride/1,4 phenylenediamine (BPDA/PPD) backbone chemistry. It had following properties of interest:

- Viscosity: 110 to 135 Poise.

- Density: $1.082 \pm 0.012$ (fluid), 1.4 (cured) g/ $\mathrm{cm}^{3}$

- Moisture uptake: $0.5 \%$

- Glass transition temperature: $360^{\circ} \mathrm{C}$

- Coefficient of thermal expansion: $3 \mathrm{ppm} /{ }^{\circ} \mathrm{C}$

- Dielectric constant: 2.9 (at $1 \mathrm{kHz}, 50 \% \mathrm{RH}$ )

- Dielectric breakdown field: $>2 \times 10^{6} \mathrm{~V} / \mathrm{cm}$

- Volume resistivity: $>10^{16} \Omega \mathrm{cm}$

- Surface resistivity: $>10^{15} \Omega$ 


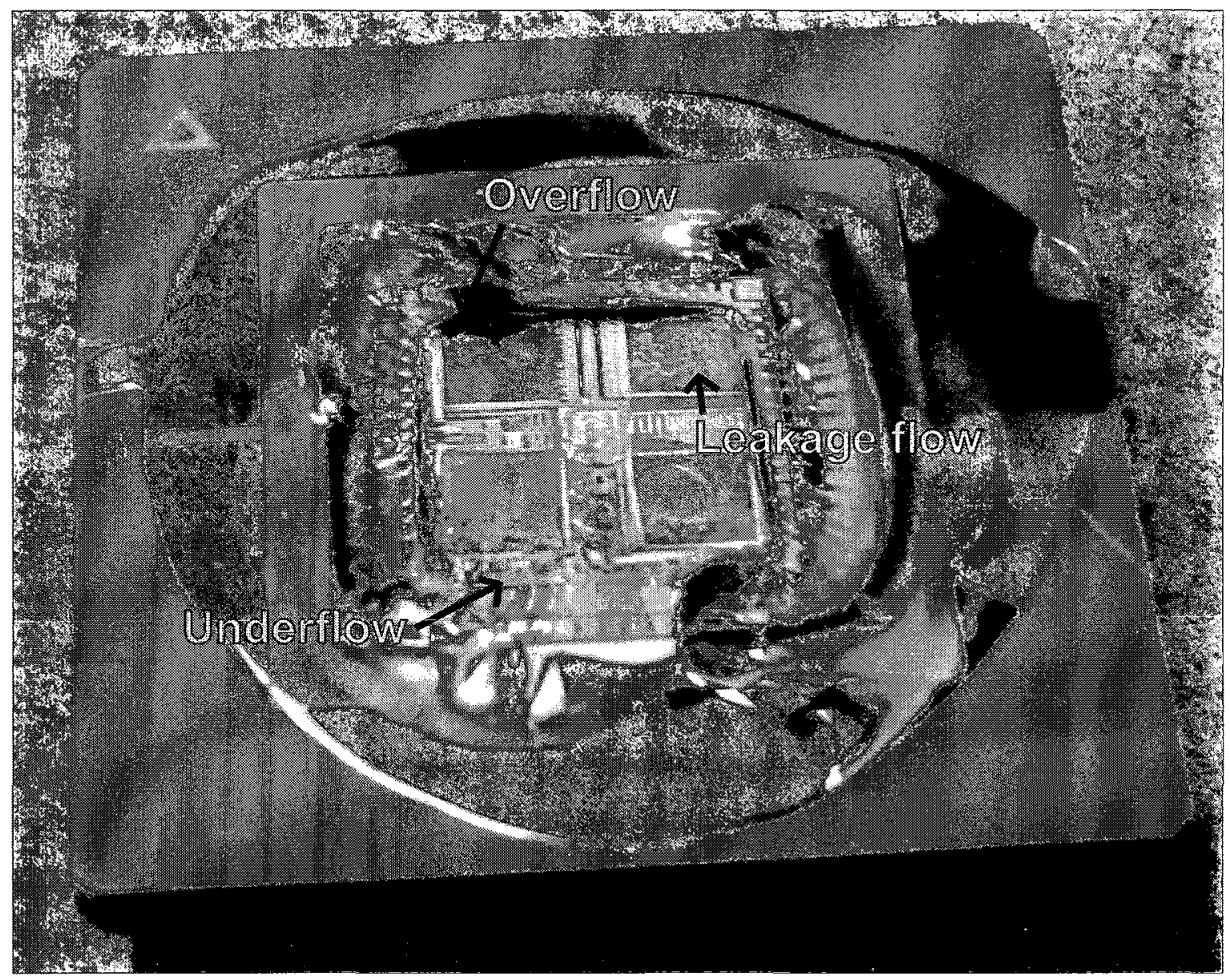

Figure 5.17 An encapsulation attempt with a metal-block according to the app-note from CMC showing different types of faults generated.

Polyimide is able to encapsulate layered structures like the device under consideration. In this device, the metal layer was about $20 \mu \mathrm{m}$ higher than the oxide layer on Si substrate. The electric and fluidic insulation properties of polyimide was satisfactory. Application notes (app-note) were obtained from CMC for such encapsulation. The best suited method available from these app-notes was to use a metal-block to cover the area to be exposed, and to apply polyimide around the metal-block. Then polyimide needs to be cured with a heating cycle (as shown in Figure 5.16) to solidify Polyimide. The metal-block had to be removed at the stage when the polyimide was transitional from fluidic state to solid state (indicated as the red line in Figure 5.16).

Several attempts to encapsulate using block-metals according to the procedures 
of the app-note resulted unsatisfactory results. Several types of faults regularly developed (Figure 5.17). These faults can be categorized in three groups as follows:

1. Overflow: This fault resulted from polyimide overflow into the chip as the metal-block was removed during the heating cycle as some polyimide could be still in fluidic state. This fault encapsulated part of the chip that was supposed to be exposed.

2. Underflow: This fault resulted from polyimide being lifted with the metalblock as it was removed during the heating cycle. This fault exposed some of the area that was supposed to be encapsulated.

3. Leakage flow: This fault resulted from the fact that the surface of the chip to be exposed was not plain (due to metal layers) and there existed certain gap between the bottom of the metal-block and the surface of the chip at various locations. Polyimide flowing underneath the metal-block before it became solidified resulted such faults.

We developed a new and simple protocol that achieved improved encapsulation with less complexity. A brief discussion of this developed protocol is discussed in Appendix Section B.1. Results obtained from the developed protocol was superior to previous attempts with the metal-block approach. An encapsulated device with developed protocol is shown in Figure 5.18. A section of the device observed under an optical microscope is shown in Figure 5.19, where sharp boundary between exposed and covered (encapsulated) regions is observed. The developed procedure minimized all the types of faults (overflow, underflow and leakage flow) results with the supplied protocol from CMC for fluidic encapsulation.

\subsubsection{Bubble formation and obstruction of visibility}

As an excitation was applied for EL, formation of numerous bubbles near or over the working region obstructed visibility (Figure 5.20). This bubble formation was more 


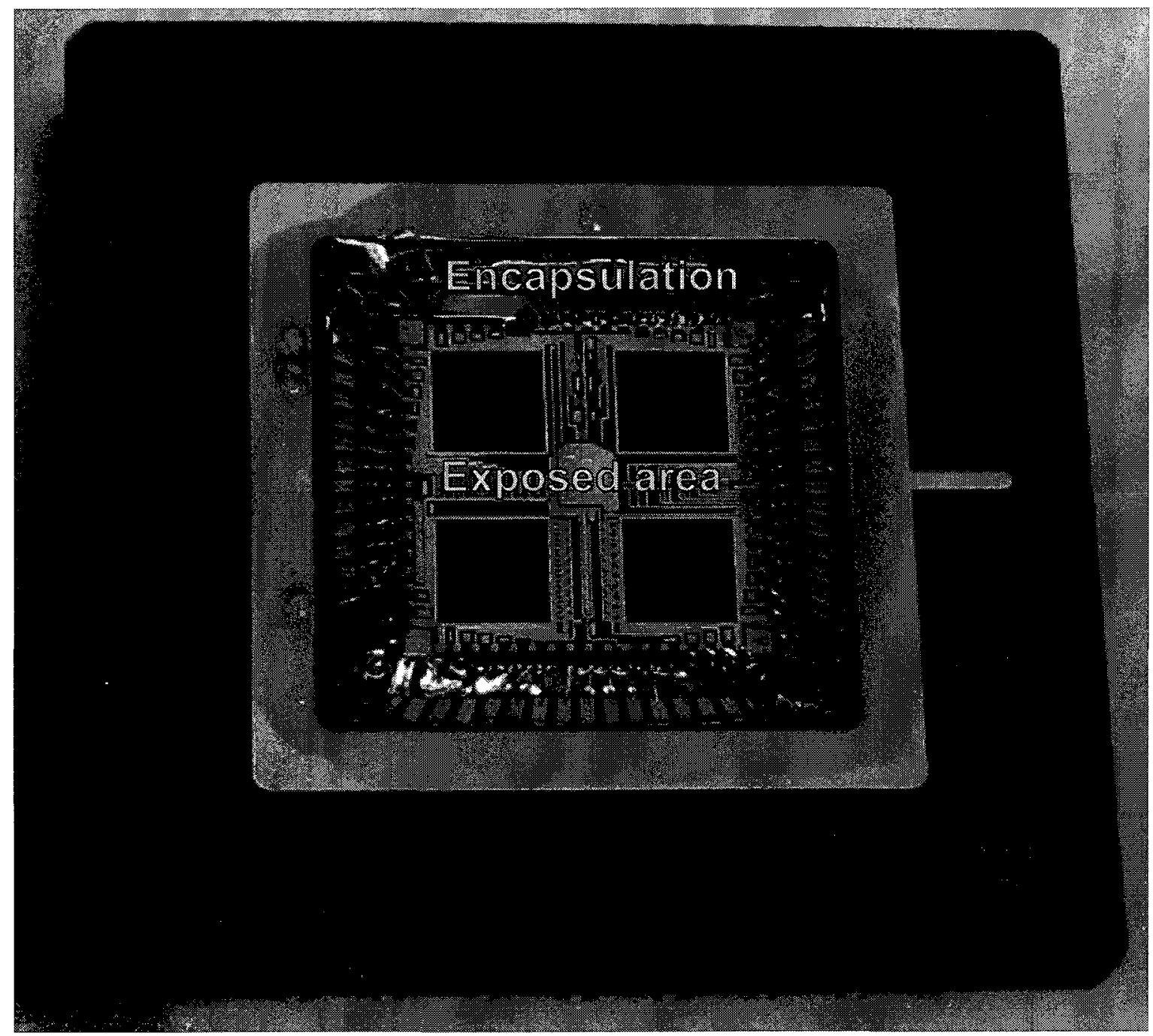

Figure 5.18 A photograph of the device after encapsulation with the developed protocol. The exposed area was free from polyimide leakage flow fault. The overflow and underflow faults were also minimal. 


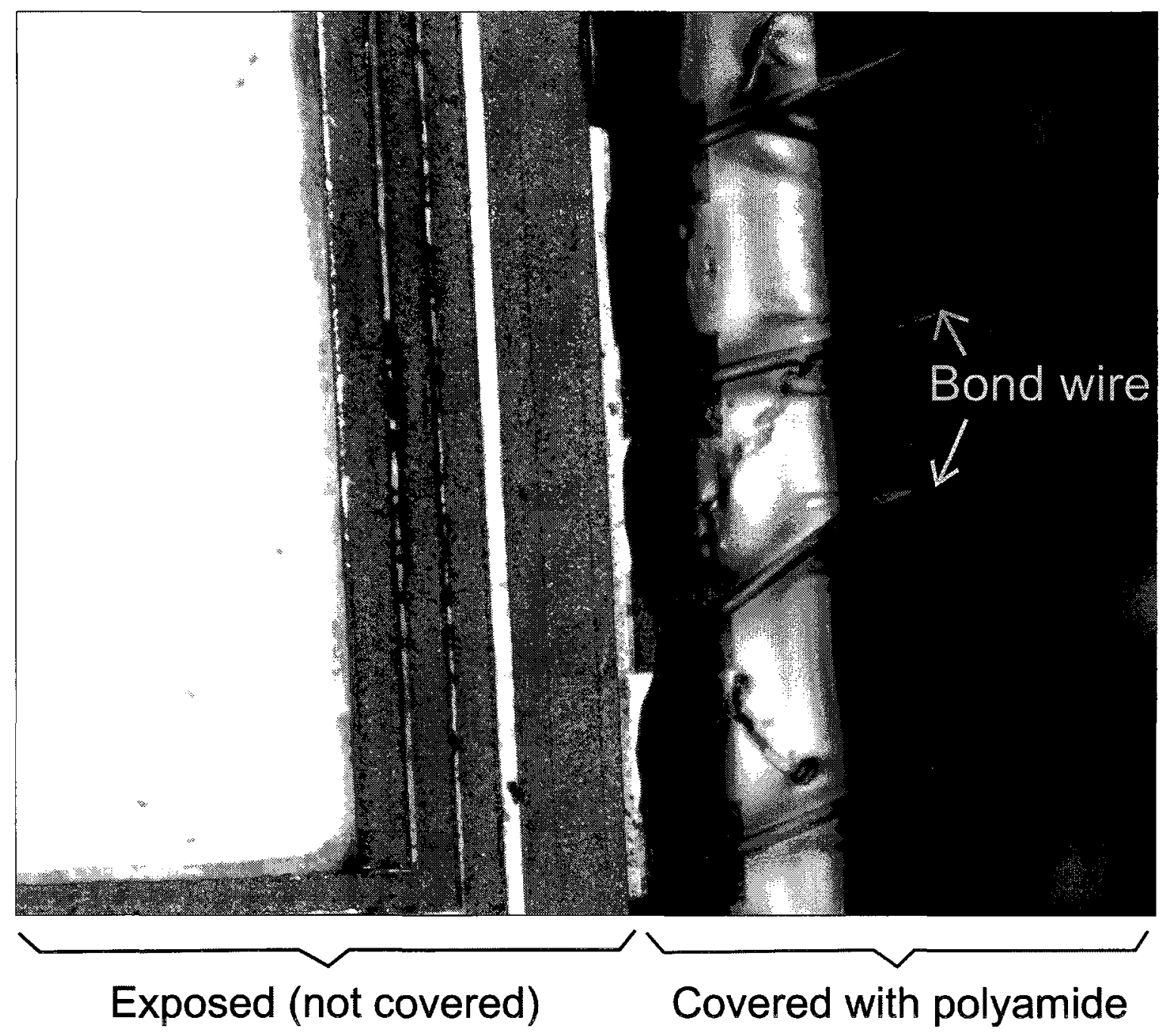

Figure 5.19 Section of the device after encapsulation with the developed encapsulation protocol observed under an optical microscope (10X magnification). The encapsulation boundary was sharply defined with minimal overflow or underflow fault. No leakage flow was noted. 


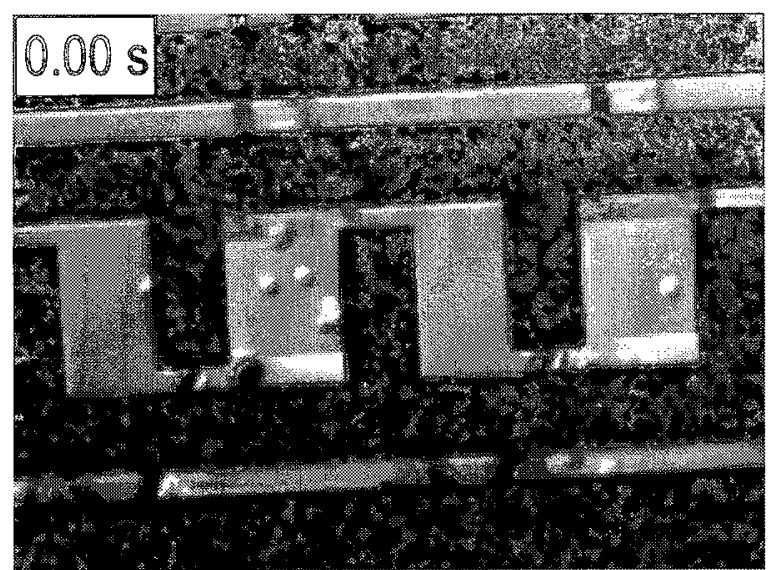

\section{$0.25 \mathrm{~s}$}
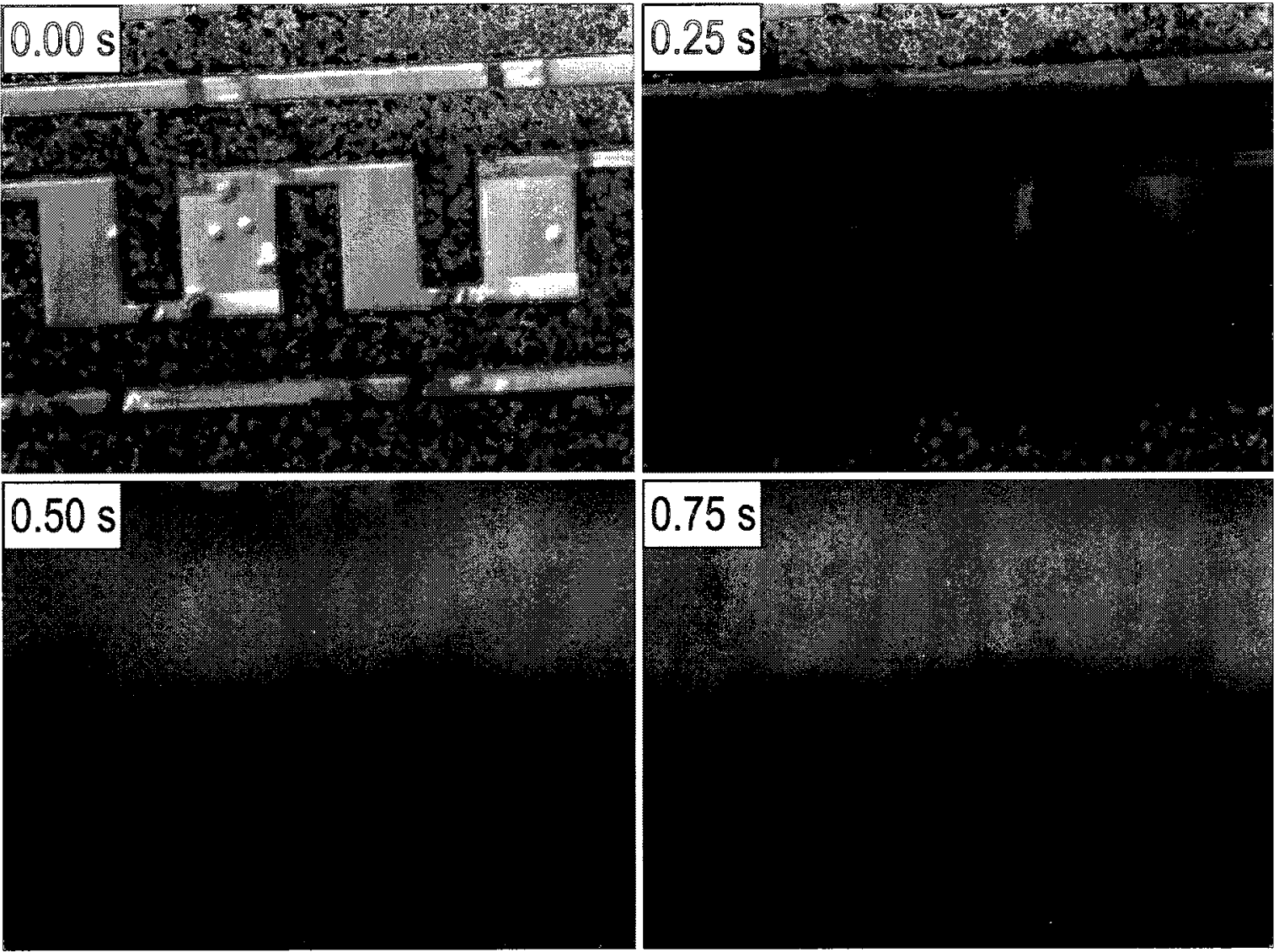

Figure 5.20 A pulse of 5 second duration of $20 \mathrm{~V}$ magnitude was applied to Electrode Set 4 b. Observability was obstructed due to formation of numerous bubbles. Microscopic images captured at $20 X$ magnification. 
pronounced for longer pulses ( $1 \mathrm{~s}$ or longer), that was required for EL. For smaller length pulses (1 ms or less), bubble formations were less; however, these durations were not sufficient for EL.

\subsubsection{Experimental results of electric pulse excitation}

Pictures obtained before excitation and after bubble disappearance showed reduction of number of debris or morphological changes (deformation) for longer (5 to $10 \mathrm{~s}$ duration) pulses with sufficient electric field strengths, suggesting possibility of EL and EP (Figure 5.21). Decrease in number of debris was associated with disintegration of membranes through EL. Decrease of contrast and morphological changes were associated with stress on membranes, pore formations and fluidic pressure due to permeable membranes resulted from EP. The voltage range required for a $20 \mu \mathrm{m}$ electrode gap was between 15 to $20 \mathrm{~V}$. With smaller pulse widths only morphological change was observed, but number of cells were not reduced, suggesting possibility of EP only. For example, as observed in Figure 5.22, one of the debris changed morphologically with $20 \mathrm{~V}$ for $1 \mathrm{~ms}$ duration across electrode gap of $100 \mu \mathrm{m}$. However, these results were not conclusive.

\subsubsection{Unresolved issues}

Other issues related to testing of this device was cell positioning issue and buffer evaporation issue. As the fluid flowed freely on the chip, it was difficult to position cells within the area of high electric fields. Besides, as the working area was exposed, buffers evaporated within several minutes, limiting available time to complete all relevant experimental procedures. 

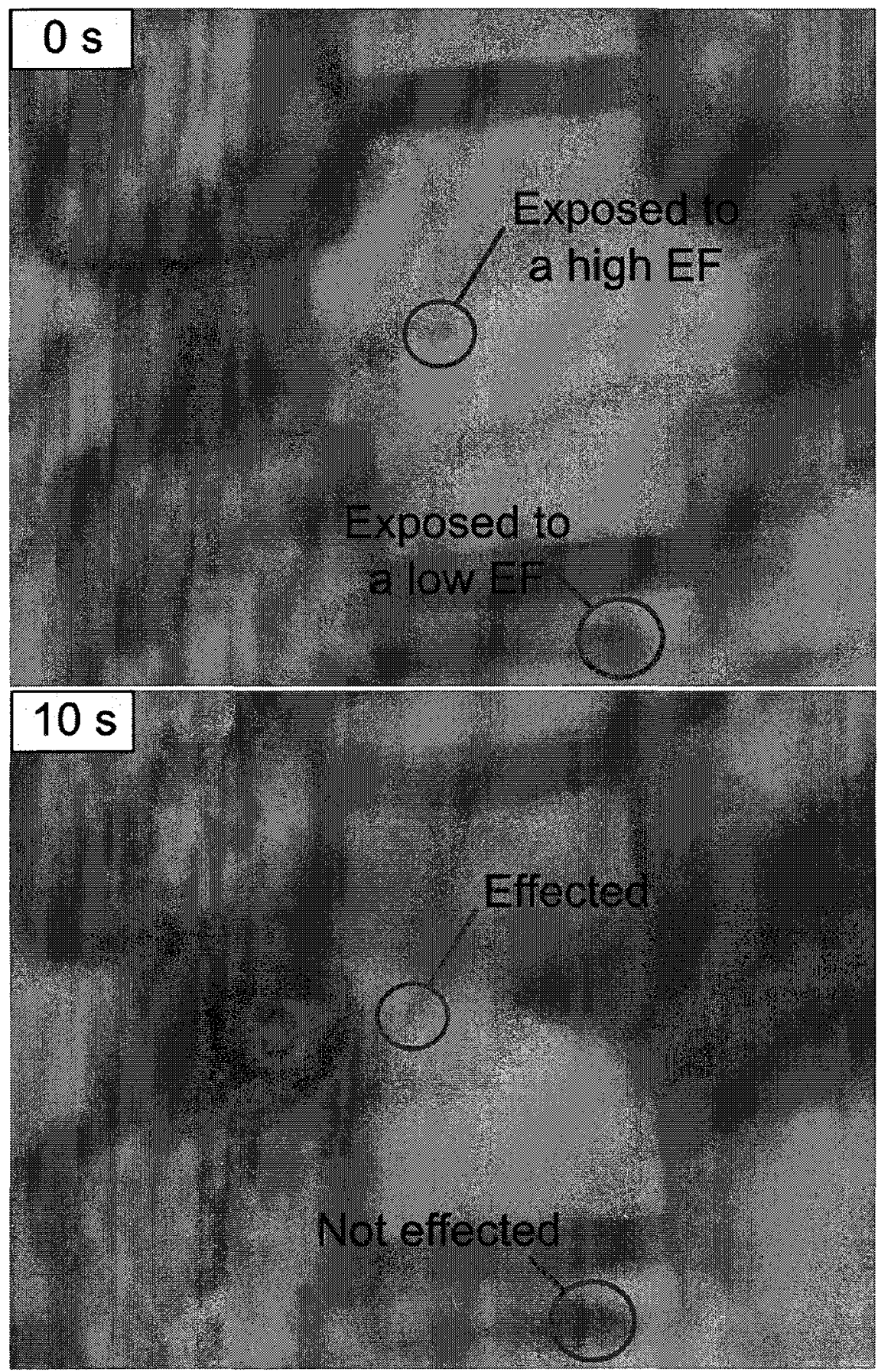

Figure 5.21 A set of microscopic images (40X magnification) showing morphological changes on fingerprint debris from an electrical pulse excitation. Here, an excitation voltage of $20 \mathrm{~V}$ was applied for 5 seconds. Note that only the debris at the high electric field region was affected. 

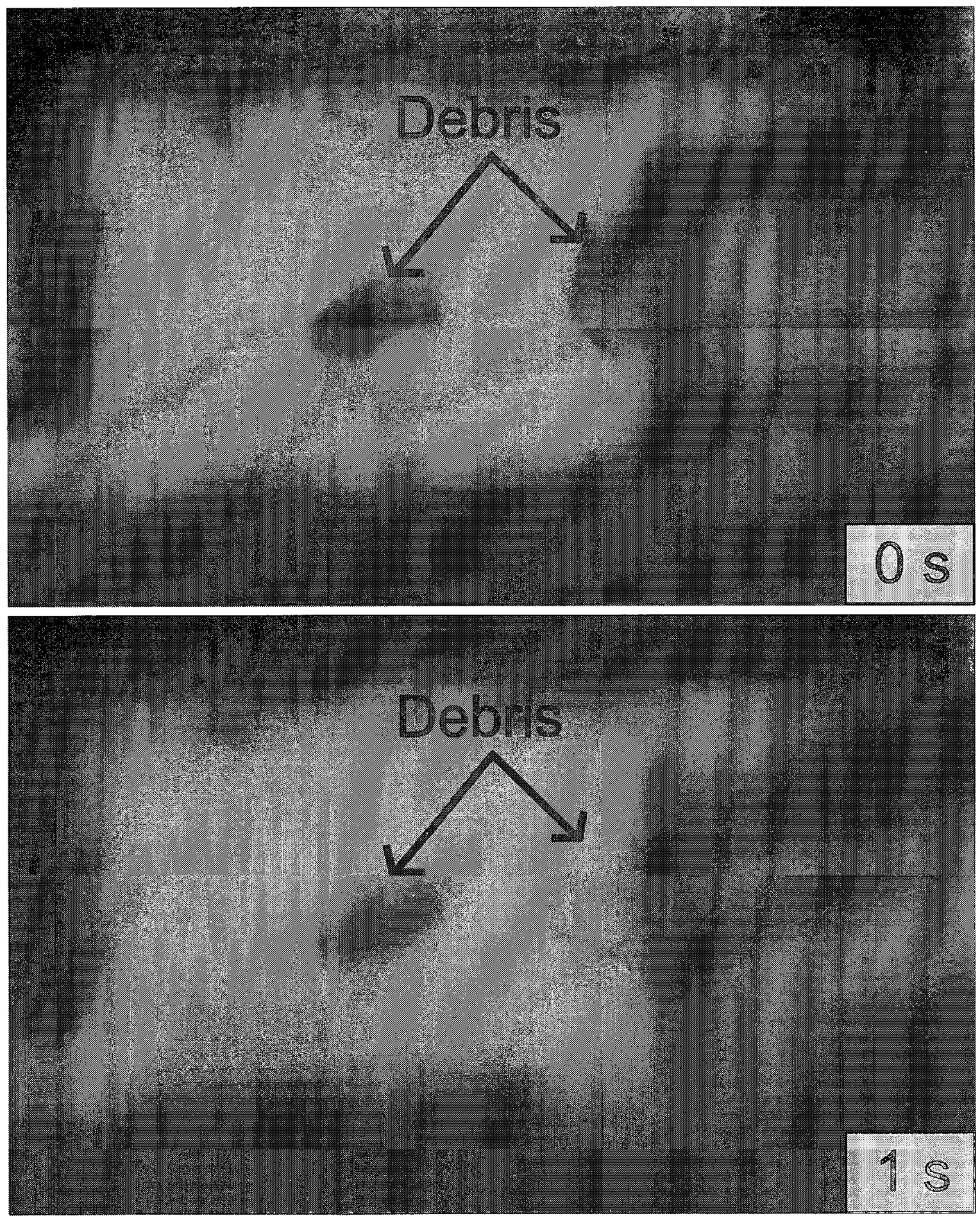

Figure 5.22 Microscopic images (40X magnification) showing effect of a pulse of $1 \mathrm{~ms}$ duration and $20 \mathrm{~V}$ magnitude application to the Electrode Set $4 \mathrm{a}$ with $100 \mu \mathrm{m}$ electrode gap. One of the debris was significantly deformed morphologically. 


\subsubsection{Discussion}

We have designed a MetalMUMPs device to resolve some of the issues with our initial device, such as high voltage requirement, cell adherence to channel walls and channel blockage from debris. However, we have encounter several new issues like fluidic encapsulation, bubbles obstructing visibility, cell positioning, buffer evaporation etc.

Encapsulation issue was imported to address immediately as the buffer fluid flow towards exposed bond wires and came into contact. The buffer fluid was reactive to the bond-wire material and damaged bond-wire through erosion, resulting disconnected bond wires. This required custom post-processing for encapsulation of fluid, so that the fluid is confined within a certain area on the chip. The area to be exposed for our chip was $9 \mathrm{~mm} \times 9 \mathrm{~mm}$. Using procedure given in the application note for such encapsulation resulted overflow, underflow and leakage flow faults. We have developed a protocol for encapsulation that yielded superior results and reduced all three types of faults. Such polyimide encapsulation allowed restricted the fluid flow in unwanted areas.

Bubble formation during long (1 second or more) excitations obstructed visibility. However, observations after bubble disappearance showed reduction in numbers and morphological changes of debris, suggesting EL and EP. Smaller pulses (1 ms or less) resulted only morphological change of debris, suggesting EP. However, the device did not yield conclusive results. Our experience from this device helped us to design the next device using Sensonit technology, which is described in the next section.

\subsection{The Final Device}

The final device was developed using Sensonit fabrication process. The fabricated device was tested in the labs of Cardiovascular Device Division of Ottawa Heart Institute, Ottawa. 


\subsubsection{Measurement of impedances}

We can estimate the electrode resistance using the sheet resistance data and some approximations. The sheet resistance data was provided by Micronit Corp., the fabrication foundry of the device. The sheet resistance of the integrated electrodes are $1.1 \Omega$ per square when inside microchannels (such as fingers), and $1.2 \Omega$ per square when enclosed by top glass slide (such as thick electrodes). To calculate the resistance of a rectangular section of electrode, we can use the following expression [131]:

$$
R=R_{\square} \frac{l}{w}
$$

where $R$ is the resistance of the path, $R_{\square}$ is the sheet resistance, $l$ is the length of the section, and $w$ is the width of the section. We assume that the resistance from the electrode port to the mid point of the central interdigitated electrode (finger) gives the average electrode resistance. We also assume that the total resistance of two section of electrodes can be approximated by a series combination of individual section resistances, irrespective to their configuration or orientation.

The length and width of the thick electrode under the electrode holes are 0.8 $\mathrm{mm}$ and $1.2 \mathrm{~mm}$, resulting a resistance of $0.8 \Omega$. The length and width of the thick electrodes on both sides of the microchannels are $1 \mathrm{~mm}$ and $8 \mathrm{~mm}$, producing $0.15 \Omega$ resistance. The length of the thick electrode from one side of a microchannel to the middle of the cell lysis chamber is $6 \mathrm{~mm}$ and corresponding width is $0.4 \mathrm{~mm}$, gives $18 \Omega$ resistance. Now considering mid point of a finger of interdigitated electrodes, that is exposed to microchannels. The length is $0.25 \mathrm{~mm}$ and width is $0.01 \mathrm{~mm}$, resulting a resistance of $27.5 \Omega$. Hence the total average resistance (assuming a series combination of all these resistances) is $46.45 \Omega$.

To verify this simple calculation, we have measured the actual resistance in different cell ports with respect to both electrode ports using an ohm-meter and tabulated average data as shown in Figure 5.23. The average resistance for the electrodes were $46.35 \Omega$ and $47.25 \Omega$, within $2 \%$ range of the simple calculation result. 


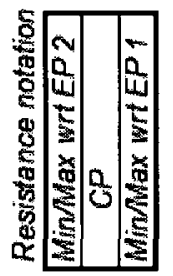

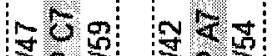

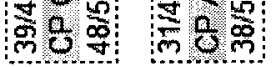

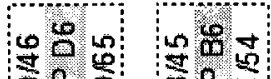

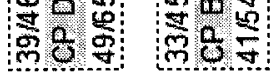

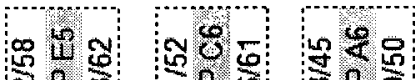
응

6. क क

궁ㅎㅇ

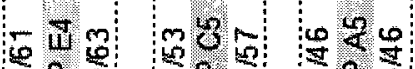

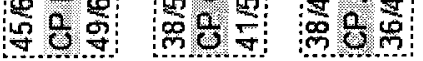

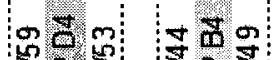

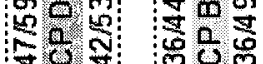

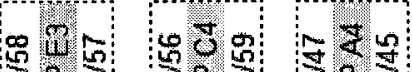
형

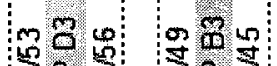

fo f :

때 영 $6 \circ 5$

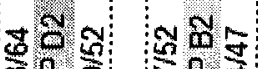

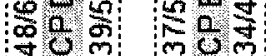

- 뜬 워워

영의

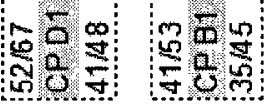

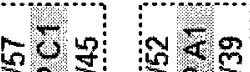

क웡

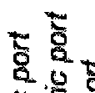

की

㩆离完
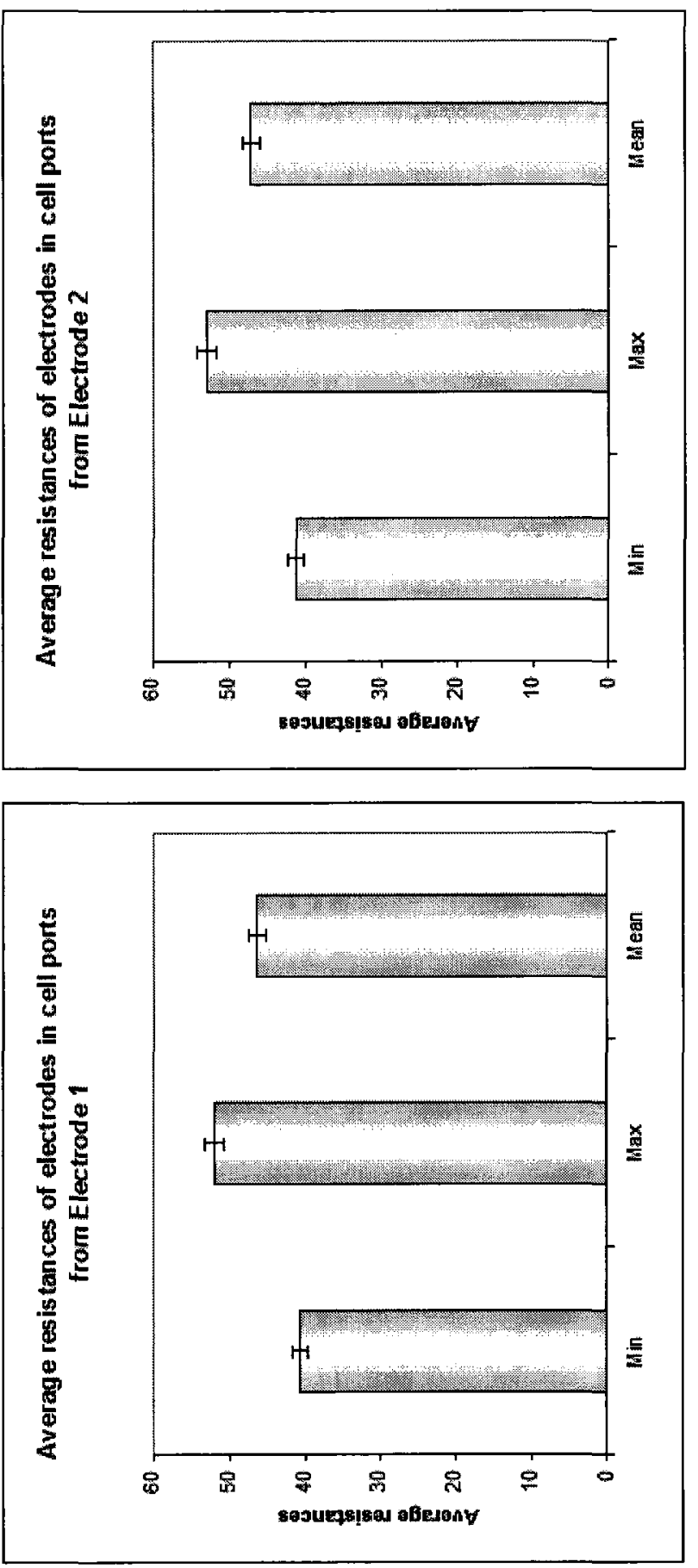

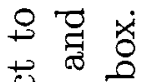

ஓ

변 웡

$3 \stackrel{0}{0}$

赵 $\frac{\Phi}{0} \cdot \frac{D}{\omega}$

ค م

च छ्र

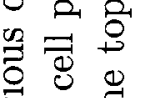

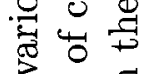

릉 뭉

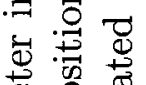

미을

ह.

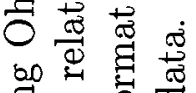

总 品

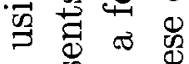

号怘寻

需焉

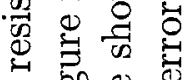

ข

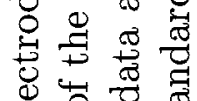
记

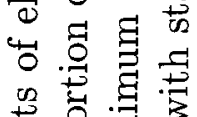

骂苛.品

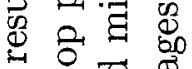

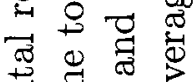

至皇

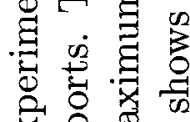

I

ஸे

10 总

일 0

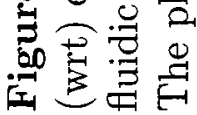




\subsubsection{Post-processing of the device}

The device required several post-processing to be done. Nanoport assembly (Upchurch Scientific, USA) were fixed on top of the fluidic ports for fluidic access with capillary tubing connections. Nanoport assembly composes of a port-base, a O-ring, and an adhesive ring. The derived protocol for fixing a nanoport assembly on a fluidic port of the device is given in Appendix Section B.3.

Electrode ports were cone-shaped holes of $1.5 \mathrm{~mm}$ diameter at the top and 0.7 $\mathrm{mm}$ diameter at the bottom. External electrodes were needed to be connected to the integrated electrodes at the bottom of the electrode ports through these holes. After testing several methods, Silver Conductive Epoxy (Cat. 8331-14G, MG Chemicals, ON, Canada) method was found to provide the best performance in terms of bonding strength and reliability. The protocol is outlined in Appendix Section B.4. Another protocol was developed to repair the silver epoxy bonding if the contact with epoxy and the integrated electrode under the electrode-port has high resistivity B.5. The device would be fixed on a clean glass surface to enable proper positioning on the microscope stage B.6.

A photograph of the device after post-processing is shown in Figure 5.24.

\subsubsection{Staining of freely flowing cells}

For experiments of this device, we have used cheek cells collected from inside of mouth using sterile buccal swabs as the non-invasive cell sample. Cheek cells are also non-invasive cell samples like fingerprint cell debris, but allowed us to have more number of cells per sample that was required for staining used in this device testing. Results obtained with this cell samples, however, would also be applicable to other cell samples, and we expect similar results would be obtained with fingerprint debris.

The sample cells were stained using Hematoxylin and Eosin as described below.

Staining using Hematoxylin: Hematoxylin colors basophilic structures with blue-purple hue. The basophilic structures are usually the ones containing nucleic 


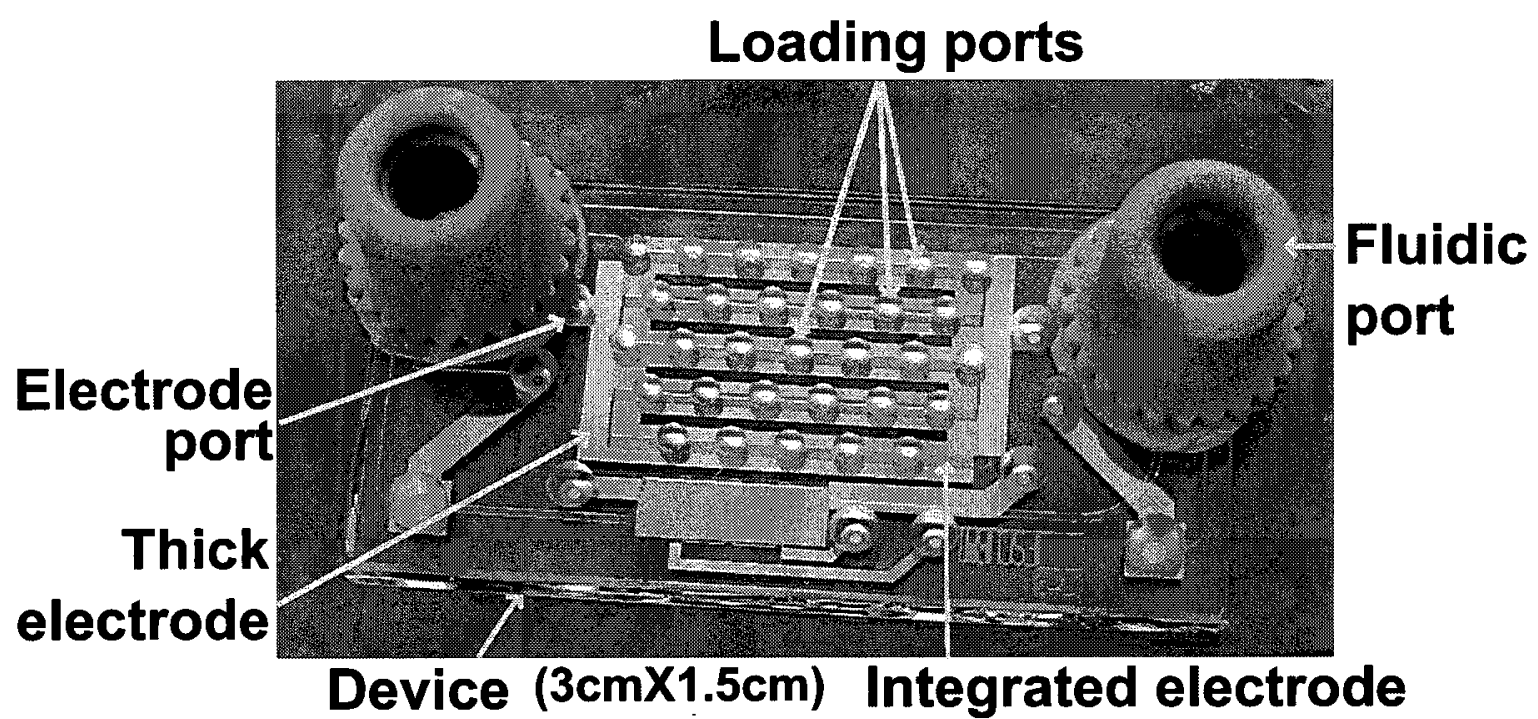

Figure 5.24 A photograph of the developed rapid electrical lysis microdevice using SensoNit microfluidics technology. The image taken after postprocessing shows some key components of the device.

acids, such as the chromatin-rich cell nucleus, the ribosomes, and the cytoplasmatic regions rich in RNA.

Staining using Eosin Y: Eosin Y colors eosinophilic structures bright pink. The eosinophilic structures are generally composed of intracellular or extracellular protein. The Lewy bodies and Mallory bodies are examples of eosinophilic structures. Most of the cytoplasm is eosinophilic and stains in pink color by Eosin. Red blood cells are stained intensely red.

Haematoxylin and Eosin (H\&E) staining: The combined staining of Hematoxylin and Eosin is called Hematoxylin and Eosin (H\&E) staining and is used frequently in histology to examine tissue. But all protocols for such staining assumes that the cells are fixed on a surface, which is not the case in our experiments as we need the cells to flow through the device.

After several trial-and-errors, we have developed a novel method for cell staining with $\mathrm{H} \& \mathrm{E}$ protocol for freely flowing cells that replaces conventional washing step with several centrifuge steps. The developed protocol is outlined in Appendix Section B.2. Photographs obtained with optical microscope of cells collected using 
buccal swabs (cheek cells) stained using the developed protocol is shown in Figure 5.25. The pink/red color is cytoplasm that binds to Eosin stain and the blue color interior is nucleus as chromatin (stands of DNA that is packed inside nucleus) binds to Hematoxylin stain. The stained cells did not lose stain even after 1 week. For experiments, we have used $\mathrm{H} \& \mathrm{E}$ stained cells that were stained within 1 week or less.

\subsubsection{Chemicals and equipment}

Experiments were performed in various buffers, such as D- $\mathrm{H}_{2} \mathrm{O}, \mathrm{D}-\mathrm{PBS}$, and isotonic, hypotonic and hypertonic solutions. For wetting of the DUT for the first time, $10 \%$ detergent solution was used only once and washed thoroughly. No other chemicals were used in the procedure.

As the source of excitation, a pulse generator (HP 33120A, Hewlett Packard, USA) was used. An oscilloscope (TDS-3012, Tektronix, USA) was used to record the pulse waveform. A capacitor (C) was included in series of the pulse power supply, which enabled two-fold advantages:

1. It prevented accidental damage of the device by connecting a Direct Current (DC) voltage to excite the DUT, hence increased robustness and reliability.

2. The value of the capacitance was picked such that it would pass the excitation pulse without distortion, while block any offset voltage, reducing power loss.

In addition, a reverse biased diode (D) was added across the output terminals to limit the residual voltage developed across the DUT (the micro-device). The schematic of the setup is shown in Figure 5.26. Not shown in this schematic are the two syringe pumps (Cat. $780100 \mathrm{C}$, Cole-Parmer, USA) that were used to introduce (or to remove) buffer fluids in the DUT through fluidic ports. An optical microscope connected with a CCD camera was used to capture sequence of pictures at every 5 seconds interval. 


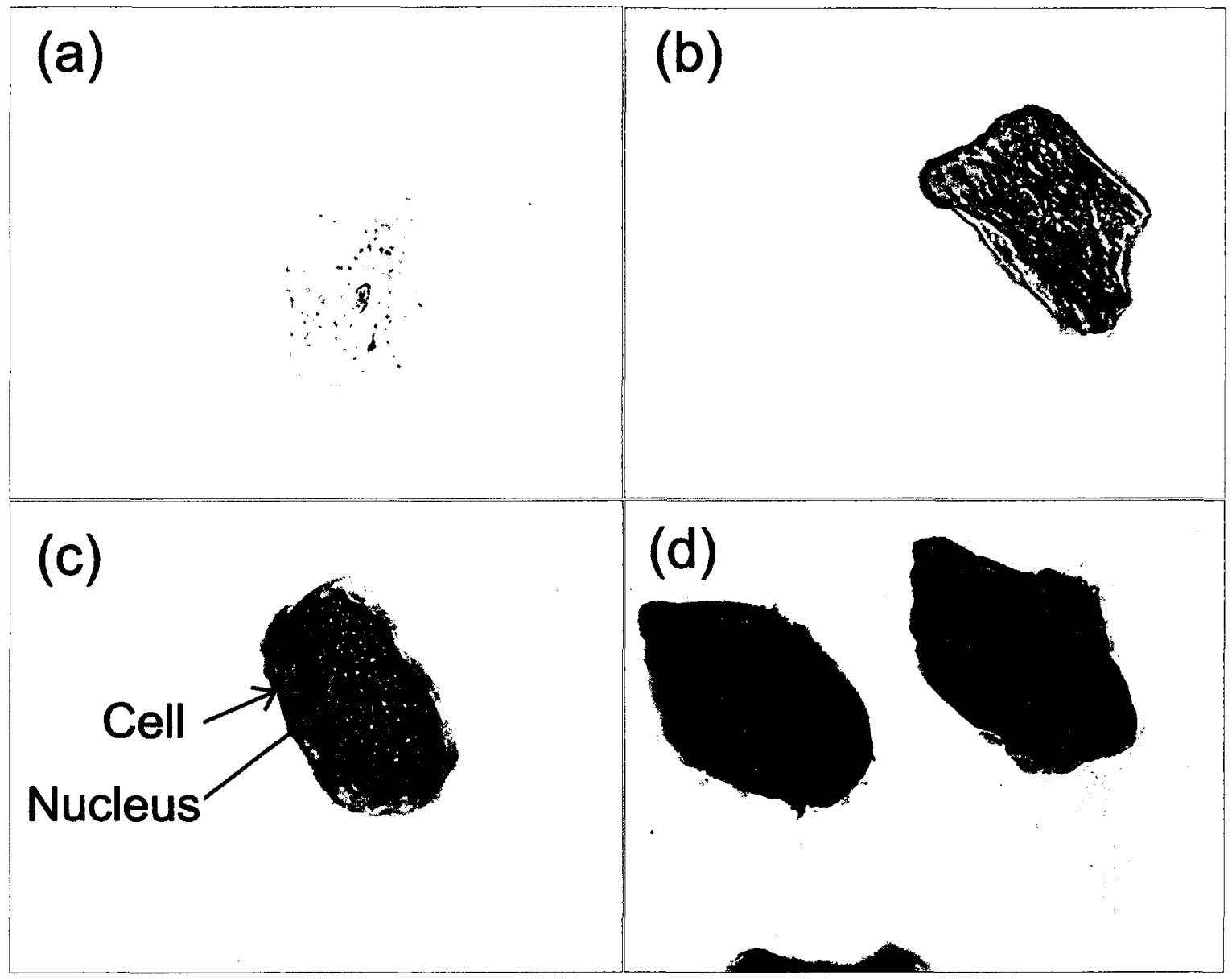

Figure 5.25 Images of cheek cells: (a) an unstained cell; (b) cell stained with Eosin stain only (that colors cytoplasm in pink/red color); (c) cell stained with both Eosin and Haematoxylin stains, where Haematoxylin stain colored chromatin (strands of DNA packed in nucleus) in blue color; and (d) stained cells observed after 1 week of storage. All images are taken with optical microscope in bright field mode with $40 \mathrm{X}$ objective lens.

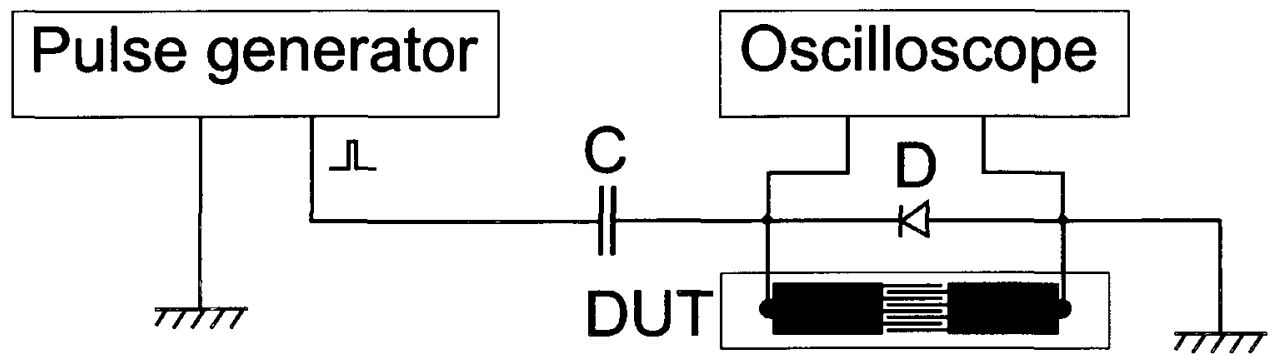

Figure 5.26 Schematic diagram of experimental setup of the device. The device under test (DUT) is connected to the pulse generator and an oscilloscope through a capacitor (C) and a diode (D). 


\subsubsection{Experimental procedure}

The experimental procedure of the device to induce EP or EL of the sample cells is given briefly here. Prior to an experiment, the micro-device is washed with flowing D- $\mathrm{H}_{2} \mathrm{O}$ thoroughly and then dried with blowing hot air. The device is then secured on the stage of a microscope so that the cell lysis chamber is in the vicinity of the focus area of the microscope. Fluidic ports are connected to capillary tubes and electrodes are connected to a pulsed power supply. The excitation source is set to deliver a number of pulses of certain duration and amplitude (depending on the requirement for $\mathrm{EP}$ or EL). The microfluidic pump injects $10 \mu \mathrm{l}$ of buffer fluids inside the microchannels (cell lysis chamber) of the micro-device through the capillary tubes and the fluidic ports at the rate of $50 \mu \mathrm{l} / \mathrm{min}$. After the buffer is loaded, a $5 \mu \mathrm{l}$ of the sample solution is introduced in the cell lysis chamber through the cell loading (i.e. sampling) ports by using a micropipette. The microscope is focused on some of the stained cells inside the cell lysis chamber. The excitation circuit is kept on hold and the image capture sequence through the CCD camera is initiated. A trigger for the pulse excitation is applied. Electrical lysis occurs as the pulse is applied throughout the cell lysis chamber. The micro-device is left undisturbed until capturing of image sequences finish. At the end of the experiment, the resultant fluid is collected using a micropipette.

\subsubsection{Image processing}

During the EP experiments, series of images were captured for visualization of stain reduction inside the sample cells. The captured images were processed later with code written in Visual Basic (VB) and Matlab. The extracted data were plotted and compared to determine the rate of stain reduction and to estimate the degree of EP. This section provides the image processing mechanism and method of implementation. 


\section{Mechanism}

The sequence of images captured during the experiments were saved in uncompressed Audio video interleave (AVI) format. For processing of each pixel data, lossy compression can not be used. The only uncompressed format available for saving the sequence of images in the image capturing software (Image-pro express) was AVI. Hence this format was used.

For processing of captured images, all individual frames were extracted from the AVI file and saved in uncompressed Bitmap (BMP) file format. Again, the reason for choosing uncompressed BMP file format is that in this format of images, each pixel information are saved separately. This eliminates possibility of loss of pixel color information due to lossy compression mechanisms. The BMP format also reduces calculation complexity of pixel color information, as all pixel data are independent of each other.

The color depth for the BMP file format can be from 1-bit to 32-bits per pixel. For our case, the color depth was chosen was 24-bits per pixel. 24-bit color depth is called "Truecolor" or "millions of colors", as it can produce over 16.7 million distinct colors. No color information is required to be saved at this or higher bit depth. Among the 24-bits, each 8-bits are used to represent red $(R)$, green $(G)$ and blue (B) color information. The value of each color can range from 0 to 255 (i.e. $2^{8}$ combinations). Each pixel is represented by a combination of these $R, G, B$ values, denoted as (R,G,B) or simply RGB. A cube representing all possible combinations of $(\mathrm{R}, \mathrm{G}, \mathrm{B})$ values is called a "color cube", as shown in Figure 5.27. The stain color of Eosin is within the region of interest as shown in the figure. The mechanism described below extract all pixels that reside within this range.

For BMP file format, information of an image is saved in several lines of rows. Each row are packed with pixel color information along one line across the width of the image. The size of each row is determined by the following expression.

$$
\text { Row size }=4 \times\left\lfloor\frac{(24 \times \text { width })+31}{32}\right\rfloor \text { bytes }
$$




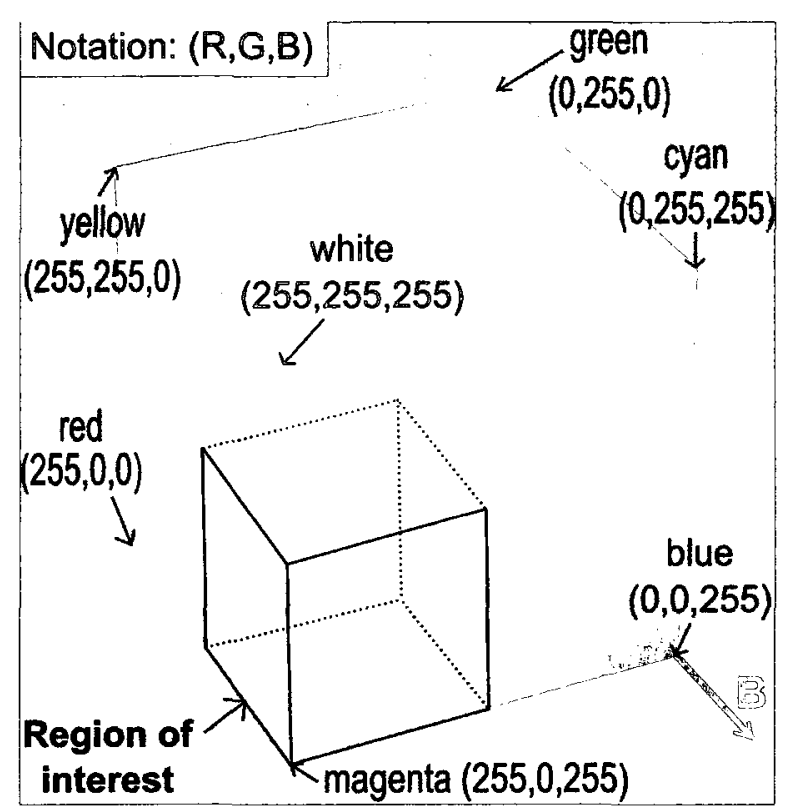

Figunre B.27 RGB color cube with red $(\mathrm{R})$, green $(\mathrm{G})$ and blue $(\mathrm{B})$ axis shown. The hidden corner at $(0,0,0)$ is black color. The region of interest (Eosin stain color) is indicated.

Here $\lfloor\cdot\rfloor$ represents the floor function. Individual rows contain pixel information of lines along the height of the image. For example, if an image is 800 pixel wide and 600 pixel high, the color information will be saved in 600 rows, where each row will be 2400 bytes long. In addition, each file will have standard header at the beginning that specifies file type related information.

The concept of this image processing mechanism for this research is to plot the number of pixels in each row that reside within the range of interest. This is demonstrated using a simplified case as shown in Figure 5.28. A $5 \times 5$ pixel image is considered for ease of explanation. In the first row of the image, there is no pixel with $R G B$ value within the range of interest. Hence the corresponding data in plot shows 0 as the number of stained pixels. The next row contains 1 pixel within the range of interest and correspondingly shown as 1 in the plot. The third row contains 2 of the pixels within the range of interest. So the number of stained pixels for this row is 2. The next row contains 4 pixels within the range and the last one contains 1 pixel. Thus the number of pixel counts are plotted along y-axis (right side of the 


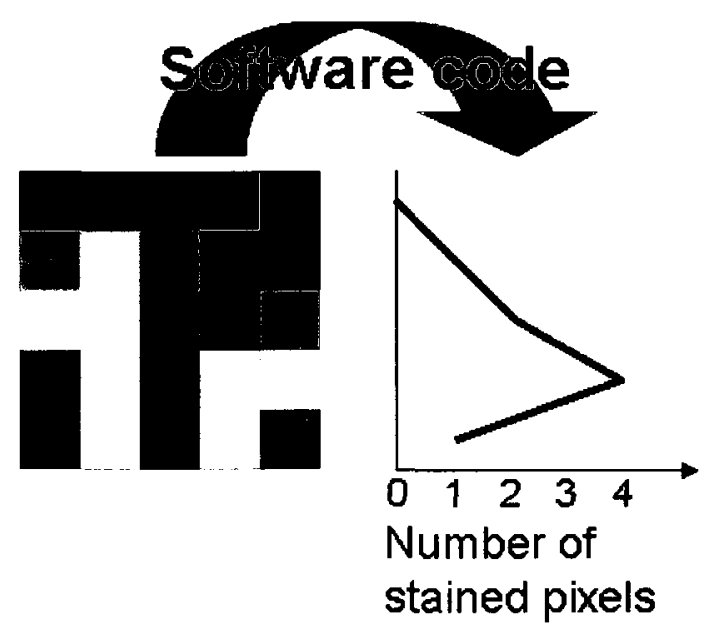

Figure 5.28 A sample showing the extraction of pixel data from an image into a plot. Here a $5 \times 5$ pixel image is illustrated to simplify the concept. Each pixel within the region of interest increments the count for that row (assuming y-axis plot is intended). Total count of the stained pixels from each row is plotted against the row (horizontally aligned, but not marked) on the right side as a line graph.

figure), where all these data in the plot are joined using a line graph. Using the same technique, scanning along the columns alow plotting along $\mathrm{x}$-axis.

This type of plot allows quantification of image data and comparison of stains among various images of a sequence. In addition, stain reduction rate also becomes comparable. Thus this image processing enabled us to deduce various conclusions after the images are processed, as discussed below.

\section{Program description}

To extract the number of pixels within the range of interest, a program has to scan each row and determine whether individual pixels are inside or outside of the range of interest. If a pixel is inside the range of interest, a count value is incremented. After the complete row is scanned, the count value is stored corresponding to that row. After all rows of the image is scanned and a total count value is calculated by adding all count values of various rows.

Direct scanning of the BMP files for determination of RGB range is not possible as 

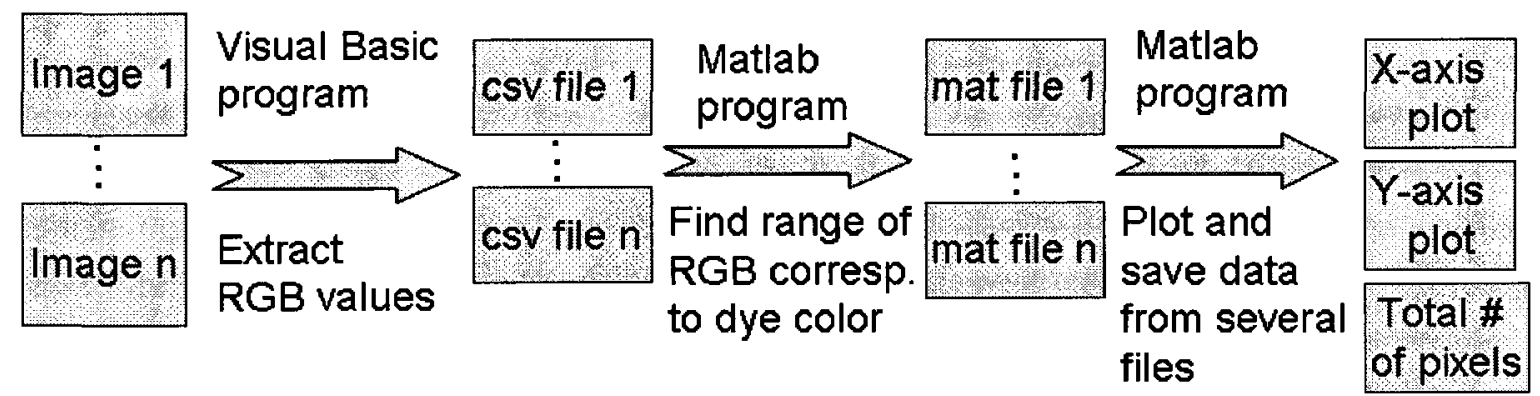

Figure 5.29 Procedure involved in extraction of pixel counts and plotting the corresponding data. The VB code sequentially processes all images of the captured sequence and stores data in CSV file format. A Matlab code analyzes the data of CSV files and determines the pixel counts for each row. Another Matlab code plots this extracted data and stores the total count of stained pixels.

the data in BMP files are saved in byte-format (machine code), rather then American Standard Code for Information Interchange (ASCII) format. The latter format is required for mathematical computation (such as comparison). So the first step of the process is to convert all pixel data from the byte-format to ASCII format. This step was achieved through a VB code, that scans all image files of a sequence sequentially and saves the corresponding data of each pixel in ASCII format in individual files in Comma separated values (CSV) file format.

Next, all CSV files of a sequence of images are read by a Matlab code that compares the RGB value of each pixel and determines whether the pixel color is inside or outside of the range of interest. A count value is increased if the RGB value is within the range of interest. The count values from all rows of an image are saved in a MAT (Matlab data) file. Another Matlab code loads one or more MAT data files and plots the corresponding result. If more than one file is loaded, different colors are used for data from various files with lagend. The code also saves the total number of count value of each MAT file by adding all count values from each row of the image file. The complete procedure is represented in Figure 5.29.

Here the described procedure is specific for plotting along $\mathrm{y}$-axis, which was primarily used in this study. However, the Matlab codes developed are also capable of 

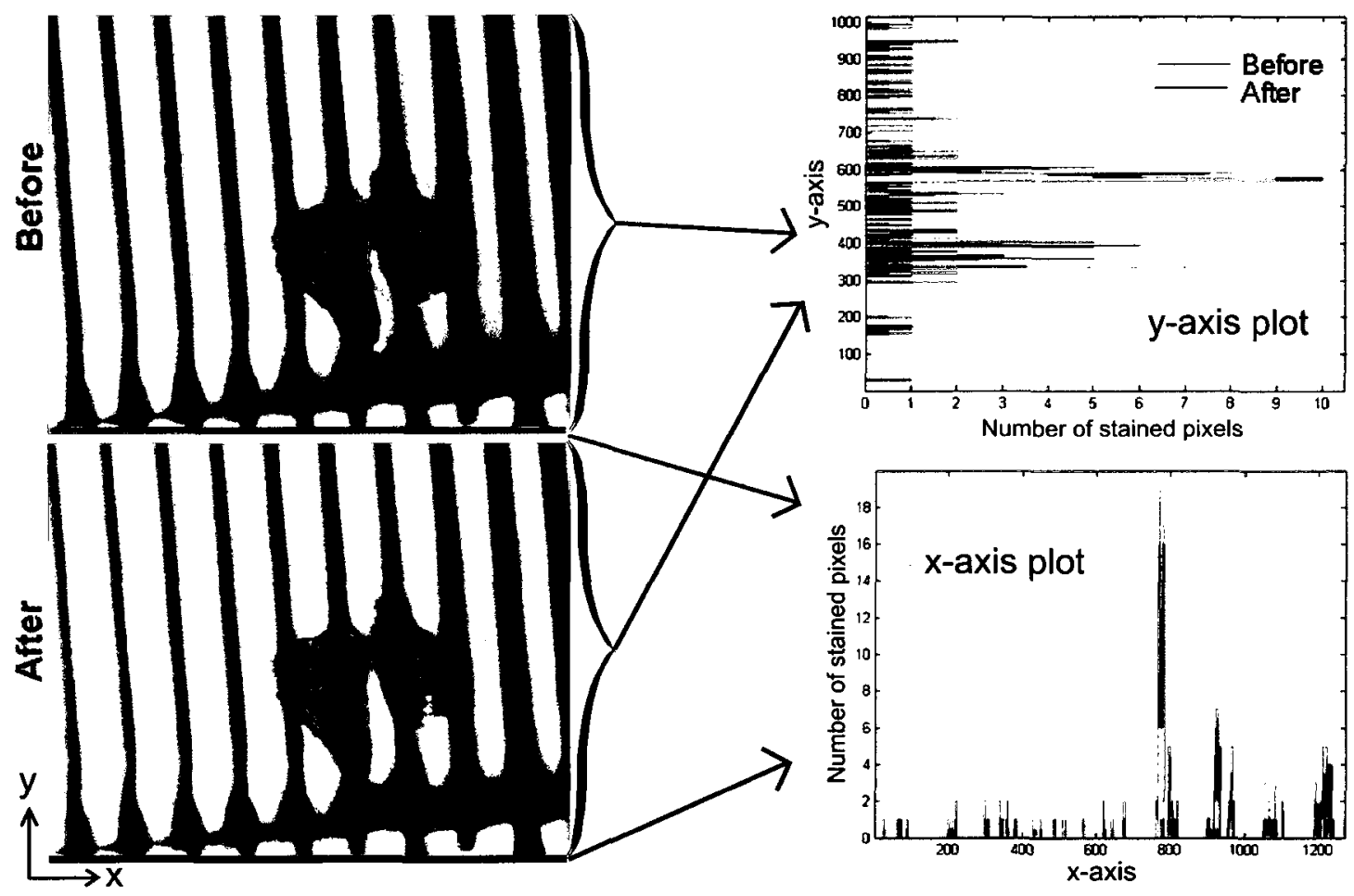

Figure 5.30 Extraction of pixel data from a set of images during an EP experiment on the left side and corresponding pixel data plot in overlapped form on the right side. Data was plotted along both $\mathrm{x}$ and $\mathrm{y}$-axis of the images. The images and the plots are not aligned.

plotting along $\mathrm{x}$-axis. In the latter case, column data of the CSV file are scanned instead of row data, and the rest of the procedure is similar.

\section{Validation}

Some sample results from the image processing technique developed in this study are illustrated here to validate the image processing software coded developed.

In the first example (Figure 5.30), two images were processed using the developed programs. The images on the left side were captured from EP experiments. The dark thick lines along $y$-axis of these images on the left side were the electrodes, which were $20 \mu \mathrm{m}$ apart from each other. Two stained cells were visible in each of these images. The cells were inside a microchannel of the device. The top image on the left 


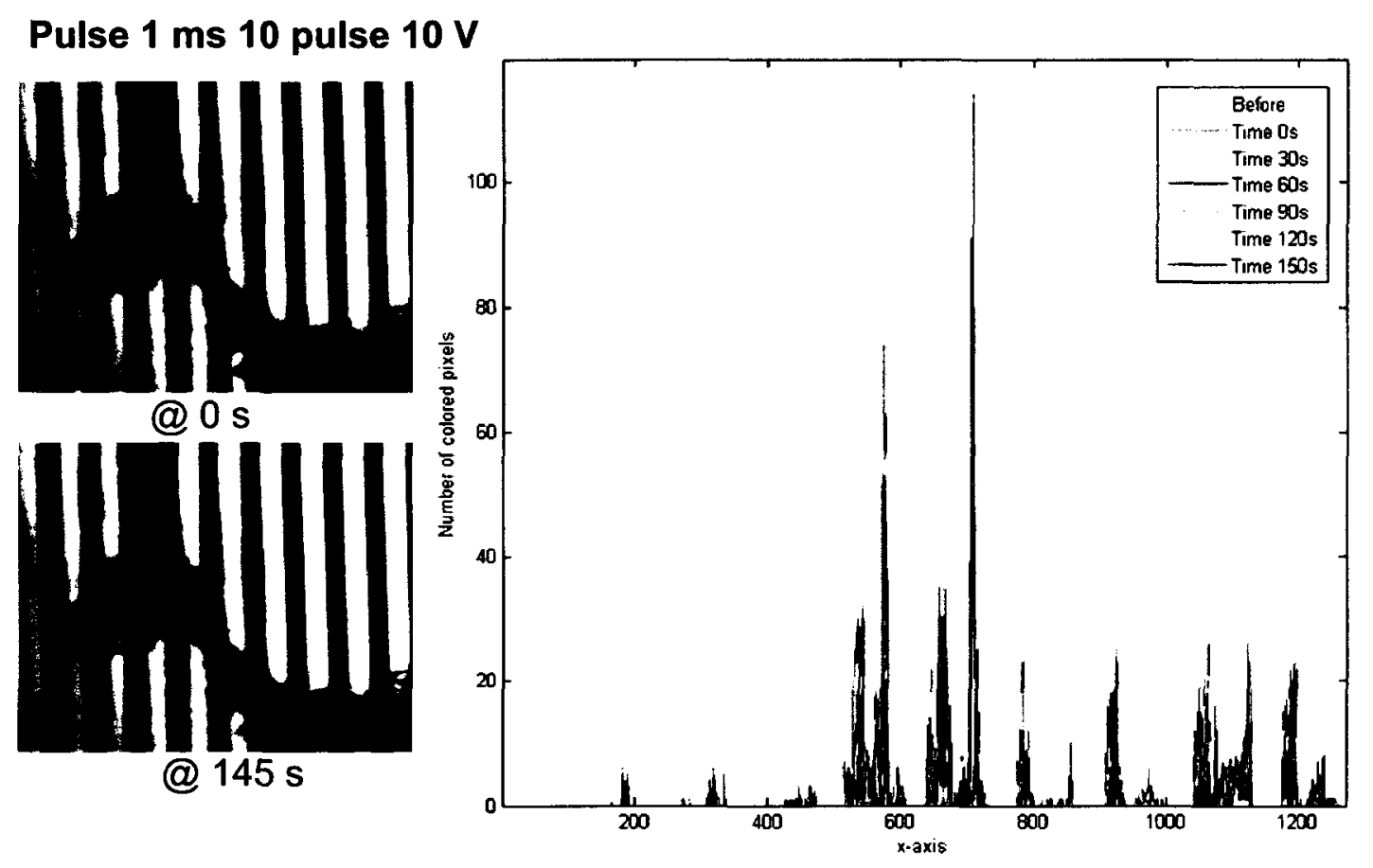

Figure 5.31 Images captured during an EP experiment are shown on the left side. Time series plot of the number of stains along y-axis are depicted on the right side.

side was captured just prior to the application of an excitation pulse. The bottom image on the left side was captured 5 seconds after the excitation pulse.

The extracted data were plotted on the right side for both $\mathrm{x}$ and $\mathrm{y}$-axis. Data from the top left side picture (Before) was plotted in green color, whereas data from the bottom left side picture (After) was plotted in red color. Note that the pixel count peaks where the pink stained cell is located along both axes, not where the blue stained cell. Also some ambient color are detected as stain in case of green plot (noise error). In the $\mathrm{x}$-axis scan plot, no stains were detected where electrodes are located. Generally, y-axis plot was preferred for this analysis.

Figure 5.31 and Figure 5.32 demonstrates time series overlapped plots of 7 images along $\mathrm{y}$-axis and $\mathrm{x}$-axis, respectively. Two stained cells were observed in each image. The vertical dark bars were the electrodes and the circular shape on the top right side 
Pulse $1 \mathrm{~ms} 10$ pulse $10 \mathrm{~V}$
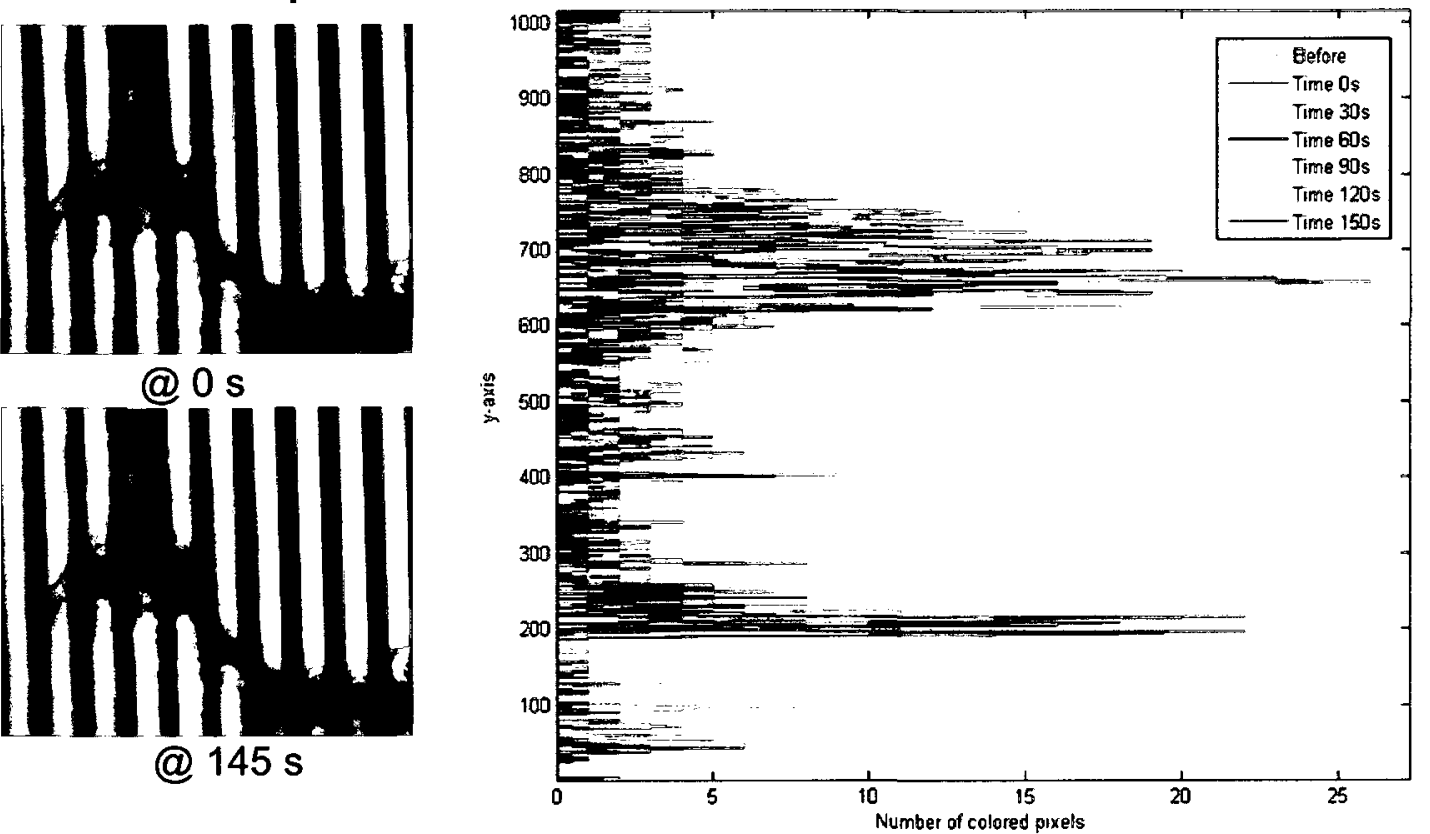

Figure 5.32 Images captured during an EP experiment are shown on the left side. Time series plot of the number of stains along $\mathrm{x}$-axis are depicted on the right side. 
were the cell sample loading port. The excitation pulse was applied right after the first image of the sequence. The images processed for this plot were captured every 30 seconds during an EP experiment.

Extracted data using the VB and Matlab codes were plotted on the right side in both figures. The pixel count on the average was observed to decrease over time. Similar to the previous example, $\mathrm{x}$-axis plots shows no data where the electrodes were located.

As discussed before, the Matlab code stores the total number of pixels saved in each image. A plot of this total number of images showed an exponentially decreasing rate for stained pixel reduction. To be able to compare among various excitation parameters, image sequences obtained from different experiments were processed to obtain Normalized Stain Reduction (NSR) plots. The normalized value of the total stained pixels of each image is calculated by dividing the total count of stained pixels of an image by the total count of stained pixels of the first image of that sequence. The resultant metric is denoted as NSR that removes dependency on the coverage of stained pixels in an image.

A time series plot of NSR is shown by the red dots in Figure 5.33. The image also shows the mathematical formula for calculating NSR. The excitation pulse was applied right after the capturing of the first image of the sequence. The decrease rate of NSR was well matched to an exponential curve shown as green dashed line in the figure. Corresponding expression for $y$ and $R^{2}$ were extracted using Excel, as shown in the figure. The value of $R^{2}$ is close to 1 that indicates good match of the experimental data with the fitted curve. Thus the image processing allowed us for various quantitative analysis from experimental data captured through sequence of images. 


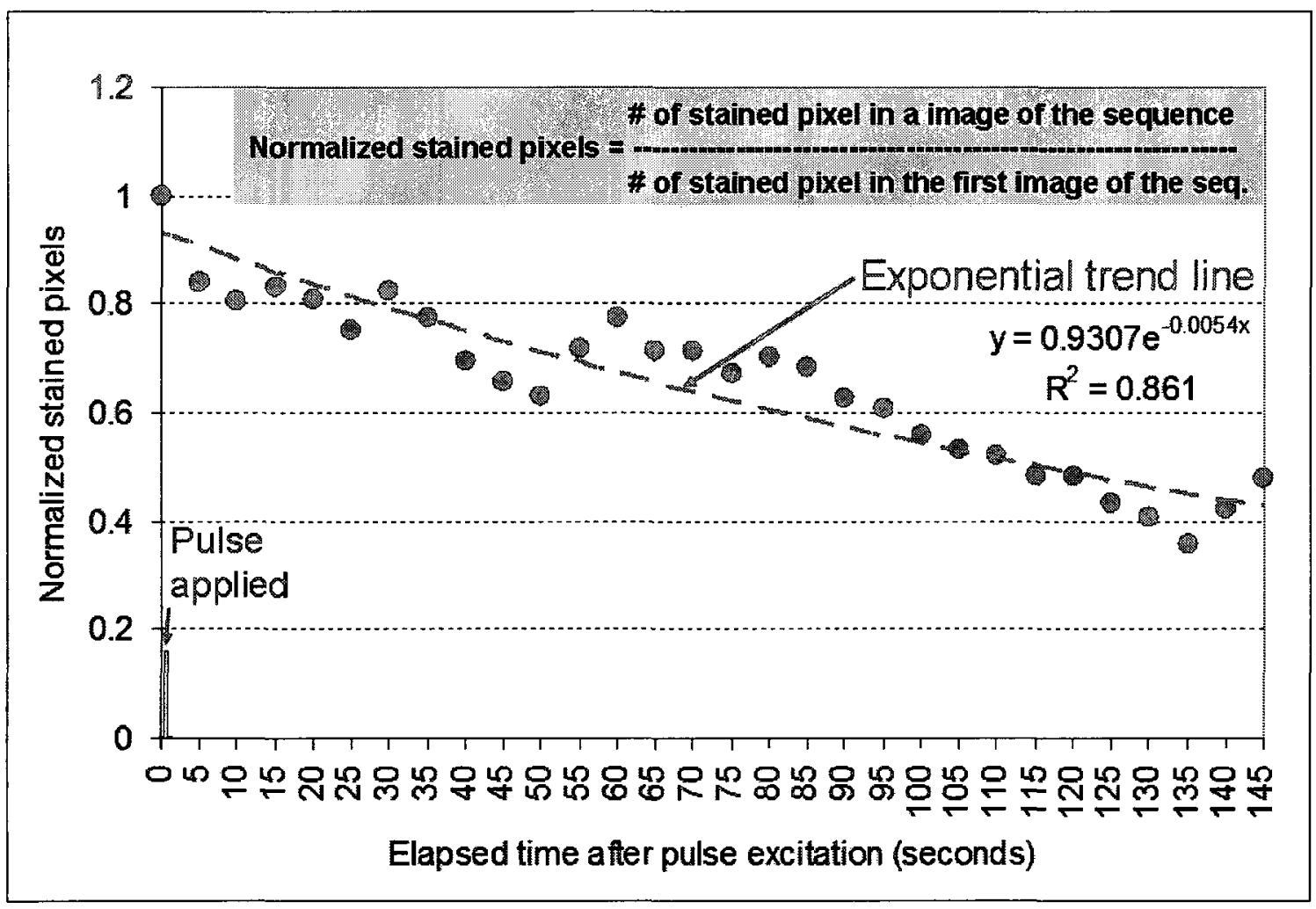

Figure 5.33 Exponential decrease of Normalized Stain Reduction (NSR) of pixels over elapsed time. Data were extracted from 30 images of a sequence over 2.5 minutes duration. Total number of pixel counts were used for this plot. 


\subsubsection{Electroporation experiments}

Stained cells in D- $\mathrm{H}_{2} \mathrm{O}$ does not lose stains (Hematoxylin or Eosin) with time, even after a week of storage (Figure 5.25). The $\mathrm{pH}$ of $\mathrm{D}-\mathrm{H}_{2} \mathrm{O}$ is about 7.0 that keeps the cell membrane stable and intact [75]. However, applying pulses of sufficient strength can create pores in cell membrane to cause the stains to dilute (either by fluids exiting the cell and reducing stains within cells, or surrounding fluid entering the cell and reducing stain concentration in cells). As the process of EP makes the cell membrane permeable, this allows degradation of Eosin stains as extracellular fluid enters or intracellular fluid exits cells. Note that the Hematoxylin stain should not change concentration as basophilic materials (such as chromatin or DNA) cannot transport through these reversible pores formed during EP.

Note that the gap between pulses, when a pulse train was applied, was equal to the pulse width, if not indicated otherwise.

Experimentation of EP with non-invasively collected cheek cell samples (collected using buccal swabs) revealed that the Eosin stain degrades significantly with application of pulses. In Figure 5.34, two picture sets from EP experiments are presented. The top picture set represents control experiment (Case 1), where no excitation was applied. Stains of cell did not degrade in this case. The bottom picture set represents a pulse excitation experiment (Case 2), where a pulse of $10 \mathrm{~ms}$ duration and $20 \mathrm{~V}$ magnitude was applied at time 0 . In this latter case, Eosin stain reduced significantly as observed in the picture taken after $2 \mathrm{~min}(60 \mathrm{~s})$ of pulse excitation.

Case 2 of Figure 5.34 is elaborated in Figure 5.35, where the temporal sequence of pictures (image sequence) taken every $5 \mathrm{~s}$ from the application of the pulse is given. Note that cells move freely inside the microchannels of the cell lysis chamber. The figure also shows that bubbles are formed as soon as pulse is applied (due to Faraday's reaction at Cathode and Anode terminals of the micro-device). The reduction of stain can only be explained with EP concept, which states that applying electric field of sufficient strength induces hydrophilic pores within the cell membrane. Also note 

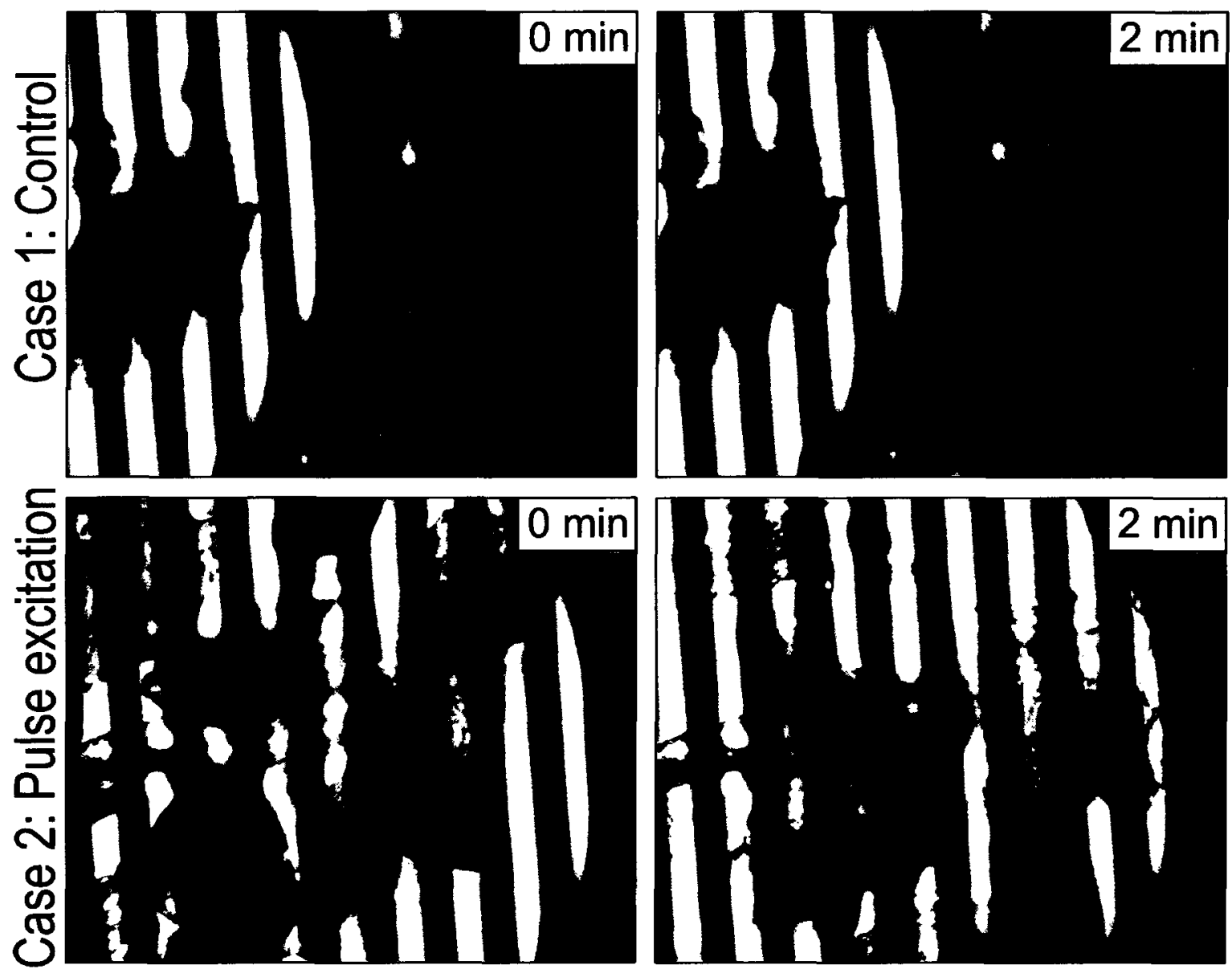

Figure 5.34 Two image sets (captured with 20X lens using the CCD camera attached with optical microscope) from EP experiments with $\mathrm{D}-\mathrm{H}_{2} \mathrm{O}$ as buffer medium. In the top set of pictures (Case 1: Control), stained cells retained colors (stains) after 2 minutes. In the bottom set of pictures (Case 2: Pulse excitation), Eosin stains reduced significantly when a pulse of $100 \mathrm{~ms}$ with magnitude of $20 \mathrm{~V}$ across the electrodes was applied. 

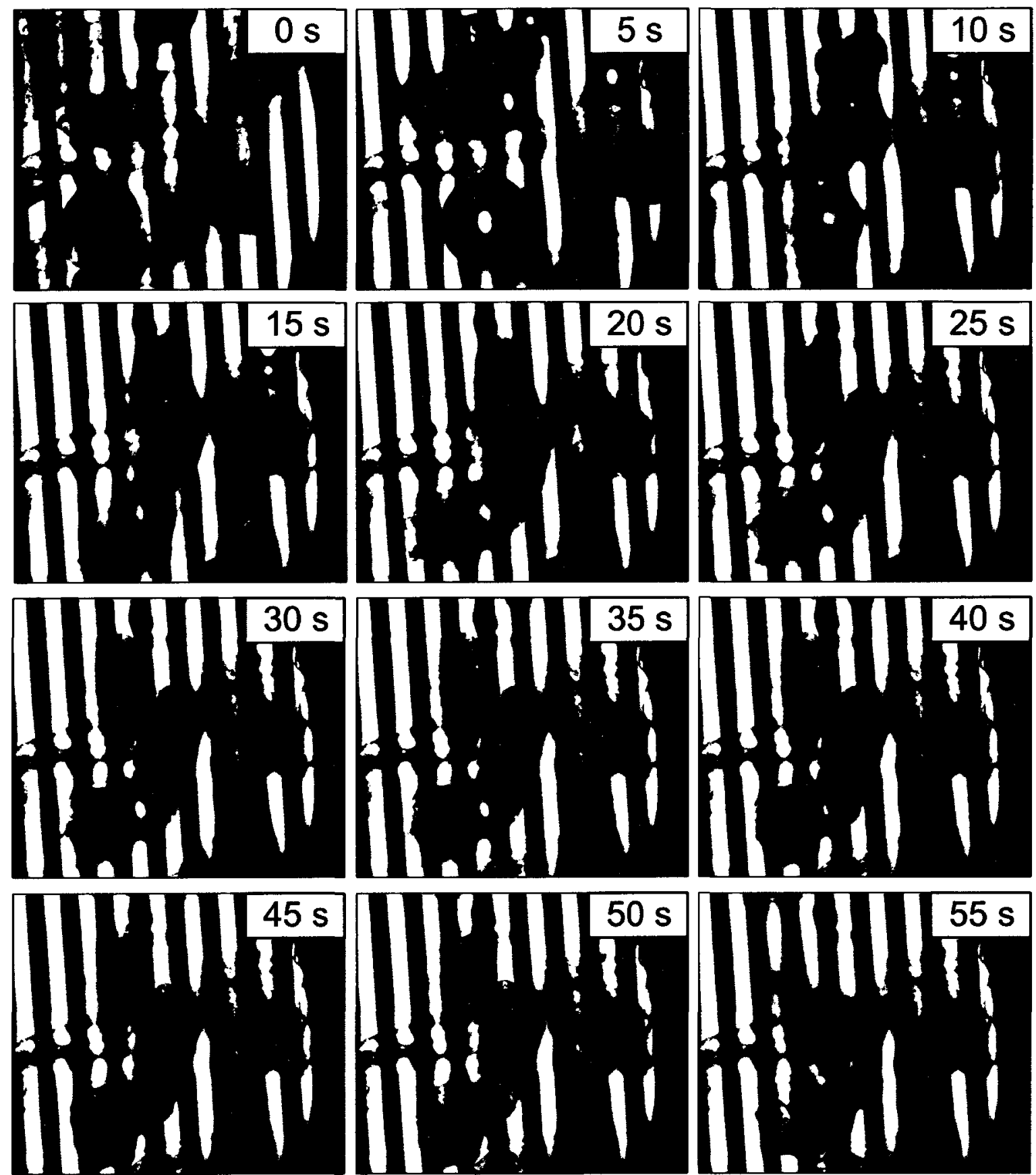

Figure 5.35 Sequence of pictures obtained from the CCD camera showing temporal changes as a $20 \mathrm{~V}$ pulse of $100 \mathrm{~ms}$ duration is applied across the electrodes at $0 \mathrm{~s}$ (Case 2 of Figure 5.34). Here stained cells were suspended on $\mathrm{D}-\mathrm{H}_{2} \mathrm{O}$ medium. 
that the morphological deformation in shapes of the cells in Figure 5.35. Most of the cells have reduced diameter, that suggest that fluids from inside the cell (stained cytoplasm) exited through cell boundary causing cells to shrink.

To quantify the stain reduction, we have utilized image processing technique as described earlier in this section. Briefly, the stain color information were extracted from each image using a Visual Basic program and analyzed data using a Matlab program (Appendix Section C.4). The program determined number of pixels within the range of interest (Figure 5.27), i.e. stain color of Eosin, in each image of the sequence. This data is used to calculate NSR, defined as the ratio of (number of stained pixel in an image within the sequence) over (number of stained pixel in the first image of that sequence), as noted in Figure 5.33. The advantage of using NSR over absolute value of stained pixels is that the NSR metric eliminates dependency on actual number of stained pixels within the image taken (as different experiments might have different number of cells within the viewing area), thus making extracted data comparable among different experimental cases.

We have conducted a number of experiments over a range of applied voltage (from $2.5 \mathrm{~V}$ to $20 \mathrm{~V}$ ), pulse width (from $1 \mu \mathrm{s}$ to $5 \mathrm{~s}$ ) and number of pulses (from 1 to 100) to determine the pulse parameters that cause EP (as well as EL, as discussed in the next section). A summary of experimental observations are given in Table 5.3.

The observation of EP was conducted by the image analysis previously discussed to find NSR at $145 \mathrm{~s}$ after excitation (30 frames at interval of $5 \mathrm{~s}$ ). If the change of NSR at $145 \mathrm{~s}$ was less than $5 \%$, the the excitation was considered to cause "No Effect". If the reduction of stain was higher, but cell membrane was not disintegrated or damaged (visually detectable), then the excitation was considered to cause EP. Finally, if the cell membrane were broken or severely damaged through large holes, the excitation was considered to cause EL. Each experimental condition was conducted as least 3 times (i.e. number of samples, $\mathrm{N}=3$ or more).

Noting that the number of pulses multiplied by pulse width can be expressed as total time of excitation, hence the experimental conditions can be plotted in a two- 
Table 5.3 Summary of experimental parameters and corresponding observation using the final device

\begin{tabular}{|c|c|c|c|}
\hline Applied potential (V) & Pulse width (s) & Number of pulses & Observation \\
\hline 2.5 & $1 \times 10^{-3}$ & 10 & No effect observed \\
\hline 2.5 & $1 \times 10^{-6}$ & 10 & No effect observed \\
\hline 5 & $1 \times 10^{-6}$ & 1 & No effect observed \\
\hline 5 & $1 \times 10^{-6}$ & 10 & No effect observed \\
\hline 5 & $100 \times 10^{-6}$ & 1 & No effect observed \\
\hline 5 & $10 \times 10^{-6}$ & 100 & No effect observed \\
\hline 5 & $1 \times 10^{-3}$ & 1 & EP observed \\
\hline 5 & $1 \times 10^{-3}$ & 10 & EP observed \\
\hline 5 & $1 \times 10^{-3}$ & 25 & EP observed \\
\hline 10 & $10 \times 10^{-6}$ & 1 & No effect observed \\
\hline 10 & $100 \times 10^{-6}$ & 1 & No effect observed \\
\hline 10 & $1 \times 10^{-3}$ & 10 & EP observed \\
\hline 10 & $100 \times 10^{-3}$ & 1 & EP observed \\
\hline 15 & $1 \times 10^{-3}$ & 10 & EP observed \\
\hline 15 & $100 \times 10^{-3}$ & 1 & EP observed \\
\hline 20 & $1 \times 10^{-6}$ & 1 & No effect observed \\
\hline 20 & $1 \times 10^{-6}$ & 2 & No effect observed \\
\hline 20 & $1 \times 10^{-6}$ & 10 & No effect observed \\
\hline 20 & $10 \times 10^{-6}$ & 1 & EP observed \\
\hline 20 & $10 \times 10^{-8}$ & 10 & EP observed \\
\hline 20 & $10 \times 10^{-6}$ & 100 & EP observed \\
\hline 20 & $1 \times 10^{-3}$ & 1 & EP observed \\
\hline 20 & $1 \times 10^{-3}$ & 10 & EP observed \\
\hline 20 & $10 \times 10^{-3}$ & 1 & EP observed \\
\hline 20 & $10 \times 10^{-3}$ & 10 & EP observed \\
\hline 20 & $20 \times 10^{-3}$ & 1 & EP observed \\
\hline 20 & $100 \times 10^{-3}$ & 1 & EP observed \\
\hline 20 & $500 \times 10^{-3}$ & 1 & EL observed \\
\hline 20 & 1 & 1 & EL observed \\
\hline 20 & 5 & 1 & EL observed \\
\hline
\end{tabular}


dimensional space with one axis as the applied potential and the other axis as the total time of excitation. The experimental results are plotted in such as plot space as given in Figure 5.36. Note that such a plot space provides information on the energy being delivered through the excitation, as energy can be expressed in terms of applied voltage and total time of excitation. The bottom left hand corner represents the lowest energy region, whereas the top right hand corner represents the highest energy region. From the plot, it is evident that we can separate the total plot space in three distinct zone or regions: (i) EL region on the top right hand corner, where energy is highest, (ii) EL region in the middle, where energy is moderate, and (iii) the region where no observable effect were noted from experiments, where energy is lowest. Such observation is consistent with previous reports [112]. However we have noted that some overlapping points of EP and no-effect regions. After analysis of a cell model using the proposed pore model in the current cell model as discussed in the next chapter, the reasoning for the presence of such overlapping points can be rationalized.

Various excitation conditions produced different levels of stain reduction during EP. To compare various cases of EP, we have compiled 3 plots with the following constrains:

1) Effects of number of pulses on NSR for an applied voltage of $5 \mathrm{~V}$ and pulse duration of $1 \mathrm{~ms}$ (Figure 5.37).

2) Effects of various excitation pulse parameters for an applied voltage of $20 \mathrm{~V}$ (Figure 5.38).

3) Effects of applied voltage for a fixed pulse parameter of 1 pulse of $10 \mathrm{~ms}$ duration (Figure 5.39).

From Figure 5.37, increasing number of pulses increase the stain reduction with the same applied potential and pulse width. Increased number of pulses with certain pulse witth and amplitude deliver increased amount of energy. This moves the experimental condition upwards in Figure 5.36 towards higher energy region. A higher energy is likely to induce more pored during EP, resulting higher reduction of stain within a 


\section{EP and EL observations over various excitation conditions}

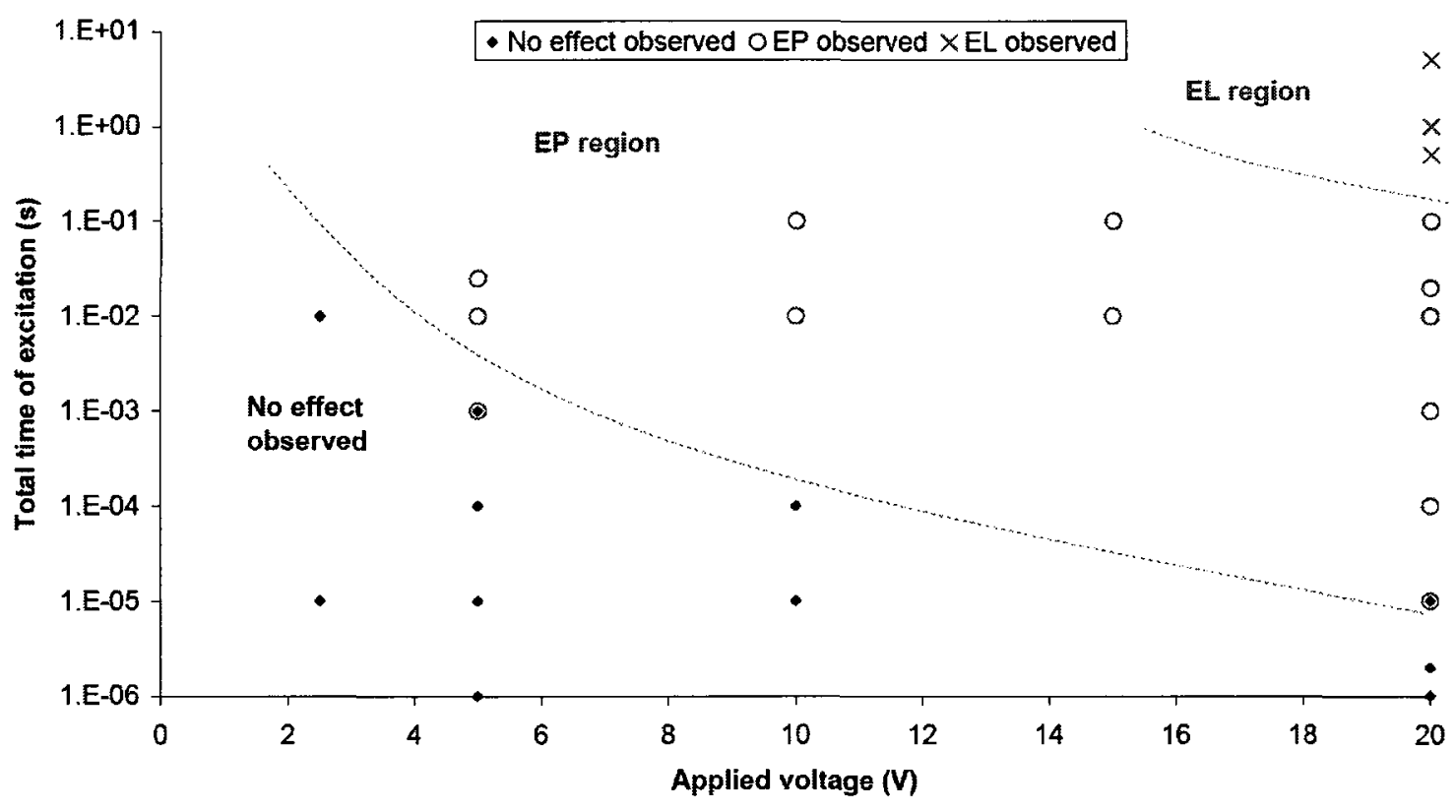

Figure 5.36 Combined plot of EP and EL related result showing various excitation conditions and revenant observations. The resultant plot can be divided into 3 regions: No effect observed, EP region and EL region. 


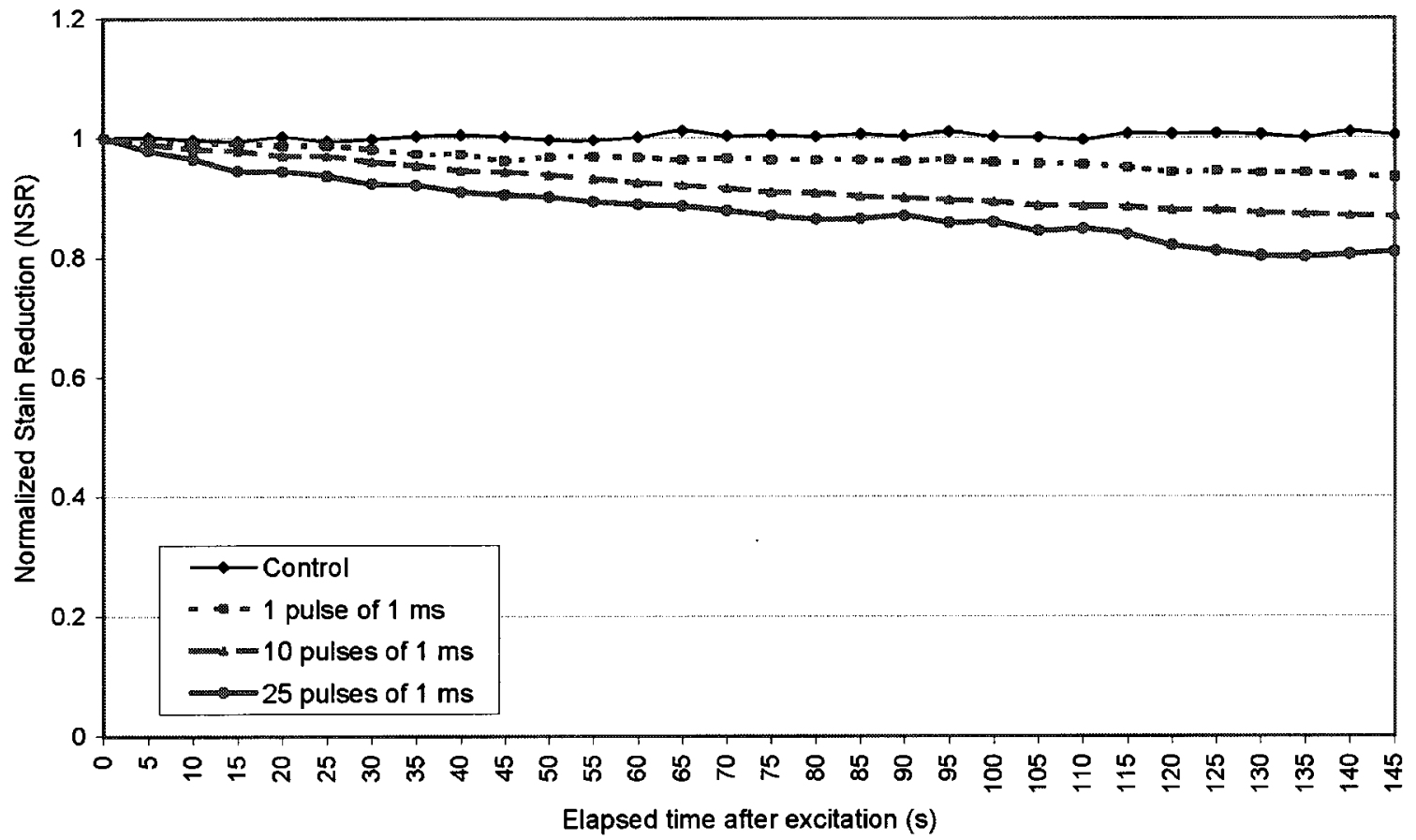

Figure 5.37 Normalized stain reduction with time for various excitation condition with $5 \mathrm{~V}$ of applied potential. The NSR plots are averaged over 3 samples. The control case is not excited by any electrical pulse. 


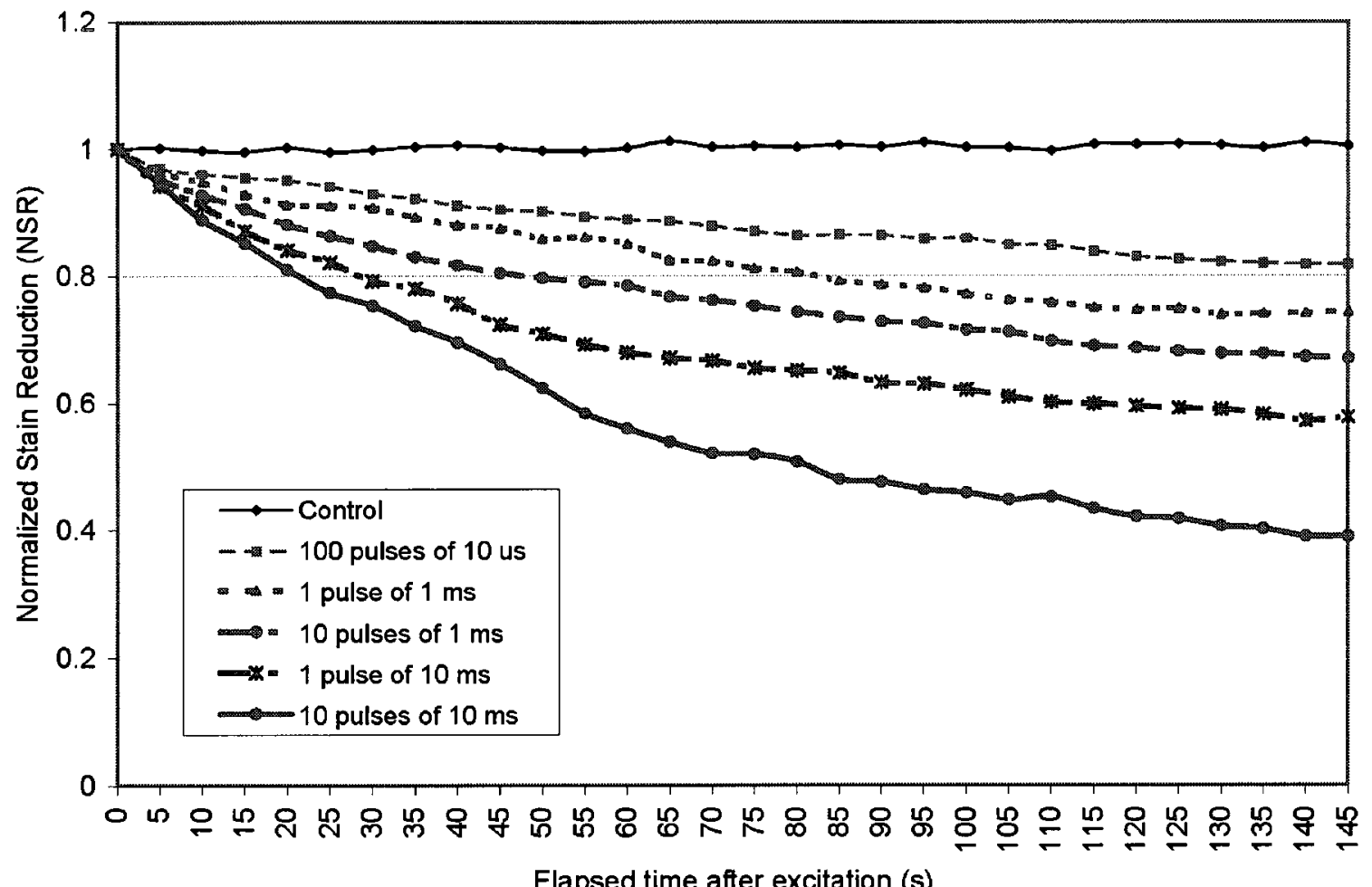

Elapsed time after excitation (s)

Figure 5.38 Normalized stain reduction with time for various excitation condition with $20 \mathrm{~V}$ of applied potential. The NSR plots are averaged over 3 samples. The control case is not excited by any electrical pulse. 


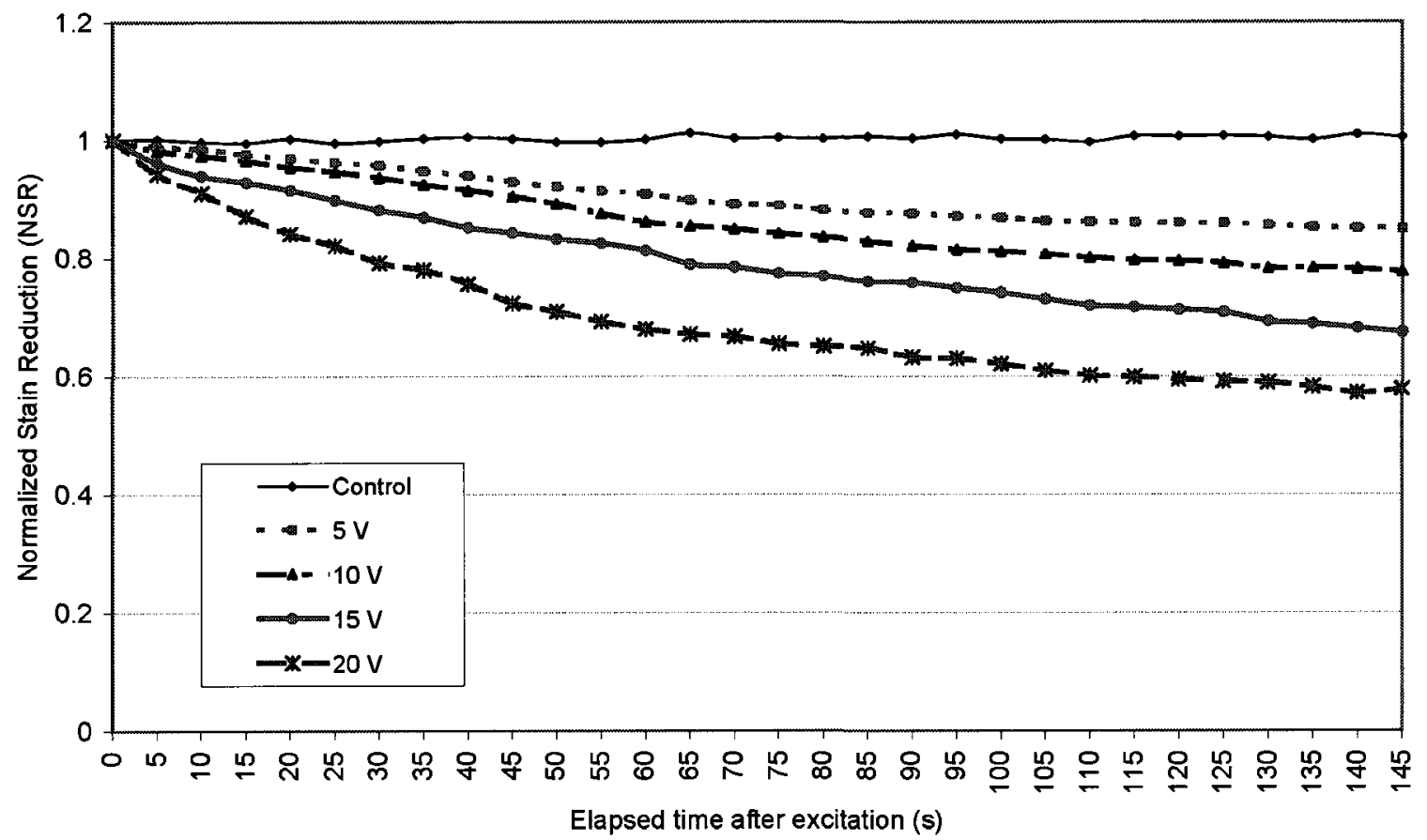

Figure 5.39 Normalized stain reduction with time for various excitation voltage with the same pulse parameters: 1 pulse of $10 \mathrm{~ms}$ duration. The NSR plots are averaged over 5 samples. The control case is not excited by any electrical pulse. 
certain time period.

Figure 5.37 shows a region on the far right hand side of EP region of Figure 5.36 where the applied potential is $20 \mathrm{~V}$. As in last figure, here also note that 10 pules of 1 ms duration causes more reduction of stains compared to 1 pulse of $1 \mathrm{~ms}$ duration or 10 pulses of $10 \mathrm{~ms}$ duration causes more reduction of stains compared to 1 pulse of 10 ms duration. However, 100 pulses of $10 \mu$ s duration results less reduction of stained pixels compared to 1 pulse of $1 \mathrm{~ms}$ duration, even though in both cases, the total time

of excitation is $1 \mathrm{~ms}$ (i.e. the energy delivered is constant). Hence, a single pulse can induce more reduction of stain compared to multiple pulse when delivering a fixed amount of energy. The same can be made for 10 pulses of $1 \mathrm{~ms}$ duration compared to 1 pulse of $10 \mathrm{~ms}$ duration. Note that for fixed amount of energy delivery using a certain applied potential, the excitation point in the plot space of Figure 5.36 does not change.

Figure 5.37 shows the effect of pulse amplitude on the number of stained pixel reduction for a pulse of $10 \mathrm{~ms}$ duration. As the applied potential is increased, increase amount of stain reduction occurs for the same pulse parameters. In terms of the experiment location within the plot space of 5.36, higher applied potential moves the experimental point towards the right where energy is higher.

\subsubsection{Electrical lysis experiments}

As electrical lysis requires high energy pulses with magnitude high enough to create above threshold electric field, all EL results were observed on the upper right hand side of the plot space in Figure 5.36. Such pulses however cause significant amount of electrolysis resulting large number of bubble formation. Observation of longer pulses ( $0.5 \mathrm{~s}$ or longer) were hindered by these formation of numerous bubbles that obstructed observation (as with MetalMUMPs device discussed earlier in this chapter). In this section, we have presented significant results with EL experiments, progressively describing effects of larger pulse widths. 
With larger pulses (larger than $100 \mathrm{~ms}$ ), cell membrane structures were seen to be broken or destroyed (Figure 5.40). In this figure, prior to pulse application (a), many visible cell structured were noted that were stained with pink/redish Eosin stain. However, right after the application of the pulse (b), few cell structures can be seen. In addition, the increase pink/red color in buffer medium suggest that the cytoplasm is diffused in the medium. This is possible when cell lysis occurs. Hence the minimum excitation pulse that were required for EL with our final micro-device was $20 \mathrm{~V} 0.5 \mathrm{~s}$ pulse. However, this pulse does not guarantee lysis of all cells within cell lysis chamber, as few cell structures are still visible after the excitation (Figure $5.40 \mathrm{~b})$. Note that with this pulse condition, still the number of bubbles generated are not many and observation during pulse excitation was possible.

With increased pulse duration of $1 \mathrm{~s} \mathrm{(20} \mathrm{V} \mathrm{excitation),} \mathrm{cell} \mathrm{structures} \mathrm{were} \mathrm{seen}$ to be completely destroyed (Figure 5.41). The H\&E stained cells were not observed in images after excitation, even after the disappearance of bubbles formed during the excitation. Note the center of the loading port where observation is not hindered by bubble formation as shown in Figure 5.41(c). Here the image of Figure 5.41(b) is subtracted from the image of Figure 5.41(a) to show the relative position of visible area in Figure 5.41(b).

For complete lysis of all cells in cell lysis chamber, as well as to release DNA from nucleus with the cells, a single pulse of $20 \mathrm{~V}$ amplitude and $5 \mathrm{~s}$ duration was used (Figure 5.42). The dissipated power for this condition was $0.4 \pm 0.1 \mathrm{~W}$, calculated using the voltage drop of $1 \mathrm{~V}$ across the supply unit with internal resistance of 50 ohms. The energy spent with these pulse parameters were $2 \pm 0.5 \mathrm{~J}$. Note that to release DNA from inside of cells, nuclear membrane must also be ruptured as well as cell membrane. Nuclear membrane is similar structure to cell membrane, however has two layers of membrane. In addition, protein content in nuclear membrane are much less compared to cell membrane.

Figure 5.42 provides two images of the same horizontal location of the microdevice, but at two different vertical height. Figure 5.42(a) is focused near the bottom 

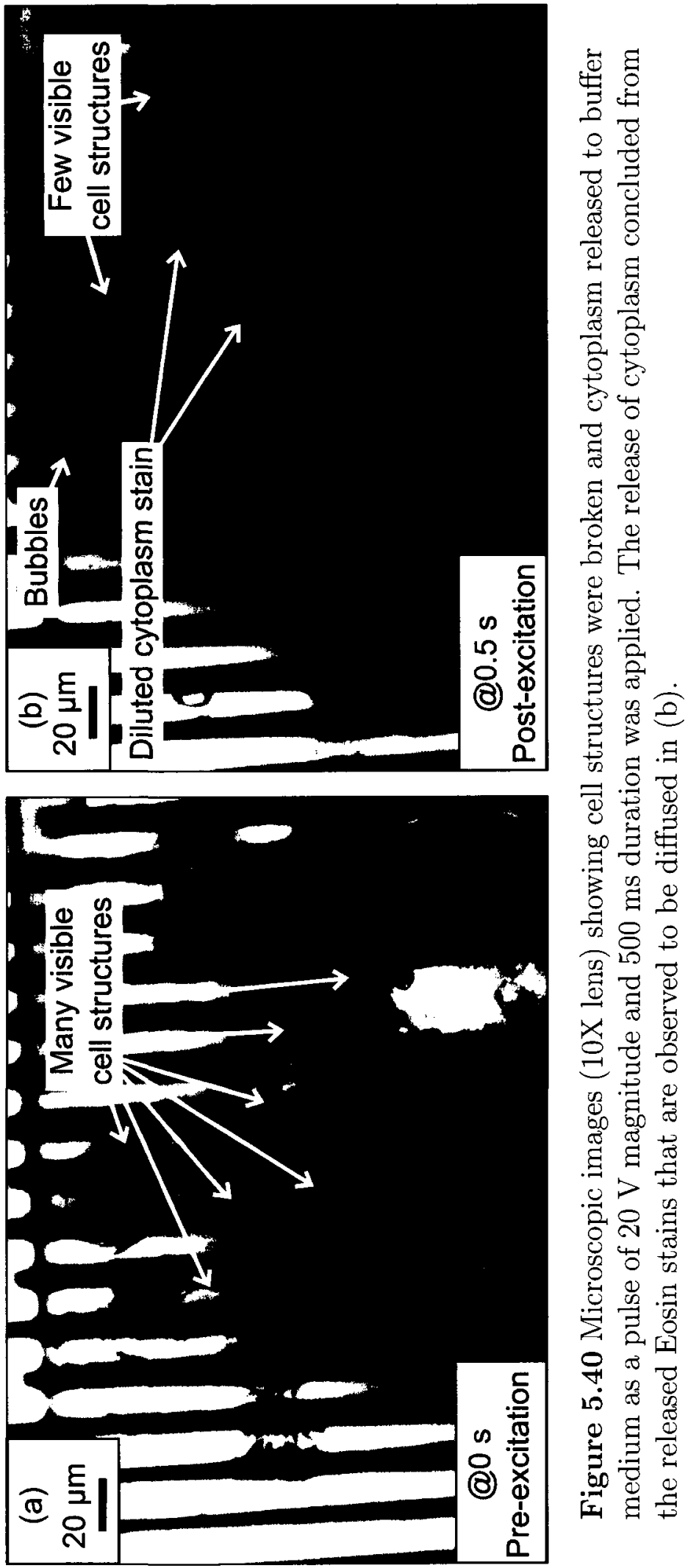
$=$ 응 응

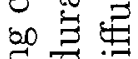

च

官 \&

용

क 8

过

○。

क :

范

ฮี

द्व छ

$\circ>$

융요

谟需

进

$\sum_{\text {五。 }}$

구 $\sigma$

1. क

¿

舟导 


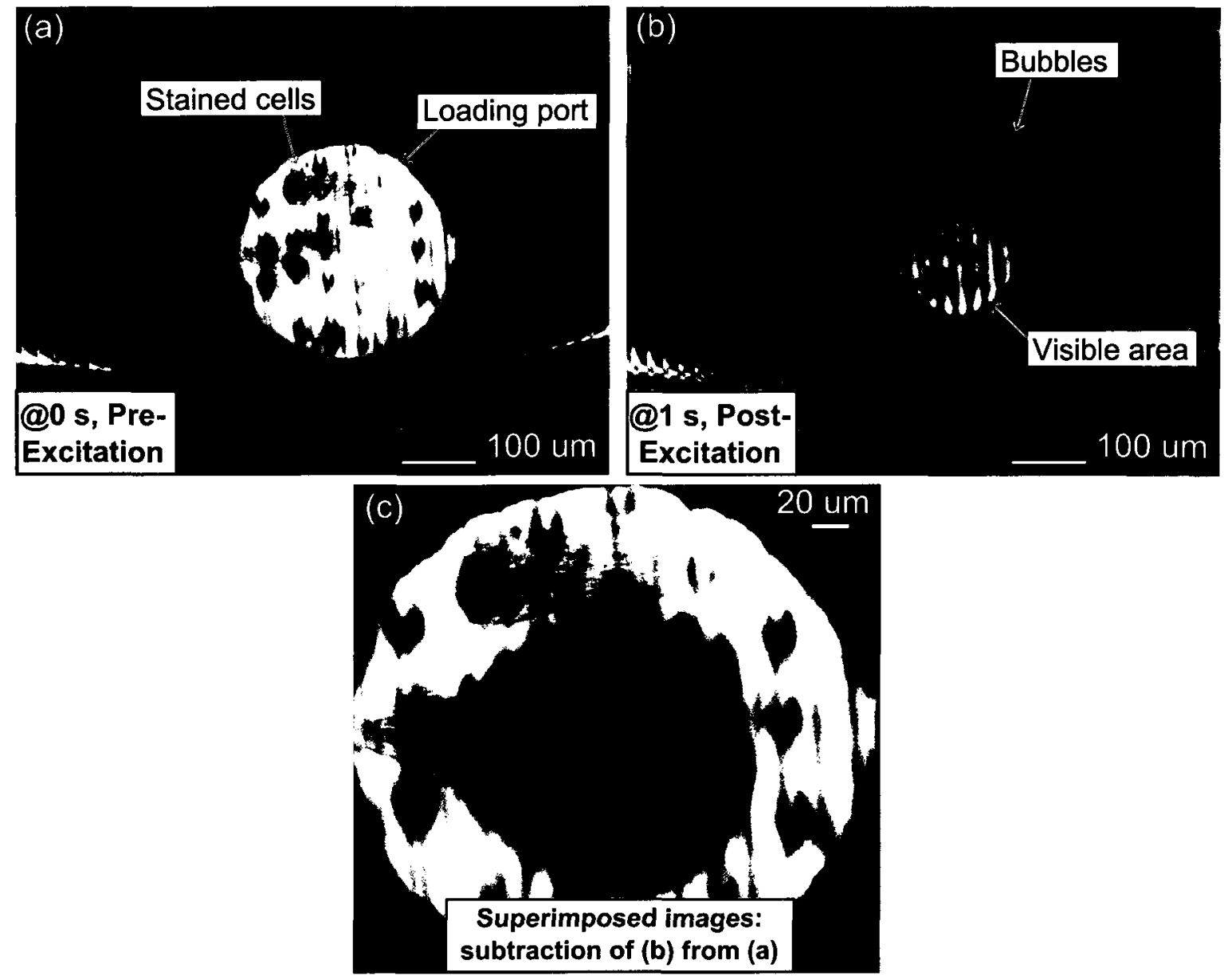

Figure 5.41 Observation under a microscope (10X magnification) after applying a single pulse of $20 \mathrm{~V}$ magnitude and $1 \mathrm{~s}$ duration. (a) Image prior to excitation (at $0 \mathrm{~s}$ ). (b) Image immediately after excitation (at $1 \mathrm{~s}$ ). Bubbles due to excitation limited visible area. (c) Superimposed images obtained by subtracting image (b) from image (a) and enlarged to show the loading port. 
of the microchannel, whereas Figure 5.42(b) is focused about $10 \mu \mathrm{m}$ vertically above the earlier image. These images show exposed DNA (identified with blue Haematoxylin stain) outside cell structure. Moreover, de-stained cell membranes are observed on the right hand side of the images. As these cell membranes are completely destined and does not possess cell structure, it suggests that these cell membranes are sheet of cell membrane that randomly attaches with each other.

To further investigated the released DNA in Figure 5.42, the dotted area from both images are enlarged and shown in Figure 5.43. It is very interesting to note the intensity differences between these images. The image on the left hand side was at the bottom level of the microchannel, where electrodes are clearly observed (focal plane), indicated by red arrows. However on the image to the right hand side, these electrodes are not clearly observed indicating the focus plane has been changed from the bottom of the microchannel. Now, note different spots of DNA as indicated by red arrows in this image. This proves that the DNA is spatially distributed along the height of the microchannel. This also confirms the release of DNA from cells after EL using the relevant excitation condition.

Furthermore, we have analyzed Figure 5.42(a) to reduce background color using standard image processing procedure to confirm the DNA stain from the image, as shown in Figure 5.44. Note the completely distinguishable blue color of stains from DNA in the middle of the image. Also note that the cell membranes on the right side does not contain any color similar to these, suggesting all DNA is released outside the cell membrane. Judging from the size (area) of DNA stain, it is larger than nucleus that were depicted in previous figures. Hence the DNA must have been exposed outside of nucleus, suggesting disrupters of nucleus with the specific excitation.

The released cell membrane was observable in other locations of the micro-device as shown in Figure 5.45. The resulted solution is collected from the micro-device and observed under an optical microscope with $100 \mathrm{X}$ oil-immersion microscope, as given in Figure 5.46. The DNA strands were identified by the blue Haematoxylin stain that binds to DNA and the extremely coiled shape of DNA resulted from DNA 

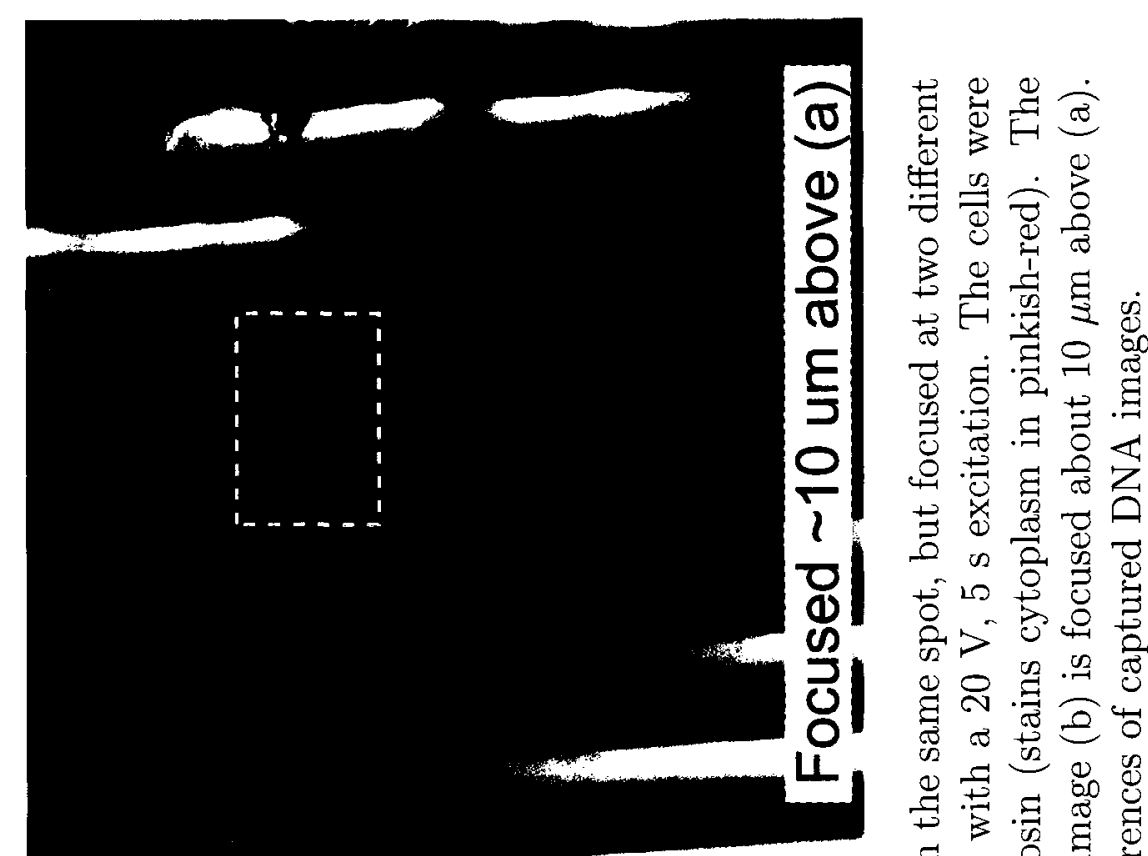

क

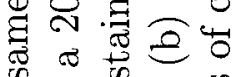

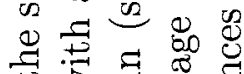

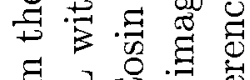

园国国吾苞

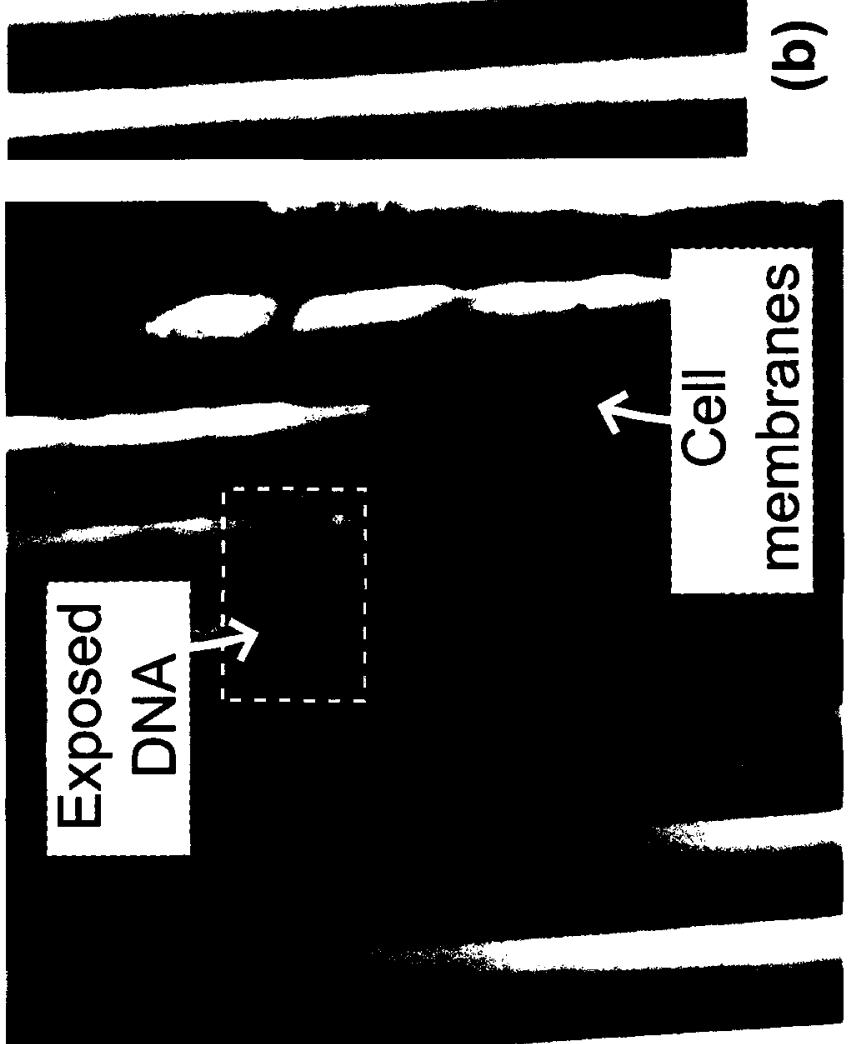

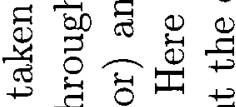

형효 형

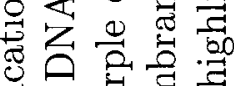

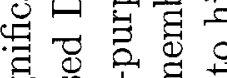

50 o d

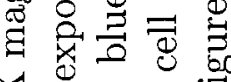

伲

焉范若离

品公落

త్ర

$. \exists . \overline{3} \cdot . \Xi$

莕票

萧《

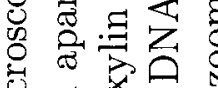

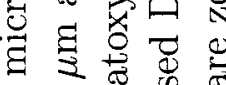

○잉 क

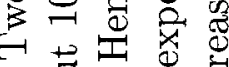

स 苛

ฟै

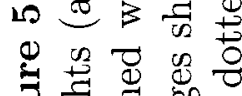

(1) 


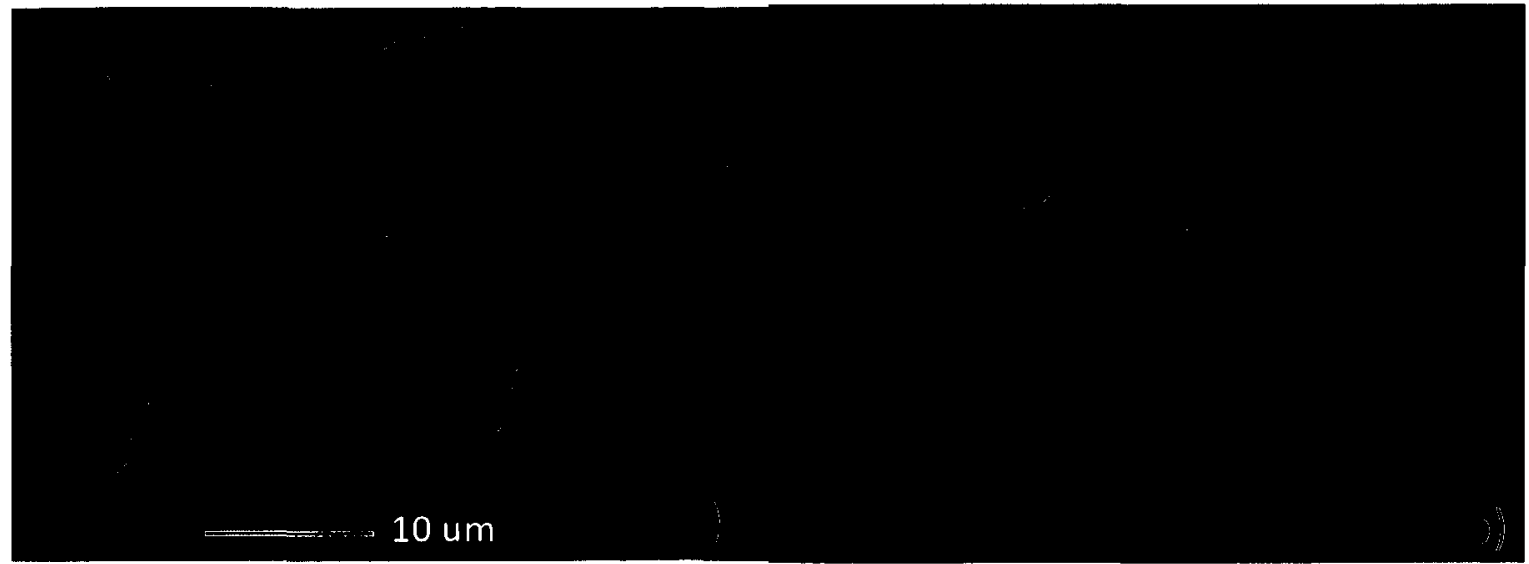

Figure 5.43 Enlarged images showing exposed DNA at two vertical heights within the micro-device. The difference of height between the images is about $10 \mu \mathrm{m}$. The arrows indicate intensity differences between the images taken from the same spot, demonstrating that DNA stands were distributed vertically in spatial domain compared to electrodes.

double helix and coiling around histone proteins inside nucleus. The average power consumption of DNA extraction with $20 \mathrm{~V} 5 \mathrm{~s}$ pulse was $0.4 \pm 0.1 \mathrm{~W}$, as recorded by the oscilloscope.

\subsubsection{Reusability of the device}

After several experiments, the electrodes degrade and defects are formed. Two types of defects, shown in Figure 5.47, were observed:

1) Crack: Some integrated interdigitated electrodes inside the microchannels developed thin cracks. Such cracks disconnected a portion of the electrode from the remaining electrodes, thus not conducting current for excitation.

2) Lift off: In severe cases, portions of electrodes were completely lifted off from the device and was carried away with the flow of the buffer fluid. This also resulted open circuit of electrodes, as well as the lifted fragment of electrode might cause short circuit between other electrode pairs inside the microchannel. 


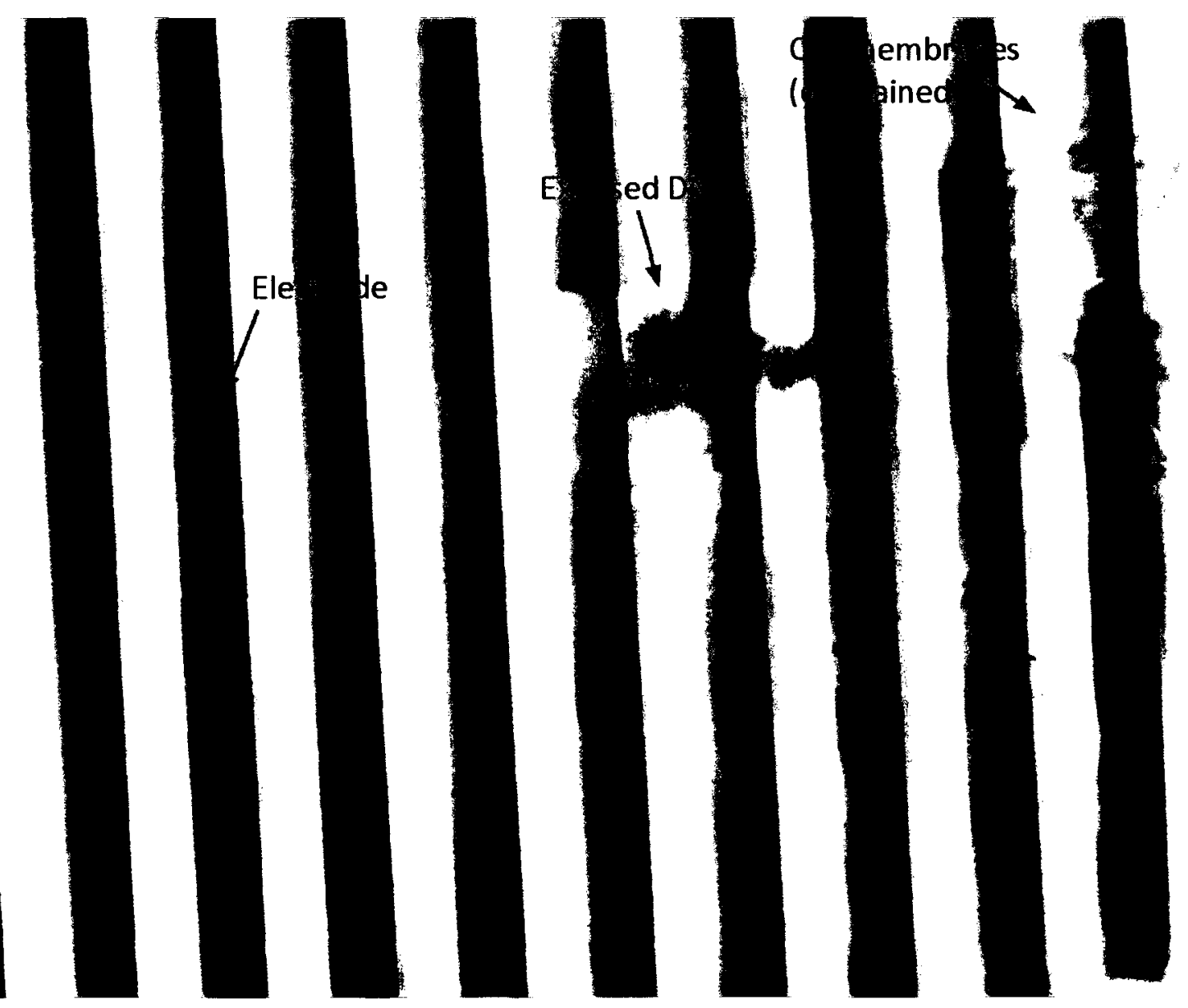

Figure 5.44 One of the images of exposed DNA is processed to reduce background color using standard image processing technique, and shows stained DNA clearly. 


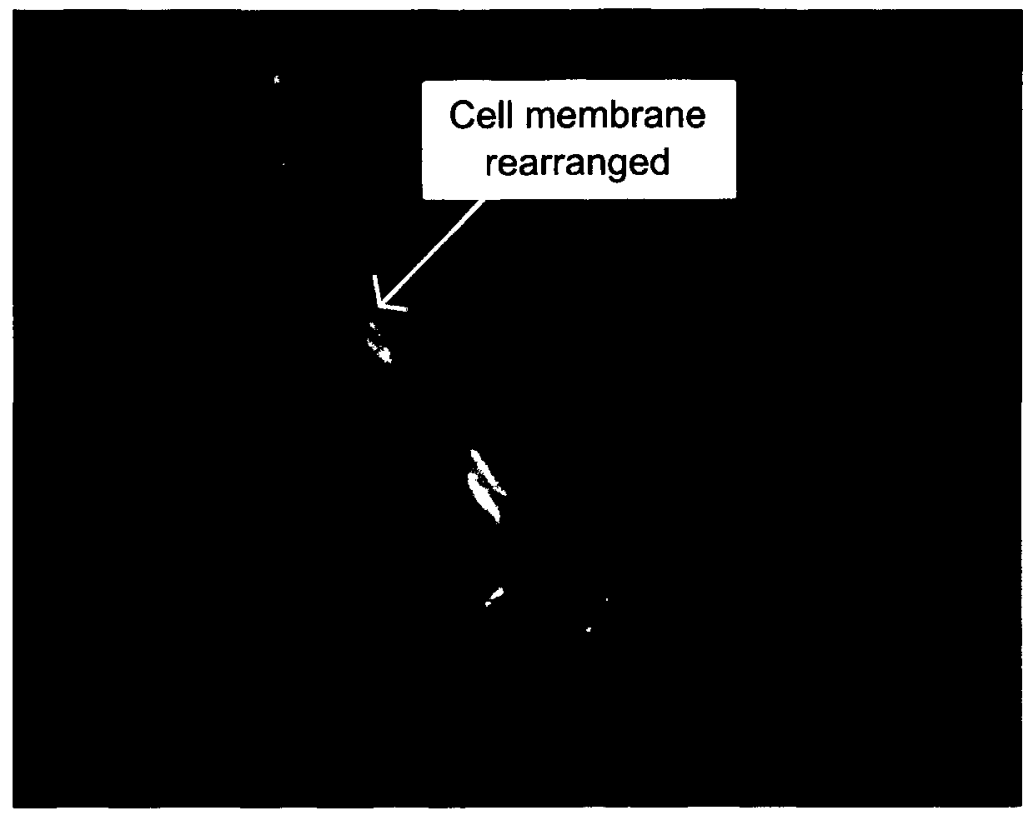

Figure 5.45 Rearranged cell membrane observed under the optical microscope (20X lens) that is apparently in complete irregular shape observed after EL experiments.

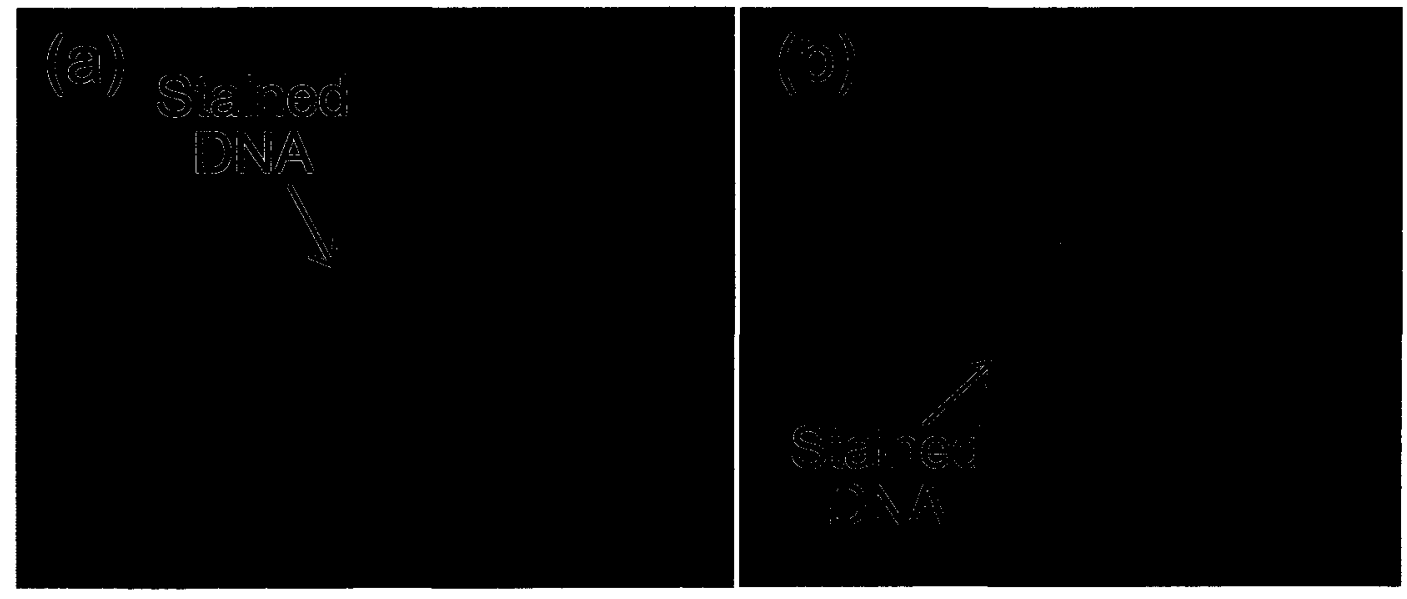

Figure 5.46 Two samples of exposed DNA observed with 100X objective lens (oil immersion) in collected fluids from the micro-device after excitation of 20 $\mathrm{V}$ for $5 \mathrm{~s}$. The DNA strands are identified by the blue stain of Haematoxylin and the coiled structure that is unique to DNA stands (chromatin). 

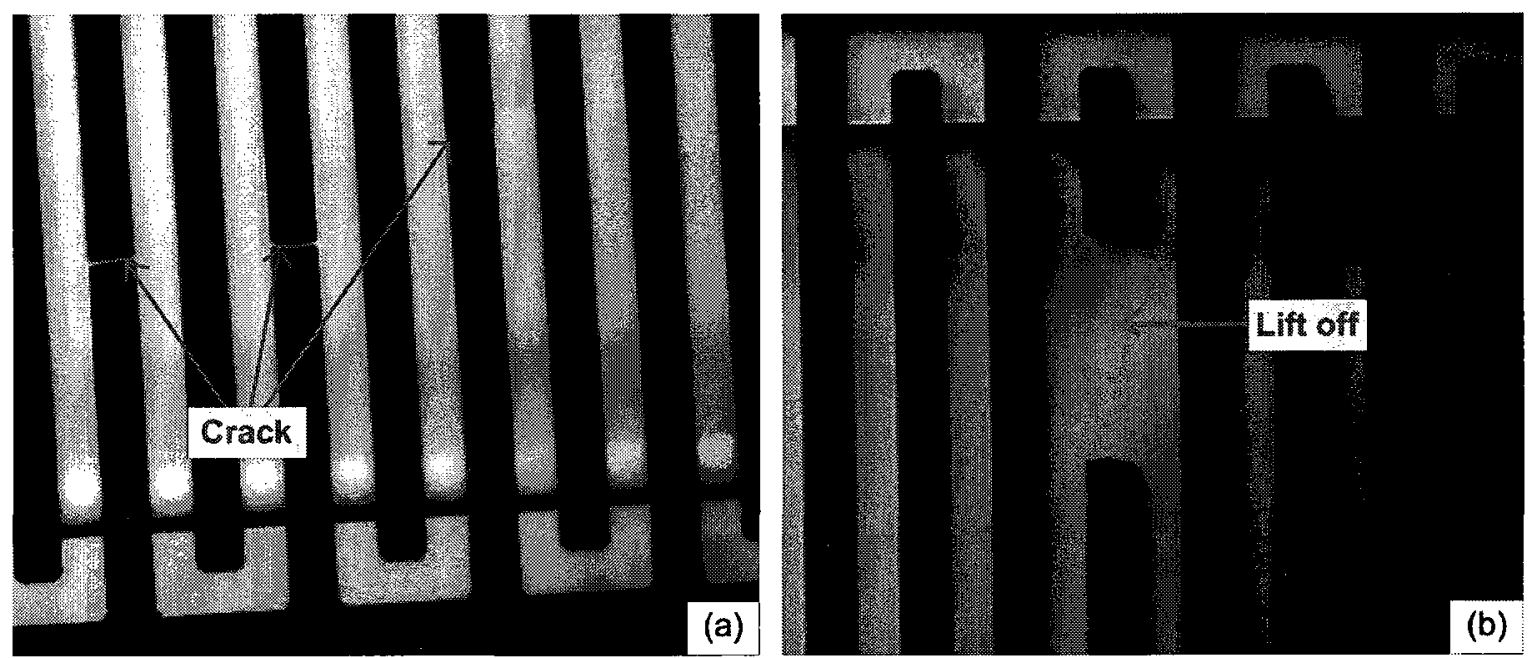

Figure 5.47 Microscopic images (20X lens) showing two types of defects developed through electrode degradation inside the device: Crack and Lift off.

It was noted that electrodes connected to anode side were only degraded (Figure 5.47), that leads us to believe that the electrochemical reaction as the possible cause of such defects, as the chemical reactions at Anodes is different that those at Cathodes. If heat was the cause of such defect, both electrodes would have been effected.

Following electrochemical reactions occurs at Anode and Cathode side of the electrodes, where in Anode side, the adhesion layer, Tantalum (Ta), would react with the ions available to form powder type Tantalum Oxide $\left(\mathrm{Ta}_{2} \mathrm{O}_{5}\right)$ :

Electrolysis of water:

$$
\begin{array}{rcc}
\text { Cathode: } & 2 \mathrm{H}_{2} \mathrm{O}+2 e^{-} & \rightarrow \mathrm{H}_{2}+2 \mathrm{OH}^{-} \\
\text {Anode: } & 4 \mathrm{OH}^{-} & \rightarrow \mathrm{O}_{2}+2 \mathrm{H}_{2} \mathrm{O}+4 e^{-}
\end{array}
$$

Dissolving of tantalum:

$$
\text { Anode: } 2 \mathrm{Ta}+10 \mathrm{OH}^{-} \rightarrow \mathrm{Ta}_{2} \mathrm{O}_{5}+5 \mathrm{H}_{2} \mathrm{O}
$$

The formation of Tantalum Oxide would results in loss of adhesion property and would release the electrodes, causing such defects to be formed. 


\subsection{Discussion}

From our experimental results with the developed device using human cheek cells as samples, we observed that both pulse width and applied electric field determines effects experienced by the cells. For micron width pulses, little or no effects were observed with even threshold electric field. As the electric pulse width becomes larger, electroporation phenomenon becomes evident. Electroporation phenomenon with relatively narrow pulse width did not cause any morphological changes in cell shape, size or orientation. However, relatively wider pulses with width in tens or a hundred of ms range, cell membrane deformation were noticed. As the electric field duration was extended over several hundreds of $\mathrm{ms}$, cell membrane structure were destroyed causing electrical lysis. However, released DNA were detected with pulse duration of $5 \mathrm{~s}$. A possible explanation is that this duration was required to break nuclear membrane that results in release of DNA. Smaller pulses lysing cell membrane might not lyse nucleus membrane, hence DNA was not released. Such observation is sketched in Figure 5.48.

Note that nucleus membrane composes of a double bilayer lipid membrane, hence the nucleus membrane should be stronger than cell membrane and harder to lyse. To be able to lyse nucleus membrane, it is reasonable to require a longer pulse width compared to that required for cell lysis.

\subsection{Summary}

We have designed and tested this microfluidic device that has several novel design attributes, like interdigitated electrodes underneath channel surface, and cell ports directly above microchannels. We have demonstrated EP with pulses as small as $5 \mathrm{~V}$ magnitude and $1 \mu$ s duration for a non-invasive cell. Rate of stained pixel reduction is found to be dependent on buffer solutions, indicating EP dependency on buffer fluids for effective utilization. EL experiments released DNA from cells, but electrode 
Ea Applied electric field

$E_{c}$ Critical electric field

ta Applied pulse width

tc Critical time for lysis
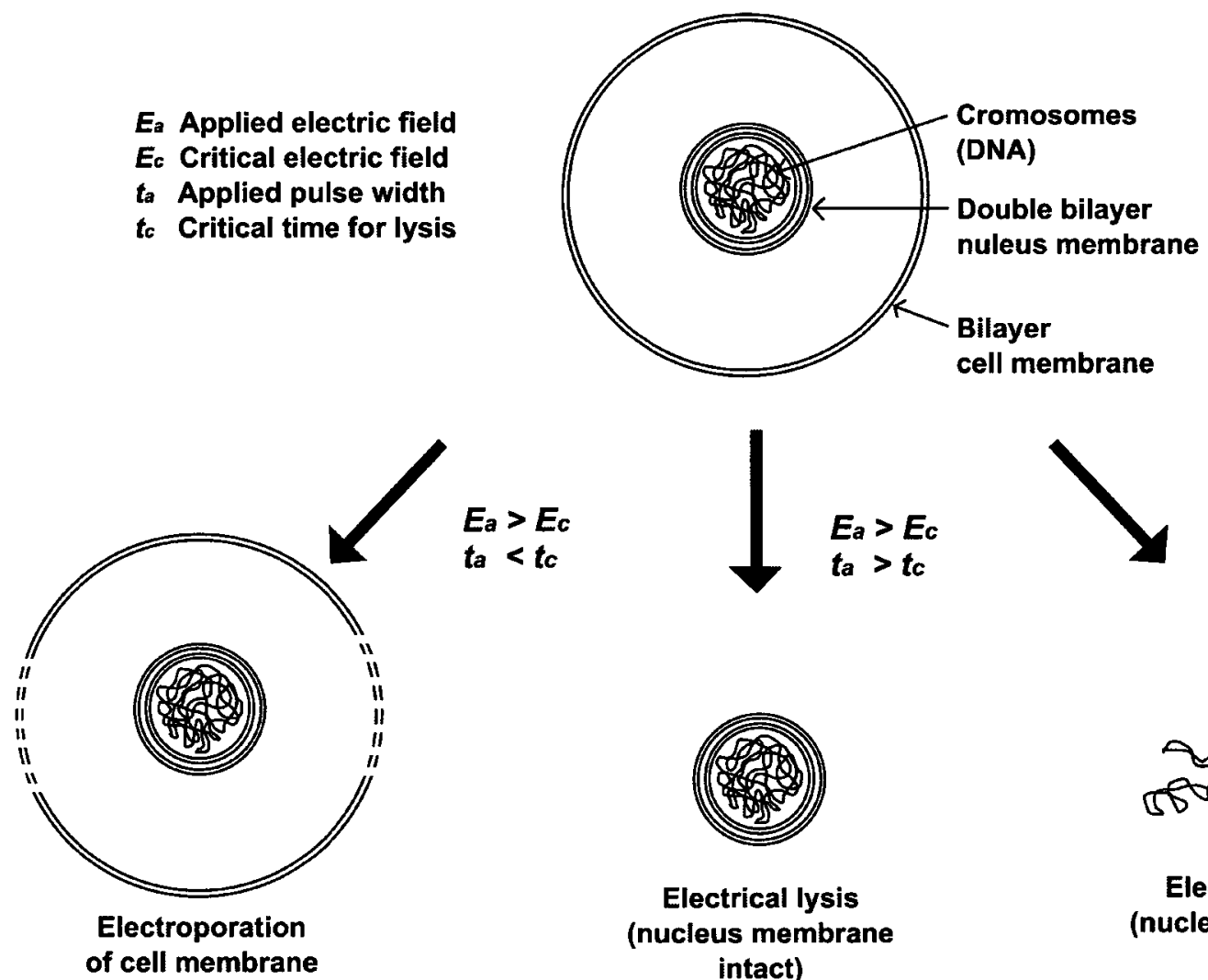

$E_{a}>E_{c}$

ta $<$ tc
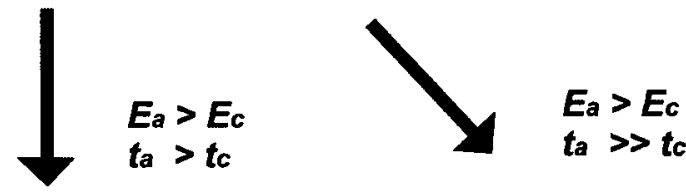

Figure 5.48 Sketches showing effect of applying above critical electric field pulses of different widths on a human cell causing electroporation, electrical lysis and nucleus membrane disintegration. 
breakdown limited extensive experimental study. Continued experiments are ongoing to overcome such limitation. 


\section{Chapter 6}

\section{Analysis}

In this chapter, we discuss our effort to improve the present state of knowledge and current approach of cell modelling. In particular, the first section discusses few illustrative diagrams that are developed during this research to explain electroporation dynamics conceived through literature review. The next section details an improvement of the current electric circuit model of cell to incorporate a pore model to represent membrane pores during electroporation. The last section proposes two quantification term of Electroporation (EP) that allows comparison of various cases of EP.

\subsection{Illustrative Diagrams to Represent Electropo- ration and Electrical Lysis Dynamics}

In this section, three illustrative diagrams are presented that are developed through this research. This illustrative diagrams integrates information or concept in a readily conceivable form. A detailed description of these diagrams are provided here.

Figure 6.1 is developed to illustrate a state diagram representation of the primary events during EP and Electrical Lysis (EL) of a cell exposed to a threshold electric 


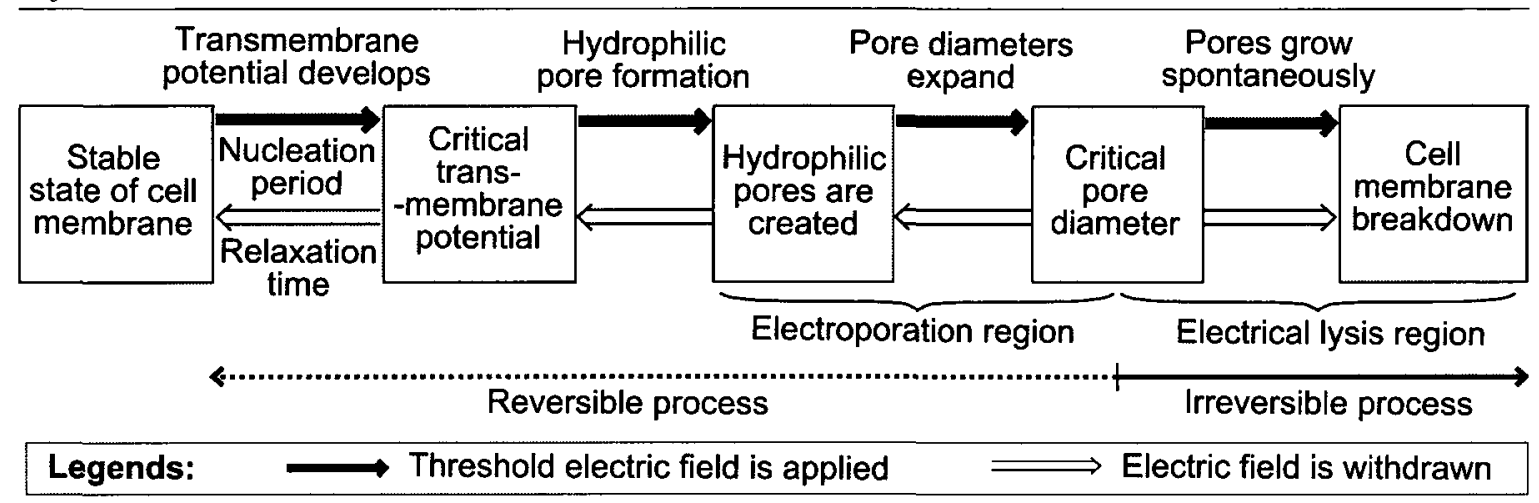

Figure 6.1 A state diagram representing the sequence of events involved in the dynamics of EL process. Here, the states are represented as rectangles and transitions are indicated using arrows. The solid arrows represent transitions due to application of an electric field above the threshold value. The hollow arrows represent spontaneous transitions after the applied electric field is withdrawn. The process is reversible until the critical pore diameter, after which pores grow spontaneously and an irreversible process is initiated. The zone between pore formation and pores attaining critical diameters is denoted by EP region, and the zone beyond the critical pore diameter is termed as the EL region

field. Figure 6.2, adapted by combining two figures from [1], is a graphical representation of membrane entropy $(\xi)$, due to reorientation of phospholipids, against elapsed time $(t)$ that illustrates some representative temporal paths (or traces) of entropies as various electric fields are applied or withdrawn. These two figures together are discussed below.

- As an electric field beyond the threshold value is applied on a cell, the stable state of phospholipids within the cell membrane is disrupted and a transmembrane potential develops across the membrane that increases the entropy of membrane structural organization.

- When this transmembrane potential reaches to a critical level, many small hydrophilic pores begin to form. This level of entropy is represented by $\xi_{1}$ in Figure 6.2. The corresponding time required are nucleation periods $\left(t_{n 1}, t_{n 2}\right.$, $\left.t_{n 3}\right)$. At this entropy, the reorientation force due to the applied electric field (eg. $\left.E_{t h 1}, E_{t h 2}, E_{t h 3}\right)$ is greater than the orientation force due to hydrophobicity of 


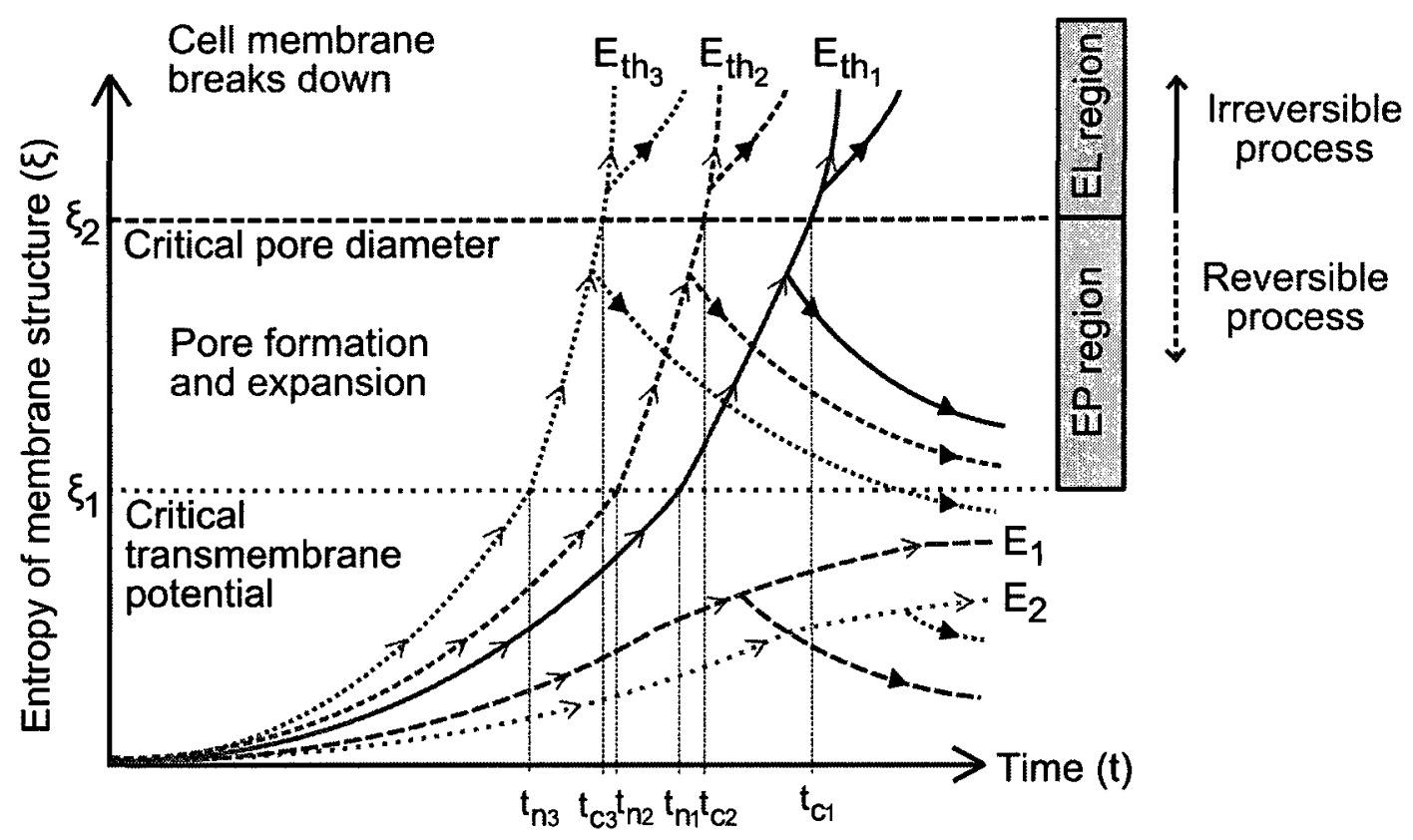

\section{Legends: \\ $\longrightarrow$ Path followed as an electric field is applied \\ $\rightarrow$ Path followed as the electric field is withdrawn}

$$
\begin{gathered}
E_{t h_{3}}>E_{t h_{2}}>E_{t h_{1}}>E_{t h} \\
\text { and } E_{2}<E_{1}<E_{\text {th }}
\end{gathered}
$$

Figure 6.2 Entropy of a cell membrane structure is drawn qualitatively as a function of elapsed time $(t)$. The paths denote traces of entropy for various applied electric field or traces as the electric field is withdrawn. The nucleation periods are denoted $\left(t_{n 1}, t_{n 2}, t_{n 3}\right)$. The critical times $\left(\mathrm{eg} . t_{c 1}, t_{c 2}, t_{c 3}\right)$ to achieve critical pore diameter reduce with increasing electric field strengths (i.e. $\left.E_{t h 1}, E_{t h 2}, E_{t h 3}\right)$ above the threshold electric field $\left(E_{t h}\right)$. (Adapted from [1].)

phospholipids. The formation of these new pores indicate EP region within the process. If the applied electric field (eg. $E 1, E 2)$ is lower than the threshold value, $\xi_{1}$ entropy level would never be reached.

- If the threshold electric field sustains, the newly formed pores grow in diameter as the entropy increases. When pore diameters reach the critical pore diameter for resealable range, the EP region ends and EL region begins. The corresponding entropy is represented by $\xi_{2}$ in Figure 6.2. The process is reversible up to this level, after which the process becomes irreversible. The critical time duration (eg. $t_{c 1}, t_{c 2}, t_{c 3}$ ), defined as the time required to reach $\xi_{2}$ level of entropy, 
decreases as the applied electric field (i.e. $E_{t h 1}, E_{t h 2}, E_{t h 3}$ ) is increased beyond the threshold electric field.

- If the applied electric field is withdrawn when the entropy is below $\xi_{2}$, the membrane entropy is decreased and pores reseal. If the electric field is withdrawn after the entropy exceeds $\xi_{2}$, the membrane entropy continues to increase and pores grow spontaneously, leading to destruction of the cell membrane structural organization resulting cell lysis.

The discussed dynamics, even though generally accepted, can not resolve some experimental observations. For example, it is reported that the membrane pole towards the positive electrode side (the hyperpolarized portion of the cell membrane) shows formation of pores more aggressively compared to the pole towards the negative electrode side (the depolarized membrane) [64]. However, the dynamics mentioned above assumes symmetrical deformation on both poles which falls short to explain such observations.

In an effort to explain this observation, reorientation of phospholipids within cell membranes due to the threshold electric field is illustrated using molecular dynamics approach for both hyperpolarized and depolarized membranes in Figure $6.3[2,3]$. Hyperpolarized section of the membrane is more negatively charged side, which occurs towards positive electrode. Similarly, depolarized section of the membrane is more positively charged side, which occurs towards negative electrode. We demonstrate here that when we consider reorientation of phospholipids on both hyperpolarized and depolarized sections of a cell membrane, this phenomenon can be explained as discussed below.

The phospholipids, acting as electric dipoles, arranged in stable cell membrane formation (Figure 6.3a) experience reorientation force due to the applied threshold electric field that induces charged ions along the cell membrane (Figure 6.3b) $[2,86]$ ). This stress to rotate around each individual phospholipid results in pore formation (Figure 6.3c). Here each phospholipid molecule is assumed as a rigid unit. It is in- 


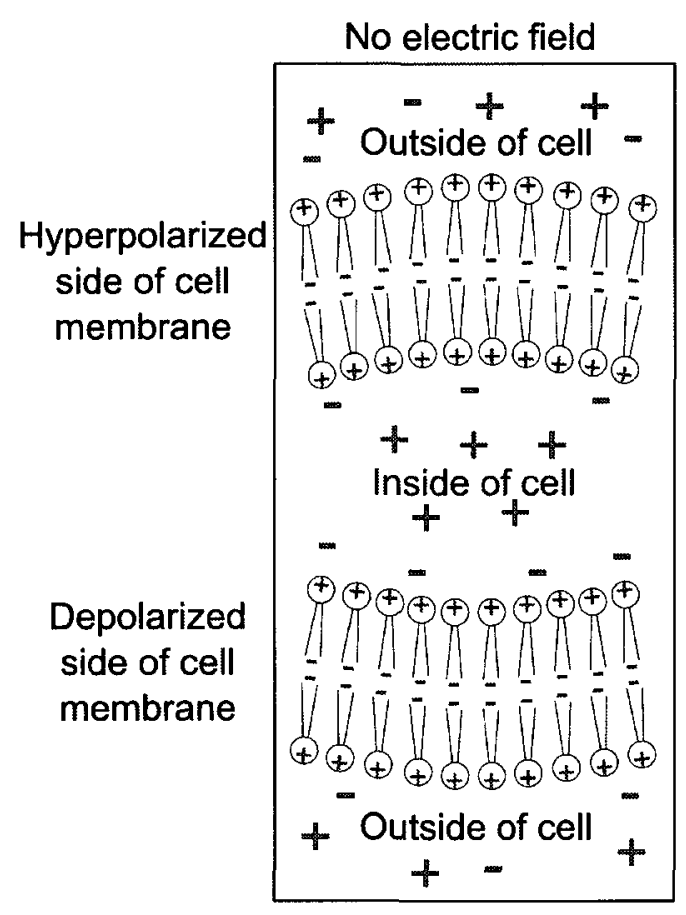

(a) Stable state of bilayer membrane
Electric field applied

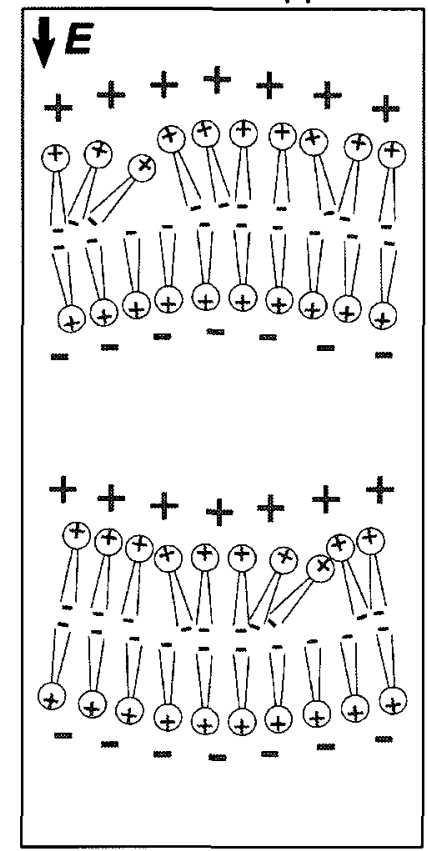

(b) Reorientation of phospholipids

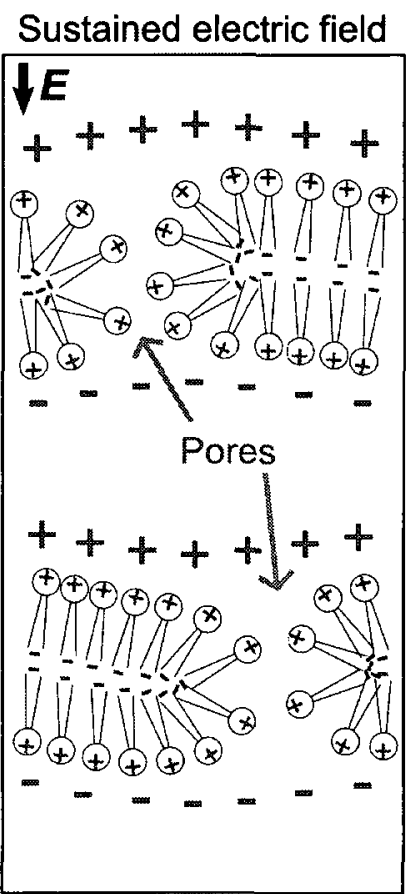

(c) Formation of hydrophilic pores

Figure 6.3 Reorientation of phospholipids of a bilayer membrane in hyperpolarized and depolarized side of a cell under a threshold electric field $(\boldsymbol{E})$ causing pores to form. The arrows in (b) and (c) indicate the directions of the applied electric field. (Adapted from $[2,3]$.)

teresting to note that only the outer layer of the hyperpolarized membrane and the inner layer of the depolarized membrane experience the reorientation force (Figure $6.3 \mathrm{~b})$. As the inner layer of a cell membrane, compared to the outer layer, is expected to be densely packed with phospholipids and might be bound to cytoskeleton, more reorientation of phospholipids should occur at the hyperpolarized side of the membrane compared to that at the depolarized side. Such fact would lead to a higher pore formation tendency towards hyperpolarized membrane compared to depolarized side that can justify the reported observation [64]. 


\subsection{Improvement to the Current Electric Circuit Model of Cell Membrane}

In this section, we discuss our effort to improve the current electric circuit model of cell membrane. Towards this goal, we have identified the need to introduce membrane voltage induced pore mechanism, as well as changing membrane potential with time after electroporation. The obtained results reflect properly the experimental results reported earlier, which are unexplainable with the current model.

\subsubsection{Current electric circuit model of cell membrane}

A cell exposed to an electric field during EP can be modelled by the use of electric circuit components, such as resistors and capacitors. Initial scheme of electric circuit modeling of a cell was formulated by Schwan [120], later developed by Schoenbach et al $[4,104,105,109,114,115,136]$. The electric circuit model of a cell is shown in Figure 6.4. Here, the cell membranes are represented by capacitors $\left(C_{m}\right)$ in parallel with resistors $\left(R_{m}\right)$, and the intracellular fluid is modelled as resistive component $\left(R_{c}\right)$ [105]. A voltage source in series with the membrane resistors are sometimes modelled to represent voltage-gated channels within the membrane, which controls flow of ions through the membrane depending on the transmembrane potential $[109,114,115]$. Electric circuit model has the advantage of determining cell behavior under various types of excitations [4]. However the model suffers from several drawbacks, such as:

1) The model parameters are not extractable from spherical cell structure.

2) Fixed resistance modelling the transmembrane resistance ignores membrane pore resistances and relevant dynamics. 


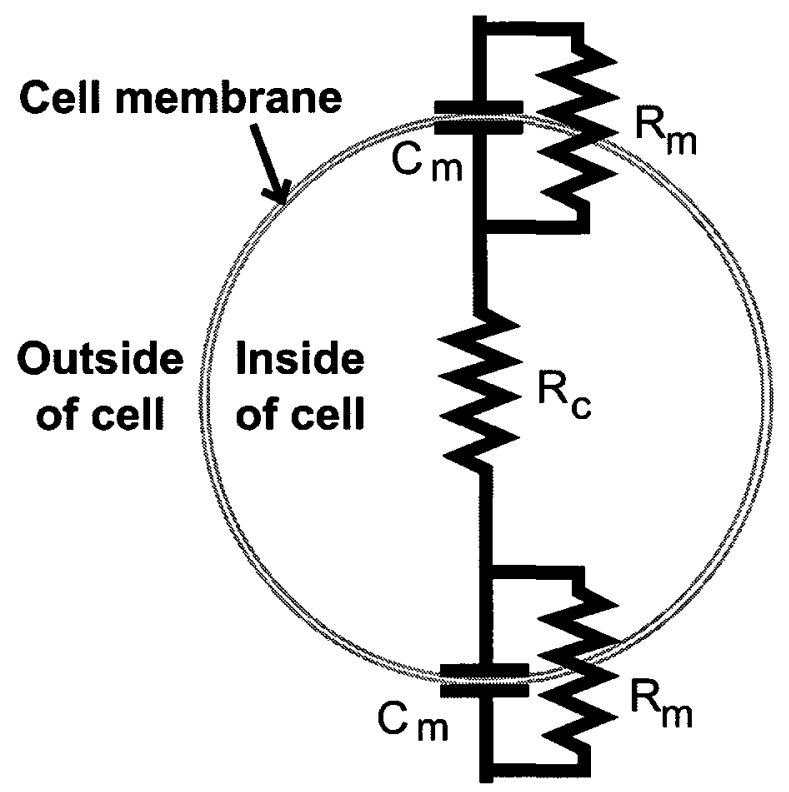

Figure 6.4 Current electric circuit model of a cell, where the cell membrane is represented by a parallel combination of a capacitor $\left(C_{m}\right)$ and a resistor $\left(R_{m}\right)$, while the cytoplasm inside the cell is modelled with a resistor $\left(R_{c}\right)$.

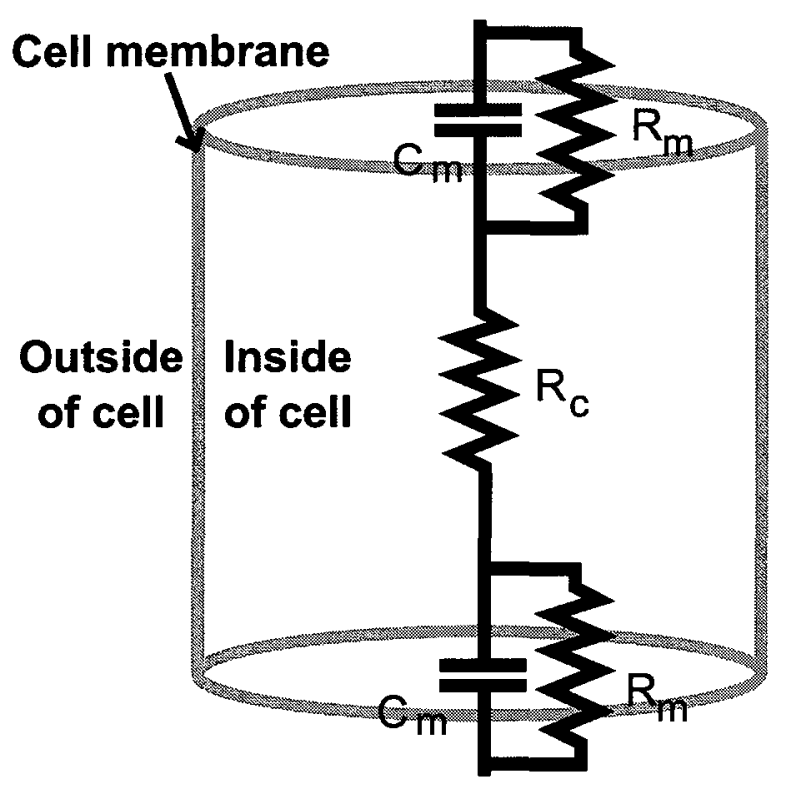

Figure 6.5 Cylindrical model of a cell converted from the spherical cell that allows determination of model parameters of the electrical circuit model. 


\subsubsection{Model parameter extraction with a cylindrical model}

The spherical cell structure poses several challenges in determining the cell model parameters:

1) Calculating membrane parameters $\left(C_{m}, R_{m}\right)$ is not directly achievable as the surface area to be considered is not defined in a spherical cell model.

2) The intracellular resistance $\left(R_{c}\right)$ is infinite if point contact is assumed for a sphere, hence again an undefined contact area is to be considered.

To resolve these issues, a cylindrical model is developed where the radius of the base is $r_{c}$ and the height of the cylinder is $h_{c}$. With this model, cell area exposed to the high electric field is defined by $\pi r_{c}^{2}$. In fact, most of the cells in practice resemblance cylindrical shaper, rather than spherical shapes. For the same volume of the spherical cell with radius $r_{a}$ and the cylindrical model, we find the following relationship:

$$
r_{c}^{2}=\frac{4 r_{a}^{3}}{3 h_{c}} .
$$

Hence for a certain $h_{c}, r_{c}$ can be determined, whereas $h_{c}$ is dependent on the cell area that experiences the high electric field. As ratio of cell area experiencing the high electric field increases, $r_{c}$ increases and $h_{c}$ decreases. Note that with this modification, we are able to isolate cell membrane affected or not affected by the applied electric field while maintaining the same volume. In fact, cylindrical model is more appropriate to represent practical cells, because the shape of most cells in nature are more comparable with a cylindrical structure. The cell area of the base of the cylindrical model contribute to the capacitance $\left(C_{m}\right)$ and resistance $\left(R_{m}\right)$ of the equivalent circuit model, where as the sidewall does not effect any parameter of the equivalent circuit model (Figure 6.5). In addition, the cylindrical model allows calculation of $R_{c}$.

Using this model, we can determine various electric circuit model parameters from 
physical parameters as follows:

$$
\begin{gathered}
C_{m}=\frac{\varepsilon_{m} \varepsilon_{0} \mathbf{V}_{c}}{d_{m} h_{c}} \\
R_{m}=\frac{\rho_{m} d_{m} h_{c}}{\mathbf{V}_{c}} \\
R_{c}=\frac{\rho_{c} h_{c}^{2}}{\mathbf{V}_{c}}
\end{gathered}
$$

where $\varepsilon_{m}$ is the relative permittivity of the cell membrane, $\varepsilon_{0}$ is the absolute permittivity of the free space $\left(8.85 \times 10^{-12} \mathrm{~F} / \mathrm{m}\right), d_{m}$ is the thickness of the cell membrane, $\rho_{m}$ and $\rho_{c}$ are membrane and cytoplasm conductivities, respectively, and $\mathbf{V}_{c}=\frac{4}{3} \pi r_{a}^{3}$ is the volume of the cell. As an initial approximation, $h_{c}$ is assumed to be $2 r_{a}$, which implies $r_{c}=0.8 r_{a}$. In addition to cell model parameters, buffer parameters are calculated using the following expressions (Figure 6.6):

$$
\begin{gathered}
R_{S B}=\frac{\rho_{B}\left(l_{c h}-h_{c}\right)}{A_{c h}} \\
C_{B}=\frac{\varepsilon_{B} \varepsilon_{0} A_{c h}}{l_{c h}} \\
R_{P B}=\frac{\rho_{B} h_{c}^{2}}{A_{c h} h_{c}-V_{c}}
\end{gathered}
$$

where $\rho_{B}$ is the buffer resistivity, $\varepsilon_{B}$ is the relative permittivity of the buffer medium, $l_{c h}$ is the gap between electrodes in the microchannel, $A_{c h}$ is the cross section area of the microchannel, $R_{S B}$ is the buffer resistance between the electrodes and cell membranes, $R_{P B}$ is the buffer resistance in parallel to the cell, and $C_{S B}$ is the buffer capacitance. To calculate the model parameters, we have used the values given in Table 6.1 [4]. The value of $\rho_{m}$ is very high (approaching infinite), quantified as $10^{12} \Omega-m$. All other parameters are design specific. 


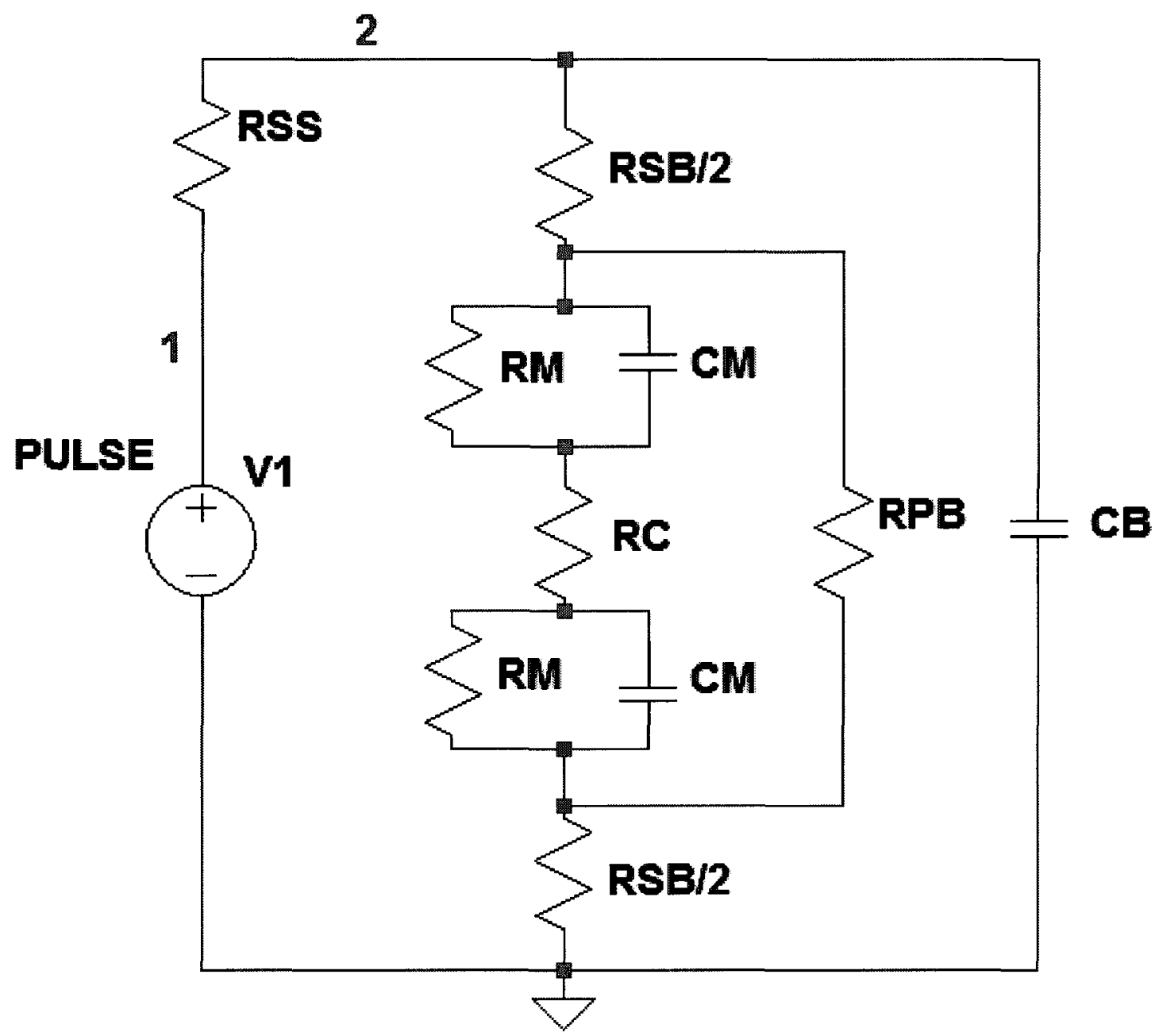

Figure 6.6 The complete electrical circuit including a model of a cell inside a micro-channel 
Table 6.1 Model parameters used to calculate component parameters of the electric circuit model from literature [4]

\begin{tabular}{lll} 
Parameter & Value & Unit \\
\hline$r_{a}$ & 5 & $\mu m$ \\
\hline$\rho_{c}$ & 1.67 & $\Omega-m$ \\
\hline$\rho_{B}$ & 1.67 & $\Omega-m$ \\
\hline$\varepsilon_{B}$ & 80 & - \\
\hline$\varepsilon_{m}$ & 8 & - \\
\hline$d_{m}$ & 5 & $\mathrm{~nm}$
\end{tabular}

\subsubsection{Modelling of pore resistance}

One of the major limitations of the current electric circuit model of a cell in an electric field (Figure 6.4) is that there is no pore resistance component in the membrane model ( $R_{m}$ represents membrane resistance). We propose a membrane pore resistance model in this section that will replace the current membrane resistance $\left(R_{m}\right)$ in the electric circuit cell model. The resistance of this pore model will change dynamically from a very high value (membrane resistance) to a low value (pore resistance). Furthermore, the pore resistance will be dependant on the transmembrane potential, as well as decrease as the pores would grow with respect to the elapsed time of excitation, as prescribed by the relevant dynamics $[1,102,104,106,109]$.

Pores developed during EP are hydrophilic nature and do not exist in stable state of membrane condition (Figure 6.3a). Hence transmembrane resistance should be very high at steady state. As the transmembrane potential reaches $1 \mathrm{~V}$, membrane pores start to form. These pores (Figure 6.3c) can be modelled using a cylindrical approximation with pore diameters as $r_{p}$ and the pore height equal to cell membrane thickness, $d_{m}$ (Figure 6.7). These hydrophillic pores contain fluids either from buffer 


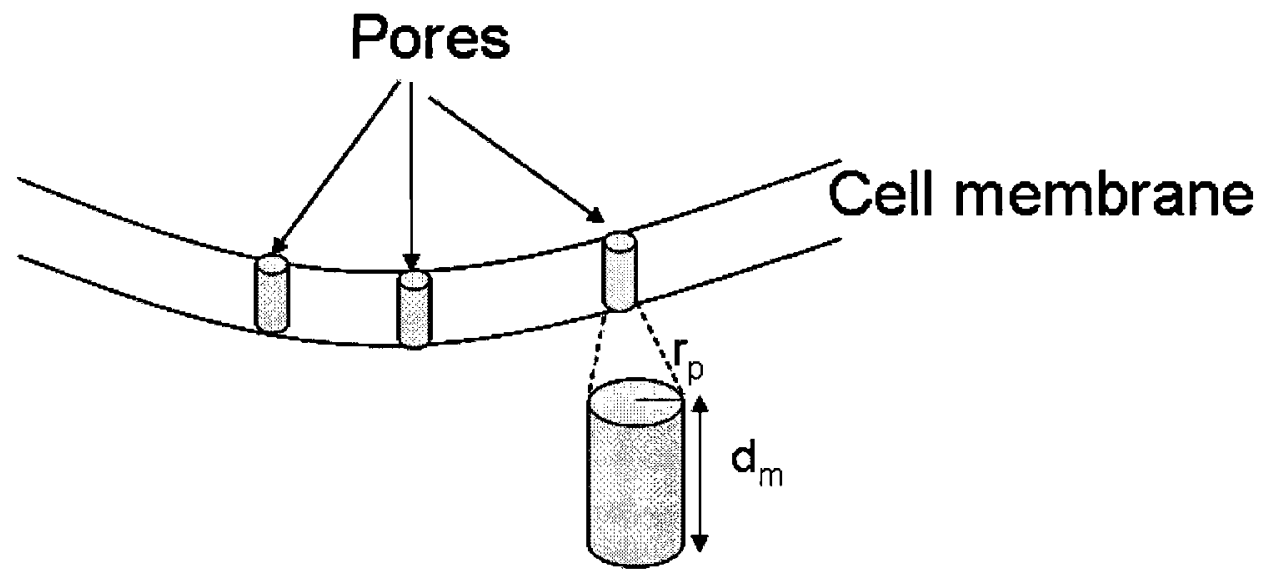

Figure 6.7 A cylindrical model of cellular membrane pores formed during EP.

or from cytoplasm, both has the same resistivity (Table 6.1). The resistance of these cylindrical pores can be calculated by,

$$
R_{p}=\frac{\rho_{B} d_{m}}{\pi r_{p}^{2}}
$$

The pore radius is a function of pulse duration. However there is no previous study conducted on this relationship. As a first approximation, we assume a linear relationship in this proposed model. Implementing other relationship is trivial. The number of membrane pores $\left(\mathbf{n}_{p}\right)$ is considered to be a function of the applied potential. The total transmembrane resistance $\left(R_{T M}\right)$ for $\mathbf{n}_{p}$ pores can be calculated as:

$$
R_{T M}=\frac{R_{p}}{n_{p}}=\frac{\rho_{B} d_{m}}{\mathbf{n}_{p} \pi r_{p}^{2}}=\frac{K_{1}}{\mathbf{n}_{p} r_{p}^{2}}
$$

Here $K_{1}=\rho_{B} d_{m} / \pi$ is a proportionality constant. The value of $K 1$ using the parameters given in the Table 6.1 is $2.7 \times 10^{-9}$. The calculated values of the total transmembrane resistance $\left(R_{T M}\right)$ for $r_{p}$ from $1 \mathrm{~nm}$ to $20 \mathrm{~nm}$ and $n_{p}$ of 1000,2000 , and 5000 using this expression are plotted in Figure 6.8.

To find an electrical equivalent model of the membrane pore resistance, note that the transmembrane resistance is inversely proportional to the square of pore radius. In addition, pore radius will only increase when the applied potential is sustained. These characteristics are transferred in terms of electric circuit components in the 


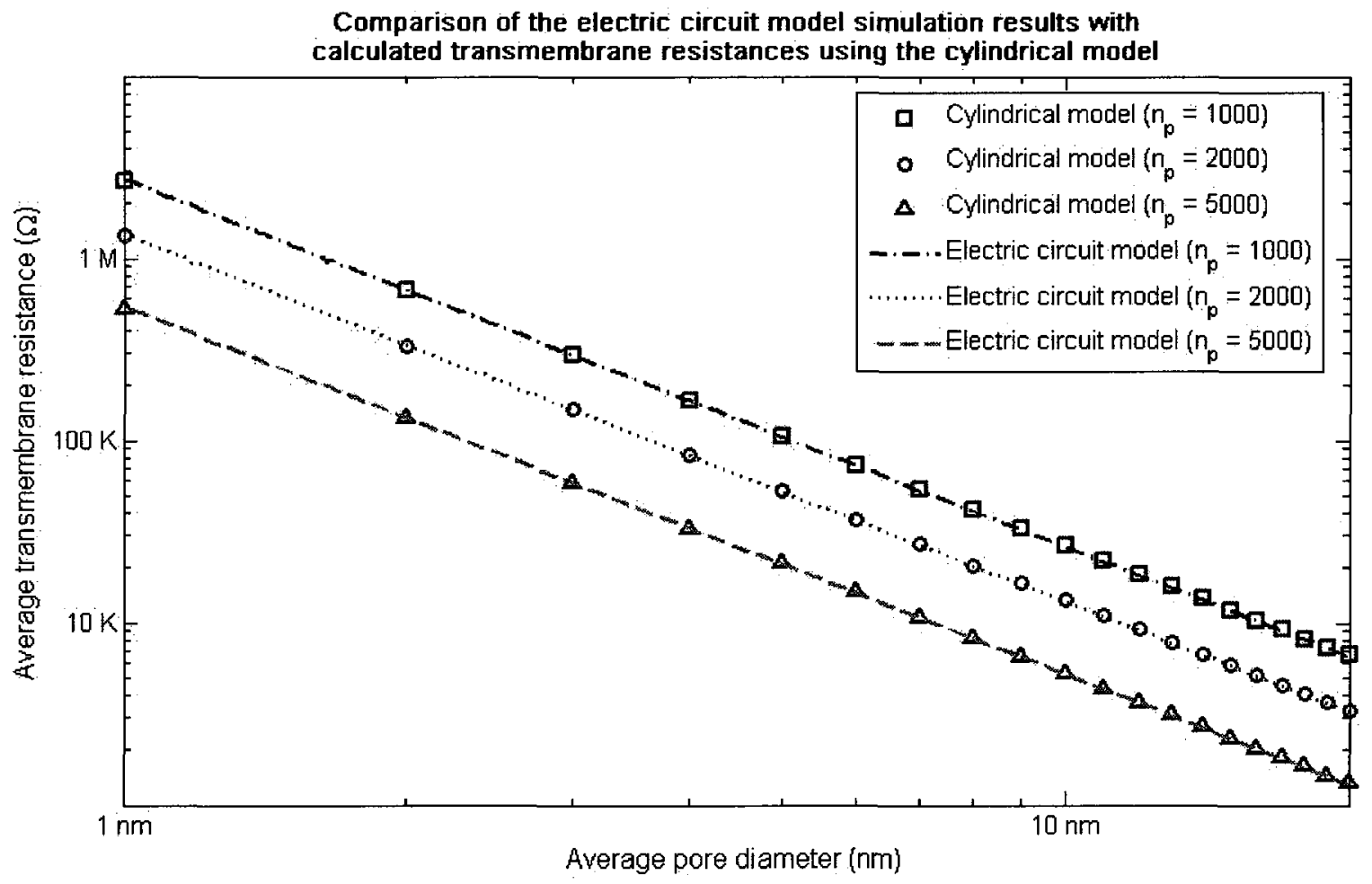

Figure 6.8 Comparison of total average membrane resistance $\left(R_{T M}\right)$ calculated using the cylindrical pore model and the electrical circuit model for typical pore diameters and the number of pores $\left(\mathbf{n}_{p}\right)$. 


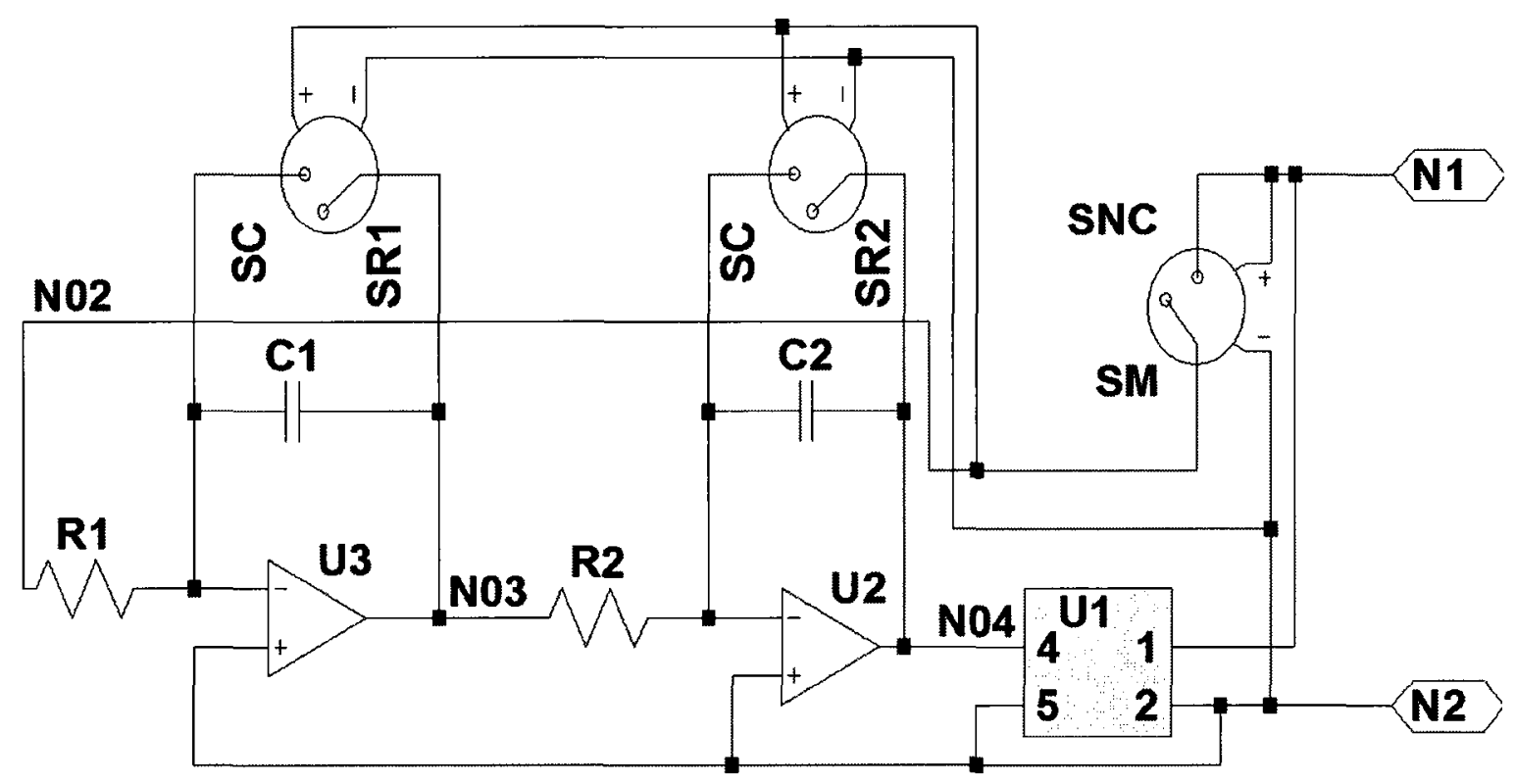

.subckt opamp 123

G1 $2321\{10 \mathrm{~K}\}$

R3 321.

C3 $32\{10 \mathrm{~K} / 1 \mathrm{~T}\}$

.ends opamp

.subckt vcr_pore 1245

eres 13 value $=\left\{2.7 p^{*} \mid(\right.$ vsense $\left.) /(V(4,5))\right\}$

vsense $32 \mathrm{dc}$ OV

.ends vcr_pore

.model snc vswitch(RON=0.1, roff $=1 \mathrm{e} 20$, von $=1$, voff $=0.99$ )

.model sc vswitch(RON=0.1, roff $=1 \mathrm{e} 20$, von $=0.05$, voff $=0.1)$

Figure 6.9 The electrical model of transmembrane resistance due to reversible pores formed during EP. The membrane resistance between $\mathrm{N} 1$ and $\mathrm{N} 2$ reduces drastically as the potential reaches the threshold transmembrane potential. 
model shown in Figure 6.9 by a two-stage cascaded integrators (U3 and U2) that drives a voltage controlled resistor component (U1). The input of the first integrator is controlled by a voltage controlled switch (SM) that turns on as the transmembrane voltage (voltage between $\mathrm{N} 1$ and $\mathrm{N} 2$ ) exceeds $1 \mathrm{~V}$, the critical voltage required to initiate pores. Hence below this critical transmembrane potential, the resistance between $\mathrm{N} 1$ and $\mathrm{N} 2$ is very high ( $\mathrm{T} \Omega$ range). Above the critical transmembrane potential, the resistance decreases (as the pore radius would increase) at the inverse rate of the square of the elapsed time.

The proportionality constant $(K)$ of the voltage-controlled resistance (U1) is shown as $2.7 \mathrm{p}$ in Figure 6.9. This value is calculated for $\mathbf{n}_{p}=1000$ using the following relationship:

$$
K=\frac{K_{1}}{\mathbf{n}_{p}} .
$$

The resistance values obtained using this relationship for different number of pores correspondingly matched with the values calculated using the expression (6.9) and plotted alongside the previously plotted data (Figure 6.8). The value of the terminal resistance of the voltage controlled resister (Node 1 and 2 of U1) is $K / V_{N 04}$. Thus $V_{N 04}$ needs to be equivalent to $r_{p}^{2}$ as dictated by expression (6.9). For a constant transmembrane potential $\left(V_{T M}\right)$, we can determine the voltage at N04 to be:

$$
V_{N 04}=\frac{V_{T M}}{R 1 C 1 R 2 C 2} \int_{0}^{t} \int_{0}^{t} d t d t=\frac{V_{T M} t^{2}}{2 R 1 C 1 R 2 C 2} .
$$

This expression shows a linear temporal relationship of pore radius that was assumed. The proportionality constant is a factor of square root of the transmembrane potential, as well as dictates the values of the resistances and the capacitances of the integrators. Thus the transmembrane potential can be represented as:

$$
R_{T M}=\frac{2 K R 1 C 1 R 2 C 2}{V_{T M} t^{2}} .
$$

Thus for a certain transmembrane potential above critical transmembrane potential, the transmembrane resistance is inversely proportional to the square of the elapsed time. 
To initialize (and reset) the integrators when transmembrane potential is below a certain small value (eg. $0.1 \mathrm{~V}$ ), two voltage controlled switches (SR1 and SR2) are introduced across the capacitors of the integrators.

\subsubsection{Simulation results}

Complete circuit diagrams of a cell model inside a micro-channel of the current model and the proposed model including power supply and necessary components to represent microchannel parameters are shown in Figure 6.10. Here V1 is the supply voltage, RSS is total resistance of internal resistance of the power supply and the electrode resistances, RSB represents the series resistance of the buffer, CB represents the capacitance across the microchannel electrodes, RPB represents the parallel resistance around the cell, and $\mathrm{RC}$ is the internal resistance of cytoplasm. The cell membrane is represented by a parallel combination of a capacitor (CM) with a resistor (RM) for the current model (Figure 6.10a), or with a pore model (X1) for the proposed model (Figure 6.10b). The two cell membranes in series can be modelled with a parallel combination of a resistor and a capacitor of following values:

$$
\begin{gathered}
R_{m}(c o m b)=2 R_{m}=2 R M, \\
C_{m}(c o m b)=C_{m} / 2=C M / 2 .
\end{gathered}
$$

In this case, the transmembrane potential across each membrane will be half of the potential recorded across the combined impedance. For the case of the developed pore model, all internal switching voltage levels are doubled to accommodate the effect.

Simulations were performed with a SPICE simulator (LTspice IV, Linear Technology Corporation) with the following parameters: $\mathrm{RSS}=100 \Omega, \mathrm{RSB}=1.67 k \Omega$, $\mathrm{RPB}=8.56 k \Omega, \mathrm{RC}=319 k \Omega, 2 \mathrm{RM}=190 * 10^{12} \Omega, \mathrm{CB}=0.1416 \mathrm{pF}, \mathrm{CM} / 2=0.37$ $\mathrm{pF}, \mathrm{R} 1=\mathrm{R} 2=100 \mathrm{M} \Omega$ and $\mathrm{C} 1=\mathrm{C} 2=1 \mu \mathrm{F}$. These were determined based on the modelling approach as discussed above and using values provided in Table 6.1. 

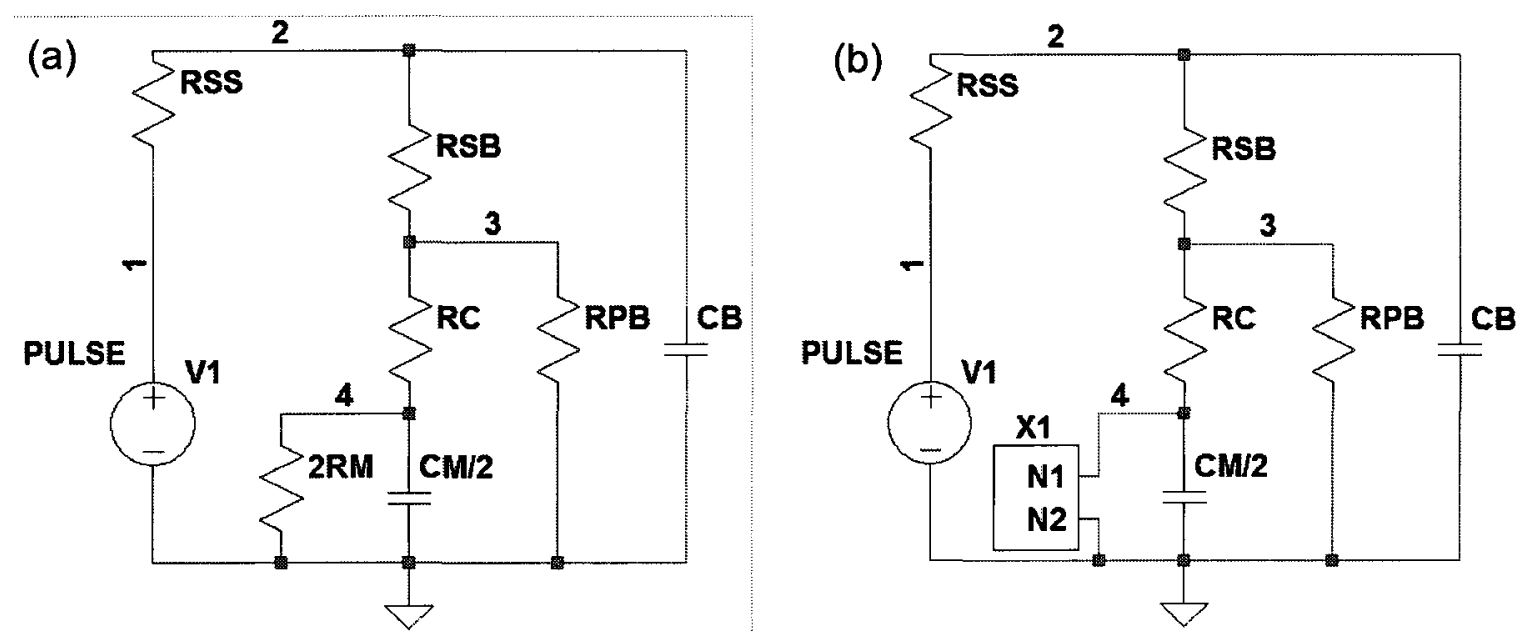

Figure 6.10 The complete circuit diagram for simulation of a cell inside a microchannel using (a) the current pore model with a fixed resistance model $(2 \mathrm{RM})$, and (b) the proposed pore model (X1). Here the cell is modelled using $\mathrm{RC}, \mathrm{CM} / 2$, the microchannel is modelled using RSB, RPB, and CB, and the power supply is represented by V1, and RSS.

Simulation results with the current model (Figure 6.10a) and the developed model (Figure 6.10b) are presented in Figure 6.11 to Figure 6.14.

Using the current electric circuit equivalent model of cell, the transient response is shown in Figure 6.11. As a pulse is applied, the transmembrane potential increases and reaches to a steady state voltage that is directly dependent on the applied voltage and the values of resistances in series, as the capacitors become fully charged. For example, in this simulation shown, the resulted transmembrane potential is $4.114 \mathrm{~V}$ with applied voltage of $10 \mathrm{~V}$. Note that the transmembrane resistance of cell membrane is constant throughout the simulation. However, with the formation of pores, transmembrane resistance is expected to decrease as conducting paths are generated, as the transmembrane potential is larger than $1 \mathrm{~V}$. Thus, EP phenomenon is not reflected from simulation of the current model, as the current model does not contain any element for pore model.

With our developed model, we have included dynamic pore model within the cell model. The simulation result with the same external conditions for current model is shown in Figure 6.12 for a comparison. A simulation result with one pulse is shown 


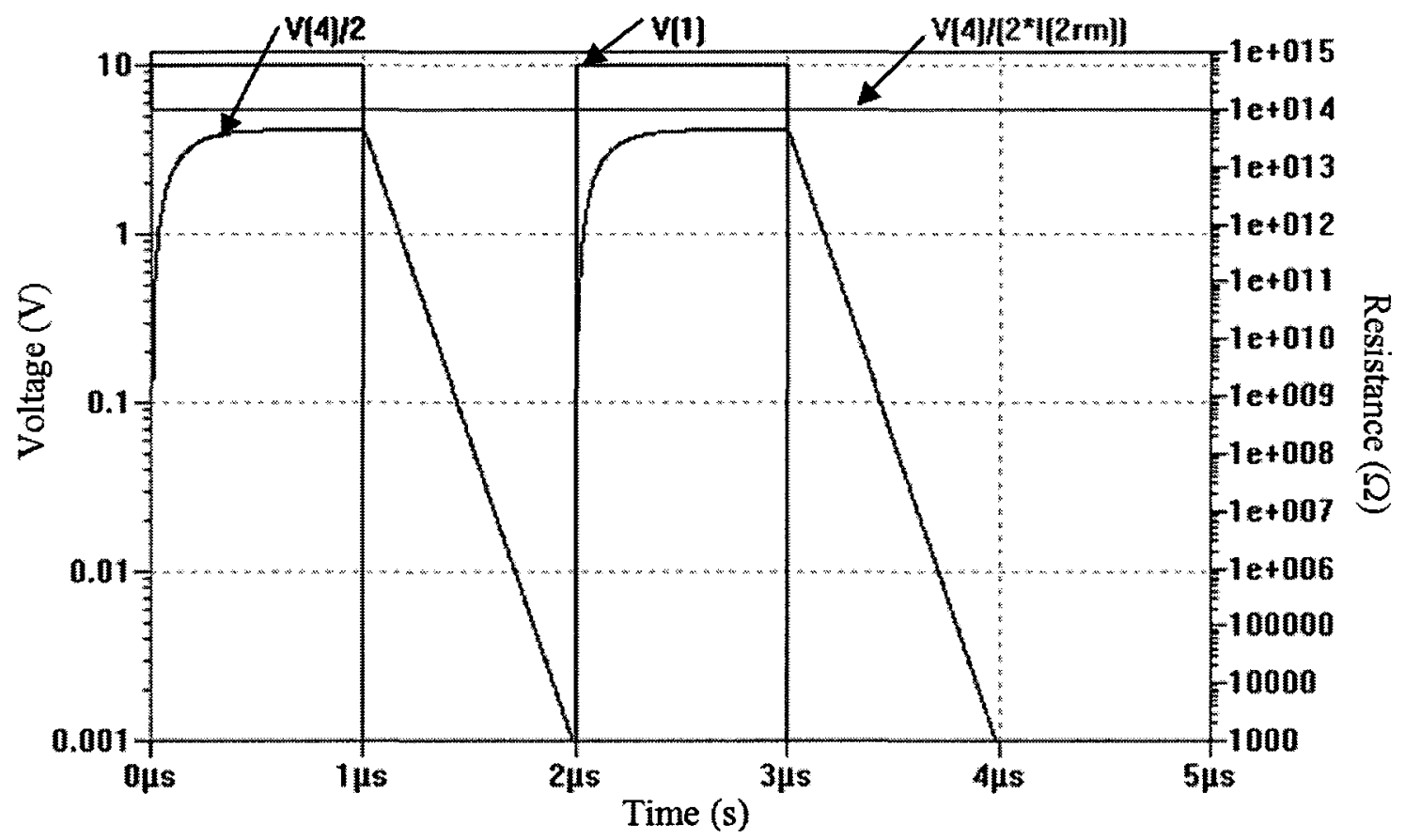

Figure 6.11 Simulation result of a cell exposed to 2 pulses of $1 \mu$ s duration using the current pore model. Here, $\mathrm{V}(1)$ represents the applied voltage, $\mathrm{V}(4) / 2$ represent transmembrane potential, and $\mathrm{V}(4) /\left(2^{*} \mathrm{I}(2 \mathrm{rm})\right.$ represents the membrane resistance. 


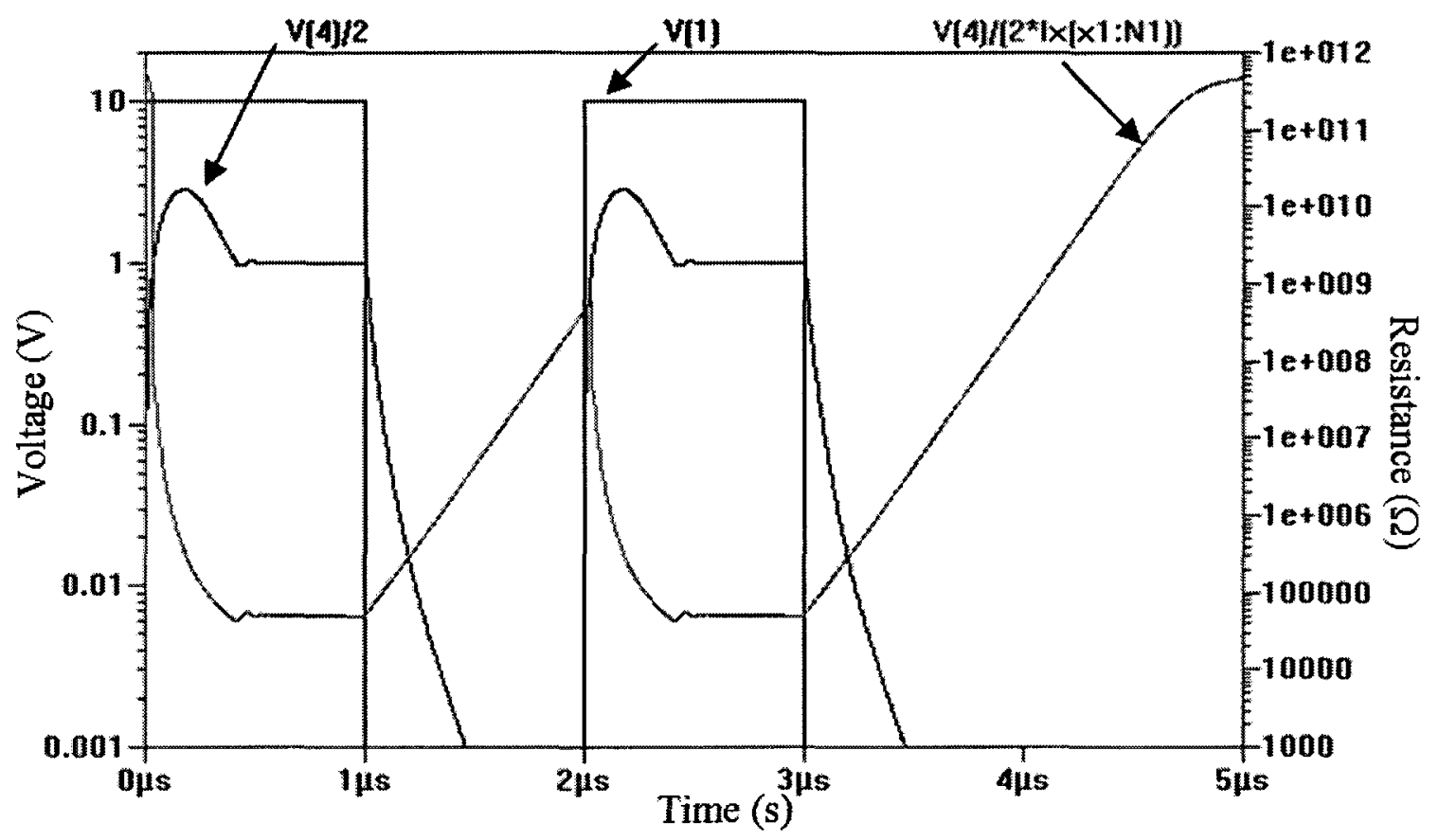

Figure 6.12 Simulation result of a cell exposed to 2 pulses of $1 \mu$ s duration using the developed pore model. Here, $\mathrm{V}(1)$ represents the applied voltage, $\mathrm{V}(4) / 2$ represent the transmembrane potential, and $\mathrm{V}(4) /\left(2^{*} \mathrm{I}(\mathrm{X} 1: \mathrm{N} 1)\right.$ represents the membrane (pore) resistance. 


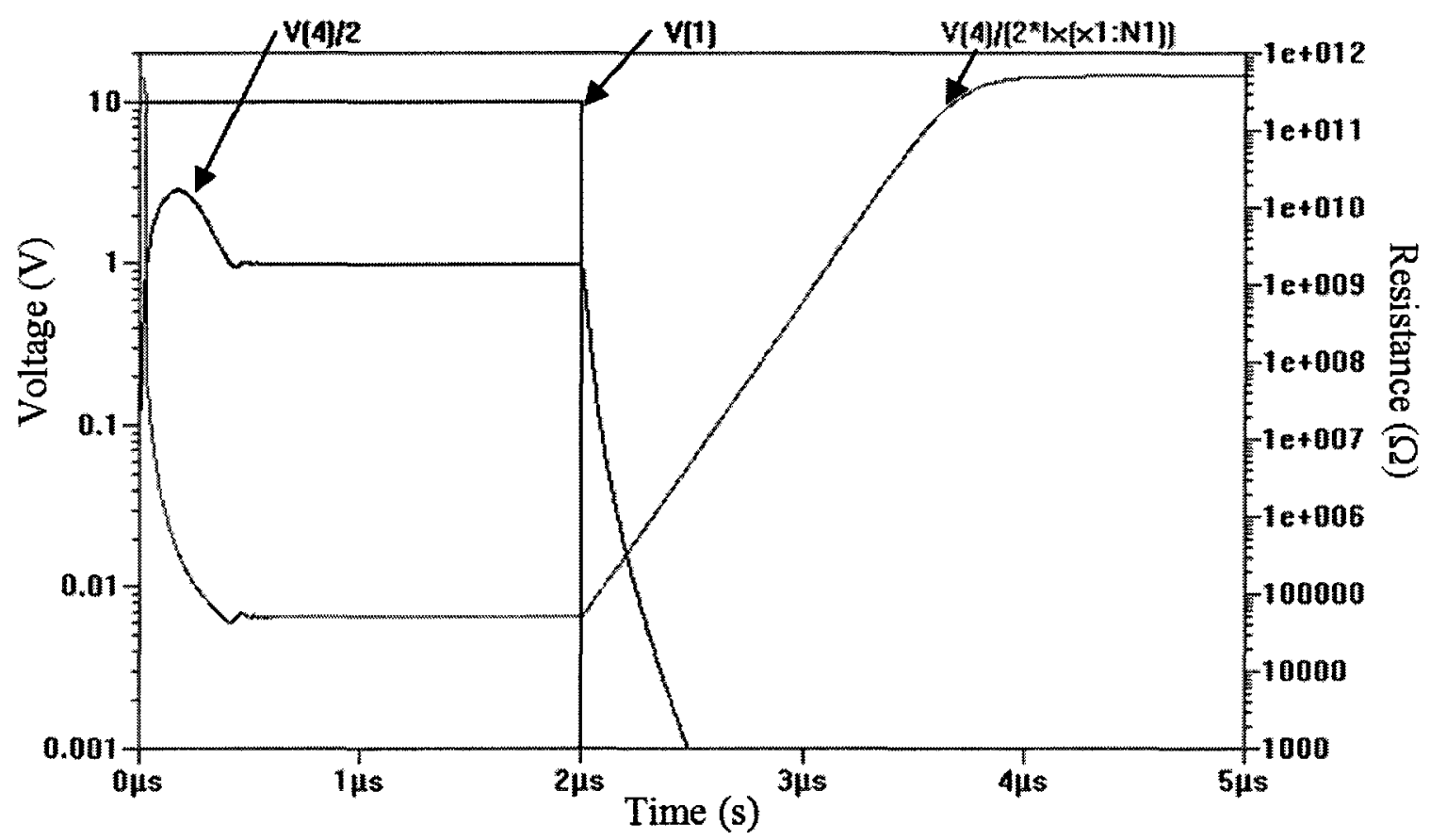

Figure 6.13 Simulation results of a cell exposed to a single pulse of $2 \mu$ s duration using the developed pore model. Here, $\mathrm{V}(1)$ represents the applied voltage, $\mathrm{V}(4) / 2$ represent the transmembrane potential, and $\mathrm{V}(4) /\left(2^{*} \mathrm{I}(\mathrm{X} 1: \mathrm{N} 1)\right.$ represents the membrane (pore) resistance. 


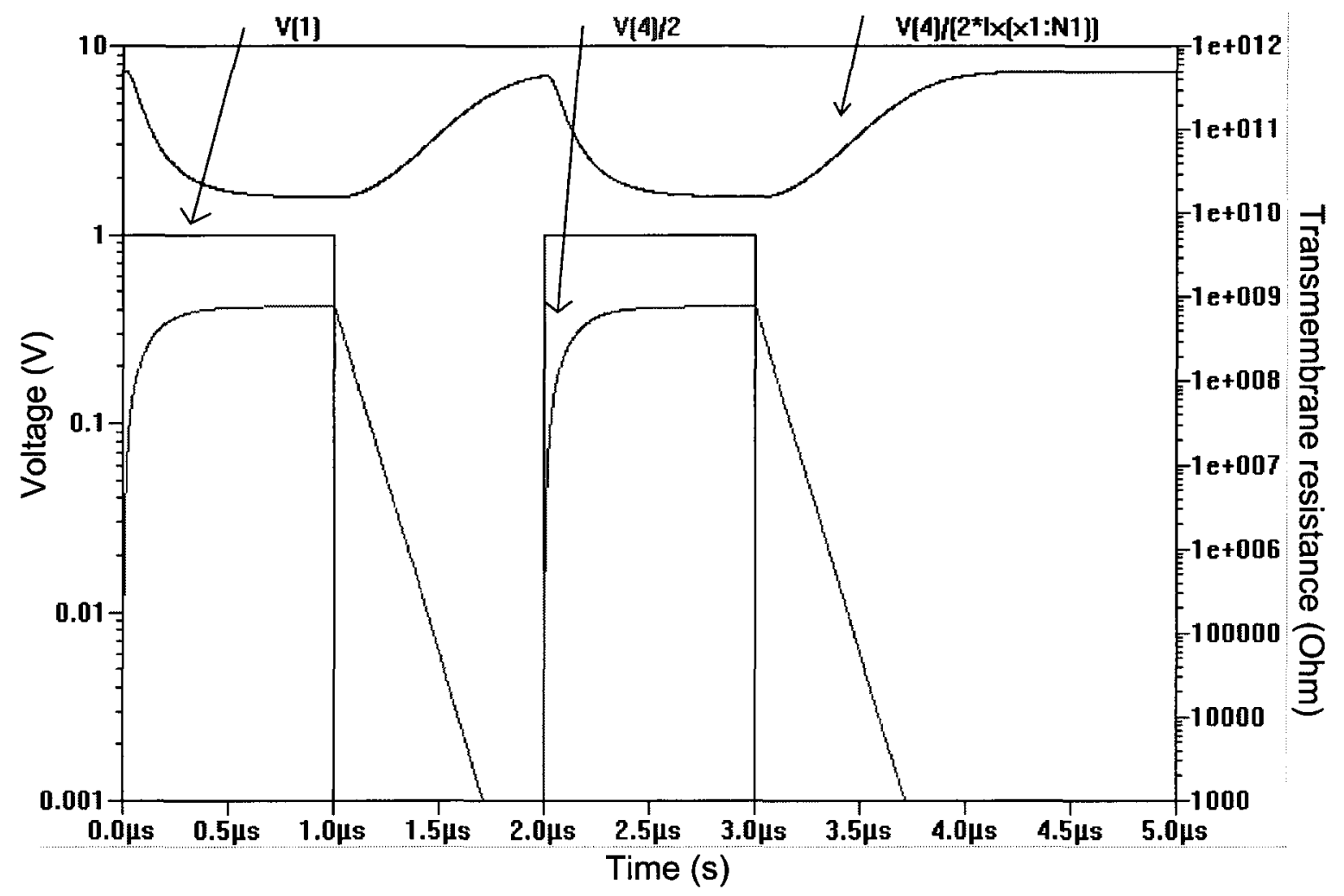

Figure 6.14 Simulation result of a cell exposed to 2 pulses of $1 \mu$ s duration using the developed pore model where no EP occurred, as the applied voltage is low. Here, $\mathrm{V}(1)$ represents the applied voltage, $\mathrm{V}(4) / 2$ represent the transmembrane potential, and $\mathrm{V}(4) /(2 * \mathrm{I}(\mathrm{X} 1: \mathrm{N} 1)$ represents the membrane (pore) resistance. 
in Figure 6.13. Using the proposed model, the transmembrane potential increases as the applied pulse is initiated. However, as transmembrane potential exceeds $1 \mathrm{~V}$, the transmembrane resistance reduces drastically, representing pore formation. This reduction of transmembrane resistance results in reduction of the transmembrane potential, as voltage drop across other resistive elements increase. If the transmembrane potential reduces to $1 \mathrm{~V}$ or lower, the membrane resistance starts to increase due to resealing of membrane pores. Thus the transmembrane potential eventually settles to $1 \mathrm{~V}$, irrespective of applied voltage - a constraint that can be predicted from the dynamics of EP. The initial rise of voltage (spike) observed represents transient pore formation (as the pores are formed and then expanded). Pore formation is also characterized by the reduction of transmembrane resistance. The settling of transmembrane potential represent a certain total pore area that results a transmembrane resistance that sustains a transmembrane potential of $1 \mathrm{~V}$. Note that the simulation results are plotted in logarithmic scales for both $y$-axes in these plots.

With the proposed model, if a low voltage is applied the resultant transmembrane potential does not reach the critical value of $1 \mathrm{~V}$ as shown in Figure 6.14. The transmembrane resistance also does not reduce as much as the cases of EP. This is expected from the dynamics as pores do not form in this case as critical transmembrane potential is never reached. The small decrease of transmembrane resistance is also consistent with the dynamics as the cell membrane is expected to be compressed (resulting reduction of membrane thickness) due to developed transmembrane potential. This change of resistance is due to a small voltage buildup across the N02 with respect to $\mathrm{N} 2$, as the open switch resistance of SM is finite $\left(1 \times 10^{20}\right)$. This voltage caused SR1 and SR2 to open as the voltage exceeds $0.1 \mathrm{~V}$. The combined effect of open circuit resistance of $\mathrm{SM}$ and $\mathrm{R} 1, \mathrm{C} 1, \mathrm{R} 2, \mathrm{C} 2$ causes a small potential development across N04, resulting a small decrease of membrane resistance.

In the simulation shown in Figure 6.12, transmembrane potential settled at 0.995 $\mathrm{V}$ for an applied voltage of $10 \mathrm{~V}$. The steady state value of transmembrane resistance reached to $50 \mathrm{~K} \Omega$. In Figure 6.13 , a single pulse of $2 \mu \mathrm{s} 10 \mathrm{~V}$ was applied that 
resulted the same transmembrane potential and resistance. Also note that duration of EP can now be extracted from these simulation results as the time duration when the transmembrane resistance is settled. Other definition of the duration of EP can be applied (such as $50 \%$ change of resistance), however the resulted relationship among various cases as discussed in the next subsection will prevail. From these simulations, the duration of EP were $1.4 \mu \mathrm{s}$ for 1 pulse of $2 \mu \mathrm{s}$ duration, and $0.8 \mu \mathrm{s}$ for 2 pulses of $1 \mu \mathrm{s}$. Considering the fact that in both simulation cases, total excitation time was the same, it can be concluded that a single pulse results more EP compared to multiple pulses for the same total excitation time. To be able to compare such cases of EP quantitatively, two quantification terms are defined in the next subsection, along with summaries of simulation results.

\subsection{Degree of Electroporation and Electroporation}

\section{Fluid Exchange Parameter}

"Degree of Electroporation" term was referred previously to indicate the severity or extent of EP [137,138], but not defined yet. Here, we provide a definition of Degree of $\mathrm{EP}$ as the ratio of the total pore area that is formed during EP to the surface area of the cell. Using this definition, it clearly represents the extent of pore formation during the EP process and becomes an important criteria to distinguish among various cases of EP. This definition can be represented mathematically as follows,

$$
\text { Degree of Electroporation }=\frac{\text { Total pore area }}{\text { Cell surface area }} .
$$

Degree of EP represents a metric for denoting severity of EP. The significance of Degree of EP based on this definition are evident, such as:

1) For a given condition, the degrees of EP can be related with the magnitude of the applied potential.

2) Higher Degree of EP will allow higher amount of fluidic exchange through the 
cell membrane in a given amount of time in a certain buffer fluid.

3) The total fluidic exchange among various excitation conditions can be qualitatively compared by multiplying the degree of EP with the corresponding EP duration. We have termed this metric as "Electroporation Fluid Exchange Parameter" (EFEP) and defined as the product of Degree of EP and the duration of EP. EFEP would represent a metric to refer to the fluidic exchange resulted due to EP. Note that the duration of EP would be always smaller than the pulse duration as there is a certain time required for pore formation (nucleation period) and pore growth.

Degree of EP is unit-less, whereas EFEP has an unit of second. The steady state of transmembrane resistance enables us to calculate the total area of pores during EP. The higher the pore area, the higher the Degree of EP and the more fluid flow is expected per unit time. Using the expressions developed before, the total pore area is $n_{p} \pi r_{p}^{2}$ and the surface area of the cell is $4 \pi r_{a}^{2}$. Thus, Degree of EP $\left(\delta_{E P}\right)$ can be written with the help of Equation 6.9 as follows:

$$
\delta_{E P}=\frac{n_{p} \pi r_{p}^{2}}{4 \pi r_{a}^{2}}=\frac{\rho_{B} d_{m}}{4 \pi r_{a}^{2} R_{T M}}=\frac{K_{2}}{R_{T M}}
$$

where $K_{2}=\frac{\rho_{B} d_{m}}{4 \pi r_{a}^{2}}$ is proportionality constant for a specific cell type. Hence Degree of $\mathrm{EP}\left(\delta_{E P}\right)$ is inversely proportional to the total transmembrane resistance $\left(R_{T M}\right)$ due to membrane pores.

On the other hand, the metric EFEP (defined as $\xi_{E P}=\delta_{E P} *$ duration of $E P$ ) expresses a quantification that allows to compare various EP cases for effective fluidic exchange (such as, for drug delivery application). Higher value EFEP denotes probability of higher amount of fluidic exchange.

In Figure 6.15 and 6.16, Degree of EP and EFEP are plotted for several excitation conditions (cases). Simulation results with various types of excitations show that higher applied voltage results in reduced $R_{T M}$, hence increased $\delta_{E P}$ (Figure 6.15. However, pulse duration or number of pulses does not effect $\delta_{E P}$. On the other hand, simulation results of EFEP for the same excitation cases, as in Figure 6.15, demonstrated that EFEP is dependent on the pulse duration and number of pulses, 


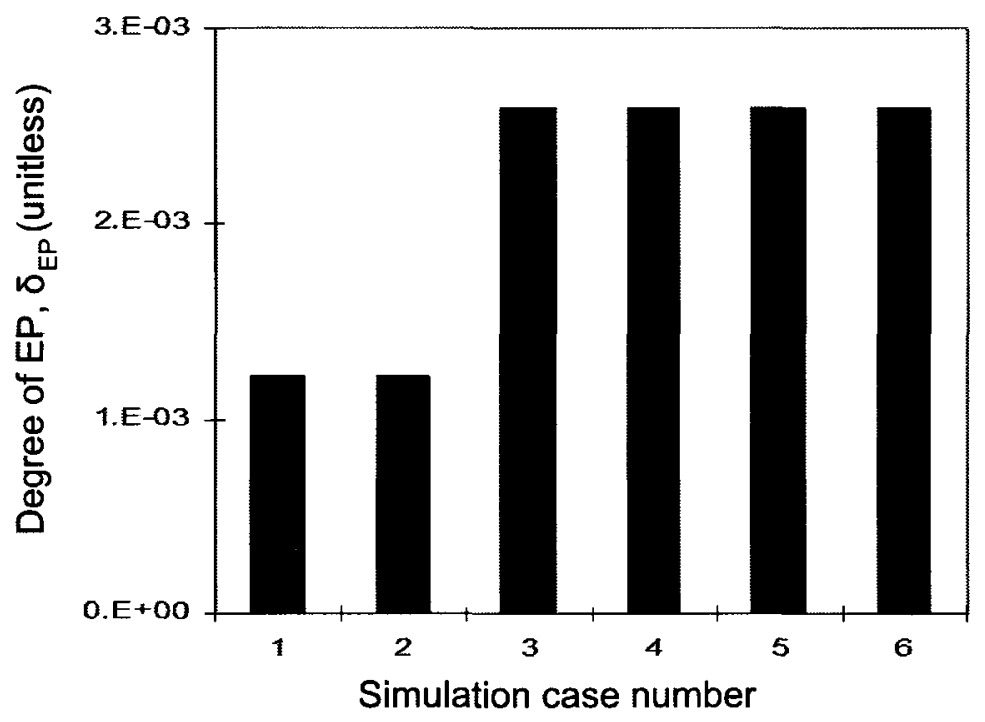

Figure 6.15 Plot of Degree of EP from various simulation cases are shown. The excitations for various simulation cases were: (1) $10 \mathrm{~V} 1 \mu \mathrm{s} 2$ pulses, (2) $10 \mathrm{~V} 2 \mu \mathrm{s} 1$ pulse, (3) $20 \mathrm{~V} 1 \mu \mathrm{s} 2$ pulses, (4) $20 \mathrm{~V} 2 \mu \mathrm{s} 1$ pulse, (5) $20 \mathrm{~V} 1$ $\mu \mathrm{s} 5$ pulses, and (6) $20 \mathrm{~V} 5 \mu \mathrm{s} 1$ pulse.

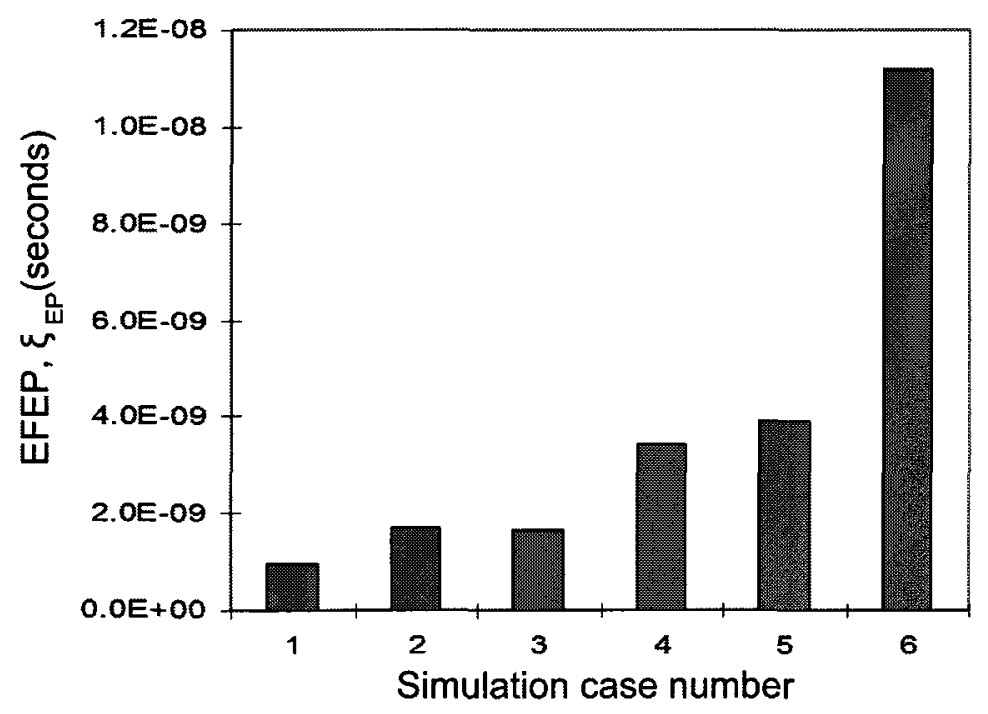

Figure 6.16 Plot of EFEP from various simulation cases are shown. The excitation cases were: (1) $10 \mathrm{~V} 1 \mu \mathrm{s} 2$ pulses, (2) $10 \mathrm{~V} 2 \mu \mathrm{s} 1$ pulse, (3) $20 \mathrm{~V}$ $1 \mu \mathrm{s} 2$ pulses, (4) $20 \mathrm{~V} 2 \mu \mathrm{s} 1$ pulse, (5) $20 \mathrm{~V} 1 \mu \mathrm{s} 5$ pulses, and (6) $20 \mathrm{~V} 5$ $\mu$ s 1 pulse. 


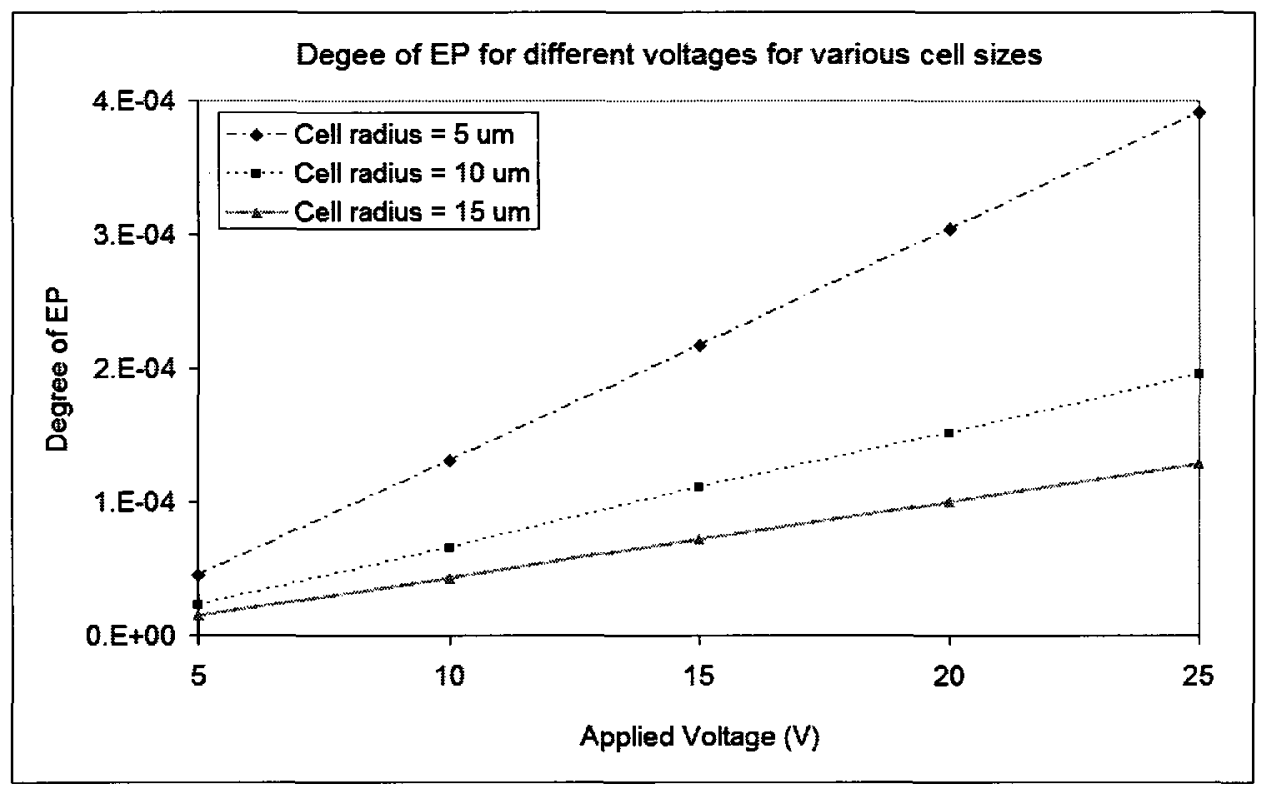

Figure 6.17 Plot of Degree of EP for a range of applied voltage is plotted from simulation results. Three different cell size results are shown.

as well as the applied voltage. All simulation pulse widths were $1 \mu \mathrm{s}$, unless otherwise mentioned. Minimum pulse width for these simulations must be larger than the nucleation period and the minimum pulse amplitude must be greater than that required for initiation of EP.

Comparing Case 1 and Case 2 in these figures, Degree of EP for both case are same, but higher EFEP resulted in Case 2 (175\%). Hence 1 pulse was more effective for higher EFEP compared to 1 pulses, delivering the same amount of energy. Same argument can be provided for Case 3 and Case 4 (203\%), as well as Case 5 and Case 6 (287\%). Note that the difference of EP for 5 pulses vs 1 pulse (Case 5 and Case 6 ) is prominent, which enhances the argument that a single pulse is more effective to achieve higher EFEP.

Comparing Case 2 and Case 3, it is evident that higher Degree of EP does not necessarily result higher EFEP, even for the same total excitation time. Here 1 pulse resulted higher EFEP with lower Degree of EP compared to 2 pulses with the same total excitation time and higher Degree of EP. 


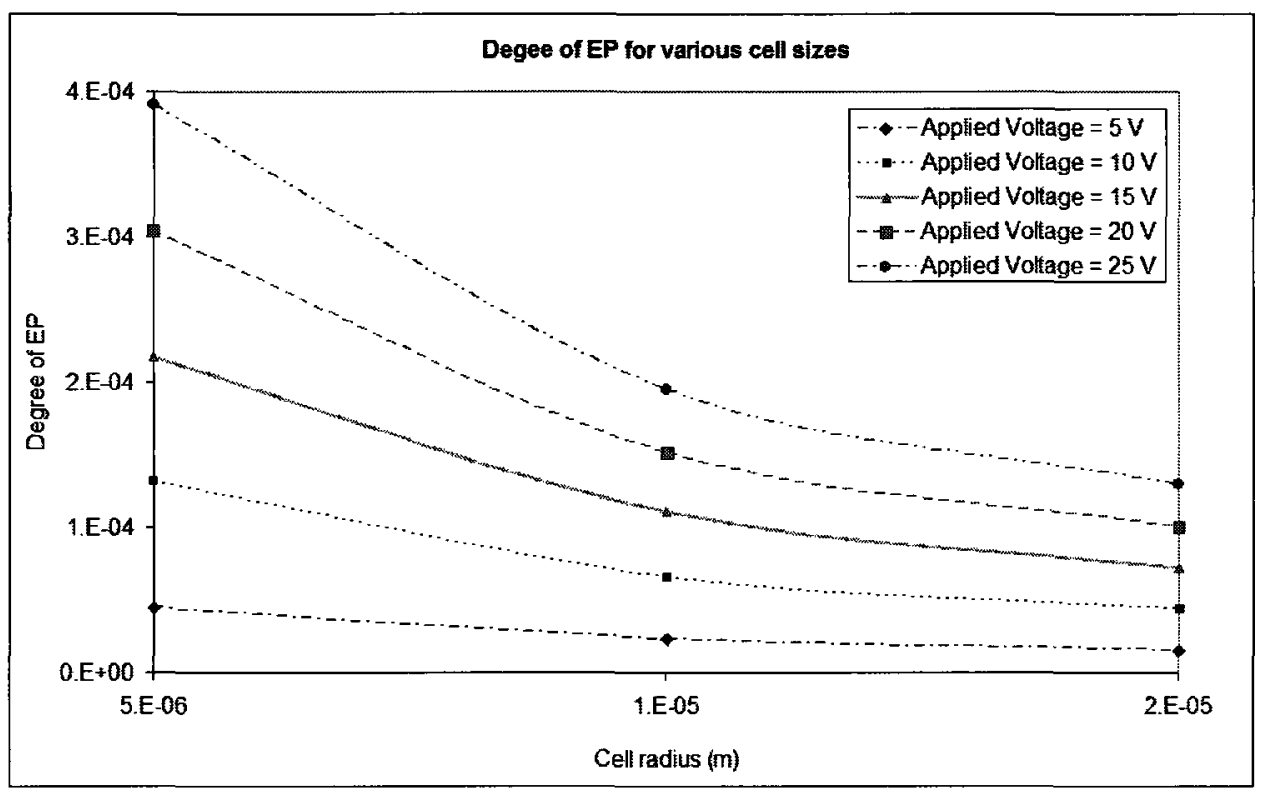

Figure 6.18 Plot of Degree of EP versus different cell diameters for various applied voltages.

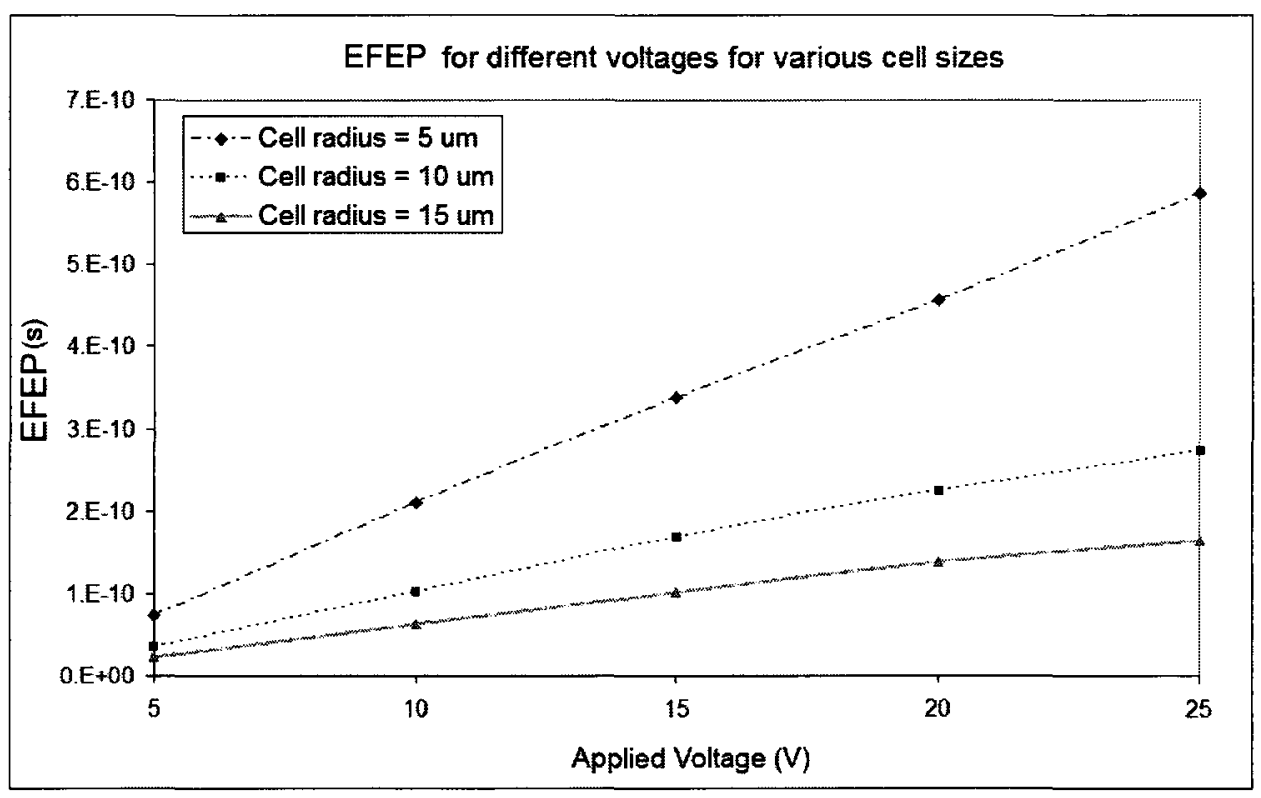

Figure 6.19 Plot of EFEP for a range of applied voltage plotted for different cell diameters. Pulse widths were $1 \mu \mathrm{s}$. 


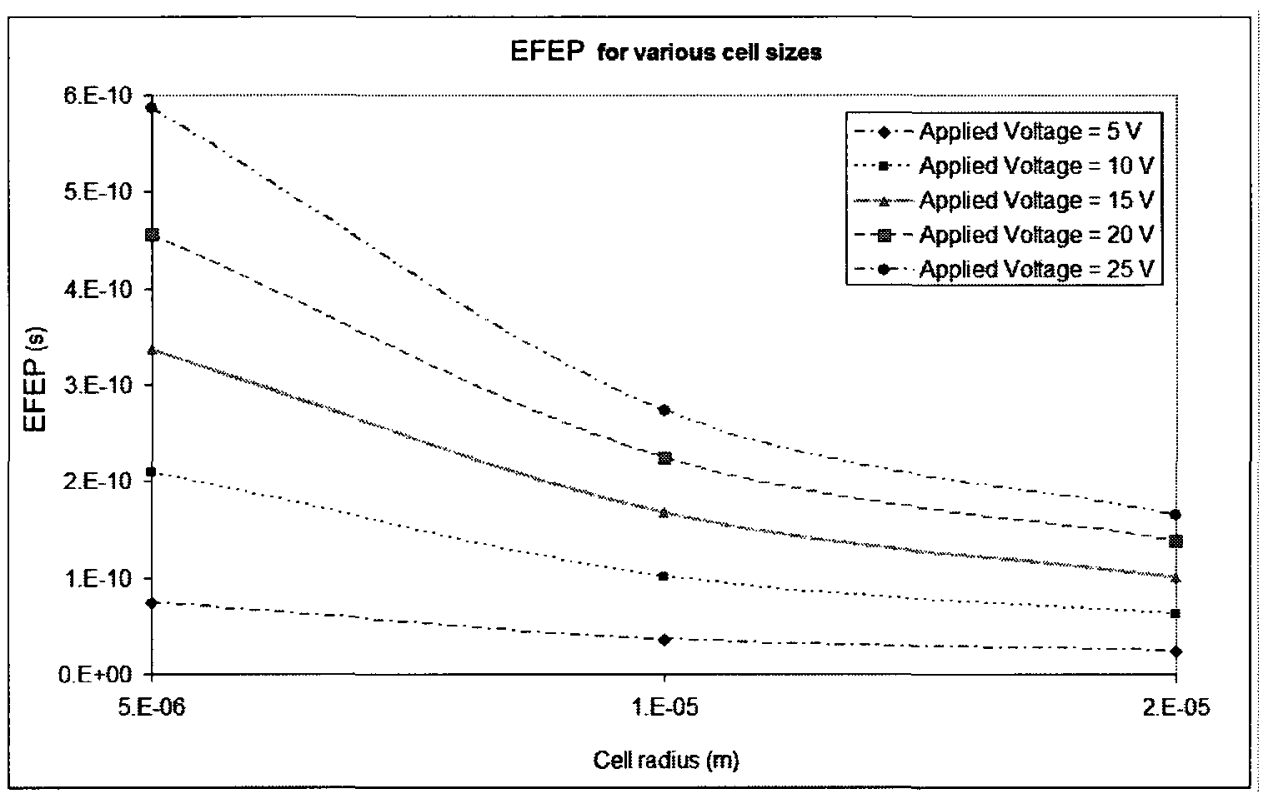

Figure 6.20 Plot of EFEP for different cell diameters with various excitation voltages. Pulse widths were $1 \mu \mathrm{s}$.

Figure 6.17 shows that Degree of EP is linearly related to the magnitude of the applied potential. Such simulation results agrees with experimental results previously reported $[137,138]$ and obtained with our final device. If the cell size increases, the same applied voltage causes less Degree of EP, as shown in 6.18. Figure 6.19 shows variation of EFEP for different applied voltage, where the relationship is not linear. The reason is that the duration of EP is not linear for various applied voltage (i.e. pore formation rate depends on the applied voltage). However, similar to Degree of EP, EFEP also reduces for larger cell size for any applied voltage. This relationship is shown in Figure 6.20, where a decrease of EFEP is observed for larger cell sizes.

In Figure 6.21 and Figure 6.22, Degree of EP and EFEP for various number of pulses are shown for a fixed applied voltage $(10 \mathrm{~V})$ and for three different total pulse duration. For each total pulse duration, 1, 2, 4, 5, 8 and 10 pulses were applied. Time between pulses were kept the same as the individual pulse duration. For example, to simulate $5 \mu$ s total pulse duration with 4 pulses, each pulse width was kept at $5 / 4=$ $1.25 \mu \mathrm{s}$ with gap between pulses also $1.25 \mu \mathrm{s}$. 


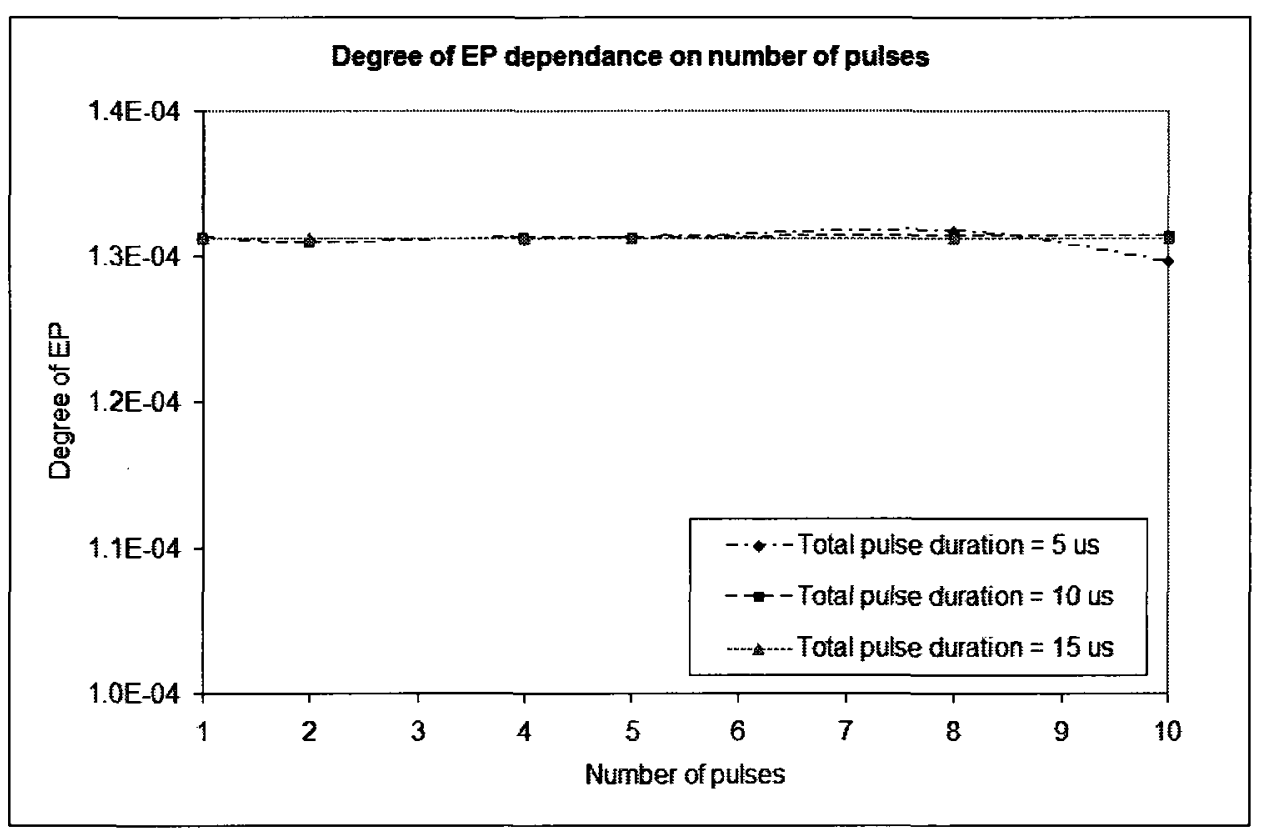

Figure 6.21 Plot of Degree of EP for various number of pulses with different total pulse durations. For multiple pulses, pulse gap was equal to pulse width.

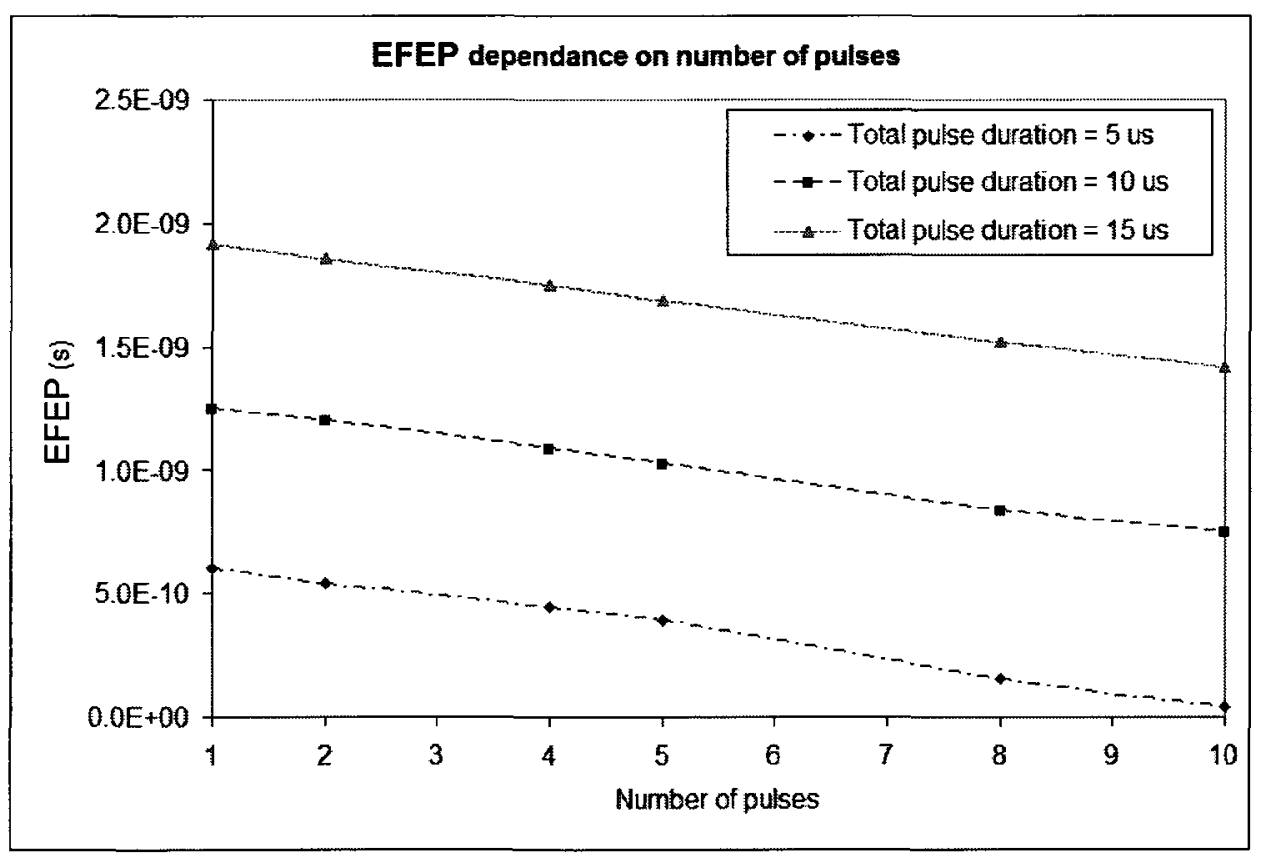

Figure 6.22 Plot of EFEP for various number of pulses delivering the same total energy with different total pulse durations. For multiple pulses, pulse gap was equal to pulse width. 
The simulation results show that Degree of EP remains constant irrespective to number of pulses or total pulse duration (i.e. various energy level with same applied potential). For very small pulse widths (such as, 10 pulses of $0.5 \mu$ s duration for each pulse) shows a small deviation due to comparable nucleation time to pulse width. For EFEP higher number of pulses results less EFEP. With increased total energy (i.e. higher total pulse width), EFEP increases.These simulation results show the same trend as our experimental results using the microfluidic device.

The proposed pore model can represent cell dynamics up to cell lysis phenomenon, after which cell structure becomes non-existent due to electrical lysis $[1,65,82,111]$. Degree of EP corresponding to the critical transmembrane potential can be termed as the "Critical Degree of EP". This Critical Degree of EP sustained for a critical time duration required for electrical lysis can be termed as "Critical EFEP". Below this Critical EFEP, the membrane pores will be reversible (resulting an EP phenomenon), whereas above this Critical EFEP, the membrane pores will become irreversible (resulting an EL phenomenon).

\subsection{Discussion}

Understanding from the extended model developed this chapter allows us to explain anomalies in experimental results with EP reported in last chapter. In Figure 5.36, there are following cases where more than one phenomenon was observed:

1. Applied voltage $=5 \mathrm{~V}$ and total time of excitation $=1 \mathrm{~ms}$ : (a) $\mathrm{EP}$ was observed for 1 pulse of 1 ms duration. (b) No effect was observed for 100 pulses of $10 \mu$ s duration.

2. Applied voltage $=20 \mathrm{~V}$ and total time of excitation $=10 \mu \mathrm{s}$ : (a) EP was observed for 1 pulse of $10 \mu$ s duration. (b) No effect was observed for 10 pulses of $1 \mu$ s duration.

Noting the fact that in all of these cases, 1 pulse delivering the complete energy resulted higher morphological change compared to the case where multiple pulses were 
delivered. From simulation results (Figure 6.16 and 6.22), we observed that a single pulse cause higher EFEP compared to multiple pulses when the total pulse duration is the same. A higher EFEP with a single would cause a detectable fluid flow, whereas a lower EFEP with multiple pulses might not cause a detectable fluid flow. For instance, in Case 1(a), the complete energy was delivered with 1 pulse of $1 \mathrm{~ms}$ duration and caused pores to form in cell membranes that was detected by stain reduction, whereas in Case 1(b), the energy was delivered in 100 pulses of $10 \mu$ s duration and did not cause detectable changes in stain (no effect). Similar arguments can be made for Case 2. These observations indicate that a single pulse will produce higher morphological changes compared to multiple pulses delivering the same amount of energy. This is particularly an important observation for EL, where we seek to inflict the maximum amount of morphological change with minimum amount of energy. Hence one single pulse for EL excitation should be preferred compared to multiple pulses for the same total pulse durations.

Furthermore, the analysis developed in this chapter can be related to the experimental results in term of Degree of EP and EFEP. Degree of EP is defined by the ratio of total pore area compared to the cell surface area. Hence, a higher Degree of EP would produce faster rate of stain reduction from a stained cell sample. This rate of reduction of stain can be found from the initial slope of Normalized Stain Reduction (NSR) in the plots of EP (Figure 5.37 to Figure 5.39). In ideal case of observation, the rate should be monitored while the pulses are still being applied, as this eliminates variability from dynamics of pore resealing process. However, in our experiments, the earliest data we have is at $5 \mathrm{~s}$. Table 6.2 calculated the slope of the curves at initial points from Figure 5.39 that shows higher slope is observed with higher applied potential. The increment of slope follows a linear trend that is in agreement with previous discussion (Figure 6.17).

For a fixed applied voltage, Degree of EP should not change. Data corresponding to Figure 5.37 and Figure 5.38, where all cases were subjected to the same applied voltages, are given in Table 6.3 and 6.4, respectively. For the case of applied voltage 
Table 6.2 Degree of EP and EFEP from experimental results (1 pulse of 10 ms duration)

\begin{tabular}{llll}
$\begin{array}{l}\text { Applied } \\
\text { potential }\end{array}$ & $\begin{array}{l}\text { NSR } \\
\text { at } \mathbf{5 ~ s}\end{array}$ & $\begin{array}{l}\text { Slope (reduction } \\
\text { of NSR/s) }\end{array}$ & $\begin{array}{l}\text { Reduction of NSR } \\
\text { at 145 s }\end{array}$ \\
\hline $5 \mathrm{~V}$ & 0.9891 & $2.18 \times 10^{-3}$ & 0.1497 \\
\hline $10 \mathrm{~V}$ & 0.9822 & $3.56 \times 10^{-3}$ & 0.2225 \\
\hline $15 \mathrm{~V}$ & 0.9617 & $7.66 \times 10^{-3}$ & 0.3251 \\
\hline $20 \mathrm{~V}$ & 0.9420 & $11.6 \times 10^{-3}$ & 0.422 \\
\hline
\end{tabular}

of $20 \mathrm{~V}$, the slopes calculated are very close to each other that indicates indifferent Degree of EP on number of pulses, in agreement with the simulation results given in Figure 6.21. However, for the case of applied voltage of $5 \mathrm{~V}$, the slopes do not follow the pattern. This disagreement in the case of $5 \mathrm{~V}$ applied voltage might result from either or combined effect of the following facts:

1) The low electric field might effect cell membrane protein pores to open rather forming hydrophilic EP pores.

2) Even if hydrophilic EP pores are formed, the pore size at these strength of electric field will be comparable to natural hydrophobic pores that exist in membrane proteins. Hence, a higher level of error is expected to be observed.

Note that the observations presented here used data at $5 \mathrm{~s}$, as no earlier data was available. A finer temporal resolution would more accurately resemble Degree of EP.

We can, as well, relate EFEP properties from the analysis to the experimental results. As EFEP is defined as Degree of EP times the duration of EP, higher energy pulses is expected to cause more EFEP. From the experimental curves, given in Figure 5.37 to Figure 5.39, a higher EFEP would result a higher reduction of stain at any certain time. To relate these to the observations, we have calculated the reduction of NSR at the latest data point $(145 \mathrm{~s})$ and included these data in Table 6.2 to Table 6.4. 
Table 6.3 Degree of EP and EFEP from experimental results (5 V applied potential)

\begin{tabular}{llll}
$\begin{array}{l}\text { Pulse } \\
\text { parameters }\end{array}$ & $\begin{array}{l}\text { NSR } \\
\text { at } \mathbf{5 ~} \mathbf{~}\end{array}$ & $\begin{array}{l}\text { Slope (reduction } \\
\text { of NSR/s) }\end{array}$ & $\begin{array}{l}\text { Reduction of NSR } \\
\text { at } \mathbf{1 4 5} \mathbf{~ s}\end{array}$ \\
\hline 1 pulse of $1 \mathrm{~ms}$ & 0.9951 & $0.98 \times 10^{-3}$ & 0.0661 \\
\hline 10 pulses of $1 \mathrm{~ms}$ & 0.9901 & $1.98 \times 10^{-3}$ & 0.1306 \\
\hline 25 pulses of $1 \mathrm{~ms}$ & 0.9793 & $4.14 \times 10^{-3}$ & 0.1904 \\
\hline
\end{tabular}

Table 6.4 Degree of EP and EFEP from experimental results (20 V applied potential)

\begin{tabular}{llll}
$\begin{array}{l}\text { Pulse } \\
\text { parameters }\end{array}$ & $\begin{array}{l}\text { NSR } \\
\text { at } \mathbf{5} \mathbf{~ s}\end{array}$ & $\begin{array}{l}\text { Slope (reduction } \\
\text { of NSR/s) }\end{array}$ & $\begin{array}{l}\text { Reduction of NSR } \\
\text { at } 145 \mathbf{~ s}\end{array}$ \\
\hline 100 pulses of $10 \mu \mathrm{s}$ & 0.9693 & $6.14 \times 10^{-3}$ & 0.1821 \\
\hline 1 pulse of $1 \mathrm{~ms}$ & 0.9638 & $7.27 \times 10^{-3}$ & 0.256 \\
\hline 10 pulses of $1 \mathrm{~ms}$ & 0.9538 & $9.24 \times 10^{-3}$ & 0.3295 \\
\hline 1 pulse of $10 \mathrm{~ms}$ & 0.9420 & $11.6 \times 10^{-3}$ & 0.422 \\
\hline 10 pulses of $10 \mathrm{~ms}$ & 0.9455 & $10.9 \times 10^{-3}$ & 0.6095 \\
\hline
\end{tabular}


From these data that correspond to EFEP, it can be easily noted that the maximum reduction of NSR occurred with application of $20 \mathrm{~V} 10$ pulses of $10 \mathrm{~ms}$ duration, delivering the maximum amount of energy within these excitation parameters. In addition, following observations are worthwhile to note from these data on EFEP that corresponds to previous modelling approach.

1) A higher applied voltage causes higher Degree of EP and EFEP, evident from experimental results (Table 6.2) that correspond to simulation results (Figure 6.17 and 6.19).

2) Simulation results show that a higher Degree of EP and EFEP results for smaller cell size. Thus EP or EL of smaller cells should require pulses with less energy compared to larger cell sizes.

3) A higher number of pulses cause more EFEP when higher energy is delivered from the experimental results as given in Table 6.3, but single pulse causes greater EFEP compared to multiples pulse as noted from the experimental results as shown in Table 6.4 row 1 and 2 or row 3 and 4 . These experimental results are consistent with simulation results as presented in Figure 6.22. These results of reduced EFEP with higher number of pulses delivering the same total excitation time is in agreement with the plots given in Figure 6.22.

Note that all numerical values calculated in this discussion would not correspond quantitatively to the cell model discussed earlier, but rather should be recognized qualitatively. This is because the cell model does not take into account of the internal organelles and statistical uncertainty that exists in nature. However, the model provides a comparative tool that can be utilized to analyze relative differences in EP or EL with various pulse parameters.

\subsection{Summary}

This chapter investigates and analyzes the electric circuit model of a cell membrane pore subjected to EP. Degree of EP and EFEP definitions enables us to compare effect 
of a single and multiple pulses. Degree of EP is directly dependent on the applied voltage whereas EFEP is additionally dependent on the pulse duration. A single pulse is found to be more effective to induce higher EFEP compared to multiple pulses delivering the same amount of energy. These results complement our experimental results discussed in the previous chapter. 


\section{Chapter 7}

\section{Conclusions and Future Directions}

This chapter summarizes key results from this research and provides future directions.

\subsection{Key Results}

Three devices were developed iteratively through this research with the aim of electrical lysis of biological cells to extract DNA from cell samples. The initial device fabricated using a microfluidic technology demonstrated feasibility of cell lysis phenomenon with a high electric field for rapid and on-chip operation, but required a high voltage power supply ( 750 to $900 \mathrm{~V}$ ) for excitation. Analytical expressions of electric fields developed inside microchannels were validated through Finite Element Method (FEM) modelling and analysis. The analytical expressions produced less than $10 \%$ error for microchannel length larger than $2.5 \mathrm{~mm}$. For smaller length of microchannels, an extension of the analytical expression was proposed. Moving cells through the microchannels using electrophoresis technique showed practical limitations due to cell adherence tendency with the channel surfaces. Electrical lysis phenomenon was demonstrated using the device by applying a high electric field. Rapid lysis (about $7 \mathrm{~s}$ ) and a low energy requirement (about $2.2 \mathrm{~J}$ ) rendered the microchannel based electrical approach a suitable method for DNA extraction from 
cells. However, requirement of a high voltage power supply lead us to develop further devices in an effort to reduce the applied voltage requirement.

Simulation of electric field of microchannel-based device has demonstrated several advantages of using such device to develop a high electric field. The electric field developed inside the microchannel was much higher compared to that inside the reservoirs. In addition, the current flow was limited by the cross sectional area of the microchannel as the microchannel is the only current path between the electrodes. Thus the power consumption was small and proportional to the volume of the microchannel.

The intermediate device contained integrated electrodes to reduce the voltage requirement. However, experiments produced limited results due to several other issues, like fluidic encapsulation, bubble formation, cell positioning etc. A protocol for fluidic encapsulation of bioMEMS device was developed.

The final device eliminated most issues and demonstrated ability of cell lysis and release of DNA from nucleus with the application of an excitation pulse of $20 \mathrm{~V} \mathrm{mag-}$ nitude and $5 \mathrm{~s}$ duration. In addition, electroporation phenomenon were observed by applying pulses of 5 to $20 \mathrm{~V}$ magnitude and $10 \mu \mathrm{s}$ to $100 \mathrm{~ms}$ durations. The electroporation phenomenon were quantified using image processing to evaluate reduction of stains after electroporation.

Thermal simulation showed that the upper bound of temperature rise of the final micro-device was limited to less than $2.2^{\circ} \mathrm{C}$ for a $20 \mathrm{~V}$ excitation for $5 \mathrm{~s}$. This temperature rise due to device excitation using electric pulse was low and should not influence any significant change of cell morphology. Electric field simulation demonstrated that an electric field of about $2 \mathrm{MV} / \mathrm{m}$ was developed by the application of $20 \mathrm{~V}$ through the interdigitated electrodes. This electric field strength was higher than the threshold electric field to cause electroporation and electrical lysis to release DNA.

We also proposed an improvement of the current electric circuit model to analyze electroporation through modelling of cell membrane pores. Such analysis enabled us 
to define two quantification terms for electroporation: (a) Degree of Electroporation and (b) Electroporation Fluid Exchange Parameter (EFEP). These quantification terms allowed comparison of electroporation with various pulse excitation, eg. a single pulse compared to multiple pulses. It was shown through simulation and experimental results that Degree of Electroporation was only dependent on the applied voltage, whereas EFEP was additionally dependent on the pulse duration and number of pulses. Through analysis and experimental results, it was demonstrated that (a) higher voltage caused a higher Degree of Electroporation, (b) multiple pulses resulted higher EFEP when increased energy was delivered, and (c) a single pulse was more effective to induce higher EFEP compared to multiple pulses when the same total excitation times were compared.

\subsection{Comparison with other devices}

Table 7.1 compares the features of the final device with other similar devices reported in literature. This work was the first to use interdigitated electrode configuration that provides an area coverage of up to $50 \%$, which was higher than any other possible configurations. Furthermore, the electrode at the bottom of the microchannels compared to that in sidewall of microchannels enabled use of larger sized cells as samples relative to the electrode gaps, in addition to smaller cells. Thus our device was the first to test large cells like cheek cells. A longer pulse width was required in our device, which might be due to a larger cell size, stronger cell wall, and existence of internal cell structures such as presence of nucleus and cytoskeleton.

One of the major limitation of the device was degradation of the electrodes after several experiments that led to a non-functioning device due to cracks or lift off of electrodes. This is attributed to the reaction of binding metals with the buffer fluid and can potentially be improved by isolating the layer using non-reactive material or replacing the binding material to other, more inert material. 


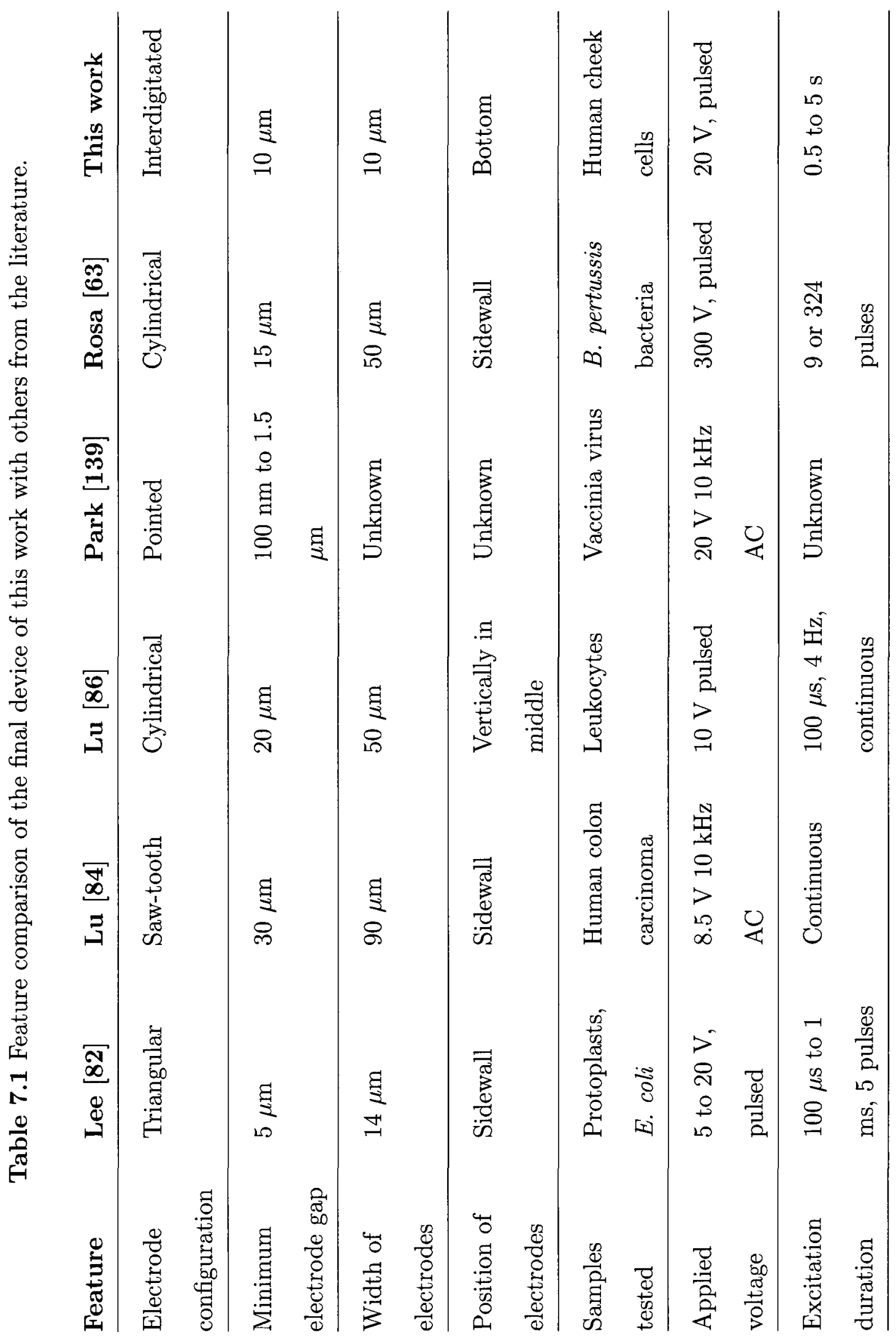




\section{3 $\quad$ Future research directions}

This research clearly demonstrated the feasibility of applying electrical lysis method for extraction of DNA very quickly from cell samples. The developed device was rapid and portable, as well as integrate-able with other components of DNA detection process. The findings from this research can be utilized to develop into a complete commercialize-able product that integrates multiple steps of DNA processing onto a single chip, such as DNA purification device or complete DNA detection device. As on-chip PCR amplification and on-chip DNA detection were demonstrated by various researchers which use compatible device platform [30, 41, 42, 44, 46, 48, 48, 52], the electrical lysis method can now be easily integrated with such processing steps for DNA detection for a complete system. Such concepts are being investigated as a future development at Medical Devices Center of Ottawa Heart Institute by Dr. Mussivand's research group.

Rapid and portable on-chip low-voltage electroporation device can be utilized for clinical drug and gene delivery in vitro (stem cell, plasmid etc.) or in vivo (through epithelial tissue). Micro robot devices with on-board battery and propelling mechanism can use the low voltage electroporation technique to deliver drugs to specific sites in human body for cancer treatment etc. Furthermore a combined electroporation and electrical lysis device might find use in some biochemistry laboratories. 


\section{Appendix A}

\section{Device Designs}

This chapter provides color plate device layout of the devices developed during this research. The initial device was developed using Protolyne Microfluidic Technology, the intermediate device was developed using Metal Multi-User MEMS Processes (MetalMUMPs), and the final device was developed using SensoNit Fabrication Technology.

\section{A.1 The Initial Device}

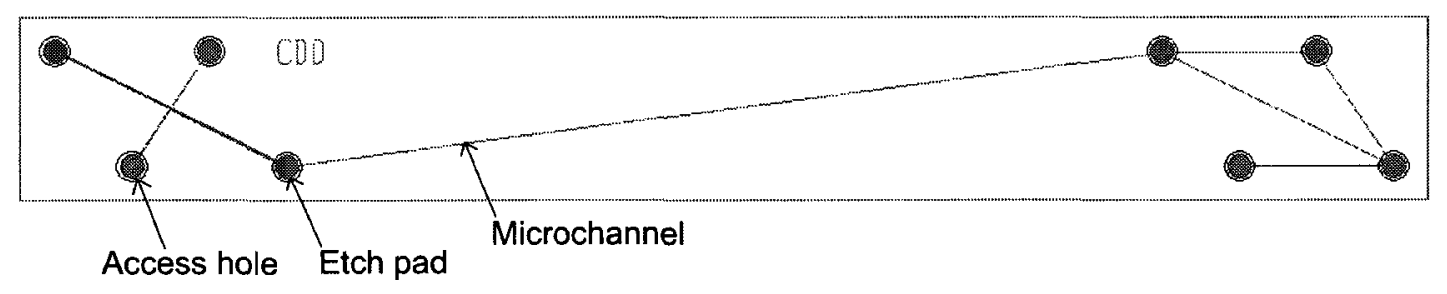

Figure A.1 Full layout of the initial device. 


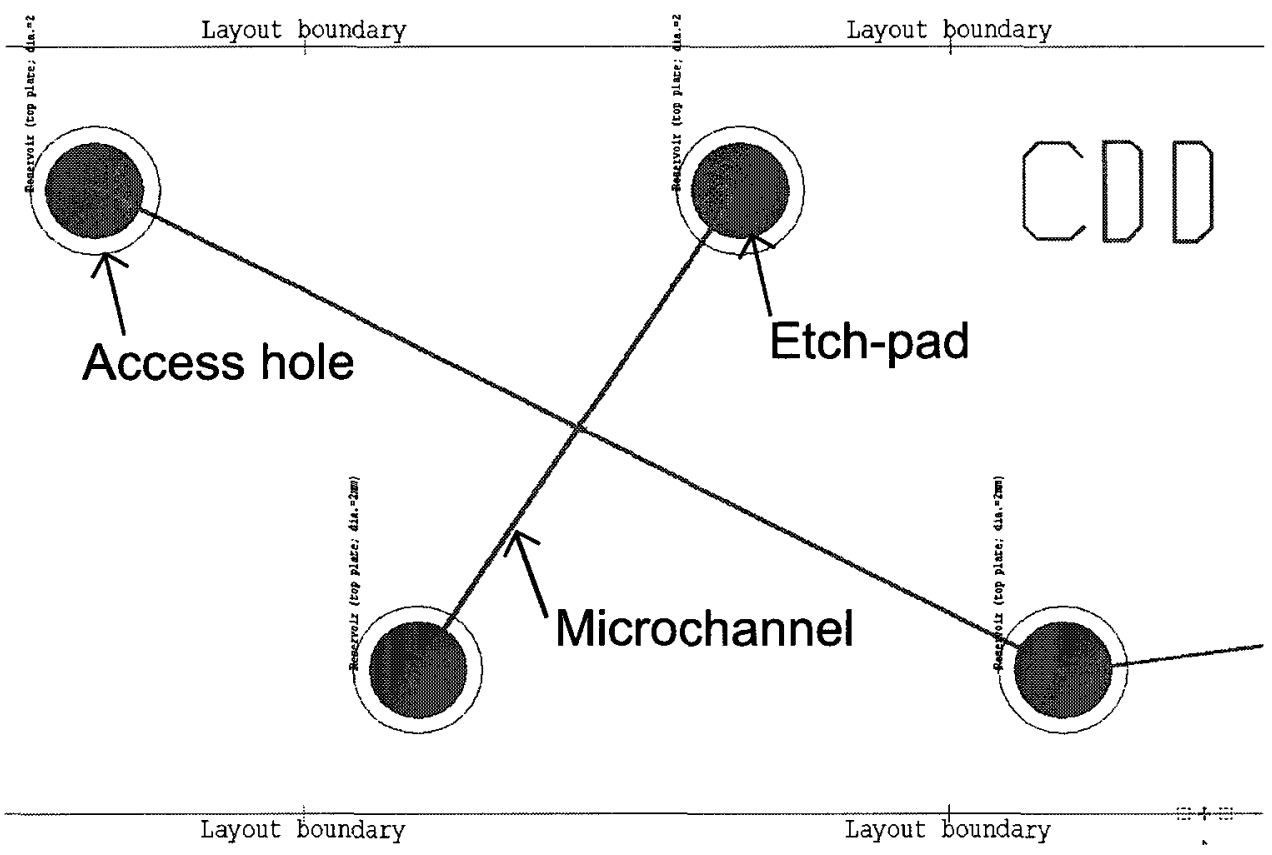

Figure A.2 Left hand side section of the initial device is enlarged to show details of the layouts of the microchannels and reservoirs.

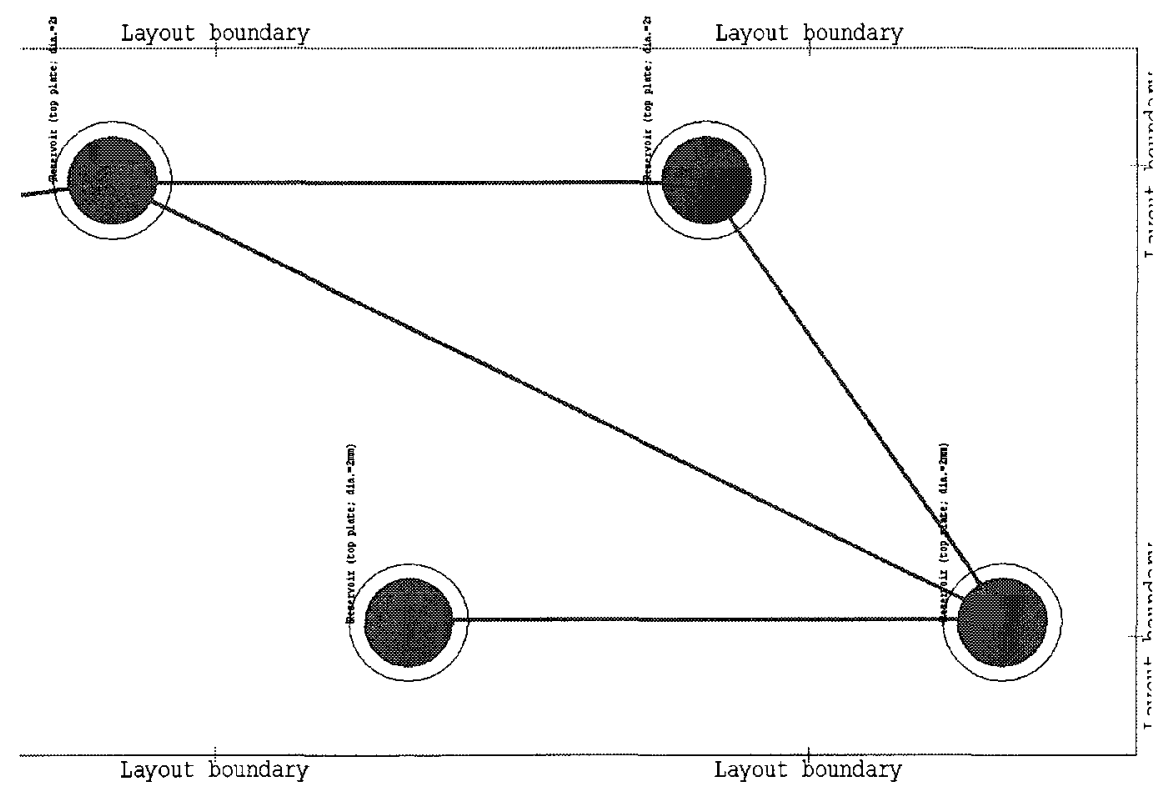

Figure A.3 Right hand side section of the initial device is enlarged to show details of the layouts of the microchannels and reservoirs. 


\section{A.2 The Intermediate Device}




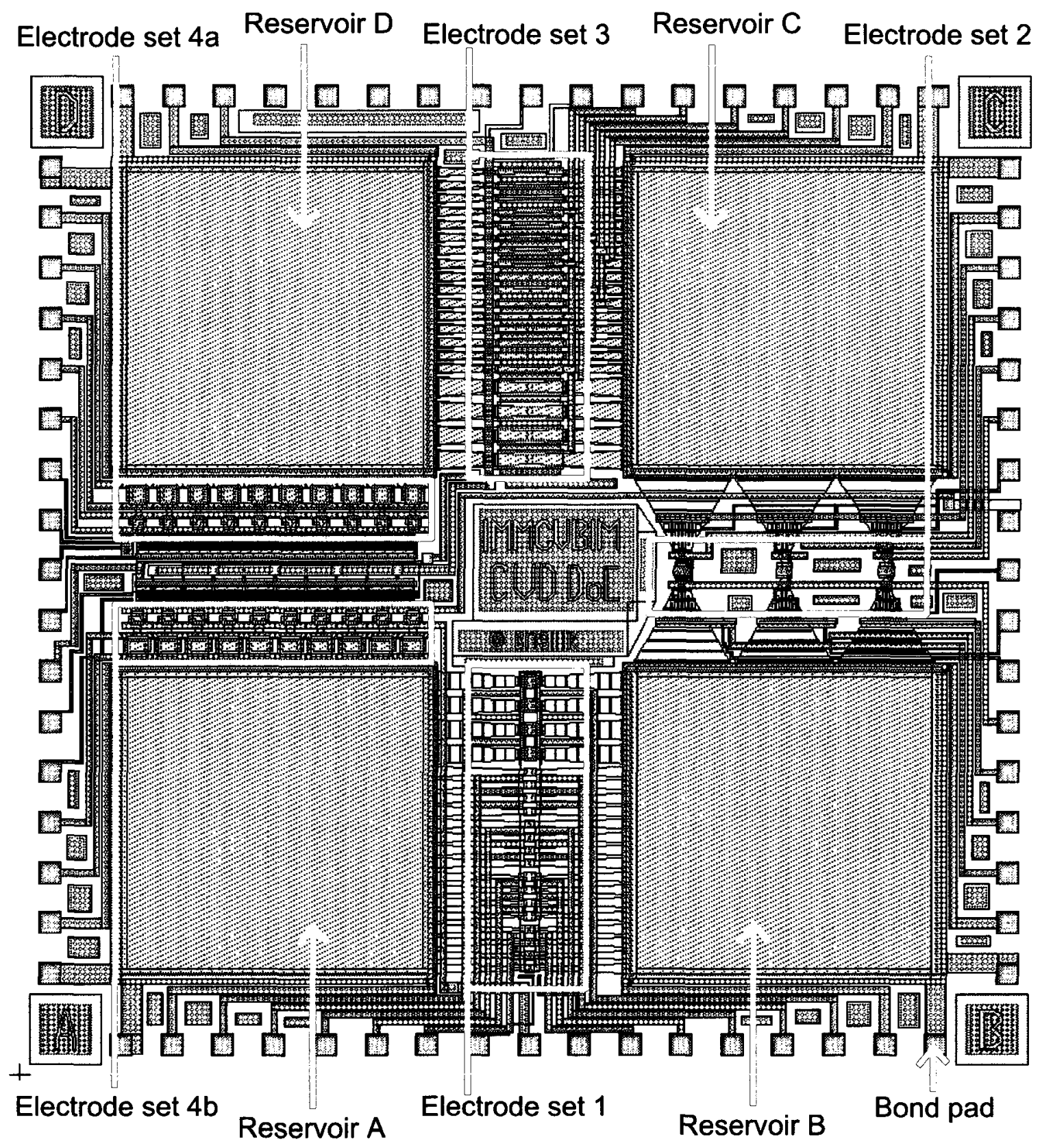

Layers: $\square$ OXIDE1 $\square$ POLY $\square$ NITRHOLE 四METANCH $\square$ GOLDOVP $\square$ METAL

Figure A.4 Layout of the complete MetalMUMPs chip showing different important elements of the design. Mask layer legends is shown at the bottom of the layout. 


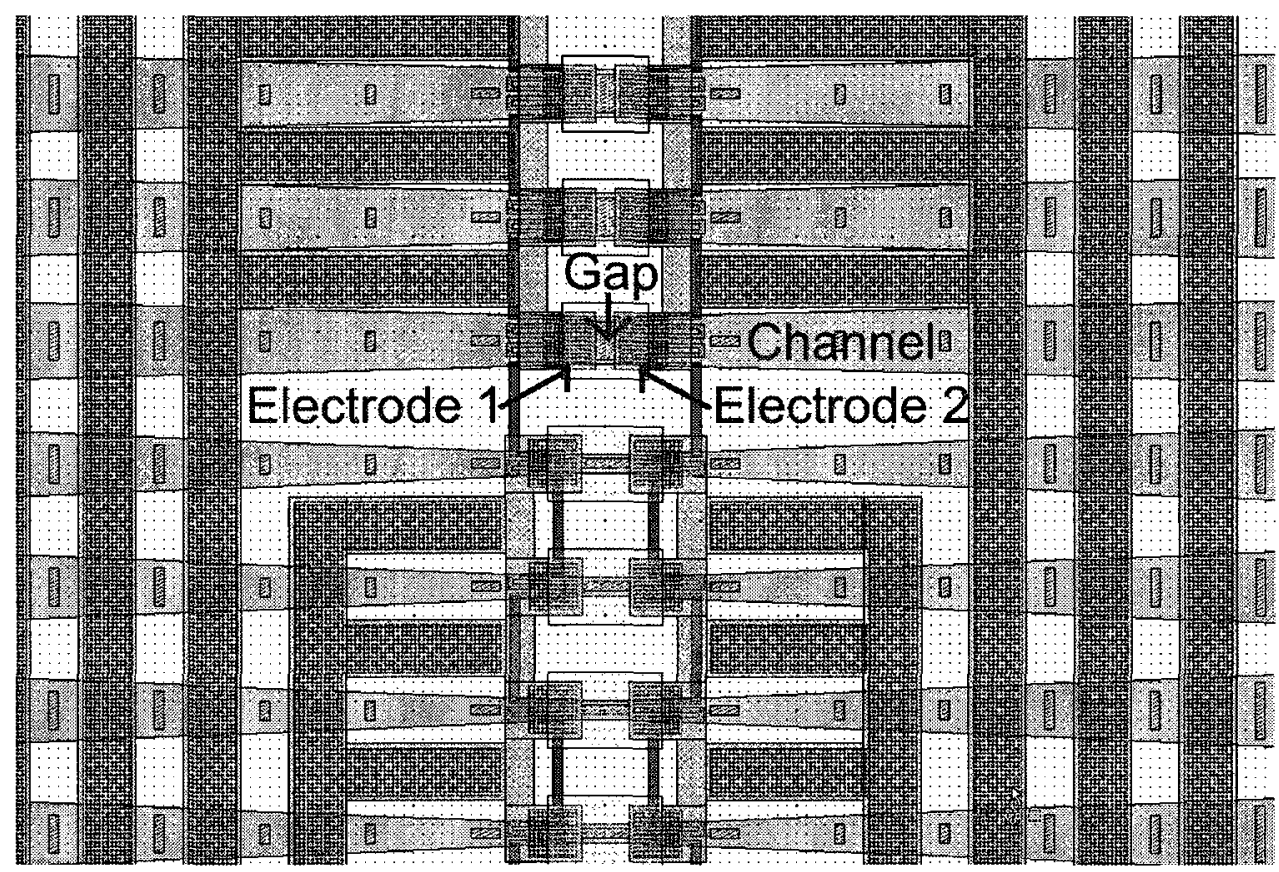

Figure A.5 Layout of a section of Electrode Set 1 in the chip.

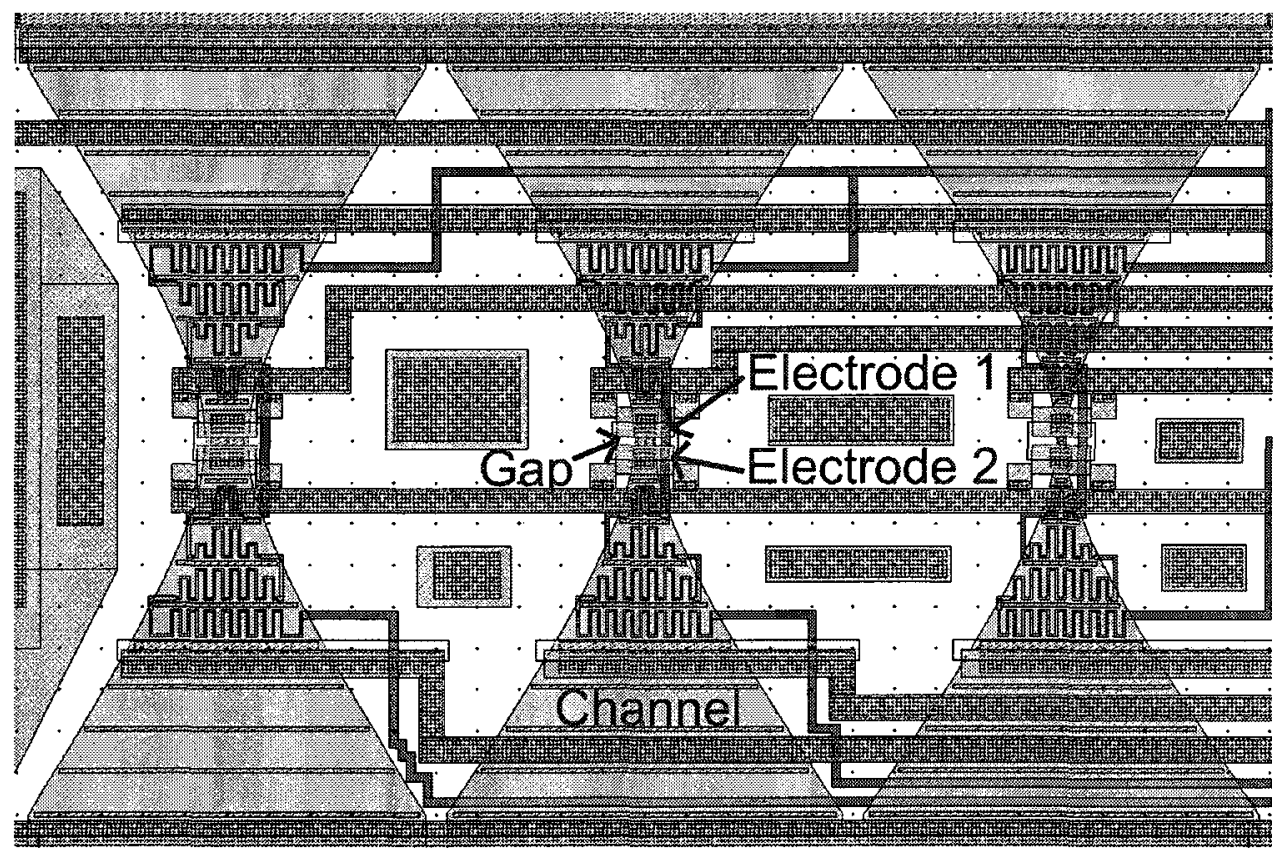

Figure A.6 Layout of Electrode Set 2 in the chip. 


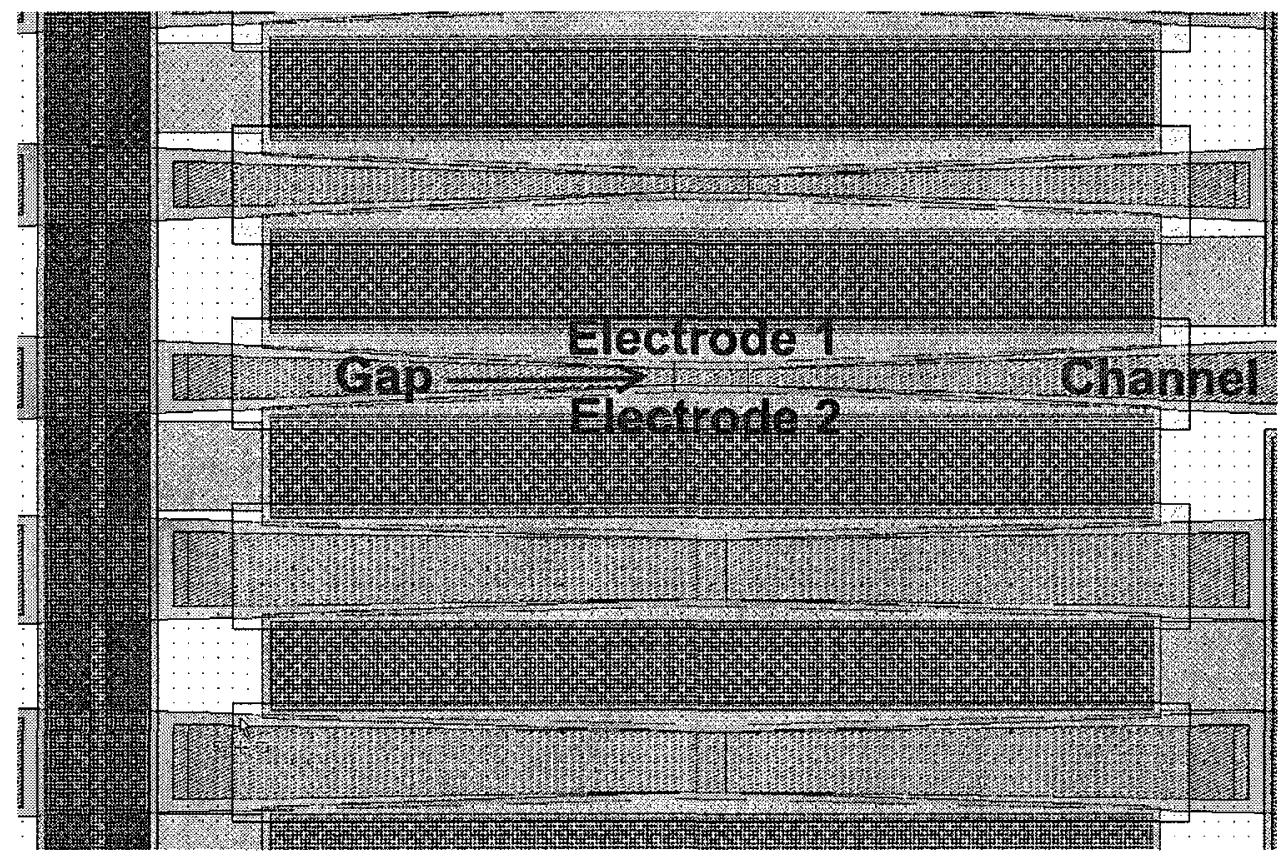

Figure A.7 Layout of a section of Electrode Set 3 in the chip.

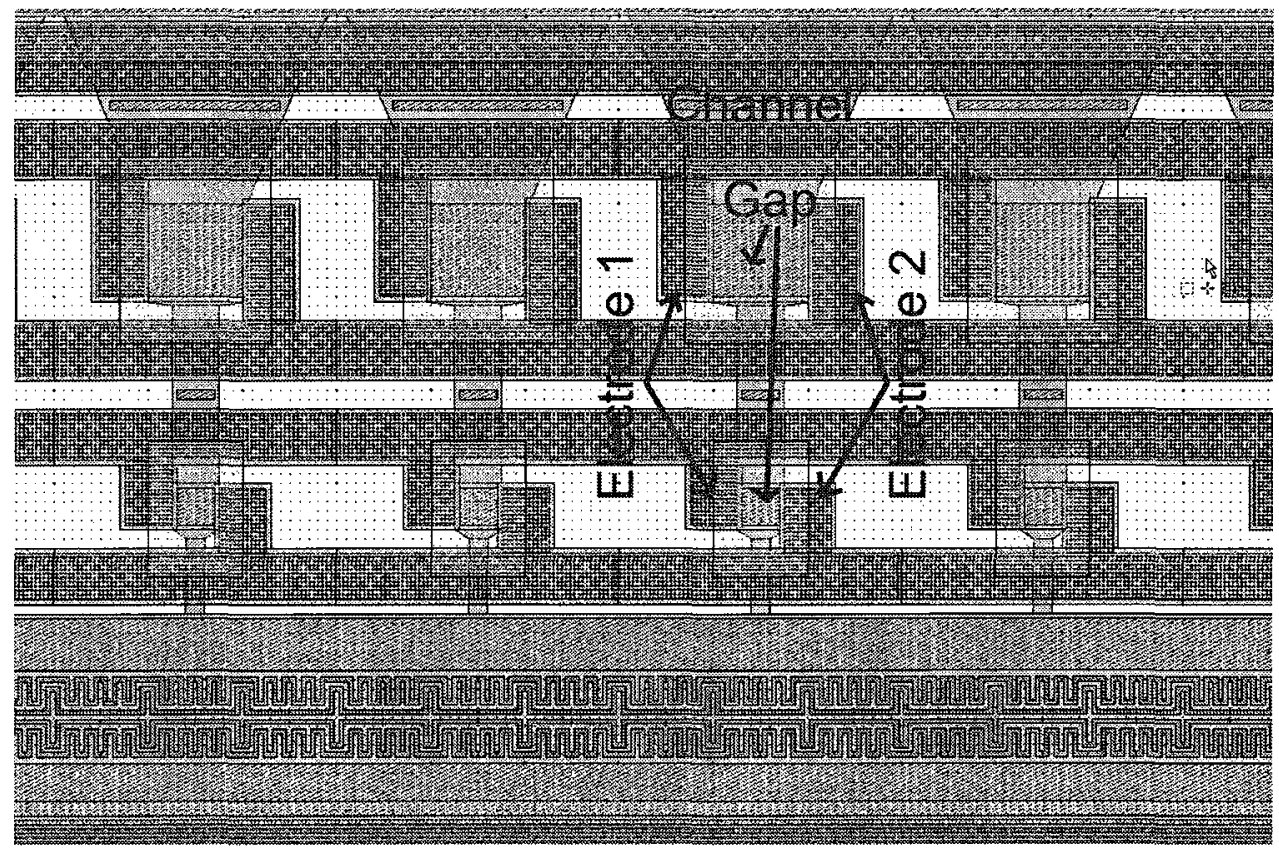

Figure A.8 Layout of a section of Electrode Set 4a in the chip. 


\section{A.3 The Final Device}




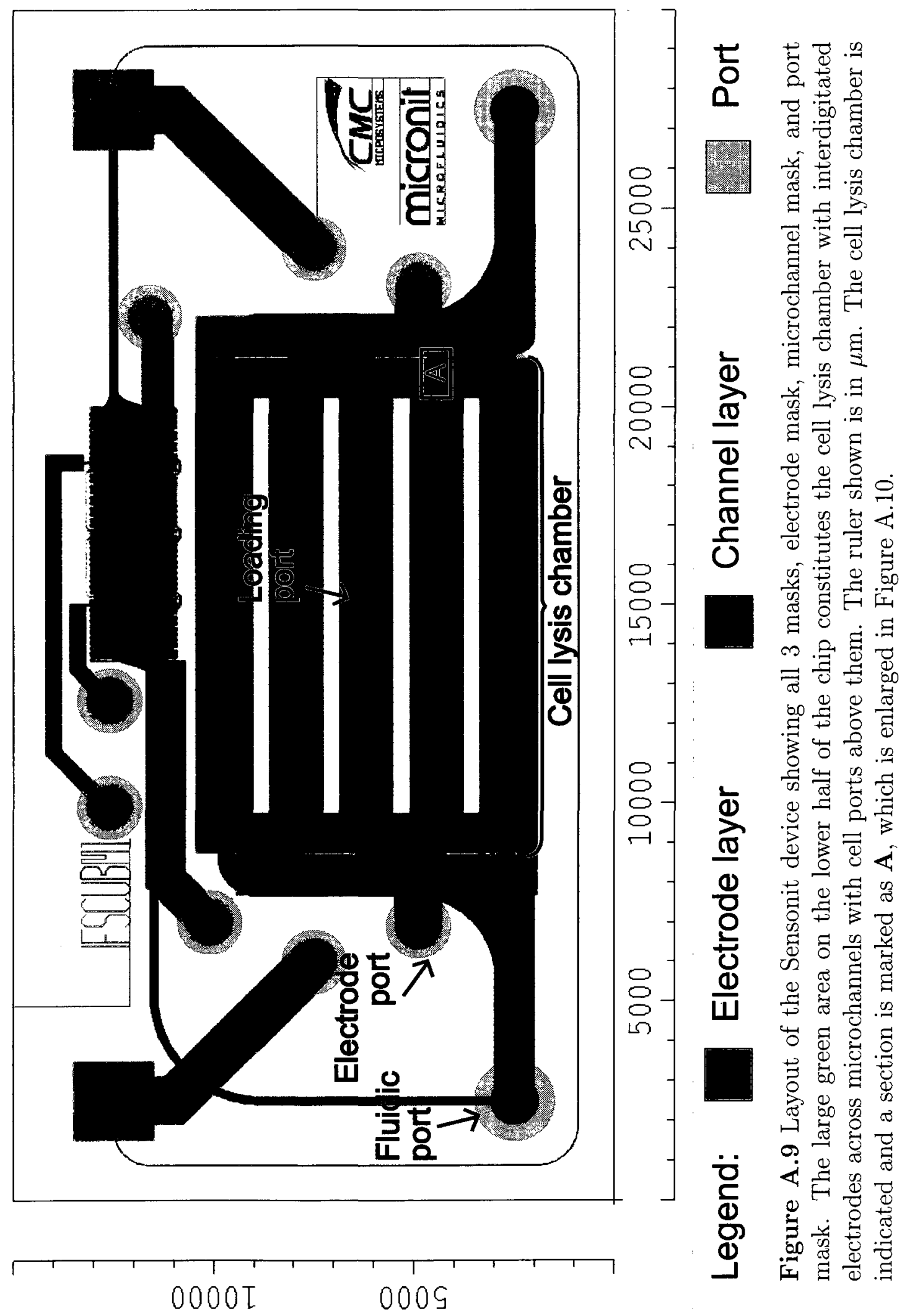




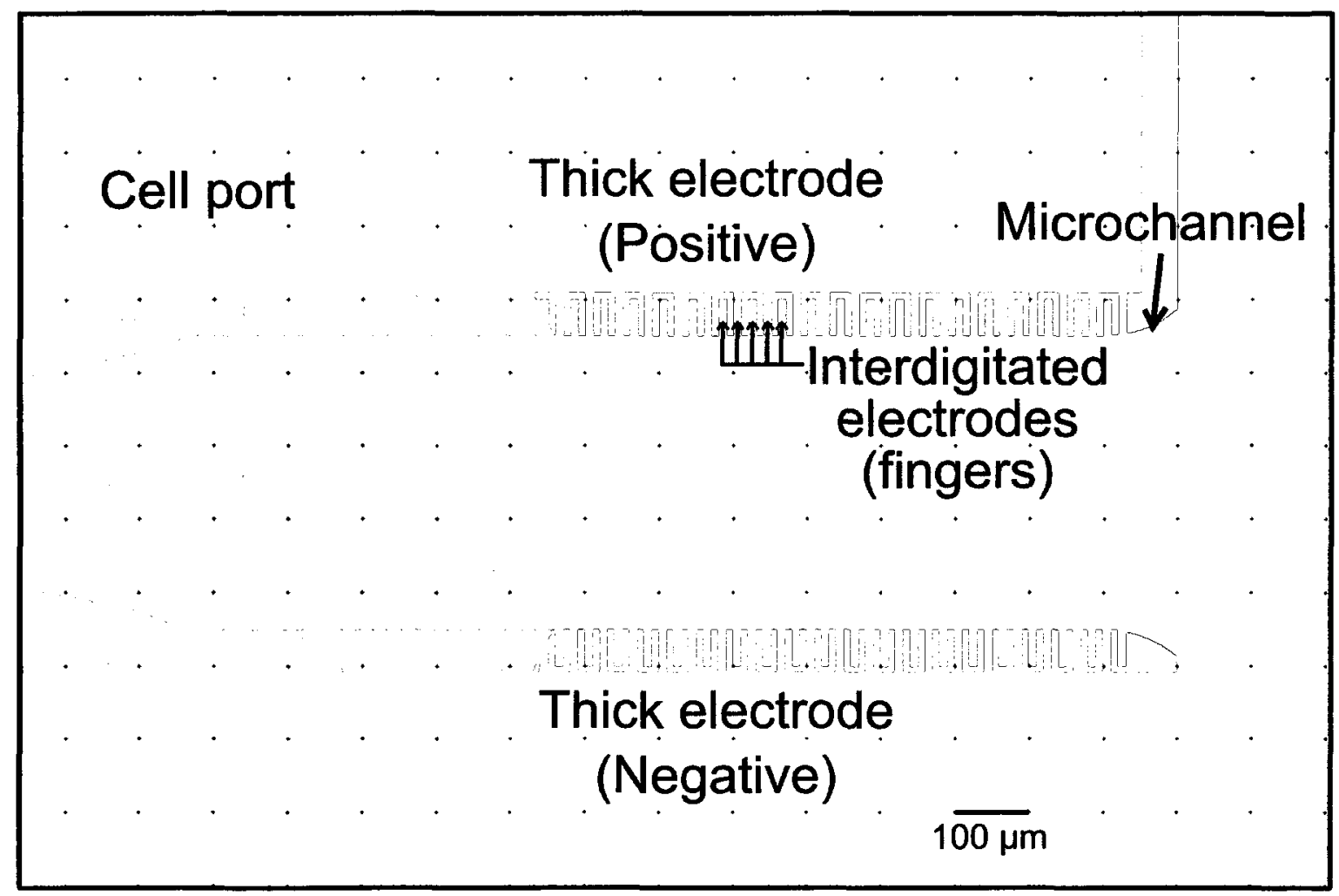

Figure A.10 An enlarged version of the layout of section $\mathbf{A}$, showing portion of the cell lysis chamber (the section marked as $\mathbf{A}$ in Figure A.9). A cell port, some interdigitated electrodes (fingers) on a microchannel and two thick electrodes are indicated. A scale bar is shown at the bottom. 


\section{Appendix B}

\section{Protocols}

All protocols related to this thesis work are listed in this chapter. The protocols that were developed through this research are denoted by the term "developed".

\section{B.1 Developed protocol for fluidic encapsulation}

\section{Components needed:}

1. 4 sticky-note clean-room paper.

2. 1 clean-room tissue.

3. A metal block (machine-cut) with a footprint (bottom area) slightly smaller than the area to be exposed. A footprint of $8.5 \mathrm{~mm} \times 8.5 \mathrm{~mm}$ was chosen for our device, as a clearance of $0.25 \mathrm{~mm}$ on each side produced optimal results.

4. Some polyimide (about $1 \mathrm{~mL}$ ).

5. Some distilled water (about $1 \mathrm{~mL}$ ).

\section{Tools required:}

1. A general purpose cutting blade.

2. A programmable heater.

3. A flat-head screwdriver (or tweezers).

The components and tools needed for this procedure is shown in Figure B.1.

Brief protocol:

1. A clean-room stick-on paper and a clean-room tissue were attached together so that the sticky side of the paper attaches with the tissue. They were aligned such that one corner of the sticky side of the paper perfectly matches with a corner of the tissue. 


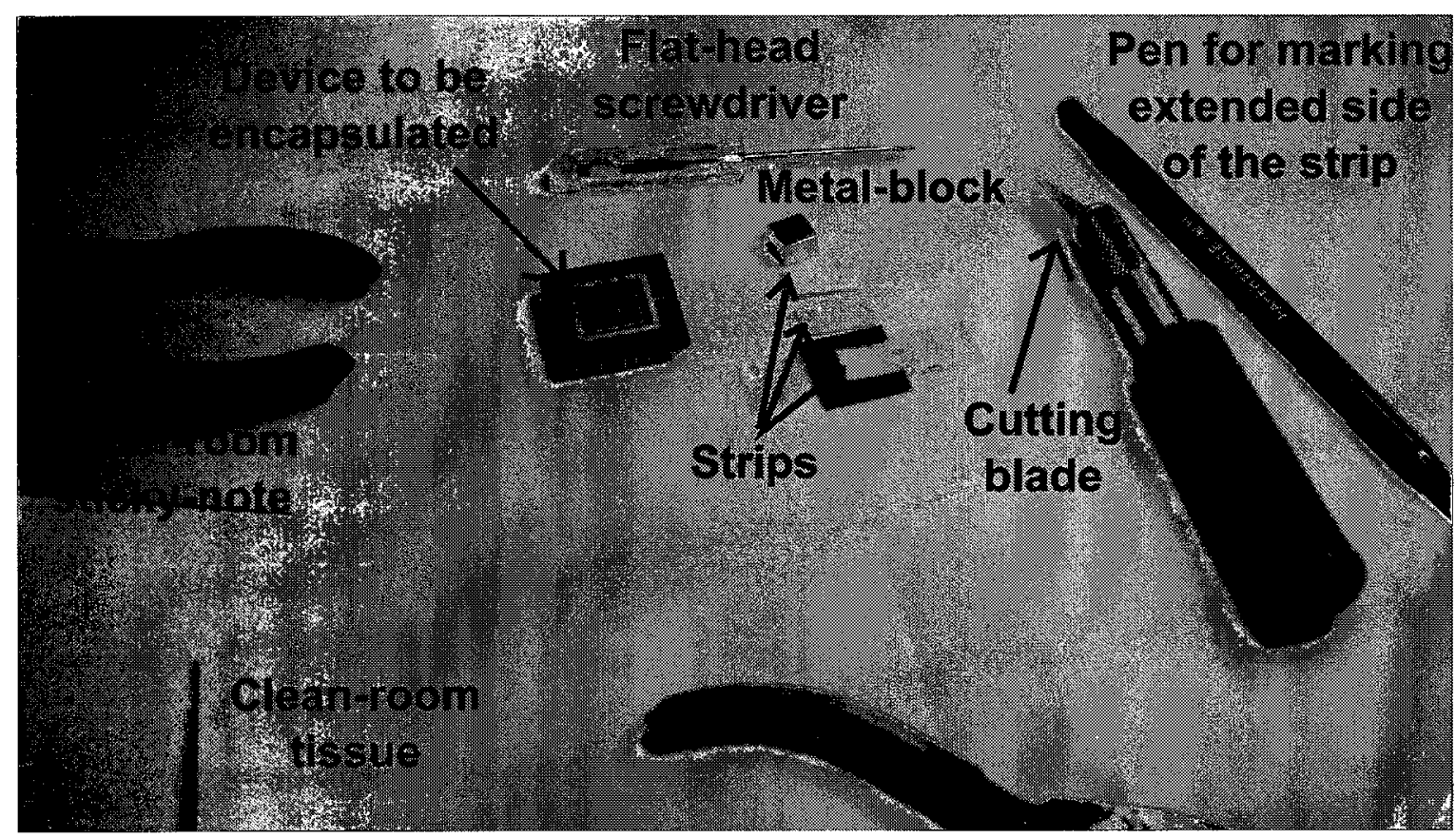

Figure B.1 A photograph showing the components and tools needed for the developed fluidic encapsulation protocol.

2. Place the machine-cut metal-block on top of the paper-tissue combination with tissue side on top, so that one corner of the footprint (bottom area) of the block perfectly aligns with the aligned paper-tissue corner.

3. Use the general purpose cutting blade to cut the paper-tissue combination along one of the remaining edges of the metal block (edge parallel to the tissue fibre direction is recommended). Continue cutting along the line past the metal block to have an extension and cut the remaining side of the strip. Mark the extended side with a pen, so that it can be readily identified at a later time.

4. Repeat the procedure 2 to 3 to prepare four identical strips, one for each side of the encapsulation area.

5. Apply a drop of distilled water on the paper side of the strip to make the surface wet. Remove excess water by shaking the strip.

6. Place the strip on the chip so that the paper side faces down and the aligned edge matches with one edge of the region to be covered with polyimide. Use a thumb to gently hold it in place while a flat screw-driver (or tweezers) to adjust this matching.

7. After properly positioning the strip, while still held by a thumb in the middle, apply a small amount of polyimide along the edge of interest. Optimal results 
were obtained by dropping a minimal amount of polyimide along the edge of interest of the strip. Use the flat-head screwdriver to evenly spread-out the polyimide as required. Polyimide holds the strip in place. The thumb can be released at this step.

8. After polyimide settles, use the flat-head screwdriver (or tweezers) to lift the extended side of the strip upwards so that the strip becomes vertical on the device with the edge of interest touching the device. Now lift the strip up vertically and discard.

9. Tilt the device about $30^{\circ}$ such that polyimide flow outwards from the edge of interest. Allow couple of minutes for polyimide to resettle. Then place the device on the hotplate.

10. Set the programmable hot-plate heater to heat the device from room temp to $120^{\circ} \mathrm{C}$ (with a ramp of $10^{\circ} \mathrm{C} / \mathrm{min}$.) and allow it to be heated for 15 mins, then turn off heater to cool off the device to room temp. After ensuring that the device temperature is at room temperature, remove it from the hotplate. [Caution: touching the device when it is hot might cause injury.]

11. Repeat steps (5 to 10) for all four sides to be encapsulated.

12. After all four sides are complete, perform a final cure step using the hot plate to heat the device up to $180^{\circ} \mathrm{C}$ for 5 mins.

13. Turn off the heater and leave the device to cool off to room temperature. Observe under a microscope to verify polyimide encapsulation.

\section{B.2 Developed protocol for staining free-flowing cells}

Reagents required for each sample:

- Harris Alum Hematoxylin $(20 \mu \mathrm{l})$.

- Eosin Y Alcohol $(20 \mu \mathrm{l})$.

- D- H2O (at least $15 \mathrm{ml}$ ).

- Hypotonic solution (1 ml).

The developed protocol is given below:

1. Collect sample cells (cheek cells) by rubbing two sterile buccal swabs (one at a time) against inner wall of cheek inside mouth for at least 2 mins. 
2. Wash the swabs with $1.5 \mathrm{ml}$ Deionized Water $\left(\mathrm{D}-\mathrm{H}_{2} \mathrm{O}\right)$ into two Eppendorf tubes.

3. Centrifuge the tubes at a low speed (2000 rpm) for $5 \mathrm{~min}$.

4. Discard supernatant from the tubes, leaving $0.5 \mathrm{ml}$ solution.

5. Add $20 \mu \mathrm{L}$ of Hematoxylin in the tubes and wait for 10 mins.

6. Add $1 \mathrm{~mL} \mathrm{D}-\mathrm{H}_{2} \mathrm{O}$ in the tubes and re-suspend cells.

7. Centrifuge the tubes at low speed $(2000 \mathrm{rpm})$ for $2 \mathrm{~min}$.

8. Discard supernatant, leaving about $0.5 \mathrm{~mL}$ liquid.

9. Perform the last 3 steps 2 more times.

10. Now, add $20 \mu \mathrm{L}$ of Eosin in both tubes and wait for 2 mins.

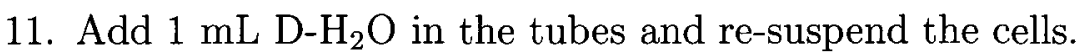

12. Centrifuge the tubes at low speed $(2000 \mathrm{rpm})$ for $2 \mathrm{~min}$.

13. Discard supernatant, leaving about $0.5 \mathrm{~mL}$ solution.

14. Perform the last 3 steps 2 more times.

15. Store the tubes in a cool, dry and dark place and use within a week. The solutions in tubes are the source of stained sample cells in our experiments.

Just before experiments, add $1 \mathrm{ml}$ of hypotonic solution in each tube, and inject in the device as soon as possible (the hypotonic solution takes at least 10 mins to disintegrate cell membranes without any external excitation)

\section{B.3 Protocol for fixing nanoports on top of fluid ports}

1. Hold the port-base upside down and place the O-ring in the groove.

2. Position an adhesive ring around a fluidic port of the device, where the port-base is to be fixed.

3. Flip the port-base and quickly place it (with the O-ring) on top of the adhesive ring, so that the port-base and the adhesive ring are aligned.

4. Secure the setup with a paper clip by griping the port-base with the device.

5. Carefully adjust the position of the port-base as needed for proper alignment. 
6. Place the setup inside an oven preheated at $180^{\circ} \mathrm{C}$, and wait for 1 hour.

7. Turn the oven off and remove the device from the oven using tweezers and place it on a safe place to cool off to room temperature. (Caution: The device is hot when removed from the oven.)

8. Verify proper positioning and bonding of the port-base with the device.

\section{B.4 Protocol for fixing metal electrodes through electrode ports}

1. Mix small amounts of the two components (ratio of components $=1: 1$ ) of the Silver Conductive Epoxy thoroughly using the tip of a pin or a lead of a resistor on a Petri dish.

2. Place the device on a clean surface, and carefully place masking tape to cover all holes except the electrode port.

3. Apply a small amount of the mixture inside the electrode port to fill-up the bottom half of the hole. Apply gentle dubbing pressure to remove any trapped air inside the port.

4. Insert the external electrode to be connected vertically up inside the electrode port, and hold it.

5. Apply more Silver Epoxy mixture at the bottom of the electrode. Eliminate any air bubble trapped in the hole by gently dubbing the mixture inside the port.

6. Repeat last two steps for all other electrodes to be connected.

7. Leave the device for about 5 minutes to allow the epoxy mixture to settle. Then use an ohm-meter to carefully verify connectivity by connecting one probe to the external electrode while the other probe connecting a flexible thin wire that is inserted into the cell port. If no connectivity, reapply the epoxy mixture. Repeat the procedure until proper connectivity is achieved.

8. Remove masking tape, and store the device with the epoxy in a secured place for about 24 hours to cure the epoxy.

9. After the epoxy is cured, verify that the electrodes were properly connected.

10. The last step is to solder wires with the external electrodes for connecting them with the external circuitry.

A photograph is given in Figure B.2 where the proper fixing of electrodes is being verified by using an ohm-meter. 


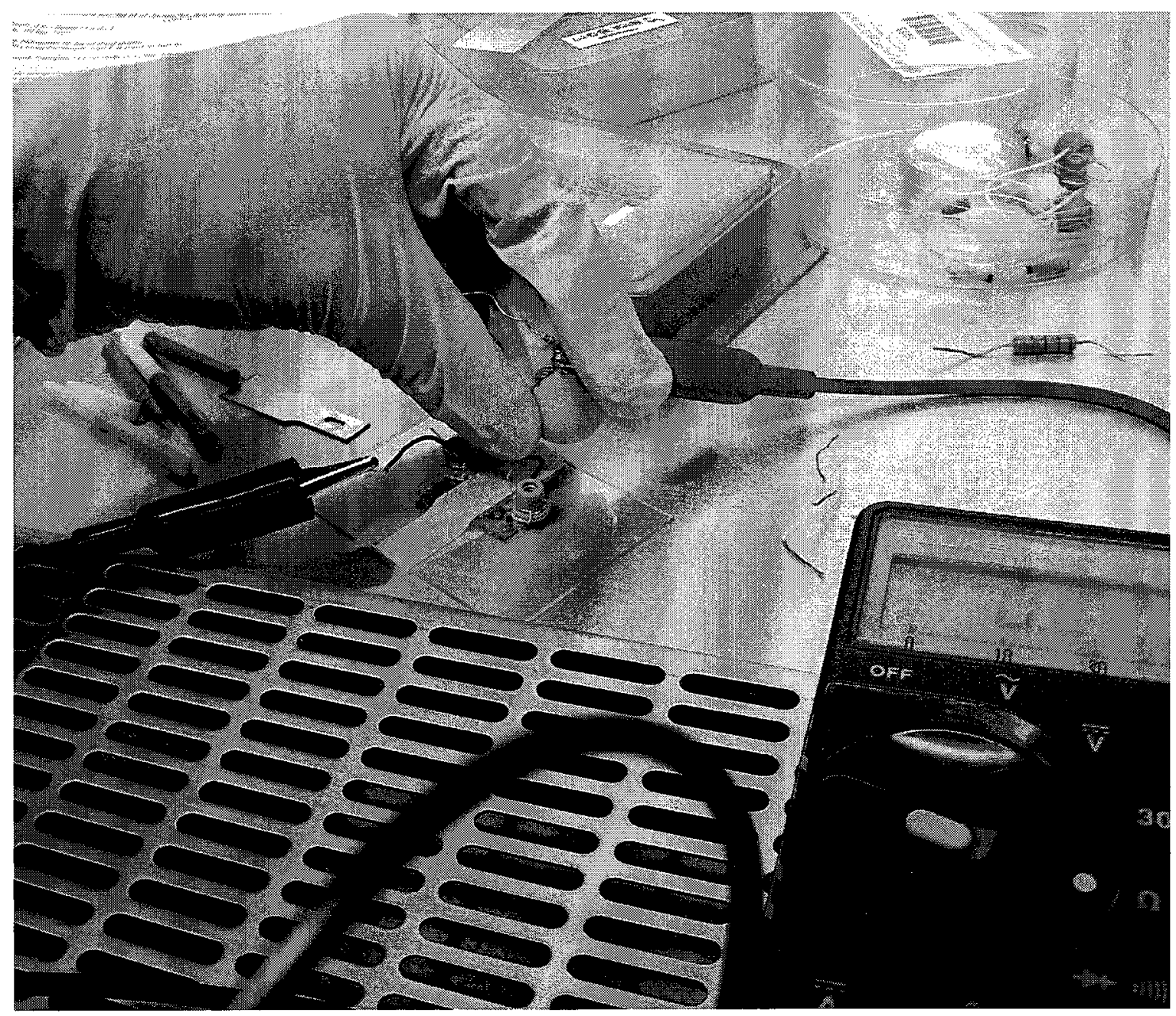

Figure B.2 A photograph showing a test to verify proper connectivity of an electrode with the integrated electrodes after silver epoxy bonding. 


\section{B.5 Developed protocol for reconnecting electrodes}

If after curing, it is tested that the external electrode became open-circuit with the internal electrode (eg. due to trapped air bubble), follow the procedure to remove cured silver epoxy and to re-apply.

1. Put masking tape on other holes beside the electrode hole of concern.

2. Place the device on top of a glass slide and place the setup on to of air holes in a lab work station with fans with suction air pressure (this will ensure the debris, mainly Silver epoxy, from the procedure will be sucked out of the device and surrounding area).

3. Use blade tip (of tube cutter, Upchurch scientific) to scrape off as much cured silver epoxy as possible.

4. Now use a fine set of spatula (Fisher scientific) in order of their sizes (from largest to smallest) to remove silver epoxy from the electrode hole (use circular motion).

5. Continue until internal (in-channel) electrode can be reached (verify using a multimeter and set of fine wires).

6. Wipe out any silver epoxy around the hole.

7. Reapply silver epoxy carefully ensuring proper connectivity and cure it (follow previous protocol).

The photograph given in Figure B.3 shows an electrode port is being removed for reconnection due to improper fixing in previous attempt.

\section{B.6 Developed protocol for fixing the device on a clean glass surface}

1. Place the device on top of the glass surface (eg. glass slide).

2. With tapes, secure top and bottom of the device to a suitable location, so that the device is tightly attached to the glass surface.

3. On both sides of the device (specially around the leak of fluid ports), deposit silicon gel and use a spatula to press the silicon so that there is no air bubble between silicon and the glass slide or the device.

4. After applying of silicon gel, let the setup dry for about 12 hours in room temperature 5. Remove tapes from top and bottom and apply silicon on top and bottom as before. 


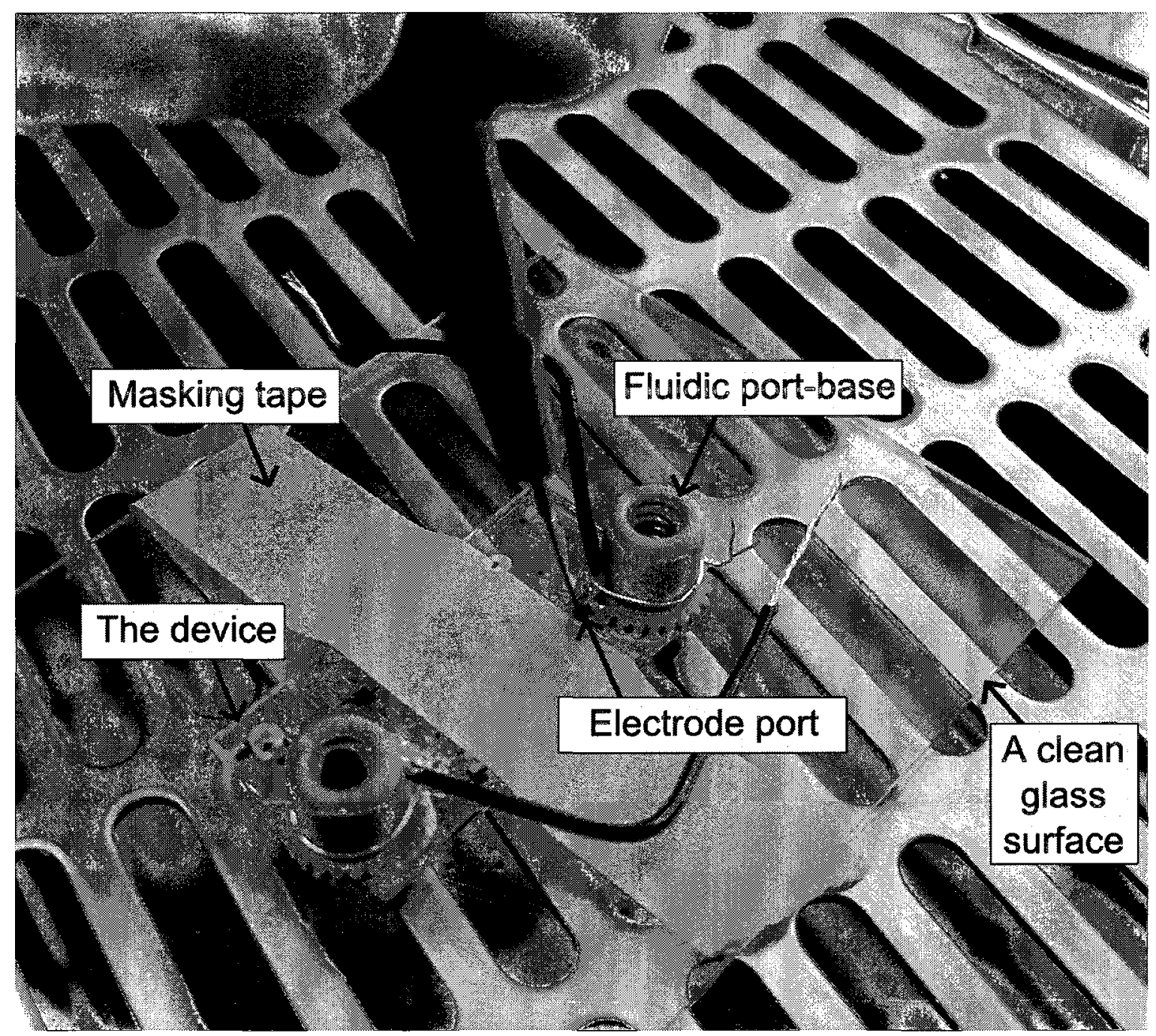

Figure $\mathbb{B}$.3 A photograph showing procedure for reconnecting the electrode on a sample device. The post-processed device has fluidic nano-port bases fixed. The masking tape covers all cell loading ports to prevent contamination and will be removed after post-processing is done. 
5. Let the setup dry out for another 24 hours in room temperature.

\section{B.7 Developed protocol for conditioning (wetting) of a microfluidic device}

This step is required once before the first use of a device.

Reagents required:

1. $1: 1(\mathrm{v} / \mathrm{v})$ detergent : D-PBS solution

2. $\mathrm{D}-\mathrm{H}_{2} \mathrm{O}$.

Protocol:

1. Add $30 \mu \mathrm{L}$ of the solution in both reservoirs of the device for at least 10 mins.

2. Use a micropipette to collect the solution through cell sample ports and reapply through fluidic ports.

3. Wash the device with flowing $\mathrm{D}-\mathrm{H}_{2} \mathrm{O}$ for at least 1 min and dry using blowing hot air for about 2 mins and then leave to dry in a moisture absorbing chamber (eg. dead air box).

4. Repeat the previous 3 steps for at least once more.

5. Add $5 \mu \mathrm{L}$ of $\mathrm{D}-\mathrm{H}_{2} \mathrm{O}$ to one of the fluidic port and observe the flow of the fluid. If the fluid flows through the channels to the fluidic port on the other side, finish the protocol, otherwise repeat the whole procedure.

\section{B.8 Developed protocol to repair a short circuited integrated electrode}

This protocol can be applied if there is a short circuit confirmed by an ohmic path between two electric ports (two electrodes) when the device is completely DRY. Typical value of a short circuited path is about $60 \Omega$, whereas open circuited ports should have resistances over $1 \mathrm{M} \Omega$ ). Attempt with a flow of $\mathrm{D}-\mathrm{H}_{2} \mathrm{O}$ through the ports (fluid and cell sample) of the device and let the device dry, then determine if the shortcircuit still exists. If short-circuit exists, it might be eliminated by applying a high current flow to induce joule heating using following protocol.

1. After the device is DRY, connect two wires from electric port to a DC power supply, with series current monitor.

2. Increase voltage across the electrodes and observe current flow. 
3. At the beginning, the current flow should be equal to the voltage divided by the resistance of that path.

4. Increase the magnitude of the applied voltage (about 12 to $15 \mathrm{~V}$ ).

5. At this level of excitation, there should be a sudden decrease of current flow (from $0.1 \mathrm{~A}$ to $1 \mathrm{~mA}$ or less, for example).

6. Stop increasing voltage at this point.

7. Connect to an ohm-meter to determine the resulted resistance. The resistance should show open-circuit as the short circuit is eliminated. 


\section{Appendix $\mathrm{C}$}

\section{Software Codes}

\section{C.1 Script to automate modelling, simulation and data extraction of MFD}

' Script For Maxwell 3D

' By Bashir Morshed (March 2007)

,

Option Explicit

Dim oAnsoftApp

Dim oDesktop

Dim oProject

Dim oDesign

Dim oEditor

Dim oModule

'Declaration for all loops

Dim ChLrange, ChWrange, ChHrange, OriLrange, OriWrange

ChLrange $=\operatorname{Array}(100,500,1000,5000,10000)$

ChWrange $=\operatorname{Array}(10,20,50,100,200,500)$

ChHrange $=\operatorname{Array}(10,20,50,100)$

'No orifice (narrow section)

OriLrange $=\operatorname{Array}(1)$

OriWrange $=\operatorname{Array}(1)$

'For loop begins

Dim a, b, c, d, e

For $\mathrm{a}=0$ To 4 'Ch Length

For $\mathrm{b}=0$ To 5 'Ch Widths

For $\mathrm{c}=0$ To 3 'Ch Heights

For $\mathrm{d}=0$ To 0 'Ori Lengths

For e $=0$ To 0 'Ori Widths 
'Declaration for each loop

Dim ChL, ChW, ChH, OriL, OriW

'All lengths here are in um

$\mathrm{ChL}=$ ChLrange(a)

$\mathrm{ChW}=$ ChWrange $(\mathrm{b})$

$\mathrm{ChH}=$ ChHrange(c)

OriL $=$ OriLrange $(\mathrm{d})$

OriW = OriWrange(e)

'Confirming OriW and OriL is smaller than ChW and ChL respectively

'(dimension check) turned OFF for fixed width case

'If (OriW i ChW) And (OriL ; ChL) Then

'Initializing project file

Dim DirName DirName = "homebmorshedResearchDesignsMaxwell3DChModelOneCh_extensive Dim FileName

FileName = "OneCh" \& CStr(ChL) \& "x" \& CStr(ChW) \& "x" \& CStr(ChH)

'Initializing

Set oAnsoftApp = CreateObject("AnsoftMaxwell.MaxwellScriptInterface")

Set oDesktop = oAnsoftApp.GetAppDesktop ()

oDesktop.RestoreWindow oDesktop.EnableAutoSave(false)

Set oProject $=$ oDesktop. NewProject

'Name of the Project oProject.Rename

DirName \& FileName \& ".mxwl", true

'Name of the Design

oProject.InsertDesign "Maxwell", FileName, "ElectroDCConduction", "“"

Set oDesign $=$ oProject.SetActiveDesign(FileName)

'Initialize Editor

Set oEditor = oDesign.SetActiveEditor("3D Modeler")

'Drawing of object begins

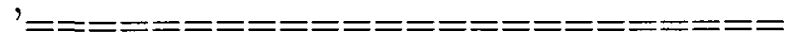

'Glass Plate

oEditor.CreateBox Array("NAME:BoxParameters", "CoordinateSystemID:=", -1, "XPosition:=", "Oum", "YPosition:=", "0um", "ZPosition:=", "Oum", "XSize:=", CStr(ChL+20+40) \& "um", "YSize:=", CStr $(\mathrm{ChW}+40) \&$ "um", "ZSize:=", CStr(ChH+40) \& "um"), -

Array("NAME:Attributes", "Name:=", "GlassPlate", "Flags:=", "", "Color:=", "(101 101 201)", "Transparency:=", 0.9, "PartCoordinateSystem:=", "Global", "MaterialName:=", "glass_PTFEreinf", "SolveInside:=", true)

'Channel

oEditor.CreateBox Array("NAME:BoxParameters", "CoordinateSystemID:=", -1, "XPosition:=", "30um", "YPosition:=", "20um", "ZPosition:=", "20um", "XSize:=", CStr(ChL) \& "um", "YSize:=",_

CStr(ChW) \& "um", "ZSize:=", CStr(ChH) \& "um"), 
Array ("NAME:Attributes", "Name:=", "Channel", "Flags:=", “"”, "Color:=", "(169 169 169)", "Transparency:=", 0.7, "PartCoordinateSystem:=", "Global", "MaterialName:=", "water_distilled", "SolveInside:=", true)

'Positive Electrode oEditor.CreateBox Array ("NAME:BoxParameters", "CoordinateSystemID:=", -1, "XPosition:=", "20um", "YPosition:=", "20um", "ZPosition:=", "20um", "XSize:=",

"10um", "YSize:=", CStr(ChW) \& "um", "ZSize:=", CStr(ChH) \& "um"), _ Array ("NAME:Attributes", "Name:=", "PosElec", "Flags:=","”, "Color:=", "(201 201 101)", "Transparency:=", 0.2, "PartCoordinateSystem:=", "Global", "MaterialName:=", "platinum", "SolveInside:=", true)

'Negative Electrode oEditor.DuplicateMirror Array("NAME:Selections", "Selections:=", "PosElec"), Array( "NAME:DuplicateToMirrorParameters", "DuplicateMirrorBaseX:=", CStr $((\mathrm{ChL} / 2)+10+20) \&$ "um", "DuplicateMirrorBaseY:=", $\operatorname{CStr}((\mathrm{ChW} / 2)+20)$ \& "um", "DuplicateMirrorBaseZ:=", CStr $((\mathrm{ChH} / 2)+20) \&$ "um", -

"DuplicateMirrorNormalX:=", "1um", "DuplicateMirrorNormalY:=", "0um", "DuplicateMirrorNormalZ:=", "Oum"), Array("NAME:Options",,

"DuplicateBoundaries:=", false) oEditor.ChangeProperty Array ("NAME:AllTabs",, Array("NAME:Geometry3DAttributeTab", Array("NAME:PropServers", "PosElec_1"), Array("NAME:ChangedProps", Array("NAME:Name", "Value:=", "NegElect"))))

'Channel and Electrode subtract from Glass plate oEditor.Subtract Array("NAME:Selections", "Blank Parts:=", "GlassPlate", "Tool Parts:=", "Channel,NegElect,PosElec"),

Array ("NAME:SubtractParameters", "CoordinateSystemID:=", -1 , "KeepOriginals: $=$ ", true)

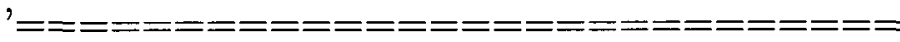

'Drawing of the object finished

'Saving only the mxwl file of the project oProject.Save

'Pausing after drawing —_ if required for debugging 'oDesktop.PauseScript "Model has been drawn successfully."

'Setting Excitation

Set oModule = oDesign.GetModule("BoundarySetup")

'Posive voltage oModule.AssignVoltage Array("NAME:PosElect1V", "Voltage:=", "1V", "CoordinateSystem:=", "", "Objects:=", Array ("PosElec"))

'Negative voltage oModule.AssignVoltage Array("NAME:NegElect0V", "Voltage:=", "0V", "CoordinateSystem:=", "", "Objects:=", Array ("NegElect")) 
'Setup Analysis

'For debugging "MaximumPasses:=" set to 2 instead of 30

Set oModule = oDesign.GetModule("AnalysisSetup")

oModule.InsertSetup "Electrostatic", Array("NAME:Setup1",,

"MaximumPasses:=", 30, "MinimumPasses:=", -

2, "MinimumConvergedPasses:=", 1, "PercentRefinement:=", 30, "LinearResidual:=",

1E-05, "ConvergenceDispParamId:=", -1, "SolveFieldOnly:=", false, "PercentError:=",

1, "SolveMatrixAtLast:=", true, "UseOutputVariable:=", false, "PreAdaptMesh:=",

false, "SolverType:=", 0)

'Saving the file of the project just BEFORE analyzing it

oProject.Save

'Analyze all

oDesign.AnalyzeAll

'Field Reporter

Set oModule = oDesign.GetModule( "FieldsReporter")

oModule.CreateFieldPlot Array("NAME:Mag_E1", "SolutionName:=",

"Setup1 : LastAdaptive", "QuantityName:=", "Mag_E", "PlotFolder:=", "E", "UserSpecifyName:=", -

0, "UserSpecifyFolder:=", 0, "IntrinsicVar:=", "”, "PlotGeomInfo:=", $\operatorname{Array}(1$,

"Surface", "FacesList", 6, "35", "36", "37", "38", "39", "40"), "FilterBoxes:=", Ar$\operatorname{ray}(1$, "Channel"), -

Array( "NAME:PlotOnSurfaceSettings", "Filled:=",

false, "IsoValType:=", "Tone", "SmoothShade:=", true, "AddGrid:=", false, "MapTransparency:=", -

true, "Transparency:=", 0, Array("NAME:Arrow3DSpacingSettings", "ArrowUniform:=", -

true, "ArrowSpacing:=", 0, "MinArrowSpacing:=", 0, "MaxArrowSpacing:=", 0), "GridColor:=", Array ( -

$255,255,255))$ )

'Saving the file of the project just AFTER analyzing it

oProject.Save

'Running Field Calculator

Set oModule $=$ oDesign.GetModule("FieldsReporter")

'Finding Maximum E-field

oModule.EnterQty "E"

oModule.CalcOp "Mag"

oModule.EnterVol "Channel"

oModule.CalcOp "Maximum"

oModule.ClcEval "Setup1 : LastAdaptive", Array()

'Saving Maximum E-field 
oModule.CalculatorWrite -

DirName \& "PersonalLib/Data/" \& FileName \& "_Max.txt", Array("Solution:=", _ "Setup1 : LastAdaptive"), Array()

'Finding Minimum E-field

oModule.EnterQty "E"

oModule.CalcOp "Mag"

oModule.EnterVol "Channel"

oModule.CalcOp "Minimum"

oModule.ClcEval "Setup1 : LastAdaptive", Array()

'Saving Minimum E-field

oModule.CalculatorWrite -

DirName \& "PersonalLib/Data/" \& FileName \& "_Min.txt", Array("Solution:=", _ "Setup1 : LastAdaptive"), Array()

'Finding Amplification Factor

oModule.CalcStack "exch"

oModule.CalcStack "rlup"

oModule.CalcOp "'”

'Saving Amplification Factor

oModule.CalculatorWrite -

DirName \& "PersonalLib/Data/" \& FileName \& "Factor.txt", Array("Solution:=",

"Setup1 : LastAdaptive"), Array()

'Saving the project — if required

oProject.Save

'Pausing after analysis —— if required 'oDesktop.PauseScript "Model has been analyzed successfully."

'Closing the project

oDesktop.CloseProject FileName

'End statement for "If" (dimension check)

'Else

'End If

'For loop repeats

Next

Next

Next

Next

Next

'Quit Application

'oDesktop.QuitApplication 


\section{C.2 Verilog program written for pulse generation of the FPGA board}

Listing of controller module (top module):

module ControllerV5 (CLOCK_50, SW, KEY, LEDR, GPIO_0);

input CLOCK_50; // System Clock (50 MHz)

input [17:0] SW; // SPDT switches with following settings:

// SW[17]: Mode selection (0- Run, 1-Prog)

// SW[16:15]: Prog Setting (00- Ampl, 01- ON time, 10- OFF time, 11- Number of pulses)

// SW[6:3]: Set Magnitude (range: $0-15$ for Ampl case, 1-9 for rest of the cases)

// SW[2:0]: Set Exponent (range: 0-7), ignored for Ampl case

\begin{tabular}{|c|c|c|c|}
\hline input & {$[3: 0]$} & KEY; & $\begin{array}{l}/ / \text { Push switches (default }=\text { on) } \\
/ / \text { KEY }[0]: \text { Reset, KEY [3]: Start }\end{array}$ \\
\hline output & {$[17: 0]$} & LEDR; & $\begin{array}{l}\text { // Red LEDs output [35:0] GPIO_0; } \\
/ / \text { Dutput port } \\
/ / \text { 3-0: Ampl[3:0] binary } \\
/ / \text { : Pulse out, 5: NOT Pulse out } \\
/ / \text { 6: Pulses being outputted }\end{array}$ \\
\hline
\end{tabular}

reg $[17: 0] \quad$ LEDR;

reg [35:0] GPIO_0;

reg [3:0] Ampl; // DAC amplitude

reg [3:0] PONMag; // ON time in microsecond (Magnitude

portion)

reg [2:0] PONExp; // ON time in microsecond (Exponent portion)

reg [3:0] POFFMag; // OFF time in microsecond (Magnitude portion)

reg [2:0] POFFExp; // OFF time in microsecond (Exponent portion)

reg [3:0] PNumMag; // Number of pulses (Magnitude portion)

reg [2:0] PNumExp; // Number of pulses (Exponent portion)

reg [26:0] TickON; // ON time in microsecond (total)

reg [26:0] TickOFF; // OFF time in microsecond (total)

reg [26:0] PNumber; // Number of pulses (total)

reg StartPulses; // Isolate KEY[3] from start, Only in Run mode

wire [26:0] PON;

wire [26:0] POFF;

wire [26:0] PNum;

wire POut;// Wire connecting oPDut to GPIO_O[4]

wire NotPOut; // Wire connecting oNotPOut to GPIO_O [5]

wire PulseON; // Wire connecting oTotPulses to GPIO_O[6]

always $Q$ (posedge CLOCK_50) begin if $(\mathrm{KEY}[\mathrm{O}]==0) / /$ Reset key

begin LEDR [0] $<=0$; GPIO_O $<=0$; 


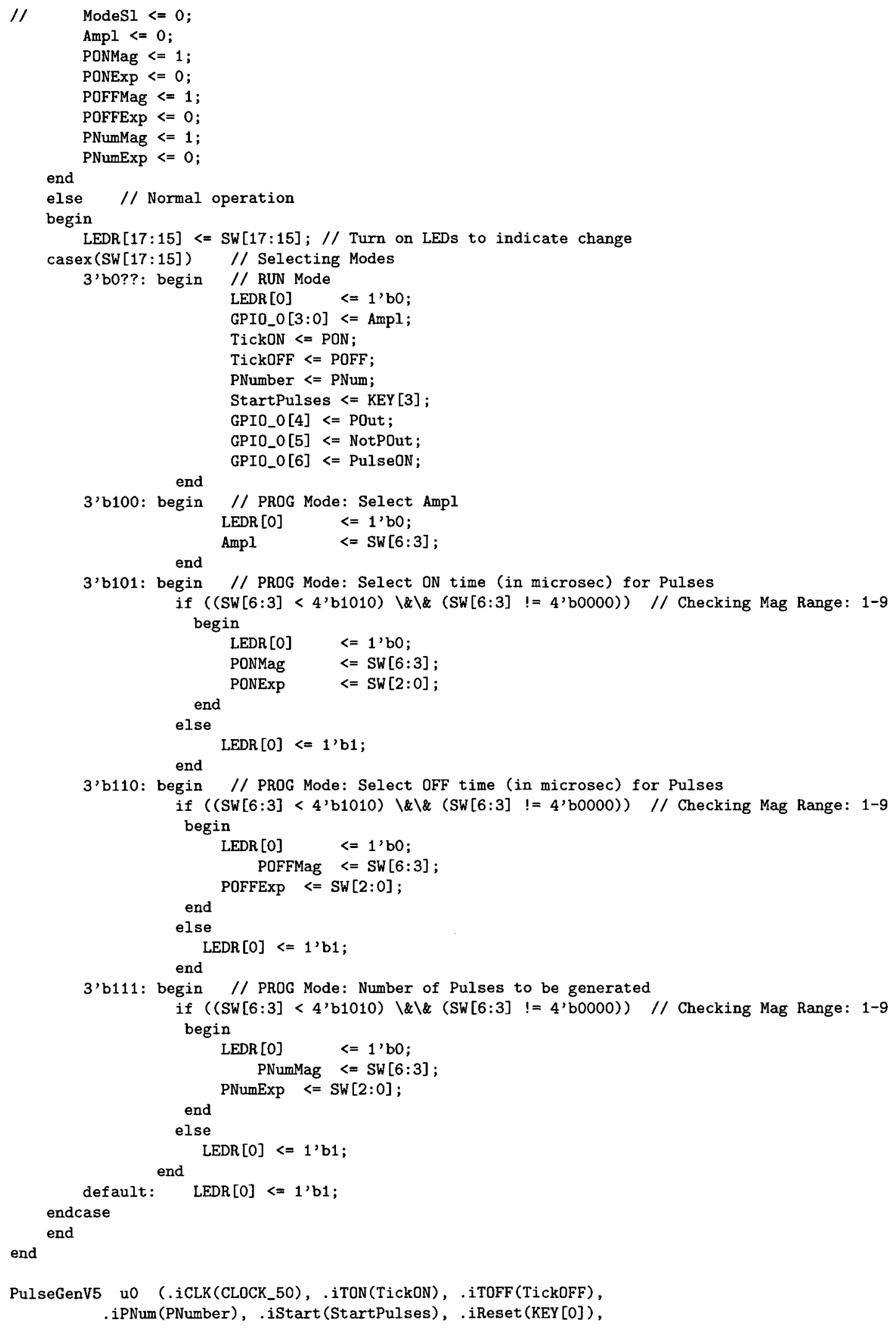


.oPOut (POut), .oNotPOut (NotPOut), .oPulseON(PulseON));

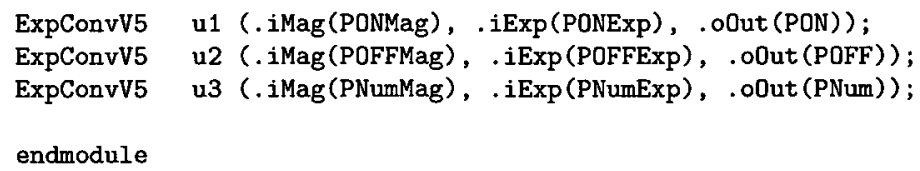

Listing for pulse generator module:

module PulseGenV5 (iCLK, iTON, iTOFF, iPNum, iStart, iReset, oPOut, oNotPOut, oPulse0N);

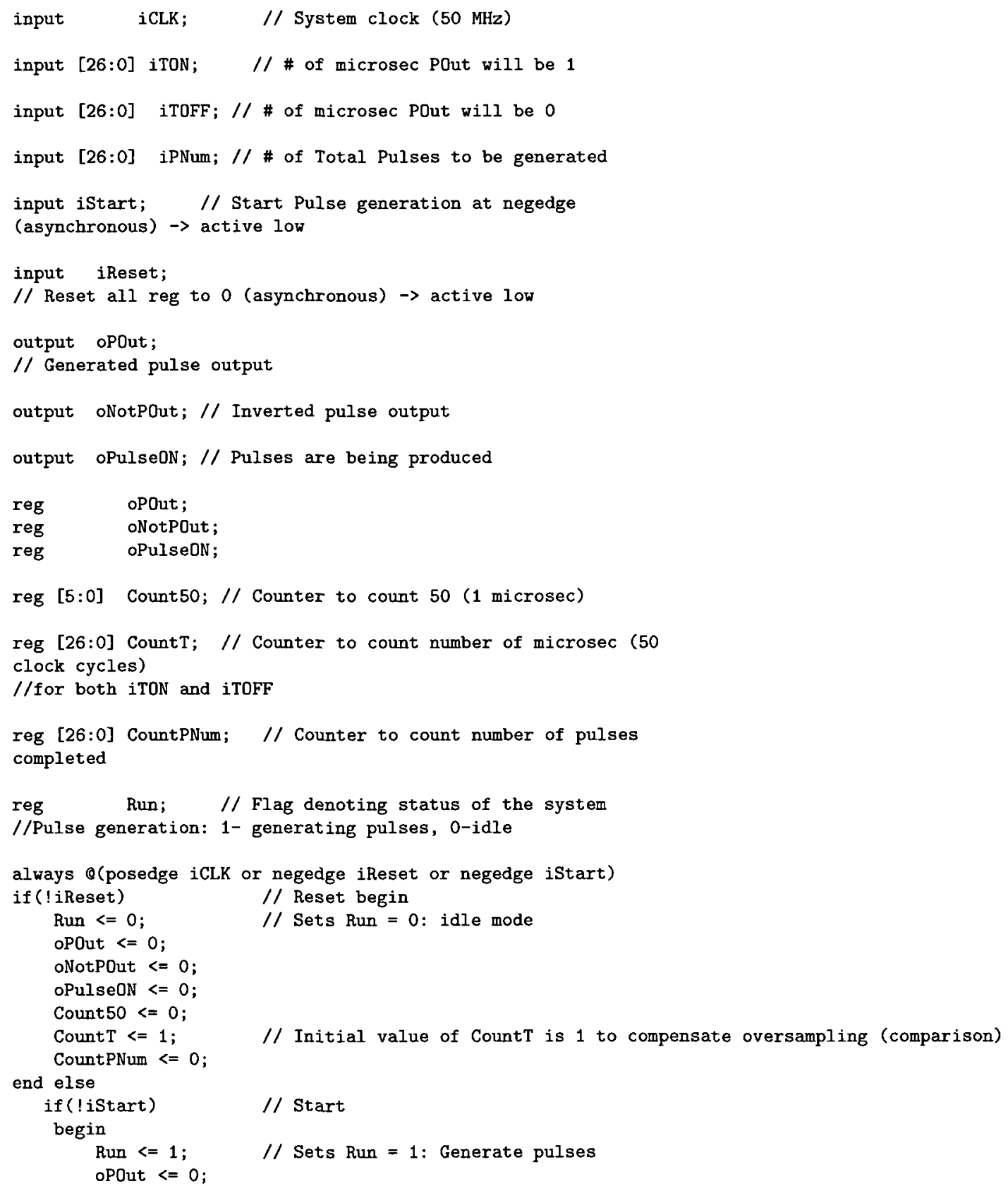




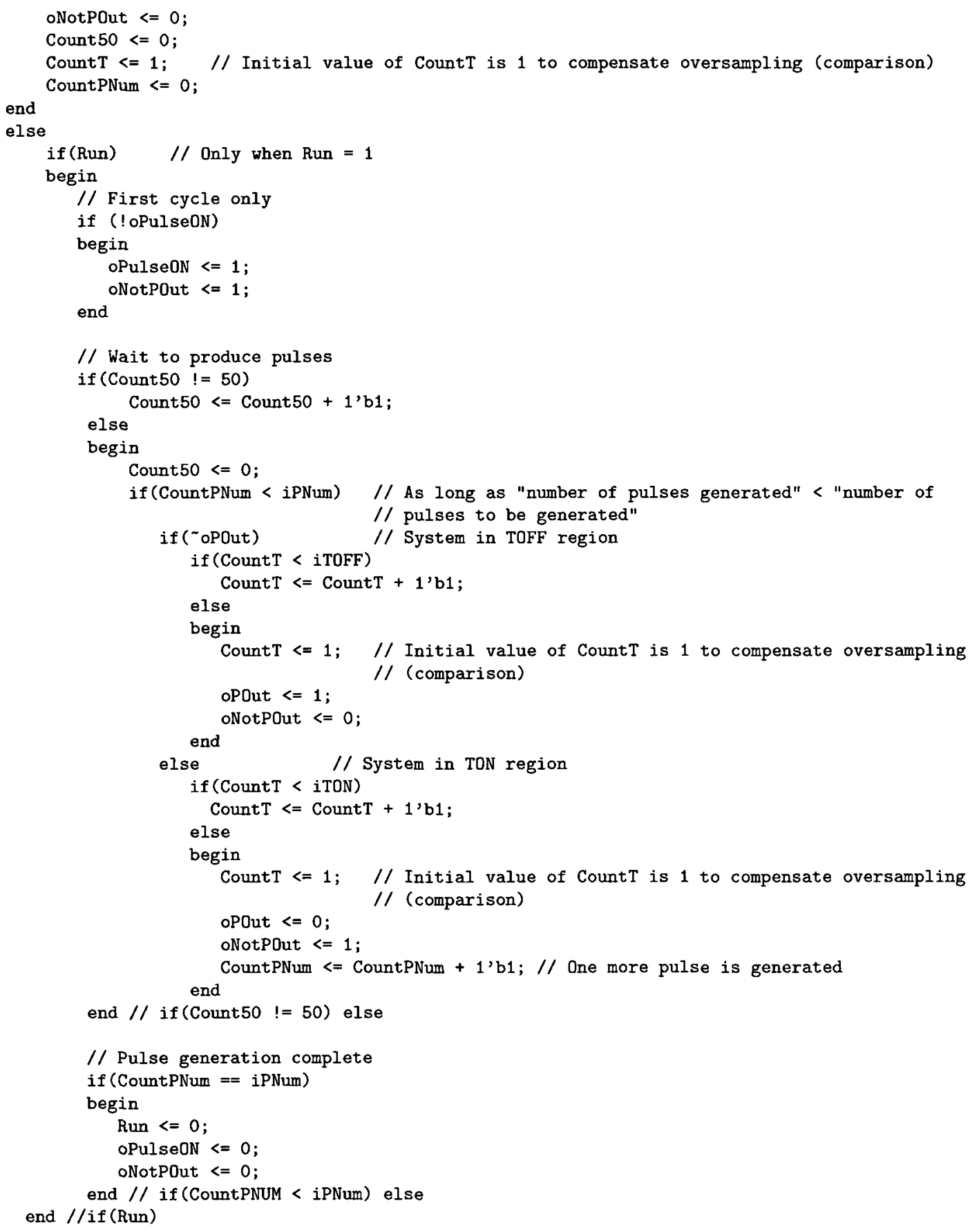

Listing of module for exponent converter:

module ExpConvV5 (iMag, iExp, oOut);

input [3:0] iMag; // Magnitude input

input [2:0] iExp; // Exponent input 


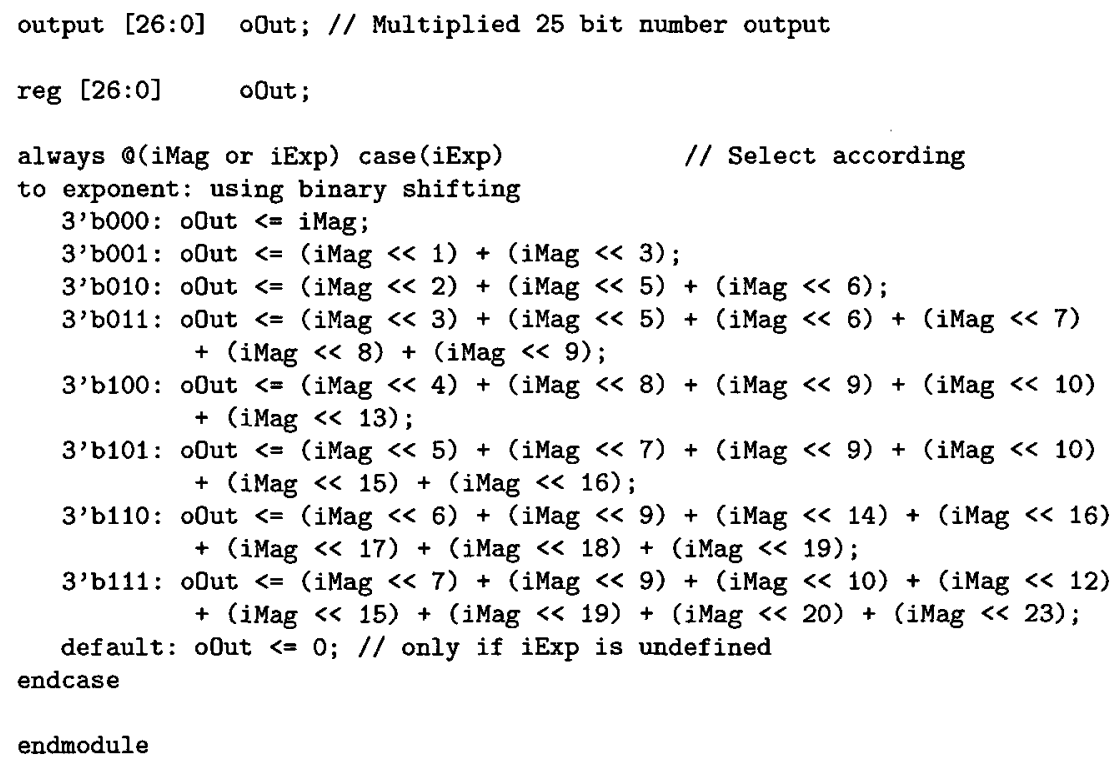

Listing of module for functional verification:

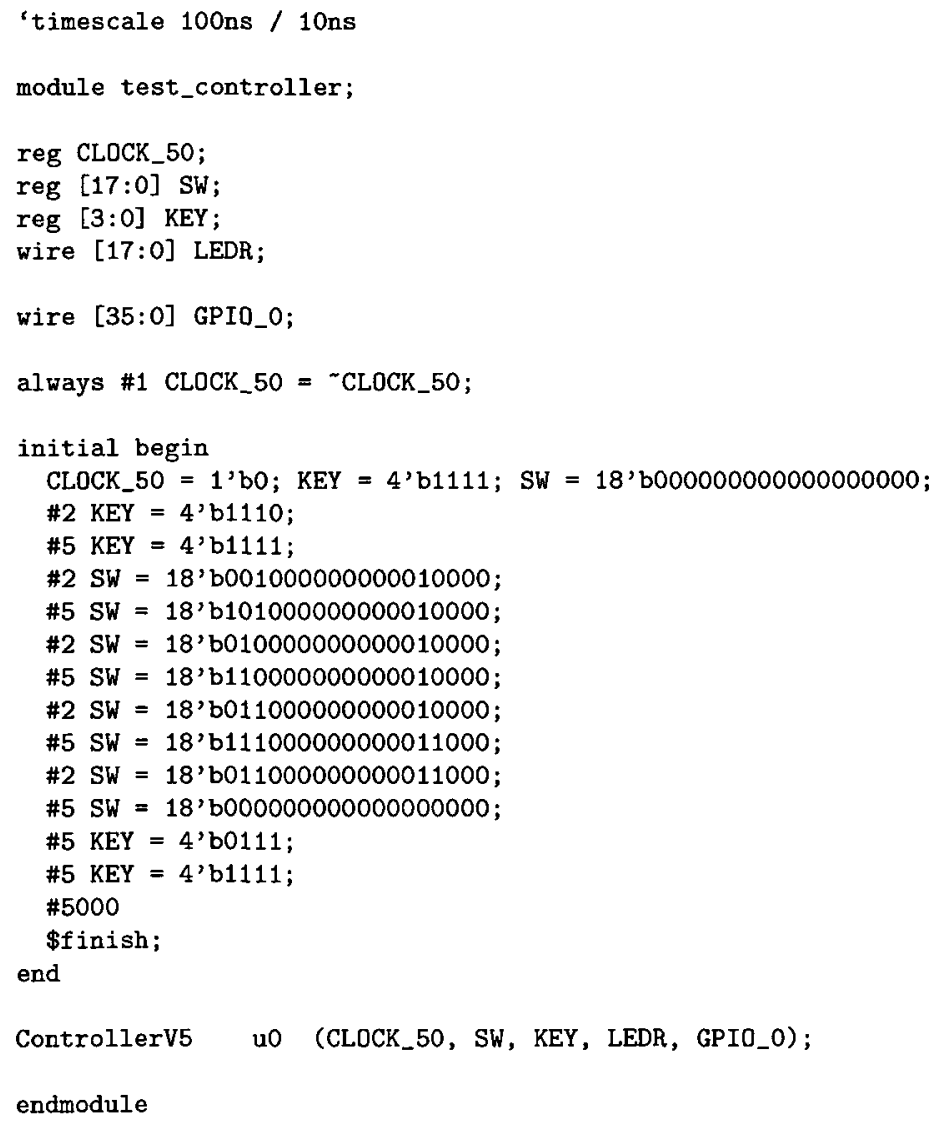

Some images of the FPGA board at various states of running conditions are shown below (Figure C.1 to C.5). 


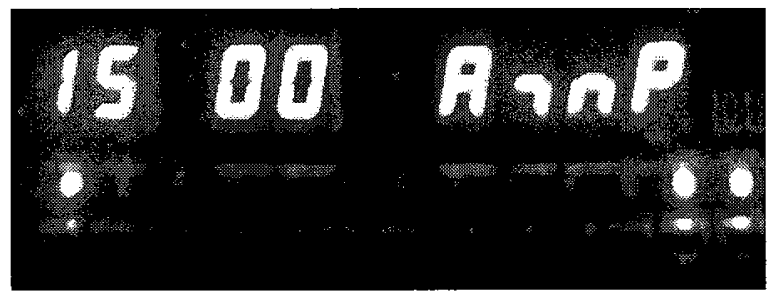

Figure C.1 The pulse amplitude is being set in the FPGA board.

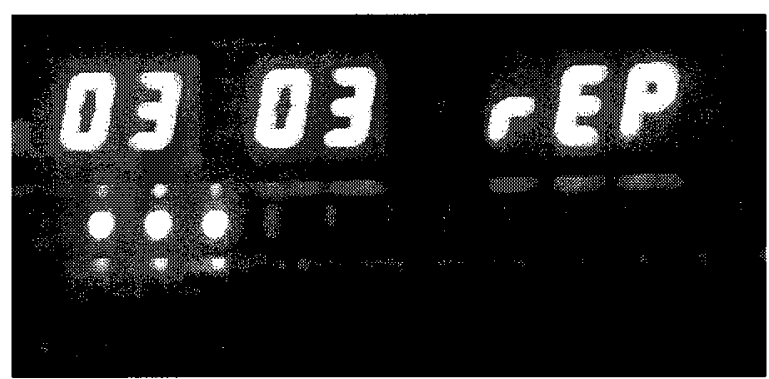

Figure C.2 The number of recitation of pulses is being set in the FPGA board.

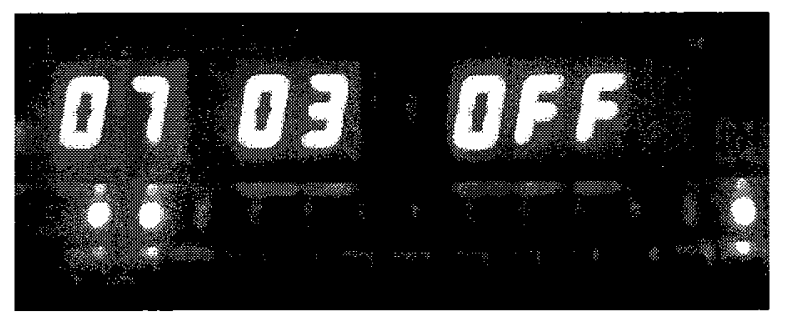

Figure C.3 Time duration for OFF period in between pulses (i.e. pulse gap) is being set in the FPGA board.

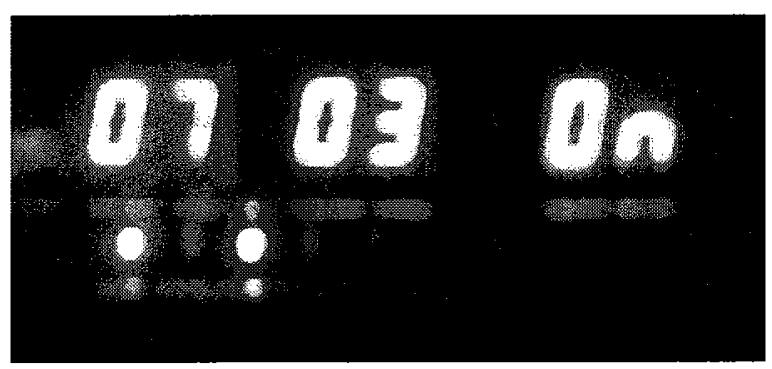

Figure C.4 Time duration for ON period (i.e. pulse width) is being set in the FPGA board. 


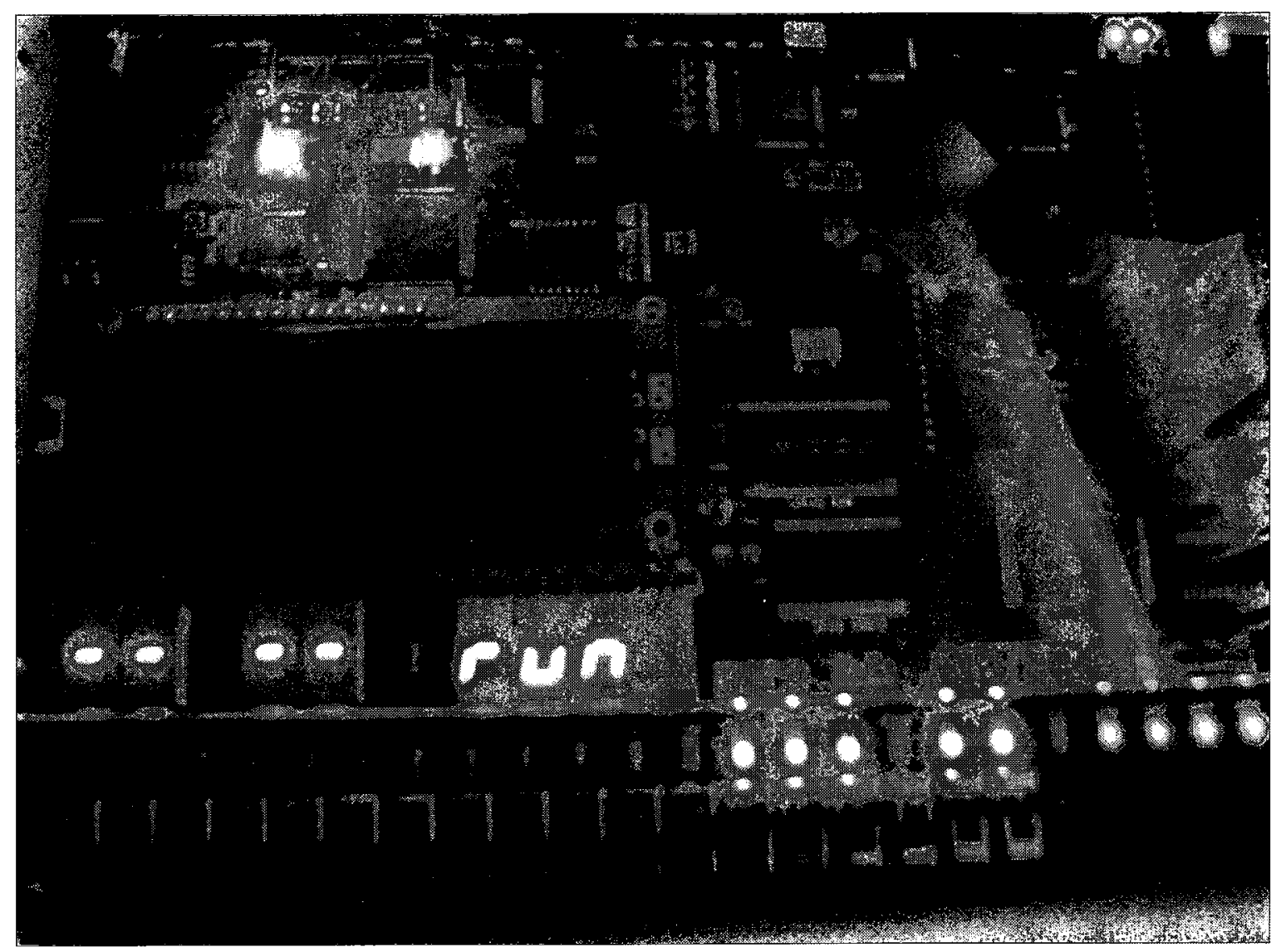

Tigure $\mathbb{C} .5$ The FPGA board in RUN state delivering pulses through the GPIO ports. 


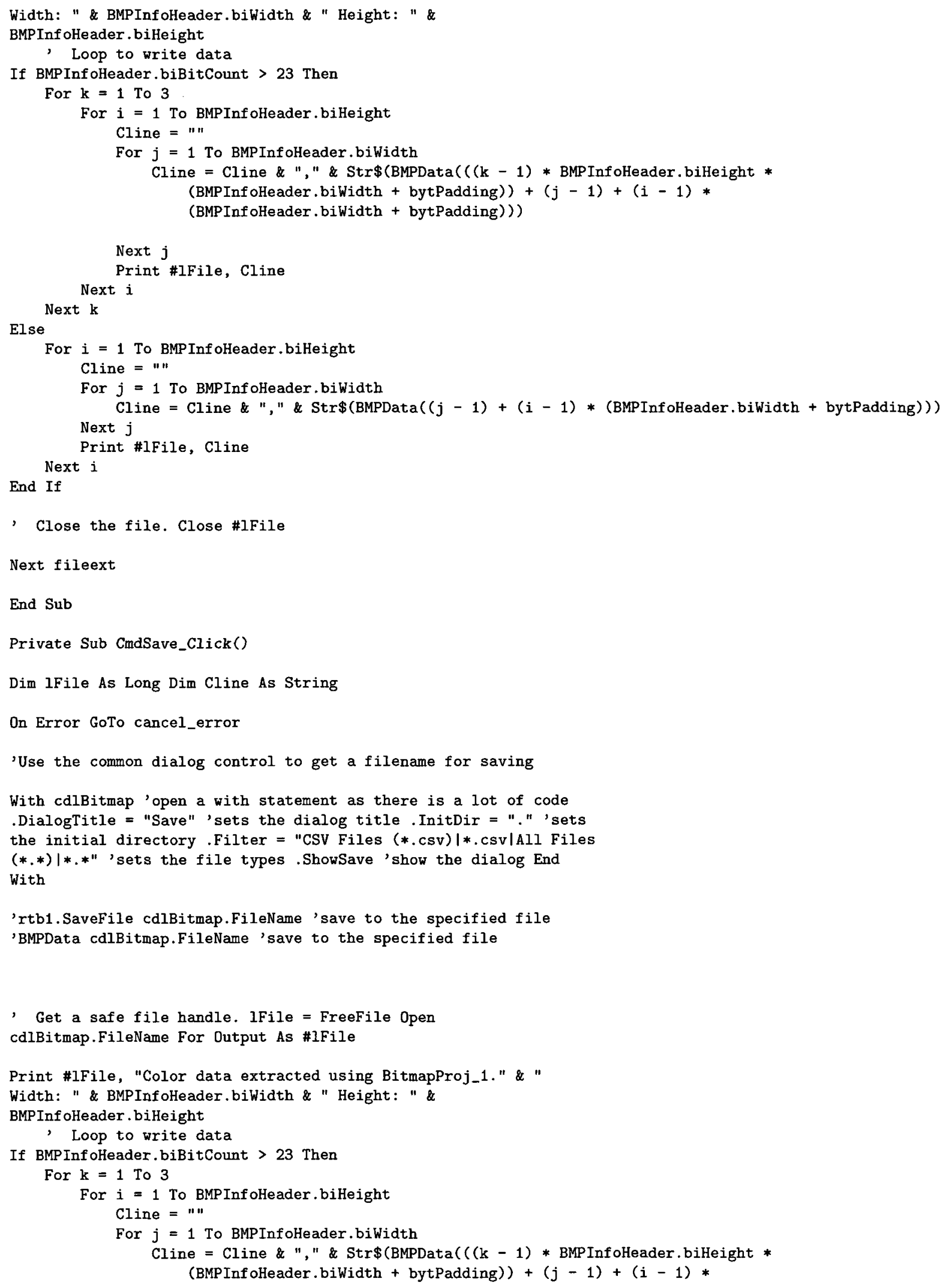


(BMPInfoHeader.biWidth + bytPadding)) )

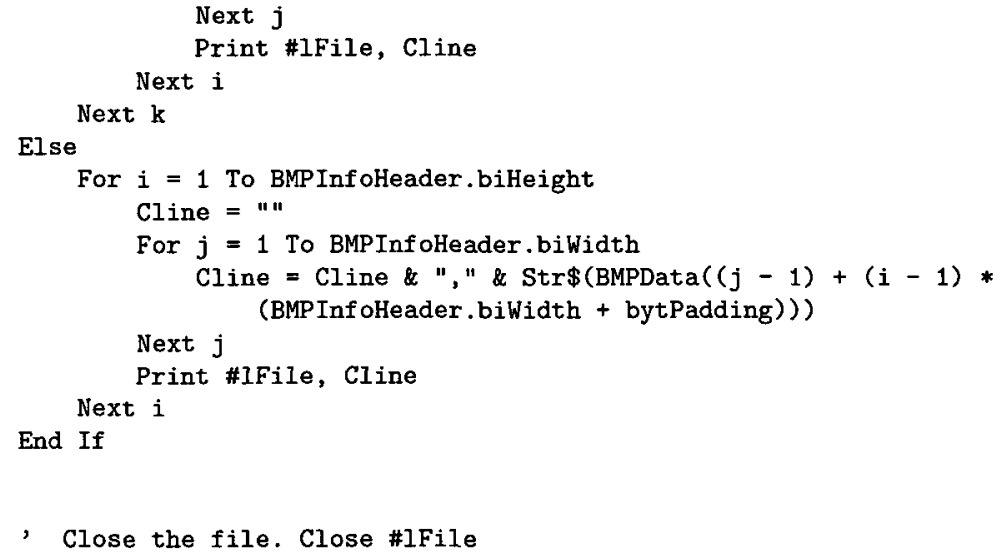

Exit Sub

cancel_error: If Err.Number $\longleftrightarrow$ cdlCancel Then MsgBox Err.Description End If

End Sub

Private Sub Form_Load()

'Load the sample bitmap

ExtractData App.Path \& "\sample.bmp", "sample.bmp"

End Sub

Private Sub cmaOpen_Click()

Const HideReadOnly $=\& H 4$ Const FileMustExist $=\& H 1000$

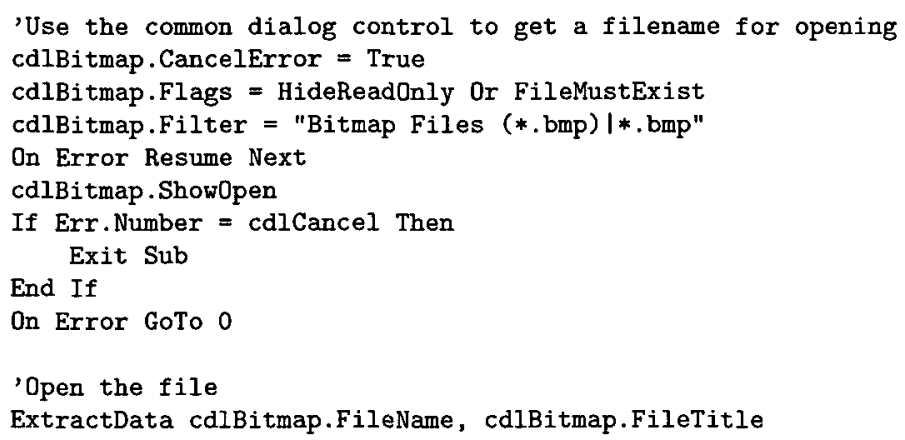


'Fill the File Header structure Get intBMPFile, 1, BMPFileHeader

'Fill the Info Header structure Get intBMPFile, , BMPInfoHeader

'How many bits per pixel are we dealing with?

Select Case BMPInfoHeader.biBitCount

Case 1

ReDim BMPRGBQuad(1)

Case 4

ReDim BMPRGBQuad (15)

Case 8

ReDim BMPRGBQuad (255)

End Select

'If it's less than 24bit, then get the colour data

If BMPInfoHeader.biBitCount $<24$ Then

'Fill the colour table

Get intBMPFile, , BMPRGBQuad()

End If

'Size the BMPData array

ReDim BMPData(BMPInfoHeader.biSizeImage)

'Fill the BMPData array

Get intBMPFile, , BMPData

close intBMPFile

'Display the File Header Data

With BMPFileHeader

TempString = "bfType: " \& BMPFileHeader.bfType

TempString $=$ TempString \& vbNewLine \& "bfSize: " \& .bfSize

TempString $=$ TempString \& vbNewLine \& "bfReserved1: " \& .bfReserved1

TempString $=$ TempString \& vbNewLine \& "bfReserved2: " \& .bfReserved2

TempString = TempString \& vbNewLine \& "bfOffBits: " \& .bfOffBits End With

'Output the File Header data

lblFileHeader . Caption $=$ TempString

Tempstring = " "

'Display the Info Header Data

With BMPInfoHeader

TempString = "biSize: " \& .biSize

TempString $=$ TempString \& vbNewLine \& "biWidth: " \& .biWidth

TempString $=$ TempString \& vbNewLine \& "biHeight: " \& .biHeight

TempString $=$ TempString \& vbNewLine \& "biPlanes: " \& .biPlanes

TempString $=$ TempString \& vbNewLine \& "biBitCount: " \& .biBitCount

TempString $=$ TempString \& vbNewLine \& "biCompression: " \& .biCompression

TempString $=$ TempString \& vbNewLine \& "biSizeImage: " \& .biSizeImage

TempString $=$ TempString \& vbNewLine \& "biXPelsPerMeter: "\& .biXPelsPerMeter

TempString $=$ TempString \& vbNewLine \& "biYPelsPerMeter: "\& .biYPelsPerMeter

TempString $=$ TempString \& vbNewLine \& "biClrUsed: " \& .biClrUsed

TempString $=$ TempString \& vbNewLine \& "biClrImportant: " \& .biClrImportant

End With

'Output the Info Header data

lblinfoHeader. Caption $=$ Tempstring

Tempstring $=" n$

'Set up the colour table scroll bar and picture

If BMPInfoHeader.biBitCount < 24 Then

scrTable. Enabled $=$ True

scrTable. Min $=0$

scrTable. Max = UBound (BMPRGBQuad)

scrTable_Change

Else

lblTable $=$ "Colour Table (NONE)"

scrTable. Enabled = False 
End If

'Find out how much padding there will be at the end of each data row (if any) bytPadding $=32-($ (BMPInfoHeader.biWidth * BMPInfoHeader. biBitCount) Mod 32) If bytPadding $=32$ Then bytPadding $=0$

bytPadding $=$ bytPadding $\backslash$ BMPInfoHeader, biBitCount

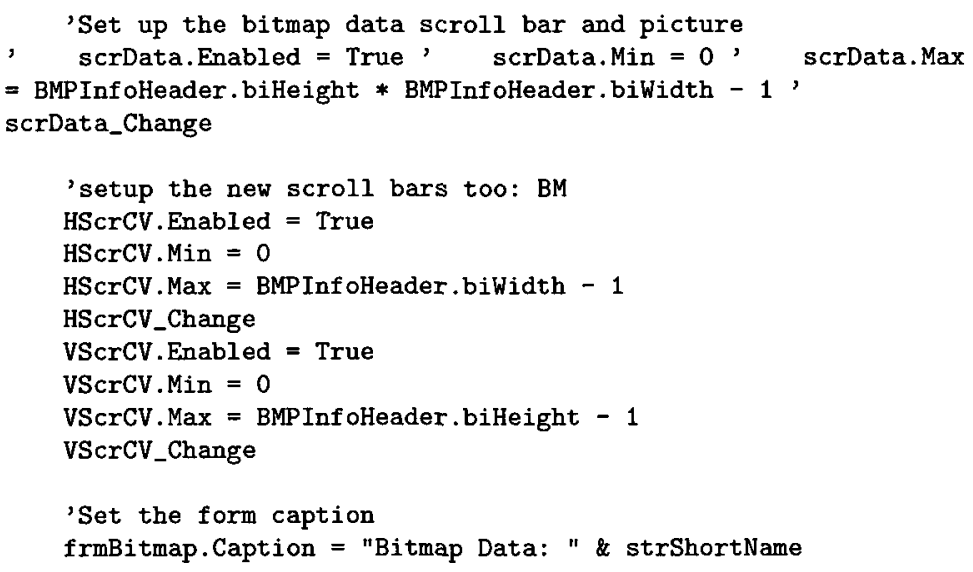

End Sub

Private Sub HScrCV_Change()

Dim tmpPixel As Long

'Change the picture colour and text appropriately

If BMPInfoHeader.biBitCount > 23 Then

lblCV.Caption = HScrCV.Value + $1 \&$ "X" \& VScrCV.Value + $1 \&$ ": " \& GetPixelColour (BMPInfoHeader.biBitCount, HScrCV.Value + VScrCV.Value * (BMPInfoHeader.biWidth + bytPadding), BMPData)

tmpPixel = HScrCV.Value + VScrCV.Value * (BMPInfoHeader.biWidth + bytPadding)

lblCV.Caption = lblCV. Caption \& " R " \& BMPData(tmpPixel * 3) \& " G " \& BMPData(tmpPixel *3+1) \& 3 " \& BMPData(tmpPixel *3+2)

IblCV.Caption = HScrCV.Value + 1 \& "X" \& VScrCV.Value + 1 \& ":" \& End If BMPData(HScrCV.Value + VScrCV.Value * (BMPInfoHeader.biWidth + bytPadding))

lblCV.BackColor = GetPixelColour (BMPInfoHeader.biBitCount, HScrCV.Value + VScrCV.Value * (BMPInfoHeader.biWidth + bytPadding), BMPData)

End Sub

Private Sub scrData_Change()

'Change the picture colour and text appropriately (account for possible padding!) lblData = "Bitmap Data (" scrData.Value \& ")"

picData.BackColor = GetPixelColour (BMPInfoHeader.biBitCount, scrData.Value + (scrData.Value \BMPInfoHeader.biWidth) * bytPadding, BMPData)

End Sub

Private Sub scrTable_Change()

'Change the picture colour and text appropriately lblTable = "Colour Table (" \& scrTable.Value \& ")"

picTable. BackColor = RGB (BMPRGBQuad (scrTable.Value).rgbRed,

BMPRGBQuad (scrTable.Value).rgbGreen, BMPRGBQuad (scrTable.Value).rgbBlue) 
Private Function GetPixelColour(intBPP As Integer, lngPixelnum As Long, bytData() As Byte) As Long

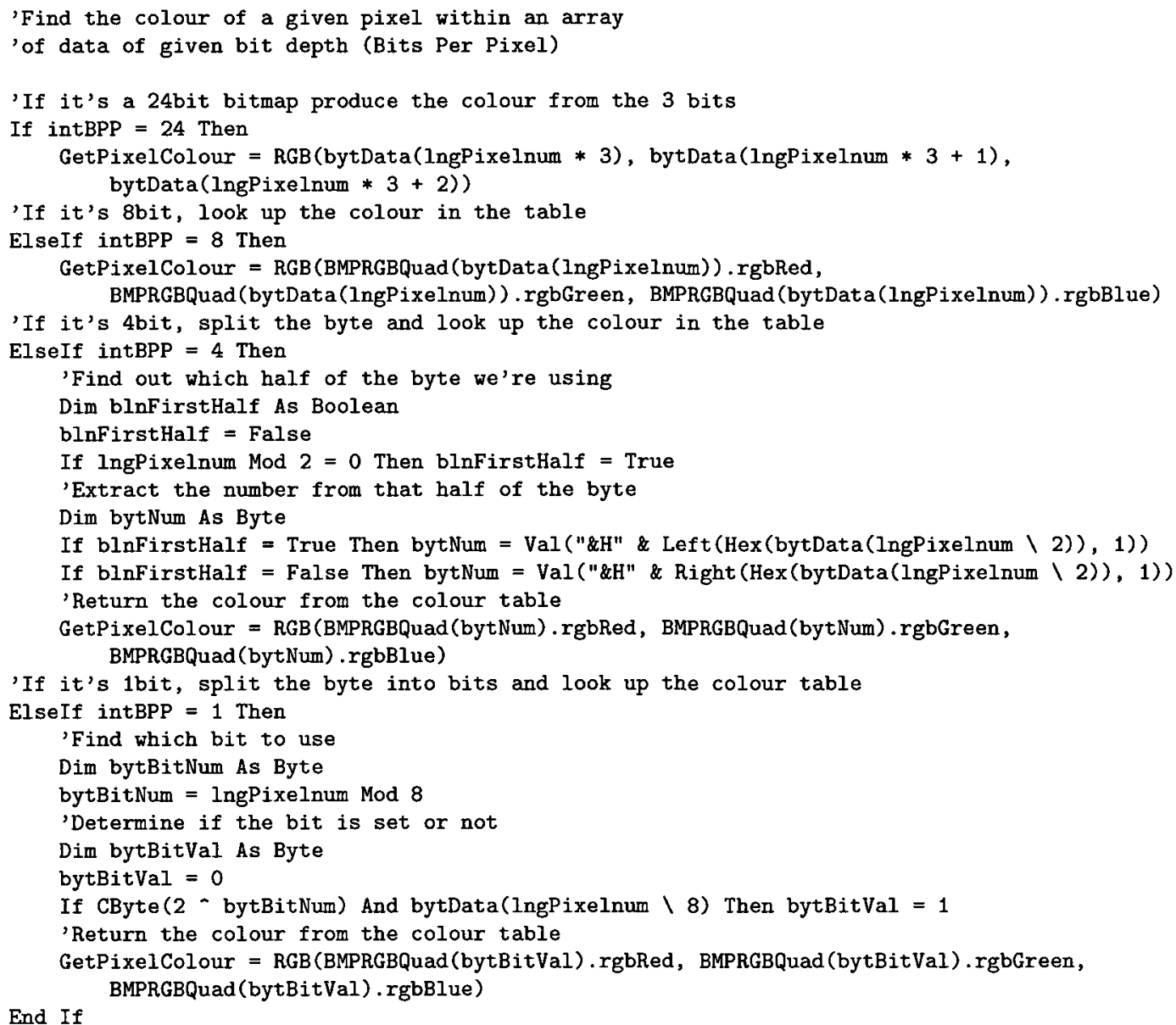

End Function

Private Sub VScrCV_Change()

Dim tmpPixel As Long

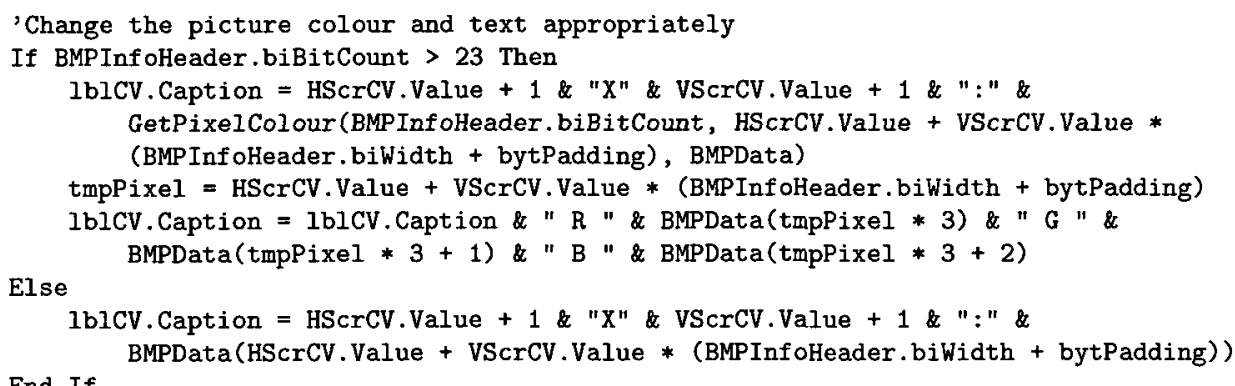
End If

lblCV.BackColor $=$ GetPixelColour (BMPInfoHeader.biBitCount, HScrCV.Value + VScrCV.Value * (BMPInf oHeader.biWidth + bytPadding), BMPData)

End Sub 


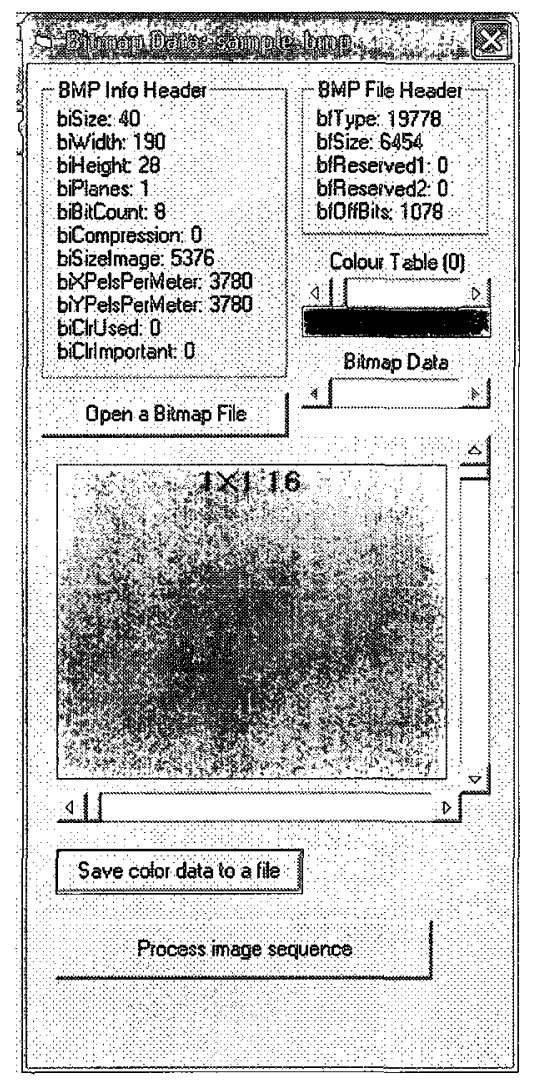

Figure $\mathbb{C} .6 \mathbb{A}$ snapshot of the visual basic (VB) program interface.

A snapshot of the program interface is given in Figure C.6.

\section{C.4 Matab code to analyze pizel data information and to create plots}

clear all;

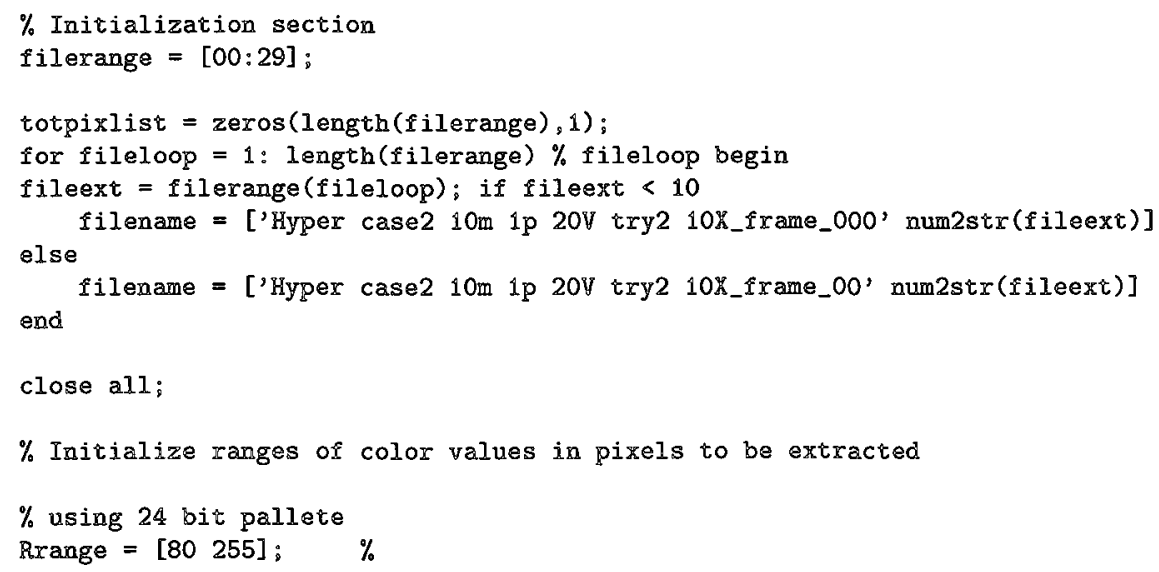




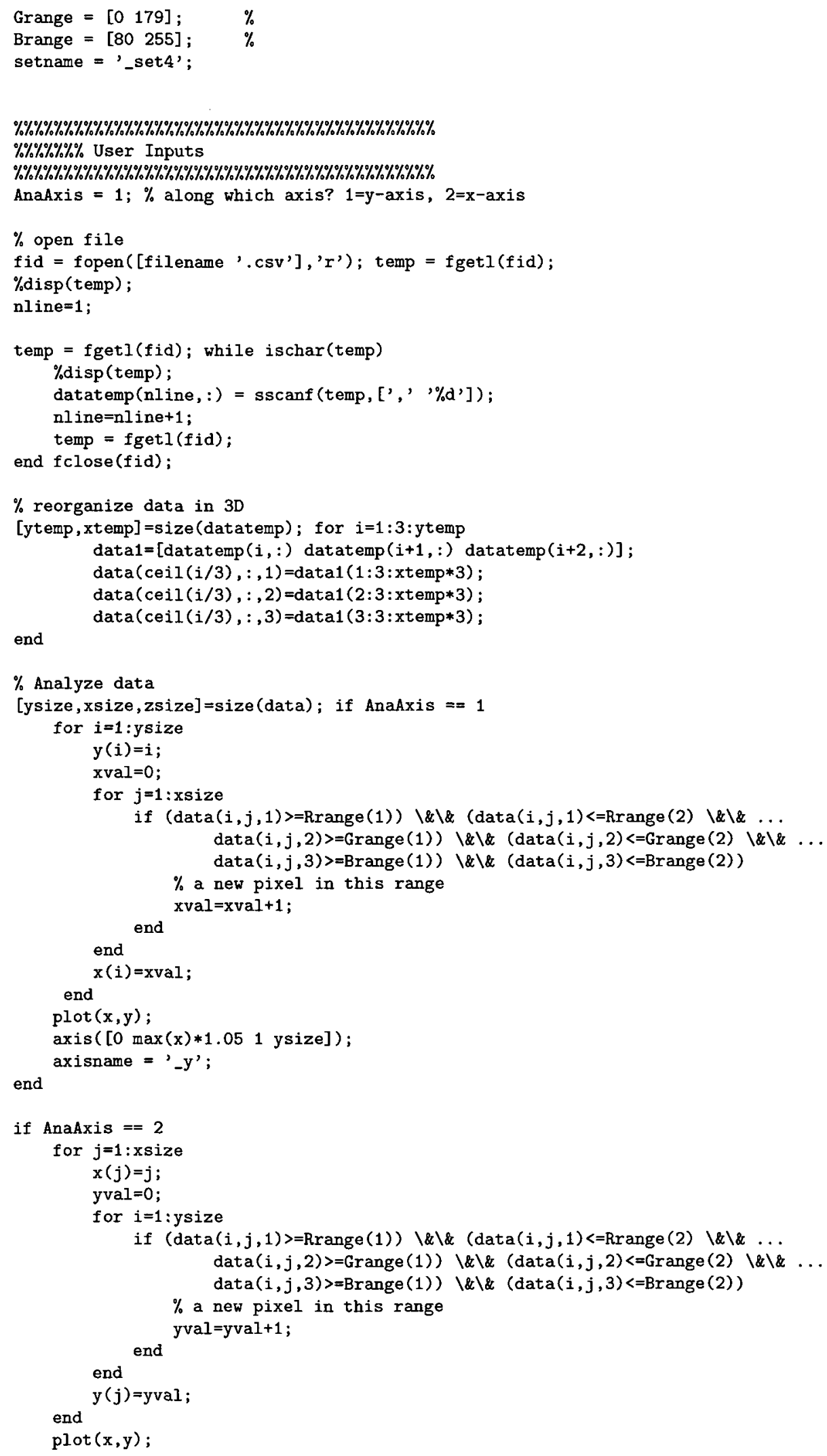




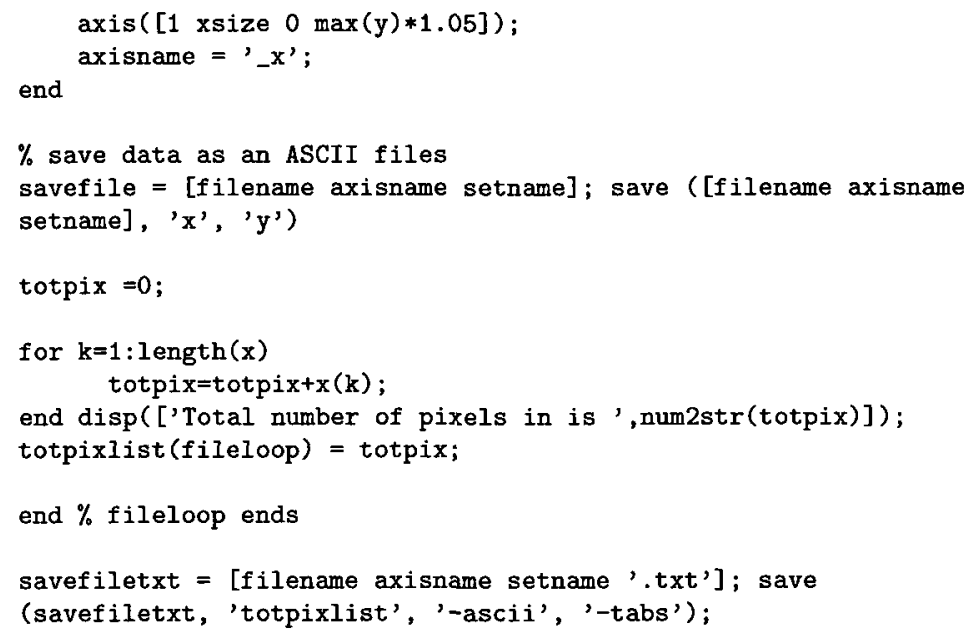

\section{C.5 Comsol mesh parameters}

With the predefined "extremely fine" mesh option, parameters were set as follows:

Maximum element size scaling factor: 0.2

Maximum growth rate: 1.3

Mesh curvature factor: 0.2

Mesh curvature cutoff: 0.001

Resolution of narrow region: 1

For thermal simulation, these parameters resulting a mesh with 2258159 tetrahedron elements, 385863 mesh points and 3045076 degrees of freedom. Number of triangular boundary elements were 196216 and number of edge elements were 11532 . Minimum element quality was 0.2258 with element volume ratio of $3.83 \times 10^{-8}$. 


\section{Bibliography}

[1] E. Neumann, A. E. Sowers, and C. A. Jordan, Electroporation and electrofusion in cell biology, Plenum Press, NY, USA, 1989.

[2] Q. Hu, V. Shidhara, R. P. Joshi, J. F. Kolb, and K. H. Schoenbach, "Molecular dynamics analysis of high electric pulse effects on bilayer membranes containing DPPC and DPPS," IEEE Trans. Plasma Science, vol. 34, no. 4, pp. 1405-1411, 2006.

[3] Q. Hu, S. Viswanadham, R. P. Joshi, K. H. Schoenbach, S. J. Beebe, and P. F. Blackmore, "Simulations of transient membrane behavior in cells subjected to a high-intensity ultrashort electric pulse," Physical Review, vol. 71, no. 3, pp. 031914-1-9, 2005.

[4] K. H. Schoenbach et al., "Ultrashort electrical pulses open a new gateway into biological cells," Proc. IEEE, vol. 92, no. 7, pp. 1122-1137, 2004.

[5] J. D. Woodward, N. M. Orlans, and P. t. Higgins, Biometrics, McGraw-Hill, CA, USA, 2003.

[6] P. Liu, S.H.I. Yeung, K. A. Crenshawc, C. A. Crouse, J. R. Scherer, and R. A. Mathies, "Real-time forensic DNA analysis at a crime scene using a portable microchip analyzer," Forensic Science Intl: Genetics, vol. 2, pp. 301-309, 2008.

[7] J. Gans and G. Urbas, "DNA identification in the criminal justice system," Trends and issues in crime and criminal justice, , no. 226, pp. 5 pages, 2002.

[8] R. Blundell, "DNA fingerprinting - review paper," Res. J. Applied Sci., vol. 1, no. 4, pp. 136-140, 2006.

[9] C. M. Ruitberg, D. J. Reeder, and J. M. Butler, "STRBase: a short tandem repeat DNA database for the human indentiy testing community," Nucleic Acids Research, vol. 29, no. 1, pp. 320-322, 2001.

[10] B. Alberts et al., Essential cell biology: an introduction to the molecular biology of the cell, Garland Publishing, NY, USA, 1997. 
[11] H. Lodish et al., Molecular cell biology, W. H. Freeman and Co., New York, 2003.

[12] P. N. Prasad, Introduction to biophotonics, Wiley Interscience, NJ, USA, 2003.

[13] J. Dwyar, P. Neufeld, and B. Scheck, Actual innocence: when justice goes wrong and how to make it right, New Americn Library, NY, USA, 2003.

[14] Federal Bureau of Investigation, "Combined DNA index system," Internet: www.fbi.gov/hq/lab/html/codis1.htm, Sept. 2007, [revised June 15, 2009].

[15] A. D. Baxevanis and B. F. F. Ouellette, Bioinformatics: a practical guide to the analysis of genes and proteins, Wiley-Interscience, NY, USA, 2001.

[16] Y. P. Bao, M. Huber, T. Wei, S. S. Marla, J. J. Storhoff, and U. R. Muller, "SNP identification in unamplified human genomic DNA with gold nanoparticle probes," Nucleic Acids Research, vol. 33, no. 3, pp. e15, 7 pages, 2005.

[17] E. Giardina, I. Pietrangeli, C. Martone, P. Asili, I. Predazzi, and P. Marsala et al., "In silico and in vitro comparitive analysis to select, validate and test SNPs for human identification," BMC Genomics, vol. 8, no. 457, pp. 7 pages, 2007.

[18] H. Chun et al., "Extraction of gene-disease relations from Medline using domain dictionaries and machine learning," Pacific Symposium on Biocomputing, vol. 11, pp. 4-15, 2006.

[19] GEN2PHEN Project, "GEN2PHEN knoledge centre," Internet: www.gen2phen.org/about, Jan. 2009, [June 15, 2009].

[20] S. C. Dixon, J. Horti, Y. Guo, E. Reed, and W. D. Figg, "Methods for extracting and amplifying genomic DNA isolated from frozen serum," Nature Biotechnology, vol. 16, pp. 91-94, 1998.

[21] J. Kieleczawa, DNA sequencing: optimizing the process and analysis, Jones and Bartlett Publishers, MA, USA, 2005.

[22] P. C. Turner, A. G. McLennan, A. D. Bates, and M. R. H. White, Molecular biology, Springer-Verlag, New York, USA, 2000.

[23] B. M. Paegel, C. A. Emrich, G. J. Wedemayer, J. R. Scherer, and R. A. Mathies, "High throughput DNA sequencing with a microfabricated 96-lane capillary array electrophoresis bioprocessor," in Proc. Nat. Academy Sci. USA, 2002, vol. 99, pp. 574-579.

[24] R. H. Liu et al., "Integrated microfluidic DNA array biochips," in Micro Total Analysis Systems, 2001, pp. 465-467. 
[25] S. Knudsen, A biologist's guide to analysis of DNA microarray data, Wiley-liss, NY, USA, 2002.

[26] G. M. Malacinski, Essentials of molecular biology, Jones and Bartlett Publishers, MA, USA, 2003.

[27] G. A. Urban, BioMEMS, Springer, Netherlands, 2006.

[28] S. S. Saliterman, Fundamentals of bioMEMS and medical microdevices, WileyInterscience, WA, USA, 2006.

[29] M. Viaznikova and T. Mussivand, "New technique for DNA extraction from non-invasive samples," in Bio-, micro-, and nanosystems, 2003, pp. 26-27.

[30] M. Schienle, A. Frey, F. Hofmann, B. Holzapfl, C. Paulus, P. Schindler-Bauer, and R. Thewes, "A fully electronic DNA sensor with 128 positions and in-pixel A/D conversion," in Int. Solid State Circuits Conf., 2004, p. S:12.2.

[31] M. Schienle et al., "A fully electronic DNA sensor with 128 positions and in-pixel A/D conversion," IEEE J. Solid-state Circuits, vol. 39, no. 12, pp. $2438-2445,2004$.

[32] A. Frey et al., "A digital CMOS DNA chip," in IEEE Intl Symp Circuits And Systems, 2005, vol. 3, pp. 2915-2918.

[33] P. Mali and R. Lal, "The DNASET: a novel device for single-molecule DNA sequencing," IEEE Trans. Electron Devices, vol. 51, no. 12, pp. 2004-2012, 2004.

[34] K. Y. Park, K. M. Park, Y. S. Cho, H. Kim, Y. Bae, and S. Choi, "Fabrication and characteristics of bioFET Albumin sensor using new self-assembled monolayer," in Int. Conf. Solid-State Sensors, Actuators and Microsystems, 2005, pp. $1788-1791$.

[35] D. A. Michels, S. Hu, R. M. Schoenherr, M. J. Eggertson, and N. J. Dovichi, "Fully automated two-dimensional capillary electrophoresis for high sensitivity protein analysis," Molecular Cellular Proteomics, vol. 1, pp. 69-74, 2002.

[36] T. Kamei, J. R. Scherer, B. M. Paegel, E. T. Lagally, A. M. Skelley, R. A. Street, and R. A. Mathies, "Microfluidic genetic and amino acid analysis using an integrated a-Si:H detector," in Proc. IEEE Sensors, 2003, pp. 1049-1053.

[37] E. T. Lagally, , J. R. Scherer, and R. G. Blazej et al., "Integrated portable genetic analysis microsystem for pathogen/infectious disease detection," Analytical Chemistry, vol. 76, no. 11, pp. 3162-3170, 2004. 
[38] T. Kamei, N. M. Toriello, E. T. Lagally, R. G. Blazej, J. R. Scherer, R. A. Street, and R. A. Mathies, "Microfluidic genetic analysis with an integrated a-Si:H detector," J. Biomedical Microdevices, vol. 7, no. 2, pp. 147-152, 2005.

[39] T. Smekal, D. Rhine, D. Weston, and P. Grodzinski, "Design, fabrication and testing of thermal components and their integration into a microfluidic device," in IEEE Intersoc. Conf. Thermal Phenomena, 2002, pp. 1039-1045.

[40] E. T. Lagally, I. Medintz, and R. A. Mathies, "Single-molecule DNA amplification and analysis in an integrated microfluidic device," Analytical chemistry, vol. 73, pp. 565-570, 2001.

[41] E. T. Lagally and R. A. Mathies, "Monolithic integrated PCR reactor-CE system for DNA amplification and analysis to the single molecule limit," in IEEE-EMBS Microtechnologies Medical Biology, 2002, pp. 437-441.

[42] M. U. Kopp, A. J. de Mello, and A. Manz, "Chemical amplification: continuousflow PCR on a chip," Science Magazine, vol. 280, pp. 1046-1048, 1998.

[43] C. G. Koh, W. Tan, M. Zhao, A. J. Ricco, and Z. H. Fan, "Integrating polymerase chain reaction, valving, and electrophoresis in a plastic device for bacterial detection," Analytical Chemistry, vol. 75, no. 17, pp. 4591-4598, 2003.

[44] V. P. Iordanov et al., "PCR array on chip thermal characterization," in Proc. IEEE sensors, 2003, vol. 2, pp. 1045-1048.

[45] V. P. Iordanov et al., "Sensorized nanoliter reactor chamber for DNA multiplication," in Proc. IEEE sensors, 2004, pp. 229-232.

[46] I. T. Young, V. P. Iordanov, H. R. C. Dietrich, and A. Bossche, "Nanoliter array advances: miniaturized, high-speed PCR sensing and control," in IEEE Int. Symp. Circuits And Systems, 2005, pp. 2919-2922.

[47] L. A. Legendre, J. M. Bienvenue, M. G. Roper, J. P. Ferrance, and J. P. Landers, "A simple, valveless microfluidic sample preparation device for extraction and amplification of DNA from nanoliter-volume samples," Analytical Chemistry, vol. 78 , no. 5 , pp. 1444-1451, 2006.

[48] E. T. Lagally and R. A. Mathies, "Integrated PCR-CE system for DNA analysis to the single molecule limit," in Micro Total Analysis Systems, 2001, pp. 117118.

[49] J. W. Hong, H. Hagiwara, T. Fujii, H. Machida, M. Inoue, M. Seki, and I. Endo, "Separation and collection of a specified DNA fragment by chip-based CE system," in Micro Total Analysis Systems, 2001, pp. 113-114. 
[50] L. Jang, M. S. Saini, M. R. Holl, and D. R. Meldrum, "Purification of DNA sequencing products with a model compound in a high-throughput microfluidic format, the ACAPELLA-5K," in Micro Total Analysis Systems, 2001, pp. 115116.

[51] C. A. Emrich, I. L. Medintz, H. Tian, L. Berti, and R. A. Mathies, "(ultra)²-high throughput genetic analysis using microfabricated capillary array electrophoresis devices," in Micro Total Analysis Systems, 2001, pp. 13-15.

[52] B. M. Paegel, C. A. Emrich, R. G. Blazej, C. J. Elkin, J. R. Scherer, and R. A. Mathies, "Microfabricated capillary array electrophoresis: high-throughput DNA sequencing and polymorphism analysis," in Micro Total Analysis Systems, 2001, pp. 462-464.

[53] D. Branton, "Nanopore transducers: prospects for single molecule electrophoresis," in IEEE Conf. Solid State Sensors, Actuators and Microsystems, 2003, pp. $210-213$.

[54] D. W. Deamer and D. Branton, "Characterization of nucleic acids by nanopore analysis," Accounts Chem Res, vol. 35, no. 10, pp. 817-825, 2002.

[55] S. Purushothaman, C. Toumazou, and J. Georiou, "Towards fast solid state DNA sequencing," in IEEE Int. Symp. Circuits And Systems, 2002, vol. IV, pp. 169-172.

[56] C. Mattocks, "Evaluation report: automated extraction methodologies," Tech. Rep., National Genetics Reference Laboratory (Wessex), Salisbury, UK, Nov. 2004.

[57] M. Viaznikova and T. Mussivand, "Evaluation of a new DNA sampling and extraction method," in A multidisciplinary congress in Cardio-Thoracic healthcare, August 2006, pp. 88-89.

[58] J. M. Graham and J. A. Higgins, Membrane analysis, BIOS Scientific, NY, USA, 1997.

[59] T. Mussivand, Method and apparatus for collecting cells for macromolecular analysis, US 2006/0008903 A1, Jan. 2006.

[60] K. J. Muller, V. L. Sukhorukov, and U. Zimmermann, "Reversible electropermeabilization of mammalian cells by high-intensity, ultra-short pulses of submicrosecond duration," J. Membrane Biol., vol. 184, no. 2, pp. 161-170, 2001.

[61] K. Maswiwat, D. Wachner, and J. Gimsa, "Effects of cell orientation and electric field frequency on the transmembrane potential induced in ellipsoidal cells," Bioelectrochemistry, vol. 74, pp. 130-141, 2008. 
[62] H. Huelsheger, J. Potel, and E. G. Niemann, "Killing of bacteria with electric pulses of high electric field strength," Radiat. Environ. Biophys., vol. 20, no. 1, pp. 53-65, 1981.

[63] C. Rosa, P. A. Tilley, J. D. Fox, and K. V. Kaler, "Microfluidic device for dielectrophoresis manipulation and electrodisruption of respiratory pathogen bordetella pertussis," IEEE Trans. on Biomedical Engineering, vol. 55, no. 10, pp. 2426-2432, 2008.

[64] F. Han et al., "Fast electrical lysis of cells for capillary electrophoresis," Analytical Chemistry, vol. 75, no. 15, pp. 3688-3696, 2003.

[65] H. Wang, A. K. Bhunia, and C. Lu, "A microfluidic flow-through device for high throughput electrical lysis of bacterial cells basaed on continuous DC voltage," Biosensors and Bioelectronics, vol. 22, pp. 582-588, 2006.

[66] D. W. Lee and Y. Cho, "A continuous electrical cell lysis device using a low DC voltage for a cell transport and rupture," Sensors and Actuators, vol. B 124, pp. 84-89, 2007.

[67] A. J. Sale and W. A. Hamilton, "Effects of high electric fields on microorganisms I: killing of bacteria and yeasts," Biochimica et Biophysica Acta, vol. 148, no. 3, pp. 781-788, 1967.

[68] A. J. Sale and W. A. Hamilton, "Effects of high electric fields on microorganisms III: lysis of erythrocytes and protoplasts," Biochimica et Biophysica Acta, vol. 163, no. 1, pp. 247-267, 1968.

[69] J. G. Korvink and O. Paul, MEMS: A practical guide to design, analysis, and applications, Springer, NY, USA, 2006.

[70] S. D. Senturia, Microsystem design, Springer, NY, USA, 2004.

[71] C. T. Leondes, MEMS/NEMS handbook: techniques and applications, Springer, NY, USA, 2005.

[72] M. R. Knapp, A. Kopf-Sill, R. Dubrow, A. Chow, R. Chien, C. Chow, and J. W. Parce, "Commercialized and emerging lab-on-a-chip applications," in Micro Total Analysis Systems, 2001, pp. 7-9.

[73] M. Roukes, "Nanomechanical systems: application in biotechnology," in Micro Total Analysis Systems, 2001, pp. 1-3.

[74] K. B. Lee and L. Lin, "Surface micromachined glass and polysilicon microchannels using MUMPs," in Proc. IEEE Int. Conf. MEMS, 2003, pp. 578-581.

[75] A. S. Gerstein, Molecular biology problem solver: a laboratory guide, Wiley-Liss, NY, USA, 2001. 
[76] M. Matsunaga, T. Fukuba, T. Yamamoto, and T. Fujii, "Microfabricated devices for DNA extraction toward realization of deep-sea in situ gene analysis," in MTS/IEEE Techno-Ocean, 2004, vol. 1, pp. 89-94.

[77] H. Lu, R. J. Jackman, S. Gaudet, M. Cardone, M. A. Schmidt, and K. F. Jensen, "Microfluidic devices for cell lysis and isolation of organelles," in Micro Total Analysis Systems, 2001, pp. 297-298.

[78] R. B. Brown and J. Audet, "Current techniques for single-cell lysis," J. R. Soc. Interface, vol. 5, pp. S131S138, 2008.

[79] H. Lai, P. A. Quinto-Su, C. E. Sims, M. Bachman, G. P. Li, V. Venugopalan, and N. L. Allbritton, "Characterization and use of laser-based lysis for cell analysis on-chip," J. R. Soc. Interface, vol. 5, pp. S113-S121, 2008.

[80] M. D. Dhawan, F. Wise, and A. J. Baeumner, "Development of a laser-induced cell lysis system," Analytical Bioanalytical Chemistry, pp. 421-426, 2002.

[81] R. W. Doebler, B. Erwin, A. Hickerson, B. Irvine, D. Woyski, A. Nadim, and J. D. Sterling, "Continuous-flow, rapid lysis devices for biodefense nucleic acid diagnostic systems," J. Association for Laboratory Automation, vol. 14, no. 3, pp. 119-125, 2009.

[82] S. W. Lee and Y. C. Tai, "A micro cell lysis device," Sensors and Actuators, vol. 73, pp. 74-79, 1999.

[83] D. W. Lee and Y. Cho, "A continuous cell lysis device using focused high electric field and self-generated electroosmotic flow," in IEEE Int. Conf. MEMS, 2006, pp. $426-429$.

[84] H. Lu, M. A. Schmidt, and K. F. Jensen, "A microfluidic electroporation device for cell lysis," The Royel Society of Chemistry, vol. 5, pp. 23-29, 2005.

[85] H. Wang and C. Lu, "High-throughput and real-time study of single cell electroporation using microfluidic: effects of medium osmolarity," Biotechnology and Bioengineering, pp. 1116-1125, 2006.

[86] K. Lu, A. M. Wo, Y. Lo, K. Chen, C. Lin, and C. Yang, "Three dimentional electrode array for cell lysis via electroporation," Elsevier Biosensors and Bioelectronics, vol. 28, no. 1, pp. 24-33, 2006.

[87] Tai et al., Cell Lysis Device, US006287831B1, Sept. 2001.

[88] P. J. Marc, C. E. Sims, M. Bachman, G. P. Lia, and N. L. Allbritton, "Fast-lysis cell traps for chemical cytometry," Lab on a Chip, vol. 8, pp. 710716, 2008. 
[89] S. Chen and S. J. Lillard, "Continuous cell introduction for the analysis of individual cells by capillary electrophores," Analytical Chemistry, vol. 73, no. 1, pp. 111-118, 2001.

[90] K. Zhua, H. Jin, Y. Maa, Z. Renb, C. Xiao, Z. Hea, F. Zhang, Q. Zhua, and B. Wang, "A continuous thermal lysis procedure for the large-scale preparation of plasmid DNA," Journal of Biotechnology, vol. 118, pp. 257264, 2005.

[91] M. A. McClain, C. T. Culbertson, S. C. Jacobson, and J. M. Ramsey, "Single cell lysis on microfluidic devices," in Micro Total Analysis Systems, 2001, pp. 301-302.

[92] Culburtson et al., Microfluidic systems and methods for transport and ysis of cells and analysis of cell lysate, US006783647B2, Aug. 2004.

[93] S. K. Jha, G. Ra, G. Joo, and Y. Kim, "Electrochemical cell lysis on a miniatured flow-though device," Current Applied Physics, vol. 9, pp. e301-e303, 2009.

[94] N. R. Munce, J. Li, P. R. herman, and L. Lilge, "Microfabricated system for parallel single-cell capillary electrophoresis," Analytical Chemistry, vol. 76, no. 17, pp. 4983-4989, 2004.

[95] U. Schmidt-Erfurth, R. Birngruber, and T. Hasan, "Photodynamic therapy in ocular vascular disease," IEEE J. Selected Topics in Quantum Electronics, vol. 2, no. 4, pp. 988-996, 1996.

[96] J. Black and G. Hastings, Handbook of biomaterial properties, Champman \& Hall, NY, USA, 1998.

[97] J. Gao, X. Yin, and Z. Fang, "Integration of single cell injection, cell lysis, separation and detection of intercellular constituents on a microfluidic chip," Lab Chip, vol. 4, pp. 47-52, 2004.

[98] Y. Huang and B. Rubinsky, "Microfabricated electroporation chip for single cell membrane permeabilization," Sensors and Actuators, vol. 89, pp. 242-249, 2001.

[99] Y. Huang and B. Rubinsky, "Micro-electroporation: improving the efficiency and understanding of electrical permeabilization of cells," Biomedical Microdevices, vol. 2, no. 2, pp. 145-150, 1999.

[100] Y. Huang and B. Rubinsky, "Flow-through micro-electroporation chip for high efficiency single-cell manipulation," Sensors and Actuators, vol. 104, pp. 205212, 2003. 
[101] T. Y. Tsong, "Electroporation of cell membranes," Biophysics J., vol. 60, pp. 297-306, 1991.

[102] E. Neumann, "Electric field-induced structural rearrangements in biomembranes," Studia Biophysic, vol. 130, no. 1-3, pp. 139-143, 1989.

[103] J. C. Weaver, "Electroporation of cells and tissues," IEEE Trans. Plasma Science, vol. 28, no. 1, pp. 24-33, 2000.

[104] D. Moldovan, D. Pinisetty, and R. V. Devireddy, "Molecular dynamics simulation of pore growth in lipid bilayer membranes in the presence of edge-active agents," Applied Physics Letters, vol. 91, no. 20, pp. 204104-1-3, 2007.

[105] R. P. Joshi and K. H. Schoenbach, "Electroporation dynamics in biological cells subjected to ultrafast electrical pulses: a numerical simulation study," Physical Review E, vol. 62, no. 1, pp. 1025-1033, 2000.

[106] E. Neumann, "Membrane electroporation and direct gene transfer," Bioelectrochemistry and Bioenergetics, vol. 28, no. 1-2, pp. 37-43, 1992.

[107] C. Schultheiss, H. J. Bluhm, H. Mayer, and M. Kern, "Development of an industrial electroporation device," in IEEE Power Modulator Symposium, 2002, pp. 563-566.

[108] A. Jenkins, C. P. Chen, S. Spearing, L. A. Monaco, A. Steele, and G. Flores, "Design and modelling of a microfluidic electro-lysis device with controlling plates," in Int. MEMS Conf., 2006, pp. 620-625.

[109] K. H. Schoenbach, F. E. Peterkin, R. W. Alden, and S. J. Beebe, "The effect of pulsed electric fields on biological cells: experiments and applications," IEEE Trans. Plasma Science, vol. 25, no. 2, pp. 284-292, 1997.

[110] K. H. Schoenbach, R. Nuccitelli, and S. J. Beebe, "ZAP extreme voltage could be a surprisingly delicate tool in the fight against cancer," IEEE Spectrum, vol. 43, no. 8(NA), pp. 22-26, August 2006.

[111] P. J. Marc, C. E. Sims, M. Bachman, G. P. Lia, and N. L. Allbritton, "A microfluidic device for physical trapping and electrical lysis of bacterial cells," Applied Physics Letters, vol. 92, pp. 214103-1-3, 2008.

[112] S. B. Dev, D. P. Rabussay, G. Widera, and G. A. Hofmann, "Medical applications of electroporation," IEEE Trans Plasma Science, vol. 28, no. 1, pp. 206-223, 2000.

[113] B. Gross, S. Kandlikar, B. Oakley, D. Hanna, A. Rusek, and G. Stryker, “An examination of the effect of an AC pulsed electric field on cell mortality in SWLA-2 hybridomas," in IEEE Eng. Medical Biological Soc., 2004, pp. 26352638. 
[114] K. H. Schoenbach, A. Abou-Ghazala, T. Vithoulkas, R. W. Alden, R. Turner, and S. Beebe, "The effect of pulsed efields on biological cells," in IEEE Int. Pulsed Power Conf., 1997, vol. 1, pp. 73-78.

[115] K. H. Schoenbach, R. P. Joshi, R. H. Stark, F. C. Dobbs, and S. J. Beebe, "Bacterial decontamination of liquids with pulsed electric fields," IEEE Trans. Dielectrics and Elect. Insulation, pp. 637-645, 2000.

[116] C. Grosse and H. P. Schwan, "Cellular membrane potentials induced by alternating fields," Biophys. J., vol. 63, no. 6, pp. 1632-1642, 1992.

[117] P. T. Vernier, A. Li, L. Marcu, C. M. Craft, and M. A. Gunderson, "Ultrashort pulsed electric fields induce membrane phospholipid translocation and caspase activation: differential sensitivities of Jarkut $\mathrm{T}$ Lymphoblasts and rat Glioma C6 cells," IEEE Trans. Dielectrics and Electrical Insulation, vol. 10, no. 5, pp. 795-809, 2003.

[118] H. Fujimoto, K. Kato, and H. Iwata, "Electroporation microarrray for parallel transfer of small interfering RNA into mamalan cells," Anal. Bioanal. Chem., vol. 392, pp. 1309-1316, 2008.

[119] P. Vulto, G. Dame, U. Maier, S. Makohliso, S Podszun, Peter Zahna, and G. A. Urban, "A microfluidic approach for high efficiency extraction of low molecular weight RNA," Royal Society of Chemistry, vol. 10, pp. 610616, 2010.

[120] A. Chiabrera, C. Nicolini, and H.P. Schwan, Interactions between electromagnetic fields and cells, Plenum Press, NY, USA, 1985.

[121] J. W. Essex, M. M. Hann, and W. G. Richards, "Molecular dynamics simulation of a hydrated phospholipid bilayer," Phil. Trans. R. Soc. Lond., vol. 344B, pp. 239-260, 1994.

[122] M. Tarek, "Membrane electroporation: a molecular dynamics simulation," Biophysical J., vol. 88, pp. 4045-4053, 2005.

[123] S. W. I. Siu and S. A. Bockmann, "Electric field effects on membranes: Gramicidin A as a test ground," J. Structural Biology, vol. 157, pp. 545-556, 2007.

[124] R. M. Campbell, B. H. Crichton, R. A. Fouracre, and M. D. Judd, "Simulated pulse response of intracellular structures in biological cells exposed to highintensity sub-microsecond pulsed electric field," in Conf Electrical Insulation and Dielectric Phenomena, 2005, pp. 377-380.

[125] S. Koda, Y. Inoue, and H. Iwata, "Gene transfection into adherent cells using electroporation on a dendrimer-modified gold electrode," Langmuir, vol. 24, no. 23, pp. 13525-13531, 2008. 
[126] Y. Sun, P. T. Vernier, M. Behrend, L. Marcu, and M. A. Gundersen, "Electrode microchamber for noninvasive perturbation of mammalian cells with nanosecond pulsed electric fields," IEEE Trans. on Nanobioscience, vol. 4, no. 5, pp. 277-283, 2005.

[127] A. S. Bhagat, S. Dasgupta, R. K. Banerjee, and I. Papautsky, "Effects of microchannel cross-section and applied electric field on electroosmotic mobility," in Conf Solid-State Sensors, Actuators and Microsystems, 2007, pp. 1853-1856.

[128] P. Linderholm, U. Seger, and P. Renaud, "Analytical expression for electrical field between two facing strip electrodes in microchannel," Electronic Letters, vol. 42 , no. 3 , pp. 145-146, 2006.

[129] A. N. Chatterjee and N. R. Aluru, "Combined circuit/device modeling and simulation of integrated microfluidic system," IEEE J MEMS, vol. 14, no. 1, pp. 81-95, 2005.

[130] F. T. Ulaby, Fundamentals of applied electromagnetics, Pearson Prentice Hall, NJ, USA, 2004.

[131] J. W. Nilsson and S. A. Riedel, Electric circuits, Prentice-Hall, NJ, USA, 2000.

[132] U. Danielsson, "Convective heat transfer measured directly with a heat flux sensor," American Physiological Society, vol. 68, no. 3, pp. 12751281, 1990.

[133] J. P. Holman, Heat transfer, McGraw-Hill, CA, USA, 1976.

[134] J. H. Hienhard, A heat transfer textbook, Philogiston Press, TX, USA, 2008.

[135] R. Westermeier, Electrophoresis in practice, Wiley-VCH, Weinheim, Germany, 2001.

[136] S. J. Beebe, P. M. Fox, L. J. Rec, K. Somers, R. H. Stark, and K. H. Schoenbach, "Nanosecond pulsed electric field (nsPEF) effects on cells and tissues: apoptosis induction and tumor growth inhibition," IEEE Trans. on Plasma Science, vol. 30, no. 1, pp. 286-292, 2002.

[137] J. Olofsson, M. Levin, A. Stromberg, S. G. Weber, F. Ryttsen, , and O. Orwar, "Generation of focused electric field patterns at dielectric surfaces," Anal. Chem., vol. 77, pp. 4667-4672, 2005.

[138] J. Olofsson, M. Levin, A. Stromberg, S. G. Weber, F. Ryttsen, , and O. Orwar, "Scanning electroporation of selected areas of adherent cell cultures," Anal. Chem., vol. 79, pp. 4410-4418, 2007.

[139] K. Park, D. Akin, and R. Bashir, "Electrical capture and lysis of vaccinia virus particles using silicon nano-scale probe array," Biomed Microdevices, vol. 9, pp. 877883, 2007. 